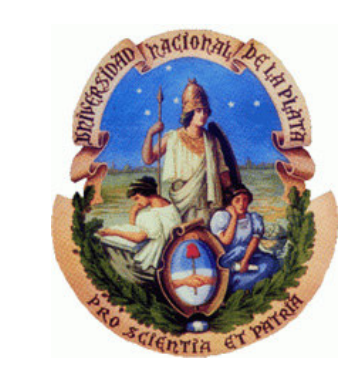

\title{
Control Predictivo del Convertidor Multinivel con Enclavamiento por Diodos y sus Aplicaciones en Media Tensión
}

Tesis doctoral presentada por

\section{Santiago Andrés Verne}

ante la Facultad de Ingeniería de la Universidad Nacional de La Plata para acceder al grado académico de

DOCTOR EN INGENIERIA

Dirección de tesis: Dra. María Inés Valla

La Plata, marzo de 2012 



\section{Resumen}

En esta tesis se realiza el estudio del convertidor multinivel con enclavamiento por diodos. Se presenta un algoritmo generalizado para el control del balance de las tensiones del bus de continua del convertidor que no requiere de circuitos adicionales de balance. También se realiza un análisis de las características de conmutación de la topología y se elaboran reglas que aseguren el funcionamiento seguro de las llaves de potencia. Se desarrolla un algoritmo de control predictivo de estados finitos en el cual se unifica el control de las variables externas del convertidor (corrientes, potencias o variables internas de la carga) con el algoritmo de control de balance. Se consideran las restricciones de conmutación propias de la topología con el propósito de asegurar la operación correcta del convertidor y el sistema que lo circunda. Se analiza el desempeño del convertidor y la técnica desarrollada en aplicaciones de acondicionamiento de potencia, accionamientos eléctricos y generación eólica. Los algoritmos se ensayan mediante simulación en las aplicaciones mencionadas. 



\begin{abstract}
An in depth study of the Diode Clamped Multilevel Converter is performed in this Thesis. The DC bus voltage unbalance problem of the topology is presented and the development of a generalized algorithm for voltage balance control of the DC bus capacitors is addressed which does not need any additional balancing circuits. An analysis is presented concerning the switching constraints of the converter and the synthesis of a switching rule that ensures correct voltage clamping of the power devices. Also, a Finite Control Set Model Predictive Control strategy is developed to merge the voltage balancing algorithm with the control of external variables (currents, power or intrinsic load variables). This algorithm incorporates the switching restrictions of the topology in order to ensure the proper operation of the converter and the control of the external system. The performance of the control algorithm is evaluated by means of computer simulations in applications such as power conditioning, motor drives and wind power conversion.
\end{abstract}



A mis viejos por haberme enseñado sobre el esfuerzo y el trabajo A mi querida Vero y a mis adoraditos Estani y Clarita 



\section{$\underline{\text { Contenidos }}$}

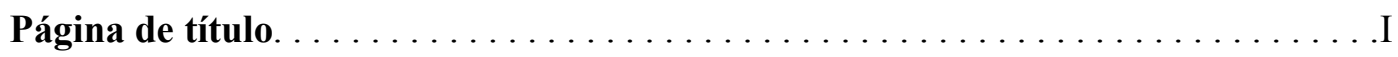

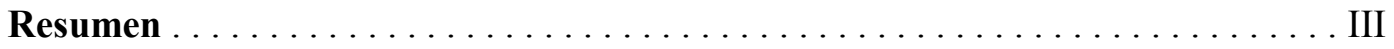

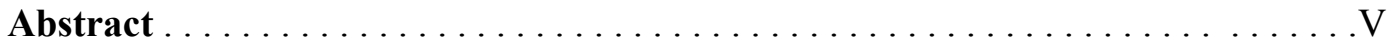

Agradecimientos. ..........................

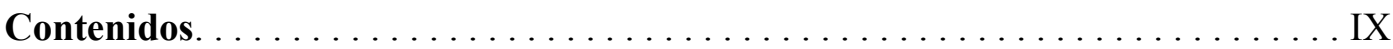

Lista de abreviaturas. . . . . . . . . . . . . . . . . . . . .

\section{Capítulo 1: Introducción}

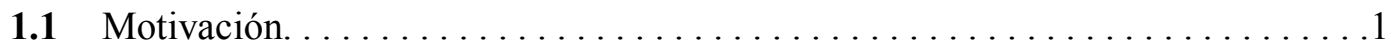

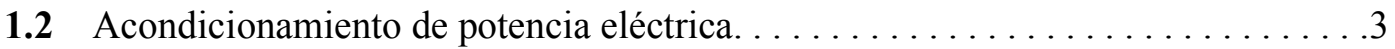

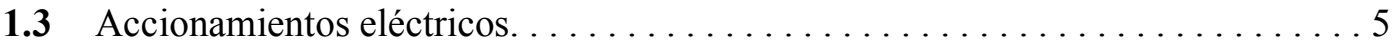

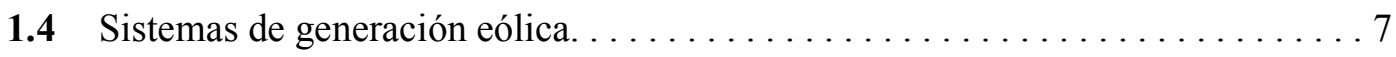

1.5 Convertidores electrónicos como interfaz en media tensión. . . . . . . . . 10

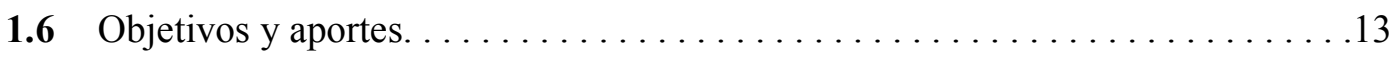

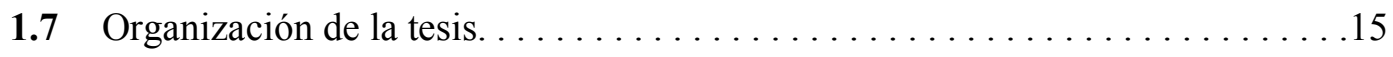

\section{Capítulo 2: Convertidores multinivel}

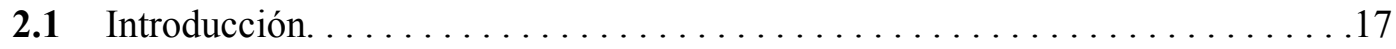

2.2 Clasificación de convertidores de potencia $D C / A C \ldots \ldots \ldots \ldots \ldots \ldots$

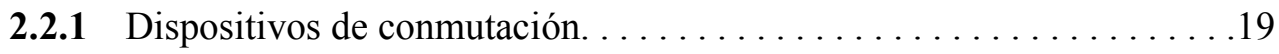

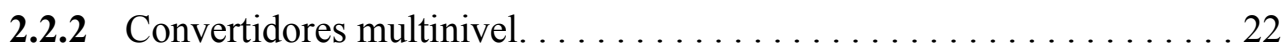

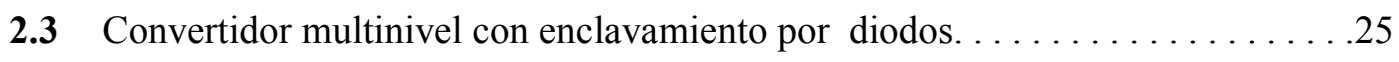

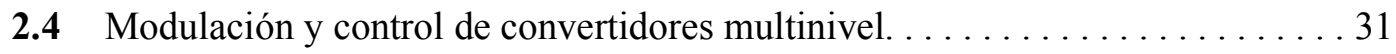

2.4.1 Modulación vectorial en convertidores multinivel. . . . . . . . . . 32

2.4.1.1 Desarrollo del método..................... 34

2.4.1.2 Coordenadas hexagonales. ................ 37 
2.4.1.3 Búsqueda de $\operatorname{los} N T V \ldots \ldots \ldots \ldots \ldots \ldots \ldots \ldots \ldots \ldots$

2.4.1.4 Cálculo de los ciclos de trabajo. ...............43

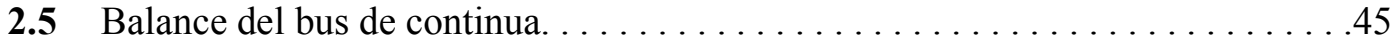

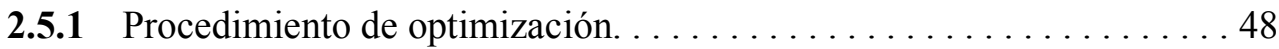

2.5.2 Región de estabilidad de la estrategia de balance. . . . . . . . . . 50

2.6 Aplicación a un compensador de potencia reactiva. . . . . . . . . . . . 52

2.7 Conclusiones. . . . . . . . . . . . . . . . . . . . . . . 58

\section{Capítulo 3: Control Predictivo de Convertidores}

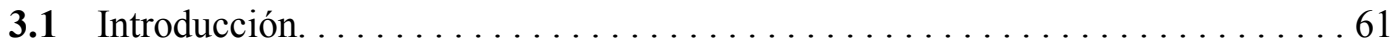

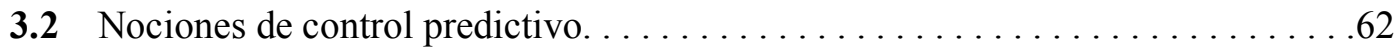

3.3 Control predictivo en convertidores de potencia. . . . . . . . . . . 65

3.4 Control predictivo del convertidor $D C M C \ldots \ldots \ldots \ldots \ldots \ldots \ldots \ldots \ldots \ldots \ldots \ldots$

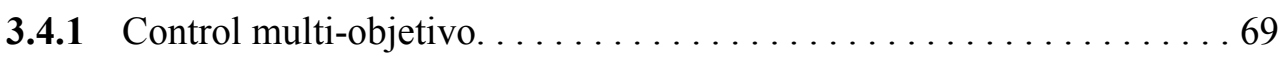

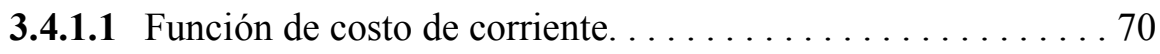

3.4.1.2 Función de costo de balance................ 71

3.4.1.3 Función de costo de reducción de frecuencia de conmutación..73

3.4.2 Diagrama de flujo del algoritmo de control. . . . . . . . . . . . . . 73

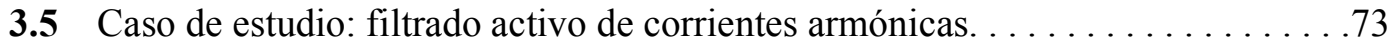

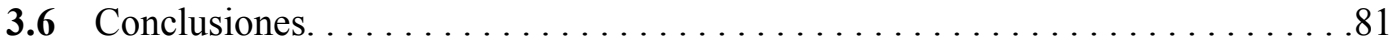

\section{Capítulo 4: El Convertidor DCMC con Frente Activo}

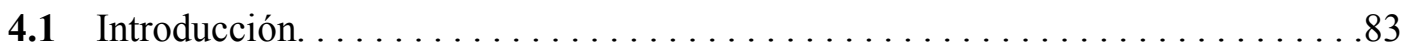

4.2 Conexión $B 2 B$ sobre el $D C M C$. Principio de balance con bus compartido. . . . . 86

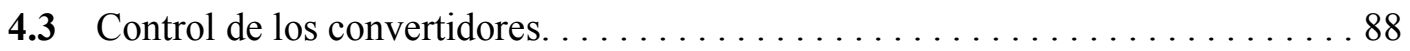

4.3.1 Convertidor de carga: control predictivo de par. . . . . . . . . 92

4.3.2 Convertidor de entrada: control predictivo de potencia. . . . . . . . . .994

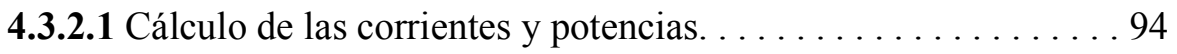

4.3.2.2 Referencia dinámica de potencia activa. . . . . . . . 95

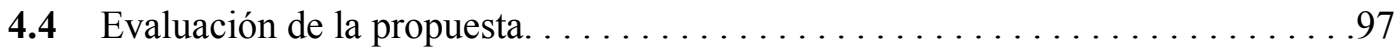

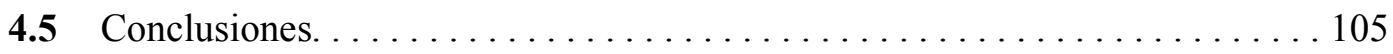




\section{Capítulo 5: Aplicación en Generación Eólica}

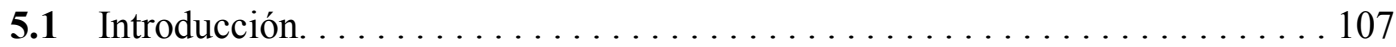

5.2 Topología con generador de imanes permanentes y convertidor

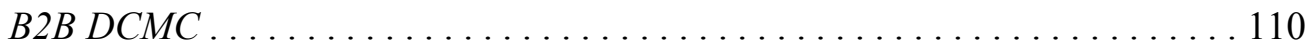

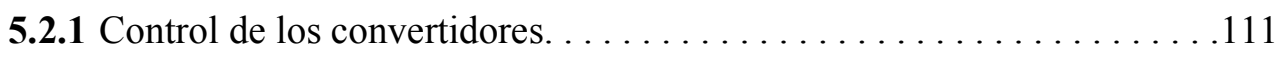

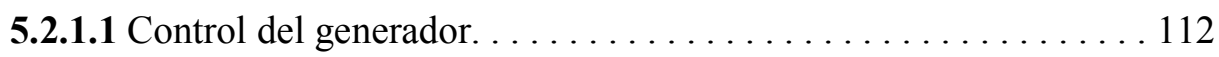

5.2.1.2 Control del convertidor de red. . . . . . . . . . . . . . . . 117

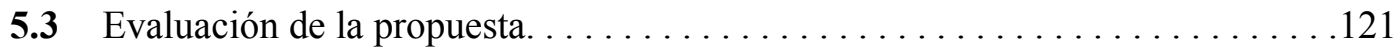

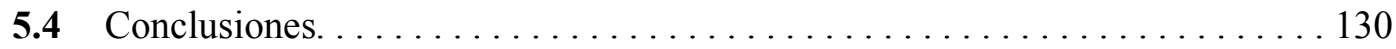

\section{Capitulo 6: Conclusiones}

6.1 Conclusión. ................................. 131

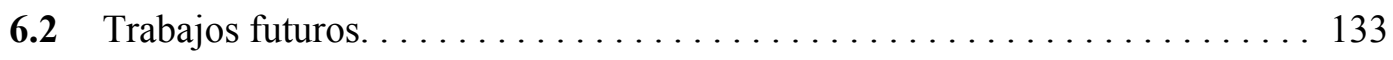

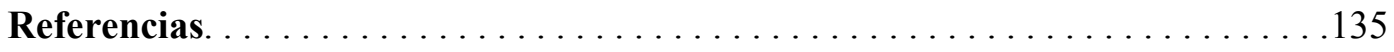





\section{Lista de abreviaturas}

AI: artificial intelligence (inteligencia artificial)

ANN: artificial neural networks (redes neuronales artificiales)

B2B: back-to-back (conexión espalda contra espalda)

BPT: bipolar power transistor (transistor bipolar de potencia)

CC: convertidor de carga

CE: convertidor de entrada

CEP: convertidores electrónicos de potencia

CM: convertidor multinivel

CPP: custom power park (parque dedicado para el acondicionamiento de potencia)

CSC: current source converter (convertidor alimentado con corriente)

CUPS: custom power systems (sistemas dedicados para el acondicionamiento de potencia)

DCMC: diode clamped multilevel converter (convertidor multinivel con enclavamiento por diodos)

DFIG: doubly-fed induction generator (generador de inducción doblemente alimentado)

DSTATCOM: distribution static compensator (compensador estático en nivel de distribución)

DTC: direct torque control (control directo de par)

DVR: dynamic voltage restorer (restituidor dinámico de tensión)

EESG: electrically excited synchronous generator (generador sincrónico con excitación por rotor

EP: electrónica de potencia

FACTS: flexible AC transmission systems (sistemas flexibles de transmisión en corriente alterna)

FAP: filtro activo paralelo

FCC: forced commutated converter (convertidor de conmutación forzada)

FCS-MPC: finite control-set model predictive control (control predictivo basado en modelo con conjunto de control finito)

FOC: field-oriented control (control por orientación de campo)

GC: grid code (código de red) 
LCC: load commutated converter (convertidor conmutado por carga)

MPC: model predictive control (control predictivo basado en modelo)

MRAS: model reference adaptive system (sistema adaptable con modelo de referencia)

MT: media tensión

$\mathbf{N}$ : número de niveles del convertidor multinivel

NPC: neutral point clamp (convertidor con enclavamiento al punto neutro)

NTV: nearest three vectors (tres vectores más cercanos)

PCC: point of common coupling (punto de conexión común)

PFC: power factor corrector (corrector del factor de potencia)

PMSG: permanent magnet synchronous generator (generador sincrónico de imanes permanentes)

PWM: pulse width modulation (modulación por ancho de pulso)

PQ: power quality (calidad de potencia eléctrica)

SSTS: solid state transfer switch (dispositivo de transferencia de estado sólido)

SVC: static VAr compensator (compensador estático de potencia reactiva)

SVM: space vector modulation (modulación del vector espacial)

TCR: thyristor-controlled reactor (reactor controlado a tiristores)

TSC: thyristor-switched capacitor (capacitor conmutado a tiristores)

UPQC: universal power quality conditioner (acondicionador universal de calidad de la potencia eléctrica)

VSC: voltage source converter (convertidor alimentado con tensión)

WECS: wind energy conversion system (sistema de conversión de energía eólica)

WTS: wind turbine system (turbina eólica) 


\section{Capítulo 1}

\section{$\underline{\text { Introducción }}$}

\subsection{Motivación}

La necesidad de optimizar los recursos energéticos y la infraestructura existente impulsa el desarrollo de nuevas soluciones y tecnologías en todos los eslabones de la cadena de procesamiento de la energía eléctrica. Esto tiene lugar en los sistemas de alta potencia e incluso a nivel doméstico. También, la concientización colectiva en materia de preservación del medio ambiente presiona sobre la creación y puesta en marcha de normativas afines a un uso eficiente de los recursos energéticos con el objetivo de reducir los efectos de contaminación.

Es sabido que el uso de la electricidad como medio de distribución, conversión y uso de la energía posee ventajas significativas con respecto a otros mecanismos. Esto se debe a la alta versatilidad con la cual puede convertirse la energía eléctrica en otras formas de energía útiles. Razón de ello es que dicha rama de la tecnología posee una amplia variedad de herramientas y dispositivos capaces de actuar, intermediar y convertir el flujo de potencia. Con el paso del tiempo, esto ha derivado en complejas disciplinas en torno al diseño, construcción, mantenimiento y expansión de las redes y las máquinas.

Desde los orígenes de la era eléctrica, los componentes de las redes y los accionamientos se han basado en principios electromecánicos. Estos elementos han permitido sortear problemas típicos de redes, aumentando la extensión y la calidad de los servicios. En aquellos tiempos el concepto de "calidad del servicio eléctrico" se centraba casi exclusivamente en la continuidad del mismo. Sin embargo, en la actualidad, también se incorporan aspectos relacionados con parámetros específicos, 
como la distorsión de las formas de onda de tensión y corriente y la fluctuación de amplitud, entre otros [1].

Si bien en gran medida los componentes de las redes eléctricas siguen siendo mayormente electromecánicos, el desarrollo y evolución de la electrónica de potencia $(E P)$ ha permitido ampliar el universo de soluciones en esta disciplina. Las nuevas ideas en conjunto con el desarrollo de dispositivos de potencia de última generación no se hacen esperar por parte de los creativos para encontrar nuevas e interesantes aplicaciones de innovación u optimización de los procesos productivos. Además, en algunos casos esta impronta se ve reforzada gracias a la desregulación del mercado eléctrico, donde los operadores de los distintos componentes del sistema exprimen al máximo la capacidad operativa de los equipos e instalaciones a fin de aumentar las utilidades [2].

Es así que la EP viene ganando participación sobre las distintas etapas de la cadena de procesamiento de la energía eléctrica como ser la conversión, transmisión, distribución y el acondicionamiento de potencia. En este sentido, los niveles de tensión y potencia en los cuales participan los convertidores electrónicos se ven limitados básicamente por los dispositivos de conmutación. Estos límites vienen impuestos por la física de funcionamiento y los materiales semiconductores que los componen. En la actualidad puede decirse que la tecnología del silicio es única para todos los dispositivos de conmutación [3]. Sin embargo, existen grandes expectativas alrededor de nuevos materiales semiconductores como el carburo de silicio o de película delgada de diamante para la construcción de las nuevas llaves de potencia [4][5].

Todo esto representa un campo fértil de investigación en torno al estudio del control de los sistemas involucrados y sus componentes, como así también de los convertidores, sus topologías y métodos de control. Particularmente, con respecto a estos últimos es claro que los convertidores electrónicos de potencia $(C E P)$ en conjunto con las veloces plataformas digitales de cómputo disponibles en la actualidad, hacen posible la implementación de sofisticadas estrategias de control y supervisión, las cuales pueden ser ejecutadas con altísima velocidad. 
Los convertidores multinivel constituyen alternativas prometedoras para el procesamiento de potencia en niveles de media tensión $(M T)$. El estudio de dichas soluciones involucra el estudio de las topologías existentes y la búsqueda de nuevos convertidores y métodos de control a fin de asegurar un correcto desempeño con alto nivel de confiabilidad.

\subsection{Acondicionamiento de potencia eléctrica}

Con referencia a los $C E P$ como parte de las redes eléctricas, pueden ubicarse dos tipos de sistemas dependiendo de los niveles de tensión. En el nivel de media tensión, la $E P$ ha incursionado en las problemáticas de calidad de potencia eléctrica ( $P Q$ : power quality) en lo que se conoce como sistemas de acondicionamiento dedicados o CUPS (custom power systems) [1][2]. Esta disciplina abarca el tratamiento de problemáticas puntuales (sobre consumidores particulares o sobre regiones acotadas de la red de suministro), en tanto que a nivel de transmisión, el grupo de soluciones aborda problemas de optimización de la infraestructura eléctrica y el aumento de la flexibilidad de operación de las redes. Dichos dispositivos se denominan sistemas flexibles de transmisión en corriente alterna (FACTS: flexible AC transmission systems). Con mayor precisión, la denominación custom power system se refiere a todo aquel dispositivo instalado para incrementar las prestaciones y/o mejorar la calidad de servicio de uno o más usuarios en el rango de media tensión [1]. En general pueden clasificarse en dispositivos de reconfiguración y dispositivos de compensación. Dentro de los dispositivos de compensación se encuentran aquellos basados en la variación de la impedancia vista desde la línea. Entre estos se encuentran los integrantes de la familia de compensadores estáticos tradicionales SVC (static var compensators), cuyo principio de funcionamiento consiste en la conexión de una reactancia capacitiva en forma total (TSC: thyristor switched capacitor) o gradual por control del ángulo de disparo (TCR: thyristor controlled reactor) para entregar potencia reactiva a la red. Estos dispositivos se basan en tiristores y poseen amplia trayectoria como actuadores en sistemas eléctricos. Sin embargo, los nuevos dispositivos basados en tecnología de conmutación forzada como IGBT y IGCT han permitido incrementar la versatilidad de control con alta velocidad de conmutación, extendiendo las posibilidades para combatir perturbaciones rápidas y ampliando el conjunto de herramientas para su mitigación [6]. 
Dependiendo de la topología de conexionado, este conjunto de dispositivos puede abordar diferentes aspectos de la calidad de la potencia eléctrica. La siguiente es una clasificación de los mismos según el tipo de conexión a la red, y sus potenciales aplicaciones:

1) Conexión en derivación: Compensador estático (DSTATCOM: distribution static compensator). Compensación de reactivo, regulación de tensión, mitigación de flicker, filtrado de armónicas, balance de carga. Este dispositivo puede o no incluir un medio de almacenamiento de energía para la cobertura de fallas de corta duración en la red)

2) Conexión en serie: Compensador de tensión (DVR: dynamic voltage restorer). Puede regular la tensión de salida. Es útil para la estabilización de la tensión de alimentación de cargas o instalaciones críticas como hospitales y procesos productivos altamente sensibles (frecuentemente posee almacenamiento de energía para la cobertura de fallas de corta duración en la red)

3) Conexión combinada: Acondicionador universal de calidad de potencia eléctrica (UPQC: universal power quality conditioner). Es el más versátil de todos los dispositivos (y el de mayor costo), ya que reúne las ventajas de los dos anteriores. Consiste en la conexión espalda contra espalda (B2B: back to back) de dos convertidores, uno de los cuales se conecta en serie con la red y el otro en paralelo.

En la Fig. 1.1 se muestra cada una de las topologías de conexión mencionadas actuando sobre un punto de conexión compartido por múltiples consumidores $(P C C)$.
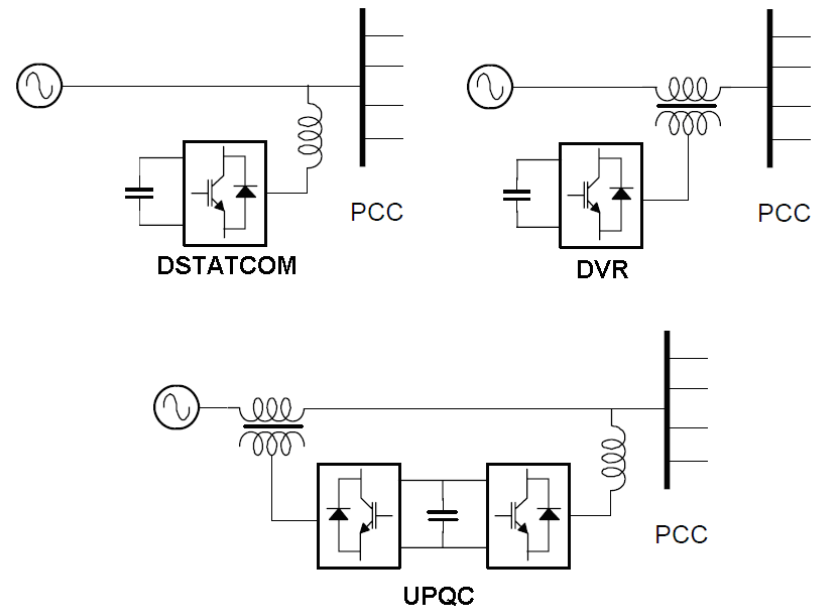

Figura 1.1. Topologías de dispositivos CP, (a) DSTATCOM (b) DVR (c) UPQC 
Es posible integrar las prestaciones de los dos primeros para brindar servicio de alta calidad y confiabilidad a un conjunto de consumidores en un "parque de acondicionamiento de potencia" (CPP: custom power park) [1][7], donde se combina un dispositivo de transferencia de estado sólido (SSTS: solid state transfer switch) que conmuta la alimentación entre dos sub-estaciones distintas (de preferencia y de emergencia), un DSTATCOM para compensar armónicos y desbalance de fases, un DVR para compensar huecos de tensión, y un medio propio de generación eléctrica. Dependiendo de la categoría del parque, también puede incorporarse una fuente de almacenamiento de energía de respaldo para cubrir el lapso de tiempo desde que se produce la falta grave hasta que entra en funcionamiento el generador local.

\subsection{Accionamientos eléctricos}

La aplicación de los $C E P$ en el campo de accionamientos eléctricos de gran porte es un tema de gran importancia en el ámbito industrial. La impulsión de fluidos mediante bombas presurizadoras para transporte en oleoductos y sistemas de purificación y tratamiento de agua, sopladores de gran potencia en la industria del cemento, maquinaria de minería y tracción ferroviaria y naval requieren altos niveles de conversión de potencia. Además dichas instalaciones requieren frecuentemente controlar el caudal, el par o la velocidad de los motores. En este sentido, la utilización de variadores de velocidad electrónicos posee importantes ventajas en cuanto a versatilidad de control y rendimiento energético de los motores, en comparación con las implementaciones a velocidad fija o con regulación mecánica o electromecánica [8][9].

El diseño de un convertidor para accionamiento de motores debe tener en cuenta requerimientos de control y calidad de formas de onda de ambos lados del sistema (alimentación y carga), y a su vez características de implementación y confiabilidad. Para el control de las máquinas existe una variedad de técnicas que dependen del grado de desempeño dinámico requerido por la aplicación. Pueden mencionarse desde el control escalar $\mathrm{V} / \mathrm{Hz}$ con modulación de ancho de pulso hasta técnicas avanzadas de control como el control por orientación de campo (FOC) y control directo de par (DTC).

A nivel académico y experimental también se han estudiado estrategias de control avanzadas basadas en algoritmos de inteligencia artificial $(A I)$. Se encuentran presentes en estos estudios los algoritmos basados en redes neuronales $(A N N)$, lógica difusa (fuzzy 
logic) y control adaptivo (MRAS) entre otros, tanto para control como para identificación de parámetros) [10][11][12].

El diseño de convertidores conmutados para el accionamiento de motores presenta una multiplicidad de factores que merecen consideración, más aún, a medida que aumentan los niveles de potencia y tensión de operación. Algunos de estos aspectos se listan a continuación.

(1) Capacidad de transferencia bidireccional de potencia o de frenado electromagnético.

(2) Contaminación armónica y/o consumo de potencia reactiva sobre el lado de la red de alimentación.

(3) Interacción de la forma de onda de tensión del convertidor con los arrollamientos de la máquina y el circuito magnético. Efectos de los armónicos de tensión de alta frecuencia (reflexiones, sobretensión en bornes, tensiones de modo común, fallas en rodamientos [13][14]) y de corriente de baja frecuencia: degradación de las características de chapa debido a calentamiento excesivo del circuito magnético.

(4) Interferencia electromagnética $(E M I)$

(5) Excitación indeseable del conjunto mecánico debido al rizado del par electromagnético originado en la distorsión de corriente en bobinados del motor.

(6) Necesidad de transformador reductor para accionamientos de gran potencia con conexión a la red de distribución de $M T$.

Los siguientes aspectos de diseño del accionamiento pueden repercutir sobre uno o más de los puntos anteriores:

- Elección de la topología del convertidor

- Utilización de elementos de hardware adicionales

- Algoritmo de control del accionamiento y/o estrategia de modulación del convertidor

Como ejemplo de ello, pueden mencionarse algunas alternativas para abordar los puntos mencionados: 
Para (1): Convertidores bidireccionales (indirectos $(B 2 B)$ o directos (matriciales)), puentes controlados en antiparalelo o hardware adicional de frenado disipativo.

Para (2): Rectificadores con frente activo, rectificadores multipulso, prereguladores elevadores PFC (power factor corrector).

Para (3): Filtros $L C$ de salida, esquemas de modulación con reducción/eliminación de armónicos de baja frecuencia. Convertidores con múltiples niveles.

Para (4): Filtros de salida, cables de conexión especiales, convertidores con múltiples niveles.

Para (5): Algoritmos de control orientados a la máquina, convertidores con múltiples niveles.

Para (6): Convertidores con múltiples niveles

\subsection{Sistemas de conversión eólica}

Otro campo de aplicación de los $C E P$ se relaciona con la producción y el procesamiento de energía a partir de fuentes renovables. Esta actividad involucra la optimización del recurso energético, principalmente solar y eólico, y también en lo que respecta a la interacción del sistema con la red eléctrica en términos de la calidad de potencia, su régimen de producción y funciones complementarias o auxiliares. En este campo también se presentan conceptos novedosos como la diversificación de las fuentes de energía y lo que se presenta como un nuevo paradigma respecto a la estructura de las redes eléctricas: la generación distribuida. Esta rama entraña complejos desafíos en torno al control de las redes, donde nuevamente los $C E P$ son una pieza clave como actuadores de los sistemas de control [15].

Si bien el aprovechamiento de la energía eólica tiene un origen lejano, el desarrollo sistemático de esta tecnología, considerando su potencial como un recurso explotable a gran escala se remonta a sólo 30 años atrás, a partir de la popular turbina Riisager de $22 \mathrm{~kW}$. Desde entonces, el interés ha ido en aumento principalmente gracias a las proyecciones de escasez de combustibles no renovables y el incremento de precios. Por otra parte, debido a que el aumento de tamaño de las turbinas redunda en menor costo 
por unidad de energía generada, la capacidad de generación ha ido creciendo aceleradamente a lo largo de las tres últimas décadas. Esto se muestra en la Fig. 1.2, donde se puede apreciar el aumento de tamaño de las estructuras y la potencia de las máquinas. Actualmente el estándar de la industria son las máquinas de 1 a $2 \mathrm{MW}$ de potencia, llegando a 6-7,5MW para modelos más modernos (Enercon E-126).

En general, en las turbinas modernas la operación a velocidad variable es ampliamente aceptada a nivel industrial gracias a su mayor producción energética anual con respecto a sus pares de velocidad fija. Existen varias combinaciones de tipos de generadores y convertidores de potencia que se encuentran en la bibliografía y a nivel de producción [16][17]. Con respecto a las implementaciones, la máquina de inducción doblemente alimentada (DFIG: doubly fed induction generator) es la más popular en este tipo de sistemas, proveyendo un rango de velocidad aceptable para la maximización de la producción anual de energía de $+-30 \%$. Dicho porcentaje coincide con la relación entre la potencia del convertidor y la potencia de la máquina, limitando su capacidad operativa ante el requerimiento de servicios auxiliares para el operador de la red.

Por otra parte, la topología que utiliza generador sincrónico en conjunto con un convertidor $B 2 B$ en el camino de potencia ha ganado popularidad gracias a que posee plena capacidad operativa $(100 \%$ de la corriente nominal) ante exigencias de cumplimiento de grid codes (GC) [18][19]. Más aún, la atención se particulariza en el generador sincrónico de imanes permanentes (PMSG: permanent magnet synchronous generator) ya que gracias a la disminución del precio de los modernos imanes de neodimio-hierro-boro dicha alternativa se torna económicamente viable [17][20][21]. Este tipo de máquina posee excelentes características pues combina bajo mantenimiento (no posee anillos rozantes), alta relación potencia-peso y permite reducir y hasta omitir la caja reductora, aumentando el rendimiento y la robustez mecánica del conjunto [22]. Luego, la topología de convertidores $B 2 B$ en conjunto con el PMSG combina bajo mantenimiento de las partes mecánicas con alta capacidad para ejecutar servicios auxiliares. 


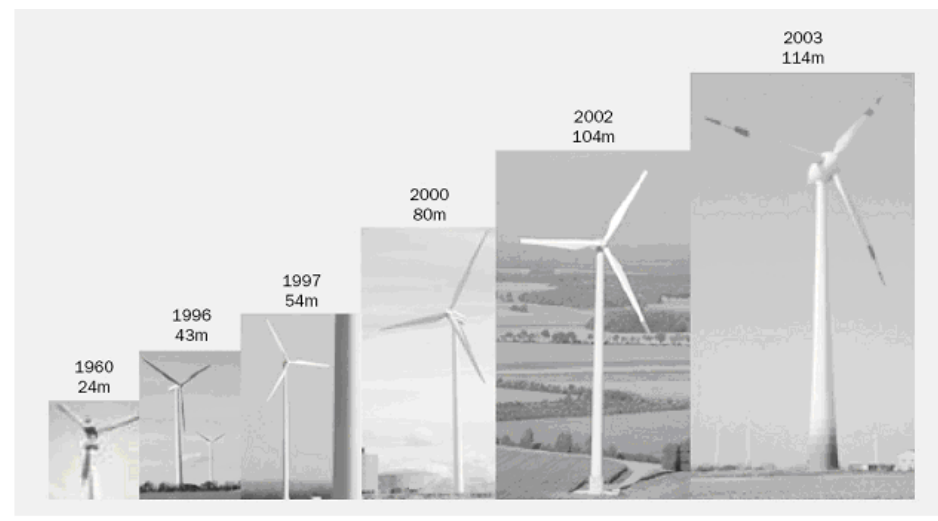

(a)

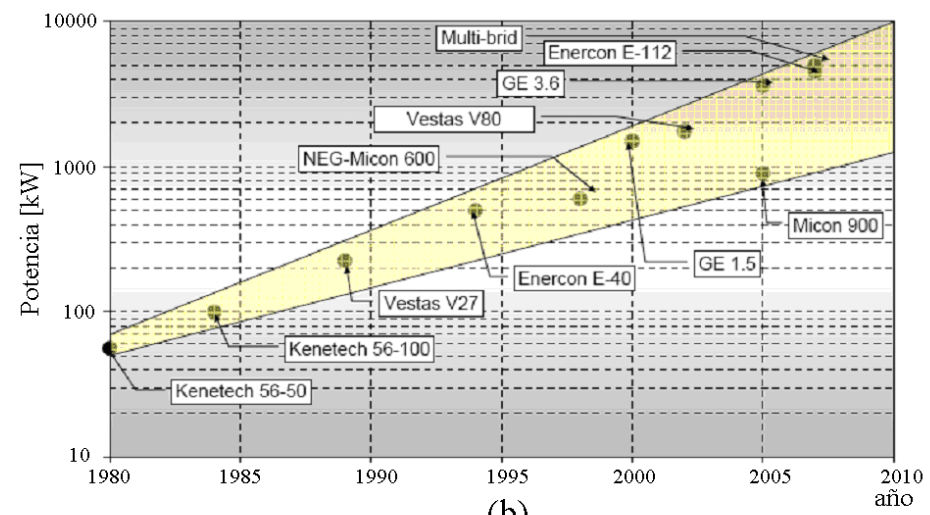

(b)

Figura 1.2. Evolución de las turbinas eólicas, (a)Longitud de pala, (b)Potencia de salida

En el rango de los megavatios, la utilización de equipamiento de media tensión en la etapas de generación y conversión de frecuencia reviste gran interés como alternativa a la conexión en paralelo de convertidores de baja tensión con transformador elevador. En particular, la configuración $B 2 B$, conjuntamente con el $P M S G$ proporciona mayor eficiencia en comparación con la alternativa de rectificador con convertidor $D C / D C$ y también posee mayor flexibilidad de control del lado del generador. Por otra parte, la configuración $B 2 B$ permite ejecutar los $G C$ con $100 \%$ de la corriente nominal de la turbina, en comparación con el $30 \%$ de su competidor basado en el DFIG. Esto constituye una característica de importancia visto la expectativa de creciente penetración de este tipo de sistemas en las redes interconectadas. 


\subsection{Convertidores electrónicos como interfaz en MT}

Los $C E P$ constituyen el corazón operativo de cada una de las aplicaciones mencionadas. En todos los casos la conexión directa (sin transformador de acoplamiento) de los convertidores a la red o a un dispositivo de media tensión es conveniente. En este punto los convertidores multinivel son una alternativa interesante pues extienden los límites intrínsecos de los dispositivos en tanto que mejoran las características de distorsión de las formas de onda sintetizadas para una dada frecuencia de conmutación. En [23][24][25] se presenta una revisión general de topologías, métodos de control y aplicaciones de los convertidores multinivel. Un aspecto clave de la utilización de convertidores multinivel es el control de las tensiones de los capacitores de almacenamiento. Esto constituye una restricción dependiente de la aplicación requiriendo en mayor o menor medida un esfuerzo de elaboración de los algoritmos de control, o bien incorporación de dispositivos complementarios.

En los párrafos siguientes se mencionan las publicaciones más significativas en torno a los convertidores multinivel con relación a las aplicaciones de interés en esta tesis.

\section{Acondicionamiento de potencia eléctrica}

Con referencia al acondicionamiento de potencia eléctrica, varias topologías de convertidores multinivel han sido evaluadas, en general con un número reducido de niveles y en particular cuando se trata del convertidor multinivel con diodos de enclavamiento (DCMC: diode clamped multilevel converter) y de capacitores flotantes [26][27][28][29][30][31]. También se encuentran trabajos con convertidores de más niveles, pero con hardware adicional para el control de balance de las tensiones de los capacitores del bus de continua [32]. En [33][34][35] se analizan algoritmos de control utilizando estados redundantes del convertidor. En [36] se realiza un análisis comparativo para aplicaciones de filtro activo entre el convertidor con enclavamiento al punto neutro $(N P C)$ y el de celdas en cascada. Con respecto a la implementación de los controladores de corriente se observa una variedad de técnicas basadas en moduladores subarmónicos con portadoras desplazadas, en conjunto con control de corriente en cascada y técnicas no lineales [29][37][38]. Un resumen de métodos para la detección de armónicas puede encontrarse en [39] y [40]. 


\section{Accionamientos eléctricos}

La conexión $B 2 B$ de convertidores es una topología de gran interés para accionamientos. Además, la operación directa en $M T$ permite omitir el complicado transformador con devanados en zig-zag a la entrada del sistema. Esto posee ventajas adicionales como el incremento del rendimiento global, relajación de los requerimientos de refrigeración y reducción de pérdidas por efecto Joule en bobinados de la máquina. Por lo tanto, la eliminación del transformador conlleva una reducción significativa de espacio y peso del conjunto ya que representa el 30-50\% del volumen y 50-70\% del peso [41][42] (ver Fig. 1.3). También, en algunas aplicaciones donde la máquina se sitúa lejos del alimentador, el costo de cableado se reduce gracias a la disminución de la sección del cable [43].

Con respecto al control de los $C M$ en estas aplicaciones, la técnica de control predictivo ha sido aplicada debido a la facilidad con la que pueden integrarse al algoritmo las características propias de las topologías [38][44]. En particular respecto a los accionamientos $B 2 B$, se han publicado varios trabajos, donde se analiza el control directo de corriente y de potencia del rectificador de conmutación forzada mediante la teoría de la potencia instantánea [45][46][47][48]. Este mecanismo posee la ventaja de omitir el uso de un modulador y logra gran inmunidad de la tensión del bus de continua ante fluctuaciones de la red o cambios en el flujo de potencia entre la red y la carga. Por otra parte, el control directo de par (DTC: direct torque control) de motores de inducción es una estrategia interesante pues posee buen desempeño en sus características dinámicas prescindiendo de controladores externos. También, esta estrategia se ha extendido a convertidores multinivel y ha sido adaptada al control predictivo [49].

Dentro de las topologías multinivel, el convertidor con enclavamiento por diodos es topológicamente adecuado para la conexión $B 2 B$ [50]. El control del convertidor $B 2 B$ posee varios objetivos, tanto en el lado de la red como en el lado de la máquina, como ser consumo de corriente sinusoidal con bajo contenido de armónicos y alto factor de potencia, y por otro lado el control de las variables internas de la máquina. Debido a que el convertidor multinivel con enclavamiento por diodos impone sus propias 
restricciones de funcionamiento y limitaciones, la estrategia de control debe ser suficientemente elaborada para atender todos los objetivos.

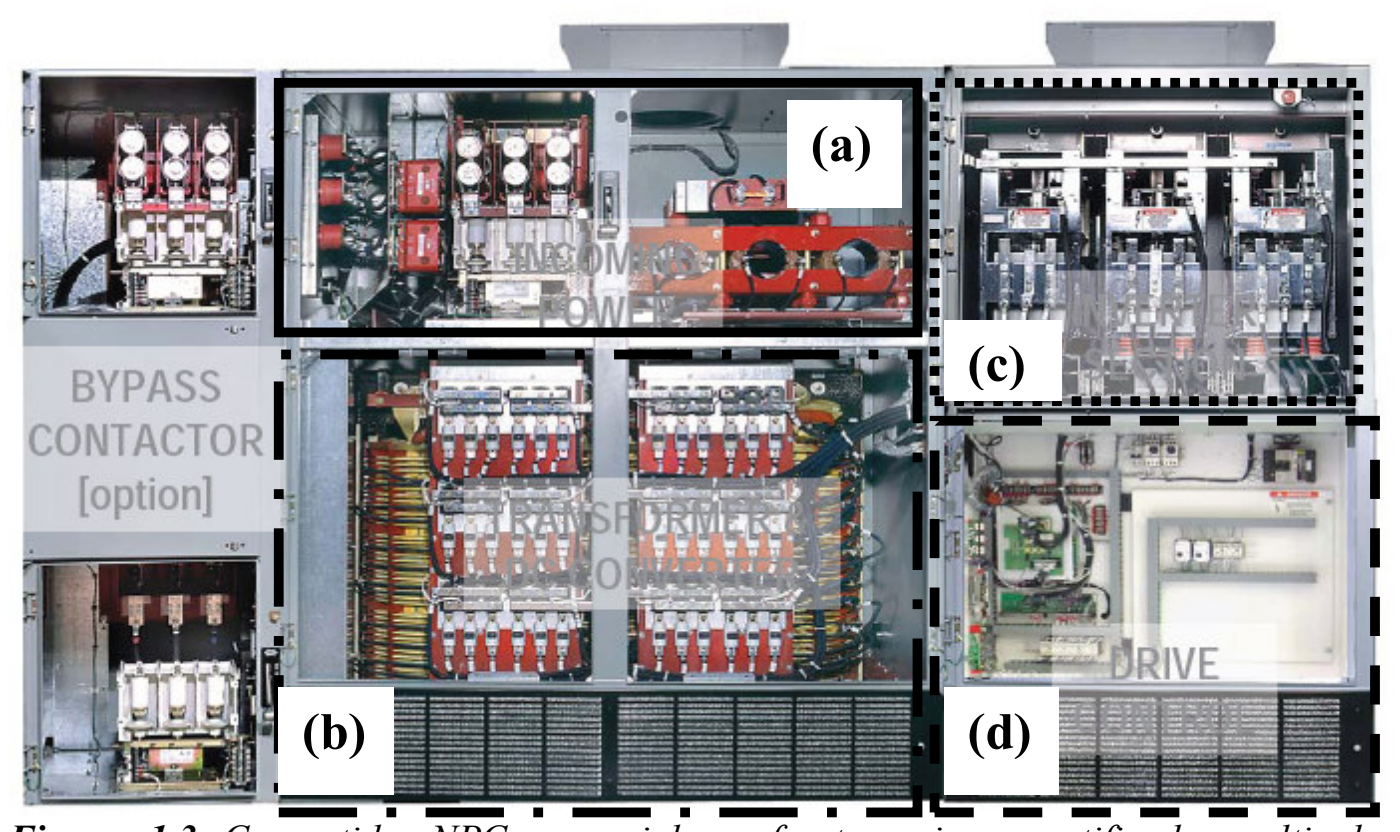

Figura. 1.3. Convertidor NPC comercial con frente pasivo y rectificador multipulso (TMGE Dura-Bilt5i, 1850kVA, 7.5kV), (a) Aparatos de maniobra y protección de la alimentación (b) Transformador y rectificador de 24 pulsos, (c) Inversor NPC, (d) Placa de control

\section{Sistemas de generación eólica}

Con respecto a los WECS, la operación de los convertidores en el rango de media tensión repercute favorablemente en varios aspectos. El primero que puede mencionarse se refiere a la ubicación del transformador de acoplamiento a la red. En este sentido, debe tenerse en cuenta que la barquilla es el lugar natural para la ubicación del transformador. Esto se debe a que cualquier instalación adicional en la parte inferior de la turbina constituye un costo adicional, en especial considerando máquinas instaladas off-shore. Sin embargo, esto se complica debido a restricciones de peso sobre la torre. La segunda razón radica en que para niveles de potencia del orden de los megavatios en niveles de baja tensión, las máquinas deben entregar varios cientos de amperes, causando altas pérdidas por conducción y caída de tensión sobre los cables de conexión. Para superar este problema, dichos conductores se dimensionan con grandes secciones, lo cual impacta negativamente en el costo y en su capacidad de torsión [51]. 
Este contexto, en conjunto con la proyección del aumento en la potencia de las turbinas, sugiere la migración a sistemas de media tensión. En este sentido existe bibliografía en la cual se exploran estas alternativas con topologías de convertidores multinivel [52][53][54]. Desde el punto de vista de la tecnología de generadores sincrónicos multipolares también existe bibliografía al respecto [22][55][56] en tanto que en el ámbito industrial se observa una tendencia en tal sentido [57][58][59].

\subsection{Objetivos y aportes}

En este trabajo se realizó el estudio del convertidor multinivel con enclavamiento por diodos y se caracterizaron sus aspectos funcionales esenciales, como las restricciones de conmutación y el balance de las tensiones del bus de continua. Se desarrolló una esquema de control predictivo de estados finitos para el control de las variables internas y las variables externas, integrando el control en un esquema simple y compacto. El esquema propuesto incorpora explícitamente las restricciones de conmutación de la topología, reduciendo la cantidad de estados a evaluar al número correspondiente a un convertidor de tres niveles. Este resultado arriba a una reducción significativa del costo computacional del algoritmo a medida que el número de niveles aumenta por encima de tres.

Como ejemplos de aplicación, se abordan problemas como la compensación de potencia reactiva y filtrado de armónicas, accionamientos eléctricos en media tensión y en sistemas de generación eólica. El estudio en cada caso involucra la resolución del problema de control de la planta, cuyo actuador es el convertidor, como así también los aspectos de control de las variables internas del mismo, a fin de lograr una operación confiable de todo el conjunto.

Los aportes de este trabajo han sido publicados en congresos y revistas. Los mismos pueden resumirse en los siguientes puntos:

- Se elaboró una estrategia de control de balance de las tensiones del bus de continua para el convertidor multinivel con enclavamiento por diodos. Dicha estrategia tiene carácter general para convertidores con número arbitrario de niveles $[60][61][62][63]$.. 
- Se evaluó la integración del control de variables externas al convertidor con el control del balance de tensiones del bus de continua en un único control predictivo de estados finitos basado en modelo (FCS-MPC).

- Se adaptó el control predictivo diseñado a diferentes aplicaciones como ser:

- Filtro activo de potencia de armónicas [64][65][66].

- Conversor de frecuencia indirecto $(B 2 B)$ basado en la topología DCMC tanto en aplicación de variador de velocidad de un motor de inducción con control predictivo directo de par, como en una aplicación de enlace de corriente continua [67][68][69][70].

- Turbinas de generación eólica en niveles de media tensión [71][72][73]. Algunos aspectos relacionados con esta temática fueron:

1) El estudio de las configuraciones típicas de convertidores y generadores y sus características respecto a la capacidad de ejecutar funciones auxiliares para soporte de la red, en particular la configuración $P M S G$ con esquema de convertidor indirecto en el camino de potencia.

2) La elaboración de un esquema de simulación de una turbina eólica.

3) El diseño de un algoritmo de control predictivo de estados finitos a ser ejecutado por el convertidor capaz de controlar la máquina en su punto óptimo de funcionamiento y a su vez aportar al balance del bus de continua, preservando los condicionamientos de conmutación propios del convertidor.

4) El estudio del problema de la penetración de energías renovables en la red y los requerimientos de interfaz a la misma, condicionamientos de retención de conexión y servicios auxiliares para el operador de la red. En base a ello se diseñó un algoritmo de control de potencia capaz de administrar las potencias activa y reactiva dependiendo del estado de la red (estado normal o en falla).

\subsection{Organización de la tesis}

La tesis se organiza de la siguiente manera: el capítulo 2 presenta un resumen de las topologías multinivel clásicas y se focaliza el análisis en el convertidor multinivel con 
enclavamiento por diodos, su conformación y principio de funcionamiento. También se explican los fundamentos de la técnica de modulación vectorial multinivel y sus problemas de implementación. Se introduce el concepto del sistema de referencia en coordenadas hexagonales que simplifica la selección de los estados de conmutación del convertidor. También se introduce el inconveniente de la topología en cuanto al efecto de desbalance de las tensiones sobre los capacitores del bus de continua y se expone una propuesta para dicho control. Como aplicación de esta estrategia de control se presenta el caso de estudio de un sistema de compensación de potencia reactiva. El capítulo 3 aborda la técnica de control predictivo basado en modelo como estrategia no lineal de control de sistemas y en particular la variante de estados finitos aplicada a convertidores conmutados. Se introduce un modelo funcional del convertidor $D C M C$ teniendo en cuenta las restricciones intrínsecas de la topología, indispensables a fin de preservar la integridad de los dispositivos de conmutación. Se analizan las ventajas del uso de esta estrategia para el control de las corrientes de salida del convertidor y de balance de tensiones del bus $D C$. Se presenta el estudio de un filtro activo de potencia y se exploran sus características. En el capítulo 4 se introducen las ventajas generales de los convertidores VSC en configuración back to back respecto de la generación de armónicos y la versatilidad en el procesamiento de potencia. Se justifica la naturalidad de este tipo de conexión para el DCMC y se analizan las alternativas de control del conjunto. Se presenta un caso de estudio del accionamiento de un motor de inducción de media tensión con control directo de par adaptado a $M P C$. Finalmente en el capítulo 5 se realiza un análisis de un sistema $W E C S B 2 B$ en el camino de potencia con un generador de imanes permanentes y se elabora una estrategia para el control del generador y la interfaz a red teniendo en cuenta las restricciones impuestas por los códigos de operación de las redes.

En el capítulo 6 se elaboran algunas conclusiones y se presenta la perspectiva de trabajos futuros. 



\section{Capítulo 2}

\section{$\underline{\text { Convertidores Multinivel }}$}

\subsection{Introducción}

La capacidad operativa de un convertidor de potencia depende básicamente de su topología circuital y de los dispositivos semiconductores que lo componen. Los convertidores multinivel son estructuras topológicas que permiten aumentar los niveles de tensión de operación por encima de la tensión máxima admisible de los dispositivos de conmutación. Cada topología multinivel posee características propias que dependen de la estructura de interconexión de los dispositivos. Estas características se relacionan con restricciones en los estados de conmutación y las transiciones entre estados de conmutación, con las pérdidas y con el control de variables internas del mismo, generalmente las tensiones en los capacitores del bus de continua. Por otro lado, los esquemas de control de las variables externas (corriente, potencia, etc.) deben explotar las bondades de las topologías en términos de la minimización de la distorsión armónica de la tensión o de la corriente de salida. Existe una variedad de métodos de control de los convertidores multinivel que se adecuan en mayor o menor medida a estos requerimientos dependiendo de la topología considerada.

En este capítulo se realiza una revisión descriptiva de los dispositivos de conmutación y los convertidores electrónicos $D C / A C$. Se introduce la problemática de escalabilidad de los dispositivos por simple conexión en paralelo o en serie. Se presentan las tres topologías básicas multinivel y los principios que permiten ampliar sus límites operativos de tensión con respecto a los de las llaves de potencia individuales. Se presenta el convertidor multinivel con enclavamiento por diodos y se analizan sus 
características de funcionamiento, limitaciones y requerimientos de control particulares. Se mencionan los métodos de modulación existentes para este tipo de convertidores y se desarrolla un método de modulación vectorial para convertidores multinivel. También se propone un algoritmo generalizado para el control de balance de las tensiones del bus de continua, aplicable a convertidores con un número arbitrario de niveles. El esquema de control desarrollado se caracteriza en términos de sus límites de estabilidad y se muestra su desempeño en un convertidor de 5 niveles funcionando como compensador estático de potencia reactiva $[60][61][62][63]$.

\subsection{Clasificación de convertidores de potencia DC/AC}

Los inversores de potencia indirectos pueden clasificarse según el nivel de controlabilidad del estado de conducción de los dispositivos como convertidor de conmutación forzada (FCC: forced commutated converter) o convertidor conmutado por carga (LCC: load commutated converter). También, existen dos clases según el elemento almacenador de energía en el bus de continua, a saber: convertidor alimentado con tensión (VSC: voltage source converter) y convertidor alimentado con corriente (CSC: current source converter). En la Fig. 2.1(a) y (b) se muestran ambos tipos de convertidores trifásicos $F C C$ de dos niveles con almacenamiento mediante capacitor e inductor, respectivamente.

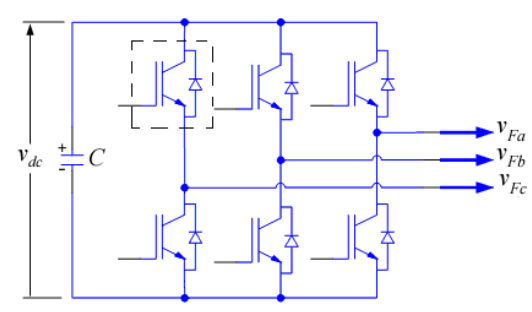

(a)

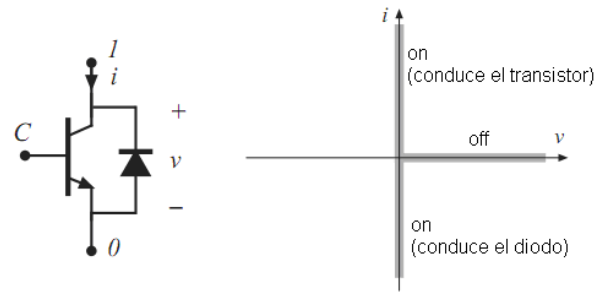

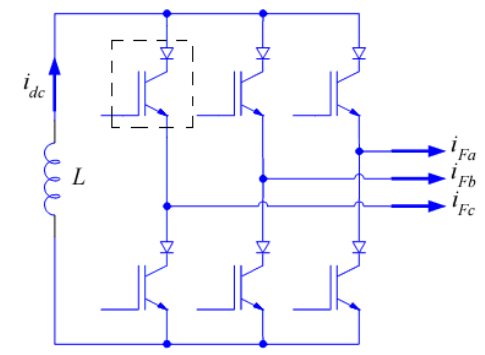

(b)

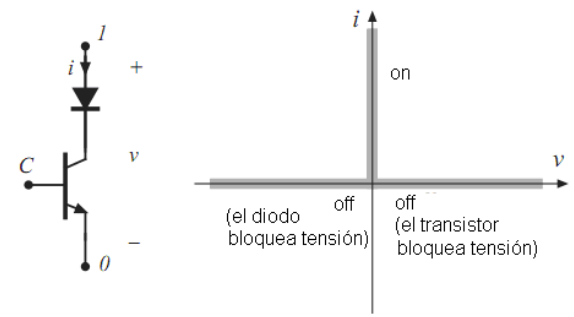

Figura 2.1. Inversores FCC según su elemento de almacenamiento. (a) VSC, (b) CSC. 
Con respecto a las ventajas del CSC puede mencionarse una mayor robustez debido principalmente a la forma constructiva y principio físico de funcionamiento del elemento almacenador [74][75] y su inmunidad ante cortocircuitos en los bornes de salida debido a que la corriente a través de las llaves se limita al valor de diseño de la del bus de continua. Como contrapartida, presenta altas pérdidas en el inductor de almacenamiento y sobre los dispositivos de conmutación, ya que la corriente circula por dos dispositivos (transistor y diodo). También, la implementación de las llaves con bloqueo bidireccional de tensión son inconvenientes pues no se fabrican integradas y por tanto es necesario realizar el montaje discreto de ambos dispositivos. En este sentido, si bien existe gran motivación para el desarrollo de dispositivos $I G B T$ con capacidad de bloqueo bidireccional (RB-IGBT) para aplicación en convertidores matriciales, los resultados aún son incipientes [76][77][78].

Inversamente, los VSC poseen menores pérdidas en el elemento de almacenamiento del bus de continua como en sus dispositivos de conmutación, en tanto que los dispositivos con conducción bidireccional de corriente son ampliamente aceptados y poseen gran popularidad en el mercado. Esto hace de estos convertidores la alternativa más utilizada en la mayor parte de las aplicaciones.

\subsubsection{Dispositivos de conmutación}

Desde la aparición del tiristor, la tecnología de dispositivos de conmutación ha logrado un empuje constante hacia mayores niveles de tensión y corriente, y mayor controlabilidad y facilidad de cambio de su estado de conducción. A diferencia del tiristor el cual posee sólo capacidad de control de puesta en conducción, los dispositivos a los que se hará mención son de conmutación forzada, esto es, su estado de conmutación no depende de las variables del circuito de salida y puede cambiarse su estado en forma arbitraria. Básicamente, existen dos tecnologías de conmutación forzada basadas en sus dispositivos de origen: el tiristor y el transistor bipolar. Las variantes de la tecnología del tiristor comienzan con el GTO hasta el IGCT para altas potencias y frecuencias de conmutación moderadas y las del transistor bipolar comienza con el desarrollo del transistor bipolar de potencia $(B P T)$ y la combinación de éste con el transistor de efecto de campo de compuerta aislada (MOSFET) culmina con el IGBT, más apropiado en el rango de potencias moderadas y altas frecuencias de conmutación. 
Los dispositivos de la familia del tiristor requieren circuitería adicional para el corte del dispositivo, tratándose entonces de dispositivos híbridos. En cambio, los dispositivos de la familia del transistor bipolar poseen una construcción integrada. En la Fig. 2.2 se simboliza la evolución cronológica de ambas familias de dispositivos.

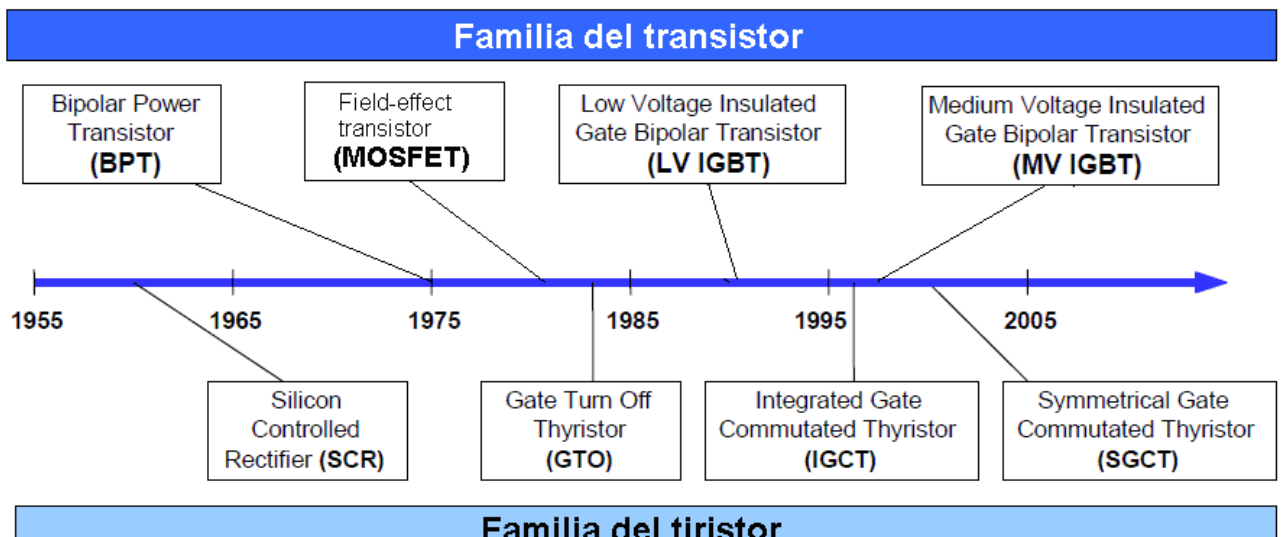

Figura 2.2. Evolución de los dispositivos de potencia

Otros dispositivos que no han logrado una penetración significativa en el mercado, pero que merecen mención son:

1) ETO (emmiter turn-off thyristor)

2) MCT (MOS commutated thyristor)

3) IEGT (injection-enhanced gate transistor)

Con referencia a nuevos materiales semiconductores se encuentran en estudio algunas alternativas al silicio. Materiales con gap de energía superiores como el diamante, nitruro de galio $(\mathrm{GaN})$ y carburo de silicio $(\mathrm{SiC})$ están siendo intensamente investigados [4][79]. Sin embargo algunas de estas tecnologías son muy incipientes o bien sus procesos de fabricación no han adquirido madurez suficiente como para competir comercialmente con sus pares de silicio.

Para evaluar la utilización de un dispositivo dado, resulta necesario tener en cuenta los parámetros principales que intervienen en un inversor de potencia: tensión inversa máxima, corriente máxima y pérdidas por conducción y conmutación. La Fig. 2.3(a) muestra la evolución de características de los dispositivos mencionados y su ubicación 
en función de los niveles de corriente, tensión y potencia. Por otra parte, en la Fig. 2.3(b) se ubican en función de la corriente, tensión y frecuencia de trabajo.

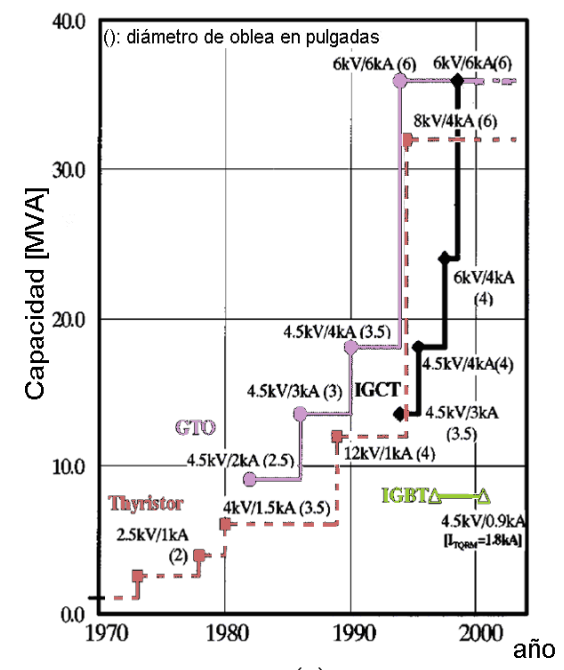

(a)

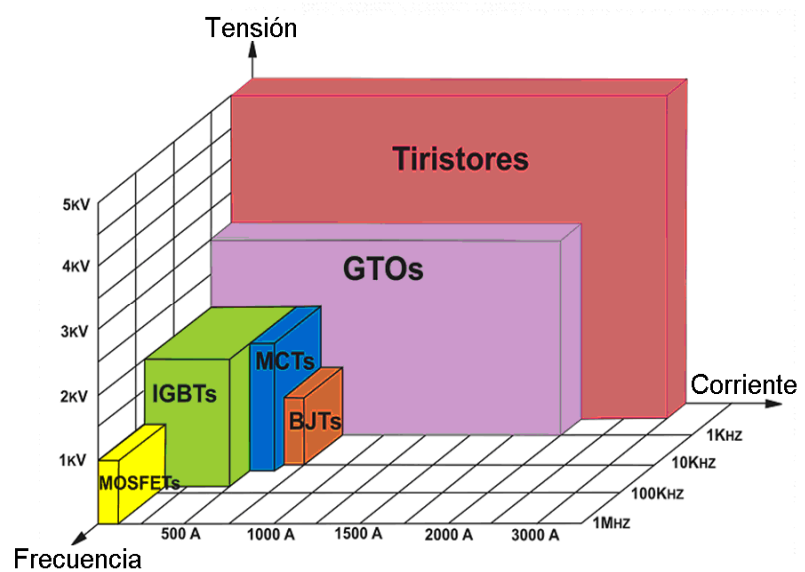

(b)

Figura 2.3. (a) Evolución cronológica de la capacidad operativa de los dispositivos, (b) Ubicación de las tecnologías respecto de la capacidad operativa y frecuencia de conmutación.

Para una dada potencia, tensión de trabajo y frecuencia de conmutación del convertidor es posible dimensionar los dispositivos según su ubicación en los gráficos anteriores teniendo en cuenta márgenes de seguridad apropiados [80].

En virtud de lo anterior y con referencia a las topologías de la Fig. 2.1 se desprende que las llaves del VSC deben ser capaces de bloquear la tensión del bus de continua, en tanto que las del CSC deben soportar la corriente del inductor de almacenamiento. Para extender dichos límites de corriente o tensión puede acudirse a estrategias de conexión de dispositivos en paralelo o en serie, respectivamente. Sin embargo, esta práctica presenta dificultades dependiendo del tipo de dispositivo y la variable que se desea extender. La afinidad de un dispositivo dado para aplicar esta técnica depende de su estructura y parámetros propios y se caracteriza a través de su "escalabilidad". Particularmente con respecto a la conexión en serie de dispositivos (de interés para la operación de los $V S C$ ), debe mencionarse que debido a disparidad en los procesos de fabricación, la conexión en serie provoca desbalance de las tensiones de bloqueo. El desbalance estático se debe a las diferencias entre las corrientes de fuga de los dispositivos, la cual a su vez depende de la temperatura y de la tensión de trabajo, mientras que el desbalance dinámico se origina en: (a) diferencias en el comportamiento en conmutación de los dispositivos y (b) diferencias en las señales de disparo de los 
dispositivos por parte del controlador. La Tabla 2.1 muestra los efectos y sus causas alrededor de esta problemática [8].

Tabla 2.1. Efectos y causas de desbalance de tensión en dispositivos conectados en serie

\begin{tabular}{|l|l|l|}
\hline \multicolumn{1}{|c|}{ Tipo } & \multicolumn{2}{|c|}{ Causas de desbalance de tensión } \\
\hline $\begin{array}{l}\text { Distribución } \\
\text { estática de } \\
\text { tensión }\end{array}$ & \multicolumn{1}{|c|}{$\begin{array}{l}\text { Corriente de pérdidas estática } \\
\text { Temperatura de la juntura }\end{array}$} \\
\hline \multirow{2}{*}{$\begin{array}{l}\text { Distribución } \\
\text { dinámica de } \\
\text { tensión }\end{array}$} & Disp. & $\begin{array}{l}\text { - Retardo a la activación } \\
\text { - Retardo al corte } \\
\text { - Recuperación de la carga del } \\
\text { diodo en antiparalelo } \\
\text { - Temperatura de la juntura }\end{array}$ \\
\cline { 2 - 3 } & Driver & $\begin{array}{l}\text { - Retardo del driver a la activación } \\
\text { - Retardo del driver a la desactivación } \\
\text { drivery el pin de control del dispositivo }\end{array}$ \\
\cline { 2 - 3 } & \multicolumn{2}{|c|}{$\begin{array}{l}\text { Desapareamiento entre dispositivos conectados } \\
\text { en serie }\end{array}$} \\
\hline
\end{tabular}

Frecuentemente, estos inconvenientes obligan a utilizar circuitos auxiliares de ecualización y/o controles activos de conmutación de las llaves (AOC: active overvoltage clamping). Sin embargo, estas acciones complican el montaje, aumentan las pérdidas y tienden a disminuir la confiabilidad del sistema.

Las topologías multinivel constituyen una alternativa a la conexión en serie de los dispositivos para aumentar la tensión de operación de los convertidores. Esto es posible, gracias al efecto de enclavamiento de las tensiones de bloqueo de los dispositivos de conmutación a las tensiones de nivel. De esta manera es posible prescindir del uso de redes de ecualización y de circuitos $A O C$. Como ventaja adicional, estos convertidores son capaces de sintetizar tensiones de salida escalonadas reduciendo así el contenido armónico de las formas de onda de salida.

\subsubsection{Convertidores multinivel}

Si bien la topología de puente $H$ en conexión serie fue introducida previamente como método de síntesis de una forma de tensión escalonada en una aplicación en baja potencia [81], el inicio formal de los convertidores de potencia multinivel comienza con 
el trabajo de Nabae et al. en 1981 [82] donde se presenta el convertidor con conexión al punto neutro del bus de continua (NPC: neutral point clamp). Esta topología permite duplicar la tensión de trabajo del convertidor con respecto a la tensión de bloqueo máxima de los dispositivos de conmutación evitando el uso de redes de ecualización y/o sistemas activos de control de conmutación de llaves en serie. Además sintetiza una forma de onda de tensión escalonada mejorando la distorsión armónica y el $d V / d t$ de la tensión de salida. La intensa investigación de dicha topología ha hecho del NPC una topología ampliamente difundida por varios fabricantes, y a la vez aceptada en el ambiente industrial. La generalización de dicho convertidor, el convertidor multinivel con enclavamiento por diodos (DCMC: diode clamped multilevel converter) junto con el de capacitores flotantes (FCMC: flying capacitor multilevel converter) y el de celdas en cascada ( $C H B$ : cascaded H-bridge) constituyen las tres topologías generalizadas de los convertidores multinivel. La Fig. 2.4 muestra una columna de cada una de dichas topologías para 5 niveles. Paralelamente se han desarrollado topologías alternativas híbridas con cantidad reducida de componentes que mejoran el factor de utilización de las llaves y otros aspectos de importancia para la implementación y el desarrollo comercial. Cabe destacar que todas las topologías multinivel son capaces de sintetizar tensiones con múltiples niveles sin dispositivos conectados en serie y sin transformadores. Por otro lado, la síntesis de la tensión de salida mediante múltiples niveles y la implementación de estrategias de modulación permiten reducir la distorsión armónica como así también las reactancias de filtrado a la salida.

La topología de capacitores flotantes (Fig. 2.4(a)) fue introducida por Meynard y Foch en 1992 [83][84]. Ésta hace uso de los capacitores internos de cada columna para enclavar las tensiones de todas las llaves de conmutación y a la vez generar los distintos niveles de tensión de salida. El bus de continua común a todas las columnas posiciona a esta topología para la conexión back to back, de gran interés en aplicaciones de acondicionamiento de potencia y en accionamiento de máquinas. Por otra parte, la gran cantidad de capacitores origina redundancia de las tensiones de columna (una tensión de columna puede ser sintetizada por más de una combinación de conmutación), ofreciendo gran flexibilidad para el manejo del balance de las tensiones en los capacitores. Sin embargo, la excesiva cantidad de estados posibles dificulta la selección de las secuencias de conmutación para esta finalidad. Otra desventaja que presenta el 
convertidor $F C M C$ es que requiere una estrategia especial para la precarga de los capacitores de enclavamiento en el arranque.

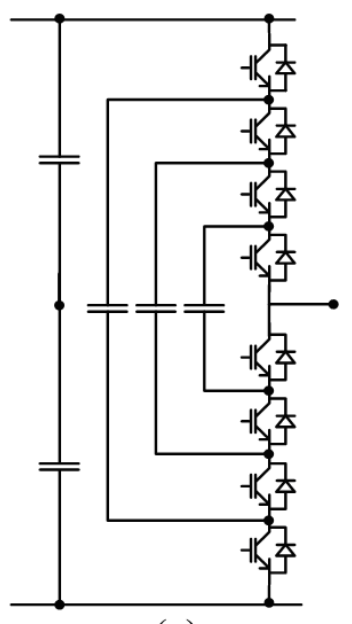

(a)

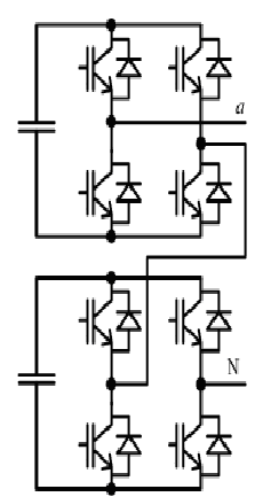

(b)

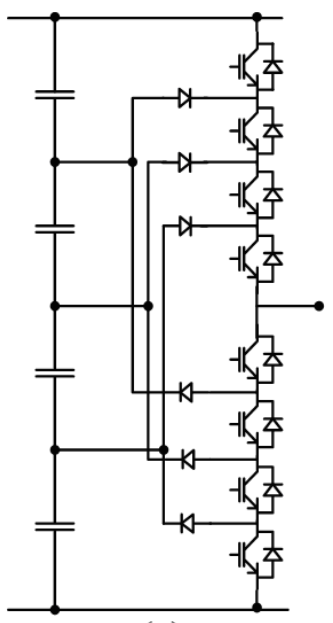

(c)

Figura 2.4. Topologías generalizadas de convertidores multinivel (5 niveles), (a) FCMC, (b) CHB, (c) DCMC.

La Fig. 2.4(b) muestra una columna del inversor de celdas en cascada, la cual consiste en la conexión en serie de puentes monofásicos [85]. Según se observa, cada celda puede forzar en su salida tres tensiones diferentes. Para un convertidor con $N$ celdas idénticas se logran $(2 N+1)$ niveles de tensión de columna. La gran ventaja de esta topología es la modularidad tanto en las celdas de potencia como en el control de las mismas ya que no existe interacción entre celdas ni entre columnas, promoviendo una implementación sencilla e independiente del control de cada una de ellas. Si bien a priori este convertidor no posee mejor relación entre el número de dispositivos y niveles sintetizados que sus competidores, es posible aumentar dicha relación definiendo valores distintos de tensión de bus para cada celda ( $C H B$ híbrido). La gran desventaja de esta topología consiste en la necesidad de disponer de buses de continua aislados, lo cual obliga a utilizar transformadores y rectificadores con varios secundarios, $\mathrm{o}$ convertidores $D C / D C$ aislados. Esta característica también impide su conexión en configuración back to back. Por ello, dicha topología es adecuada principalmente en aplicaciones donde no existe transferencia de potencia activa, como compensadores estáticos de reactivo y de armónicas [86]. 


\subsection{Convertidor multinivel con enclavamiento por diodos}

El convertidor multinivel enclavamiento por diodos es la topología generalizada del convertidor $N P C$. La Fig. 2.5 muestra una columna del DCMC de 5 niveles. El mismo está constituido por las llaves activas con sus diodos de rueda libre, los diodos de enclavamiento y el bus $D C$ con sus tomas intermedias.

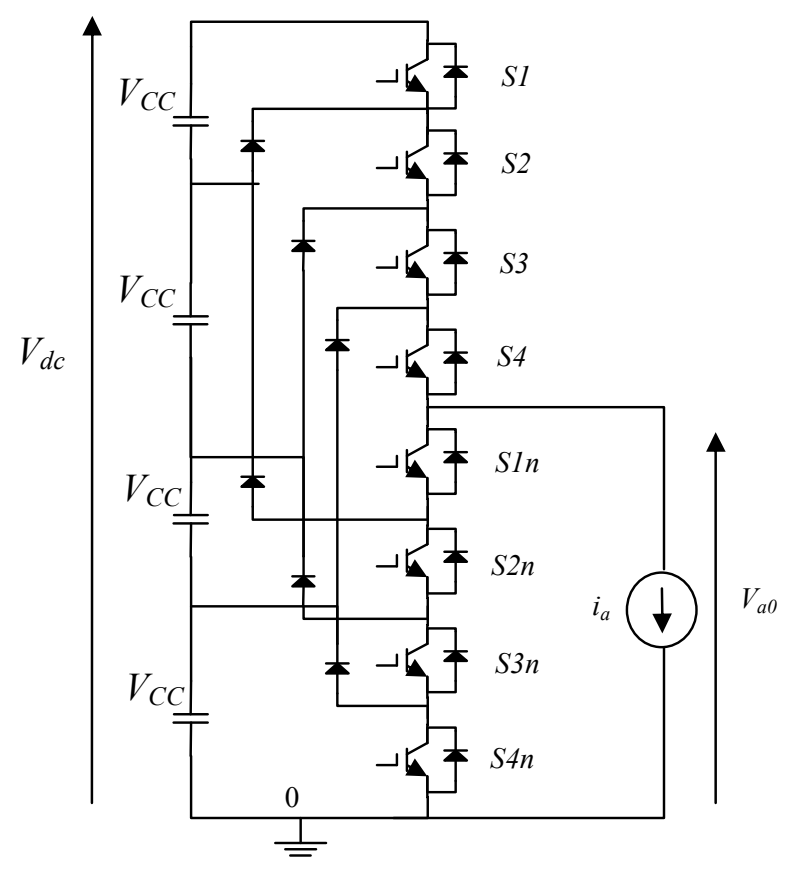

Figura 2.5. Convertidor DCMC de 5 niveles y lógica de comando de las llaves.

A los efectos de que cada columna sea capaz de forzar la tensión en su salida, los dispositivos deben comandarse de a pares complementarios. Esto significa que existirán $(N-1)$ señales de comando para cada columna, $(S 1, S 2, S 3 \ldots S(N-1))$ y sus complementos $(S 1 n, S 2 n, S 3 n$ y $S(N-1) n)$ distribuidas según se indica en la Fig. 2.5. La Tabla 2.2 expresa la tensión de salida de la columna en función de las señales de comando de las llaves, mientras que las Figs. 2.6(a-j) muestran todas las combinaciones de conmutación enumeradas en la Tabla 2.2 para ambos sentidos de circulación de corriente. El estado de conducción de los dispositivos se denota mediante un "1" y un "0" en el gate de cada uno de ellos indicando que el dispositivo se encuentra encendido y apagado, respectivamente. Para cada combinación de conmutación, la activación de las llaves activas actúa sobre la polarización de los diodos de enclavamiento y de rueda libre definiendo un único camino de circulación para la corriente de carga, así como un 
único valor de tensión $V_{a 0}$. Los dispositivos a través de los cuales circula la corriente de carga se indican en color verde claro. Aquellas combinaciones que no aparecen en la tabla ni en la Fig. 2.6 no son válidas pues dan lugar a tensiones $V_{a 0}$ que dependen del sentido de circulación de la corriente de salida $i_{s}$, generan cortocircuitos en los capacitores del bus o bien producen tensiones de bloqueo excesivas sobre los dispositivos de conmutación [87].

Tabla 2.2. Señales de comando de las llaves y tensiones de columna

\begin{tabular}{|c|c|c|c|c|c|c|c|c|}
\hline \multirow{2}{*}{$\begin{array}{c}\text { tensión de } \\
\text { columna }\end{array}$} & \multicolumn{9}{|c|}{ Estado de las laves } \\
\cline { 2 - 9 } & $\mathrm{S} 1$ & $\mathrm{~S} 2$ & $\mathrm{~S} 3$ & $\mathrm{~S} 4$ & $\mathrm{~S} 1 \mathrm{n}$ & $\mathrm{S} 2 \mathrm{n}$ & $\mathrm{S} 3 \mathrm{n}$ & $\mathrm{S} 4 \mathrm{n}$ \\
\hline 4Vcc & 1 & 1 & 1 & 1 & 0 & 0 & 0 & 0 \\
\hline 3Vcc & 0 & 1 & 1 & 1 & 1 & 0 & 0 & 0 \\
\hline 2Vcc & 0 & 0 & 1 & 1 & 1 & 1 & 0 & 0 \\
\hline $\mathrm{Vcc}$ & 0 & 0 & 0 & 1 & 1 & 1 & 1 & 0 \\
\hline 0 & 0 & 0 & 0 & 0 & 1 & 1 & 1 & 1 \\
\hline
\end{tabular}

Como se puede apreciar de la Tabla 2.2 sólo existe una combinación de conmutación de columna para la síntesis de cada valor de tensión en su salida, es decir que la topología no posee redundancia en las tensiones de columna.

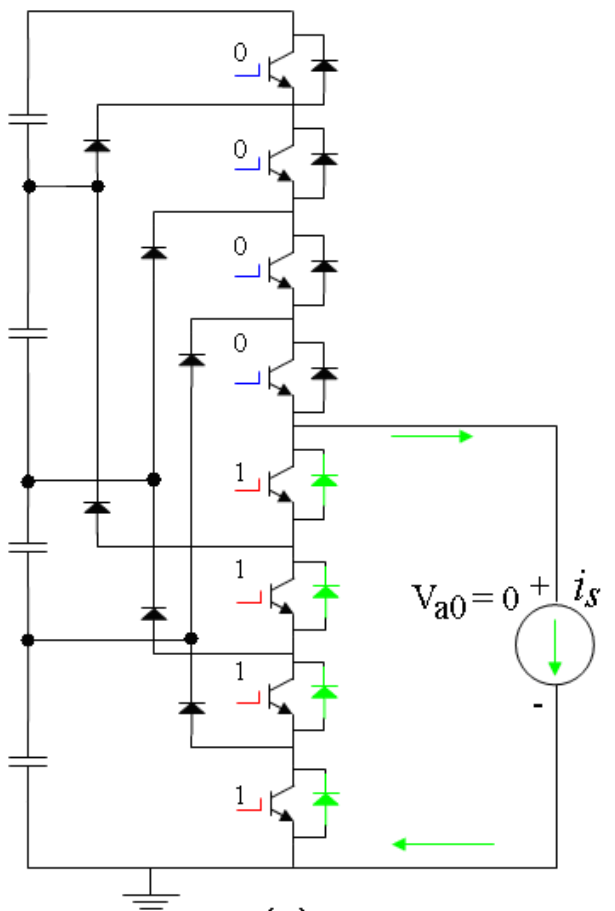

(a)

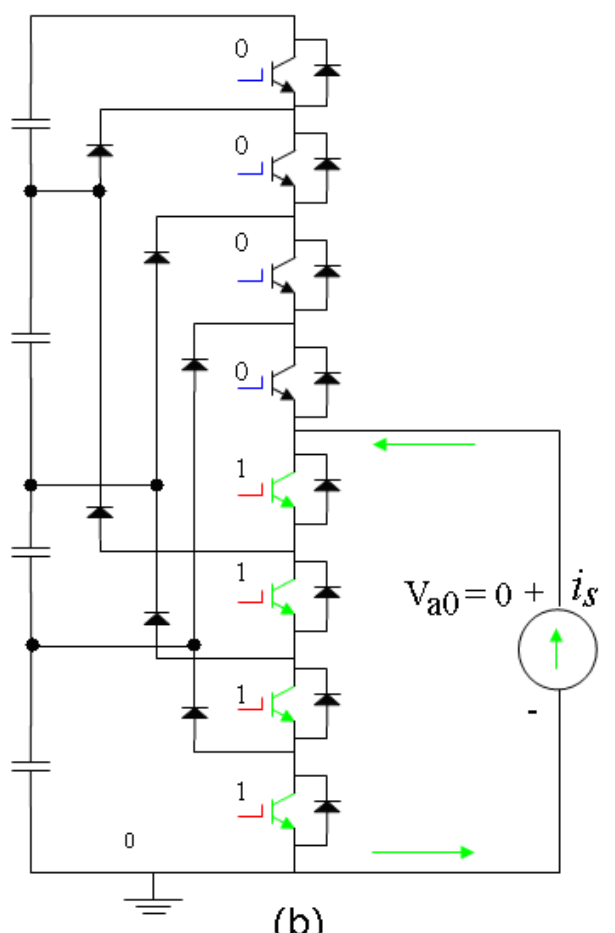

(b) 

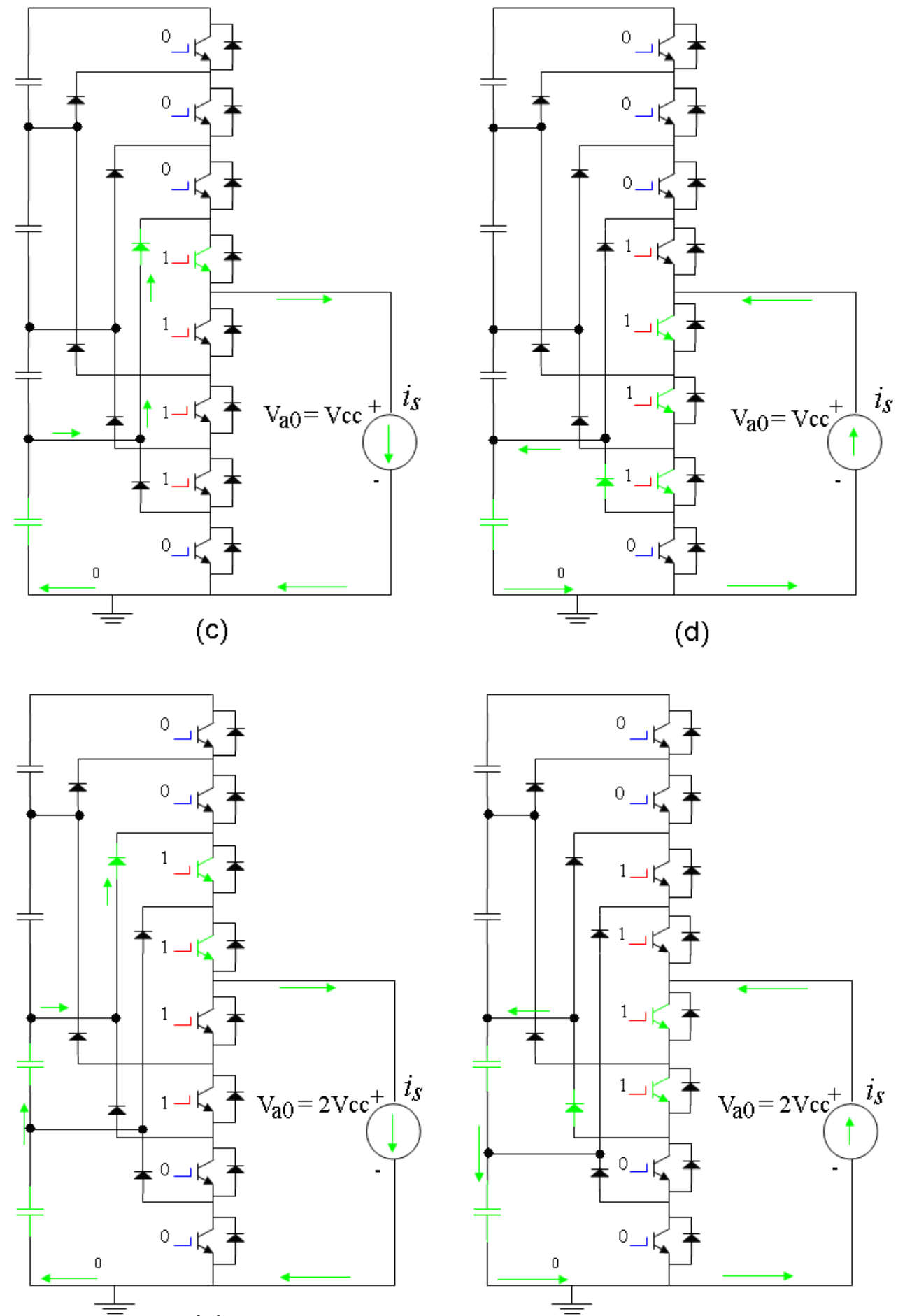

(e)

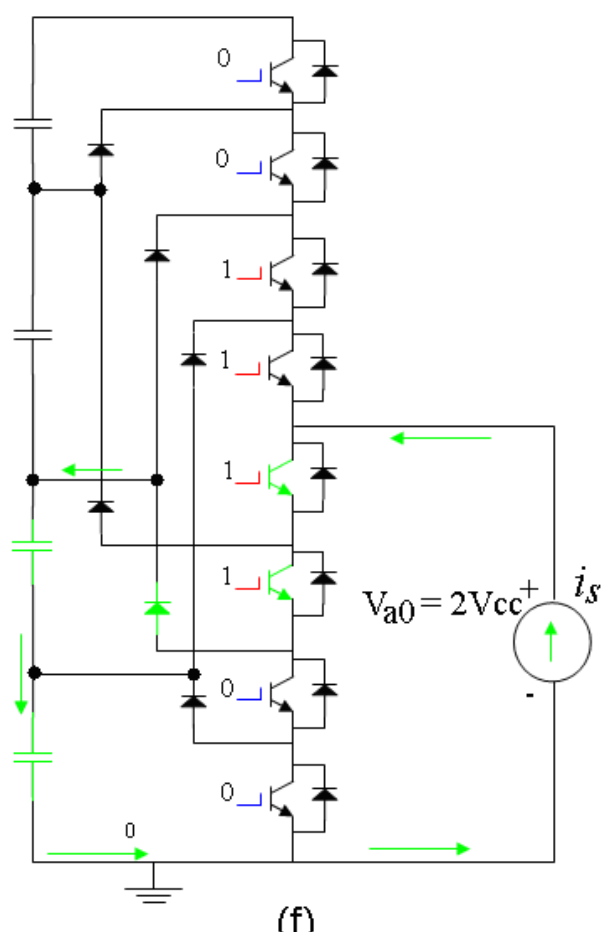




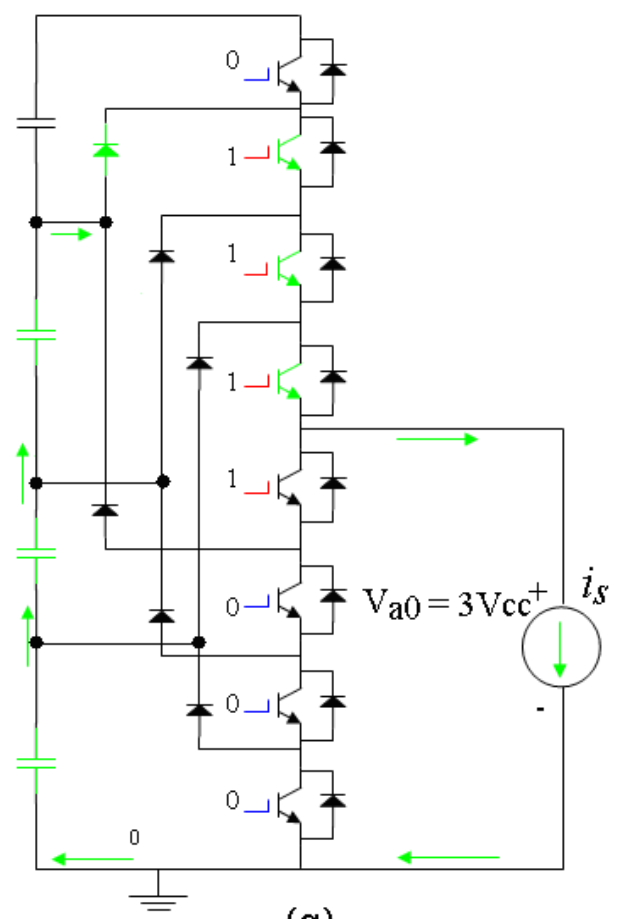

(g)

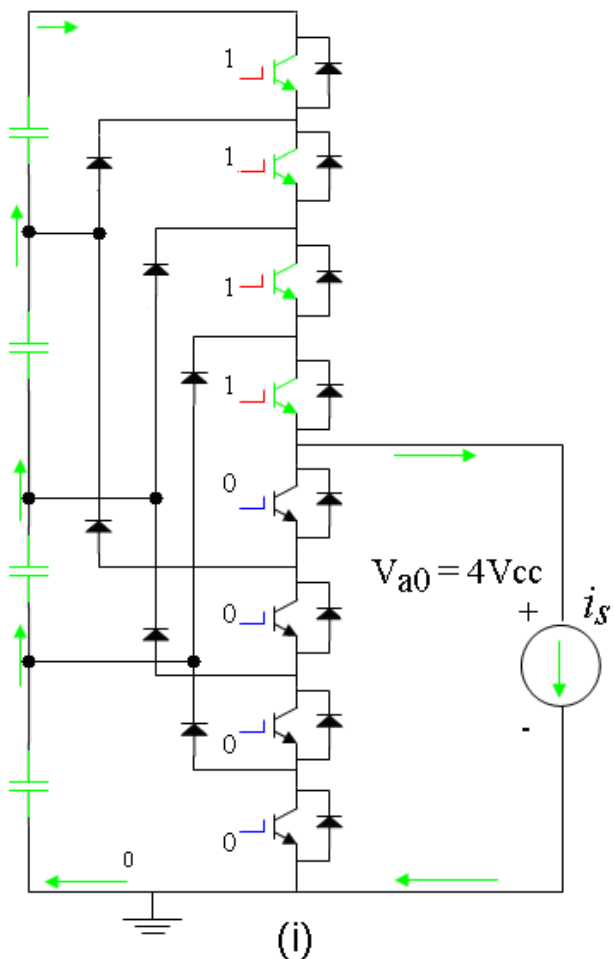

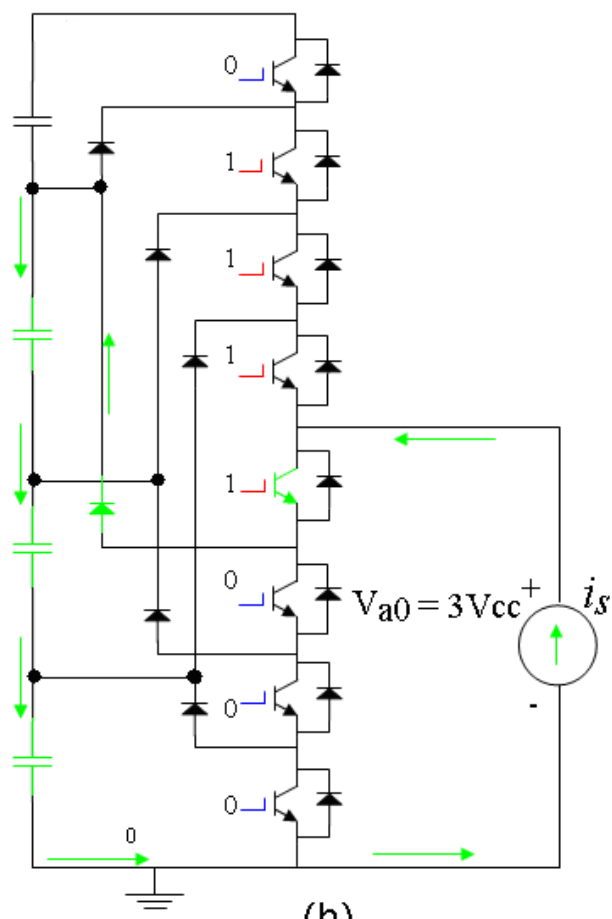

(h)

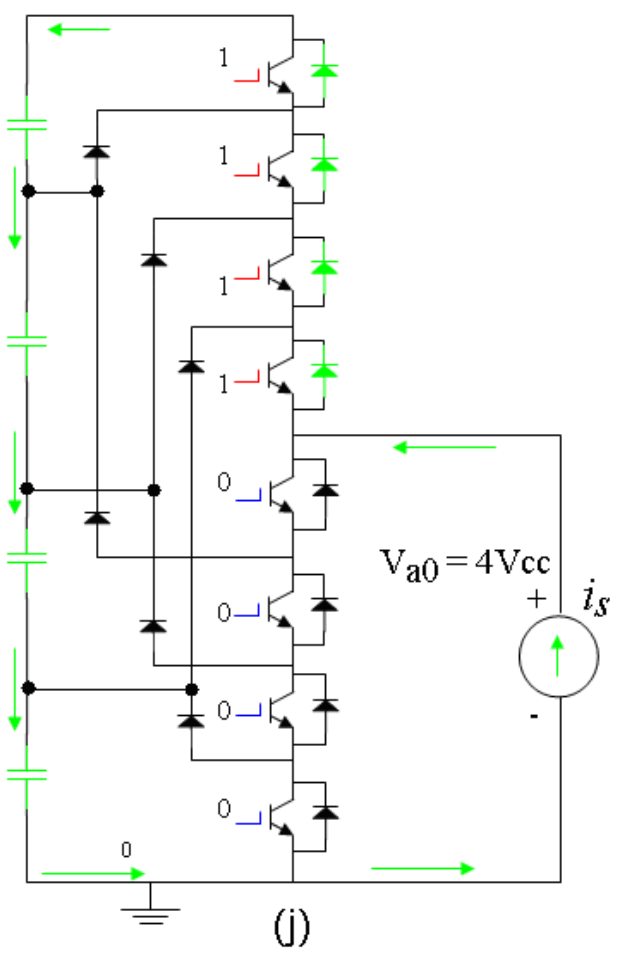

Figura 2.6. Combinaciones de conmutación posibles para la columna del convertidor DCMC de 5 niveles y circulación de la corriente en ambos sentidos.

El esquema de conmutación mencionado permite sintetizar un modelo funcional de las columnas del convertidor mediante el uso de llaves con múltiples entradas y una salida. 
La equivalencia entre dichas llaves y el comando de los dispositivos de conmutación se muestra en la Fig. 2.7(a), para un convertidor de 5 niveles y la tensión de salida (Fig. 2.7(b)) de una columna respecto del negativo del bus $D C$ y considerando constantes e idénticas las tensiones sobre los capacitores.

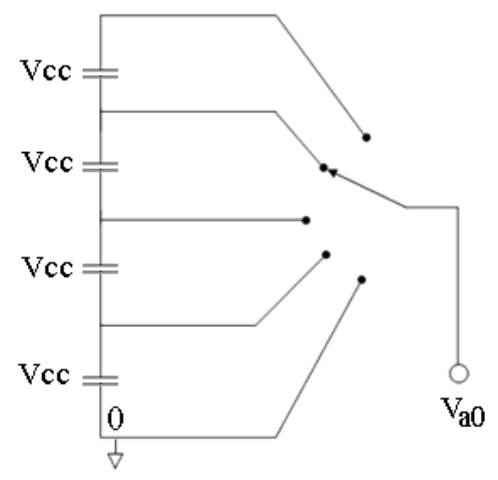

(a)

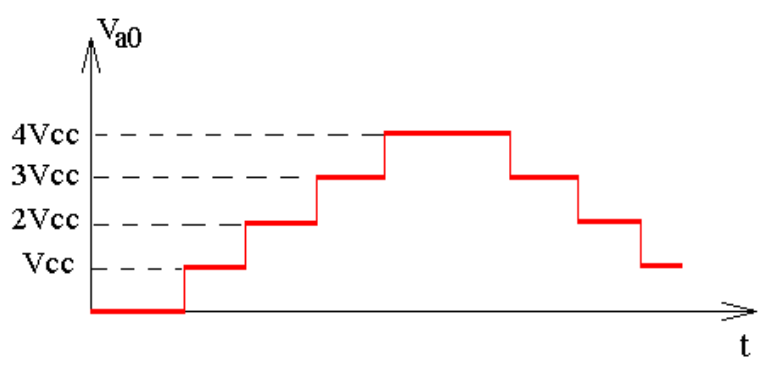

(b)

Figura 2.7. (a) Equivalente funcional de una columna del convertidor (b) Tensión de columna.

Con respecto a las transiciones entre un estado de conmutación y el siguiente puede decirse que el enclavamiento de los transistores internos es unilateral, lo cual requiere un cuidado especial en las transiciones entre estados de conmutación sucesivos. Esta restricción estriba en el efecto de limitación que ejercen los diodos de enclavamiento a las tensiones de los nodos intermedios del bus de continua. Sin embargo, dada la topología de la columna, sólo los dispositivos que se encuentran en los extremos superior e inferior se enclavan en forma directa, mientras que los dispositivos internos sufren enclavamiento indirecto [83][88]. Esto determina que, ante variaciones de las tensiones de columna en más de un nivel (salto múltiple), las tensiones de bloqueo de las llaves puedan exceder el valor permitido durante el transitorio de conmutación. La Fig. 2.8 ejemplifica la transición múltiple de una columna para el convertidor con diodos de enclavamiento de 3 niveles (el razonamiento es extensible a mayor número de niveles). Se observa que el diodo de enclavamiento $\mathrm{D}_{2}$ exige que $V_{2} \leq V_{C C}$. Esto implica que $V_{l} \geq V_{C C}$. Estas relaciones indican que la topología no asegura el enclavamiento de todos los dispositivos a la tensión de nivel $V_{C C}$, sino que sólo aseguran una cota máxima para los dispositivos de los extremos $\mathrm{S} 1$ y $\mathrm{SN}-1 \mathrm{n}$ (Fig. 2.5), en este caso $Q_{1}$ y $Q_{4}$. 


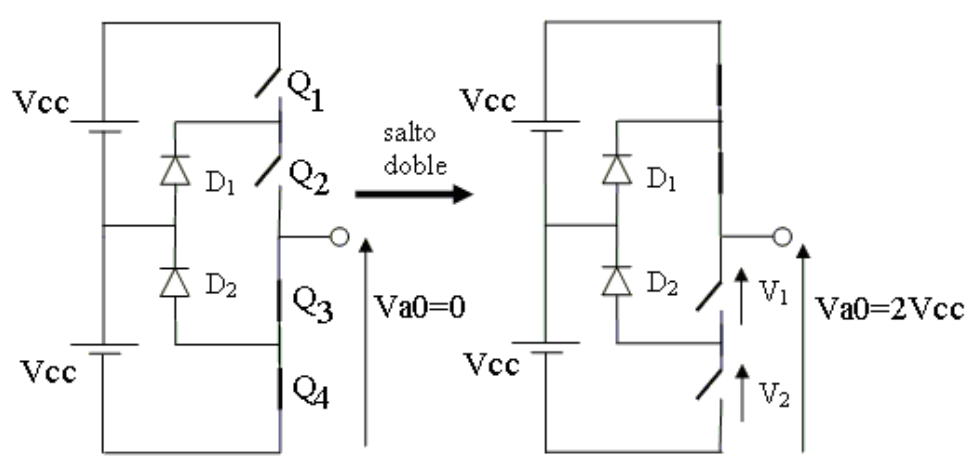

Figura 2.8. Transición múltiple de columna del convertidor con diodos de enclavamiento (3 niveles)

En [89][90][91][92] se analiza el funcionamiento del convertidor DCMC durante la conmutación. Se advierte que para asegurar el enclavamiento correcto de las tensiones de bloqueo sobre las llaves es preciso realizar la conmutación en forma secuencial entre niveles sucesivos. Esto significa que una columna del convertidor no puede desplazarse en más de un nivel del bus a la vez, constituyendo una regla restrictiva para la selección de las transiciones de estado de las llaves. Esta restricción tiene carácter general para la topología con enclavamiento por diodos.

El convertidor $D C M C$ trifásico comprende tres columnas conectadas a un único bus de capacitores. En este caso las tres columnas del inversor aportan corriente a la totalidad de los capacitores del bus de continua. Esto implica que las variaciones de tensión resultantes en cada capacitor depende de las tres corrientes de línea, de los nodos del bus donde se inyectan y de la ventana de tiempo considerada. Como ya se ha mencionado, a diferencia de la topología con capacitores flotantes, el DCMC no dispone de redundancia en las tensiones de columna para atacar el problema del balance. Sin embargo, una alternativa explorada consiste en la utilización de la redundancia en las tensiones de línea [93]. Dicha redundancia consiste en que una dada terna de tensiones de línea puede sintetizarse, en general, por más de una terna de tensiones de columna.

La evaluación de la topología $D C M C$ respecto de sus pares multinivel en términos de la cantidad de componentes de almacenamiento de energía y su naturalidad topológica para la conexión $B 2 B$, resulta favorable para su aplicación dentro de las temáticas propuestas al principio de la tesis. 


\subsection{Modulación y control de convertidores multinivel}

En la literatura pueden encontrarse cuatro clases de moduladores de tensión para convertidores multinivel: control del vector espacial, modulación sub-armónica con portadoras desplazadas, modulación por programación de ángulos y modulación vectorial (Fig. 2.9). Todas ellas son adaptaciones de técnicas conocidas para inversores de dos niveles y poseen características particulares respecto a requerimientos de velocidad de conmutación de los dispositivos, flexibilidad para funcionamiento a frecuencia variable, complejidad de implementación.

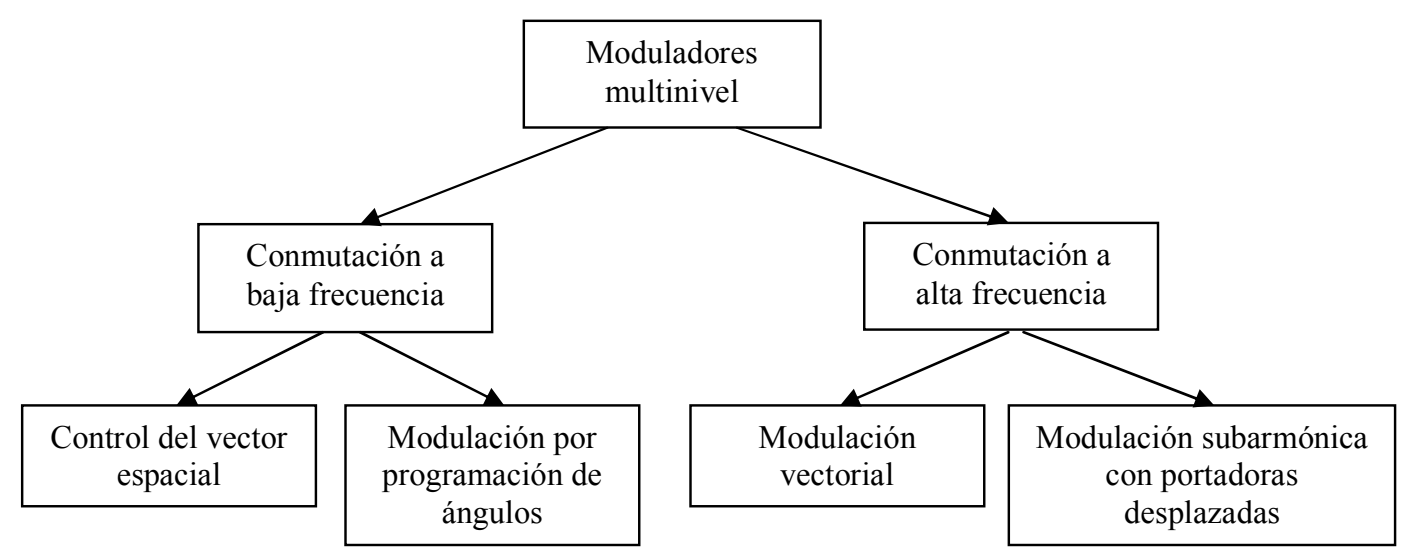

Figura 2.9. Métodos de modulación de inversores multinivel

La técnica de control del vector espacial introducida en [94] consiste en aplicar un dado vector durante un período de muestreo. Este algoritmo es extremadamente simple pues aplica durante un tiempo fijo el vector más cercano al vector de referencia. Esta selección se realiza a partir del vector de referencia y de un patrón de zonas definidas por hexágonos ubicados sobre el mapa de vectores sintetizables por el convertidor. El método obtiene buenos resultados de distorsión de la forma de onda de tensión para convertidores con un número de niveles elevado.

La técnica de modulación por programación de ángulos es similar a la aplicada para convertidores de dos niveles, pero considerando el conjunto de valores discretos de amplitud de las tensiones de columna para la eliminación u optimización de los armónicos. Como en el caso de dos niveles, los ángulos se calculan off-line y se programan las tablas de búsqueda en la memoria de lectura del sistema. Este método 
encuentra aplicación en sistemas que operan con frecuencia y amplitud constantes como sistemas de alimentación ininterrumpida, pero son inadecuados para aplicaciones que requieren frecuencia variable y gran ancho de banda [23].

La técnica de modulación con portadoras desplazadas fue la primera en extenderse desde los convertidores de dos niveles a los multinivel. Esto se debe a la simplicidad conceptual y también a su facilidad de implementación. Consiste en la comparación de la señal de referencia (modulante) con portadoras asociadas a los niveles de tensión de salida. Dentro de este esquema están las variantes de portadoras desplazadas en fase y en nivel y cada una posee rendimientos diferentes dependiendo de la topología de convertidor sobre la que se aplica. La desventaja de este método consiste en que resulta difícil introducir modificaciones para atacar problemas adicionales propios de los convertidores multinivel, como la reducción de la frecuencia de conmutación, reducción de las tensiones de modo común y el balance de tensión de los capacitores. Como consecuencia, los grados de libertad que inherentemente ofrecen estas topologías no pueden ser explotados de forma conveniente.

\subsubsection{Modulación vectorial en convertidores multinivel}

El método de modulación vectorial presenta versatilidad cuando se requiere rápida respuesta dinámica y/o operación a frecuencia variable, tales como accionamientos y acondicionamiento de potencia. Desde el punto de vista de la implementación, la técnica de modulación vectorial presenta tres aspectos interesantes [95]:

- Posibilidad de optimización de la cantidad de conmutaciones.

- Mayor aprovechamiento del bus de continua.

- Implementación natural en sistemas digitales.

Asimismo, ofrece flexibilidad para el ataque de problemáticas propias del convertidor y de optimización de variables adicionales. Sin embargo, la extensión del método en convertidores multinivel requiere administrar convenientemente todos los estados de conmutación disponibles, $\mathrm{y}$, si bien la selección de los mismos no resulta sencilla dado el gran número de combinaciones, especialmente cuando $N$ aumenta, se ha demostrado en [96] que puede evitarse gran parte de esta carga definiendo el estado del convertidor 
mediante un vector representado sobre un sistema de coordenadas hexagonales. El algoritmo comprende la ejecución de dos etapas:

1) Síntesis de las tensiones de línea deseadas en los bornes de salida del convertidor.

2) Optimización de parámetros adicionales mediante la selección de las tensiones de columna.

Para entender esto, en la Fig. 2.10 se introducen los conceptos de vector de tensiones de línea y sus correspondientes realizaciones en tensiones de columna para el caso de un convertidor $D C M C$ de 5 niveles.

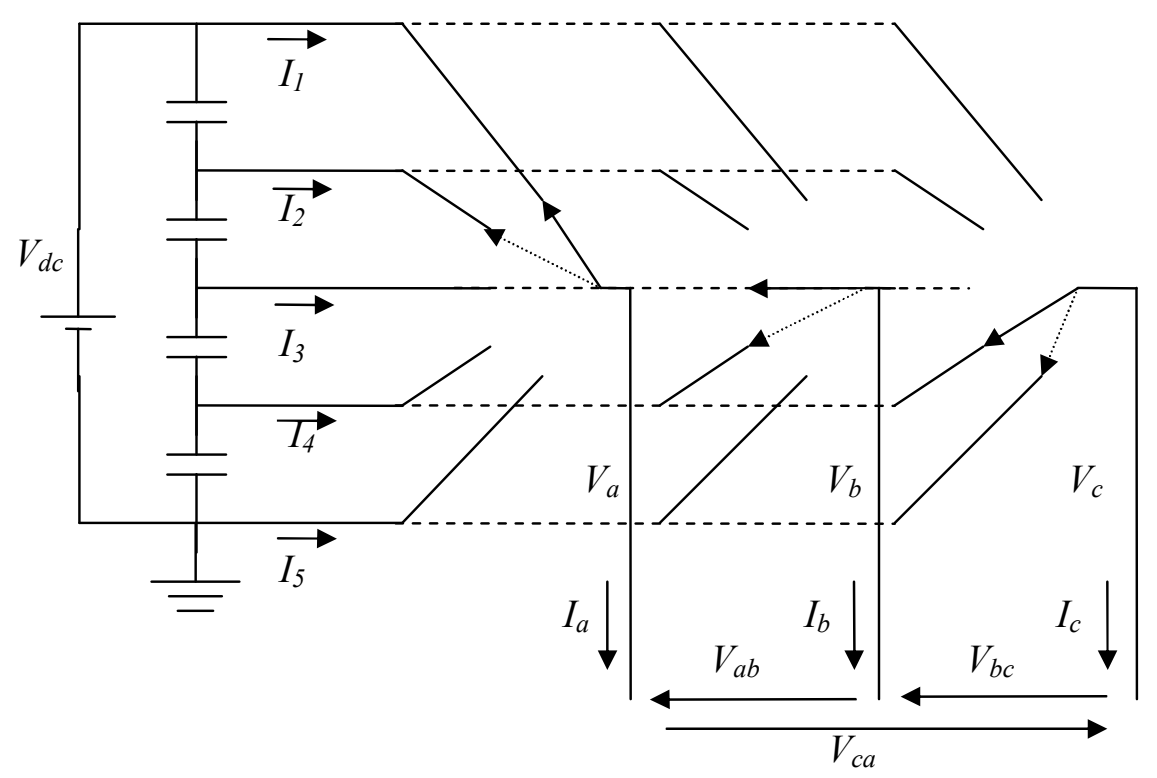

Figura 2.10. Redundancia de tensiones de línea y flujo de corrientes sobre los capacitores del bus DC.

Se puede apreciar que los dos estados de conmutación especificados en la Fig. 2.10 (sólido y punteado) sintetizan una misma terna de tensiones de línea. Puede concluirse entonces que una terna de tensiones de línea posee una o más realizaciones de tensiones de columna. Es decir que, desde el punto de vista de una carga trifásica trifilar, tales realizaciones son equivalentes. El número de realizaciones de un vector de línea depende del número de ternas redundantes y éste, a su vez, de la mayor tensión de línea de la terna referida a la tensión total del bus (índice de modulación). En particular, si quisiéramos sintetizar una terna $\left(V_{a b}, V_{b c}, V_{c a}\right)$ tal que alguna de las tensiones fuera $V_{d c}$ 
(tensión máxima), sólo existiría una combinación de conmutación posible, o lo que es lo mismo, no existiría redundancia en dicha terna. Por otra parte, si bien distintas realizaciones son equivalentes desde el punto de vista de una carga trifilar, no lo son desde el punto de vista del flujo de corriente en los nodos intermedios del divisor capacitivo. Esto indica que las realizaciones de un dado vector repercuten de forma diferente en la tensión de cada capacitor del divisor del bus, sugiriendo que dicha redundancia constituye una acción de control posible para atacar el problema del desbalance de tensión en los capacitores.

\subsubsection{Desarrollo del método}

La representación más difundida del vector espacial considera las tensiones de fase de la carga $V_{a n}, V_{b n} y V_{c n}$, las cuales, mediante una transformación de coordenadas $T_{\alpha \beta 0}$, se transforman al sistema coordenado $\alpha-\beta-0$ (2.1). Las componentes en dicho sistema, $V_{\alpha}$, $V_{\beta}$ y $V_{0}$ se calculan a partir de dicha transformación, según:

$$
\left[\begin{array}{l}
V_{\alpha} \\
V_{\beta} \\
V_{0}
\end{array}\right]=T_{\alpha \beta 0}\left[\begin{array}{l}
V_{a n} \\
V_{b n} \\
V_{c n}
\end{array}\right] \text { donde }: T_{\alpha \beta 0}=\frac{2}{3}\left[\begin{array}{ccc}
1 & -\frac{1}{2} & -\frac{1}{2} \\
0 & \frac{\sqrt{3}}{2} & -\frac{\sqrt{3}}{2} \\
\frac{1}{\sqrt{2}} & \frac{1}{\sqrt{2}} & \frac{1}{\sqrt{2}}
\end{array}\right]
$$

Esta representación permite caracterizar los estados de los interruptores de un convertidor mediante los vectores que resultan de dichas combinaciones. En la Fig. 2.12(a) se presenta el esquema de un inversor de dos niveles alimentado con fuente de tensión. En este caso cada conmutador posee sólo dos posiciones posibles: $m=1 \mathrm{y}$ $m=0$. De este modo el convertidor define seis vectores activos sobre el plano $\alpha-\beta$, como se muestra en la Fig. 2.11(b). 


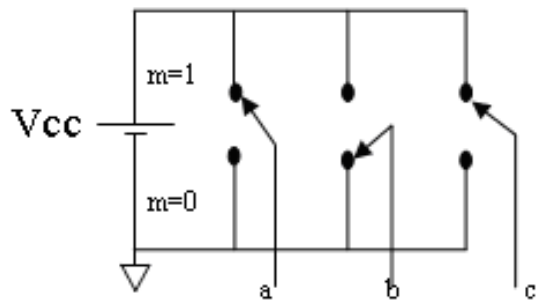

(a)

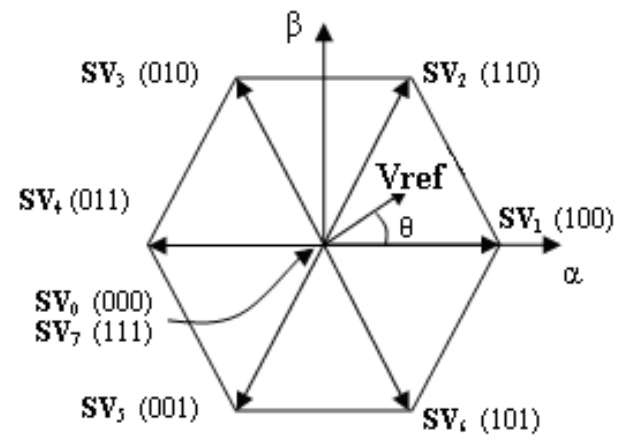

(b)

Figura 2.11. (a) Convertidor de 2 niveles, (b) Mapa de vectores sintetizables.

Cada vector está identificado en la Fig. 2.11(b) según el valor de $m$ para cada fase $\left(m_{a} m_{b} m_{c}\right)$. También se observa que hay dos combinaciones $\left(\begin{array}{l}\mathbf{S V}_{0} \\ \text { y }\end{array} \mathbf{S V}_{7}\right)$ que representan al vector nulo. Una combinación consiste en las tres columnas conectadas a $0 \mathrm{~V}(m=0)$ mientras que la otra, se obtiene con las tres conectadas a $V_{C C}(m=1)$. Esto constituye un grado de libertad que se utiliza habitualmente para minimizar la cantidad de conmutaciones sobre los dispositivos. En síntesis, un convertidor de dos niveles ofrece $2^{3}=8$ combinaciones posibles de los dispositivos de conmutación, mientras que el número de vectores sintetizables es igual a 7 .

El proceso de modulación vectorial consiste en aproximar el vector de referencia $\mathbf{V}_{\text {ref }}$ mediante la promediación de los tres vectores más cercanos a $\mathbf{V}_{\text {ref }}$ dentro del periodo de promediación $T_{S}$

$$
\mathbf{V}_{\text {ref }}=d_{1} \mathbf{V}_{1}+d_{2} \mathbf{V}_{2}+d_{3} \mathbf{V}_{3}
$$

donde $d_{1}, d_{2}$ y $d_{3}$ representan los ciclos de trabajo de los vectores $\mathbf{V}_{1}, \mathbf{V}_{2}$ y $\mathbf{V}_{3}$, los cuales se determinan en forma directa a partir del ángulo $\theta$.

En la Fig. 2.12 se representa un periodo de promediación. Este se divide en los tres tiempos de activación correspondientes a cada uno de los vectores empleados. $\mathbf{V}_{1}, \mathrm{y} \mathbf{V}_{2}$ son los vectores activos adyacentes a $\mathbf{V}_{\text {ref, }}$, mientras que $\mathbf{V}_{3}$ corresponde a un vector nulo. 


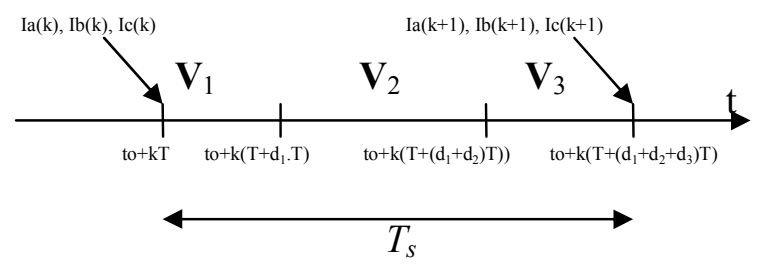

Figura 2.12. Periodo de promediación.

Considerando el convertidor de tres niveles mostrado en la Fig. 2.13, se observa que cada conmutador posee tres posiciones posibles. El mapa de vectores sintetizables se amplía significativamente, como se muestra en la Fig. 2.14. En el extremo de cada vector están indicadas las posiciones de los conmutadores de columna que los sintetizan. Se observa en la misma figura, que algunos vectores se obtienen con más de una combinación de estados de los interruptores. Precisamente todos los vectores que conforman el hexágono interno poseen dos combinaciones posibles, mientras que el vector nulo posee tres. Esta característica de redundancia puede resumirse en 19 vectores diferentes con $3^{3}=27$ combinaciones posibles.

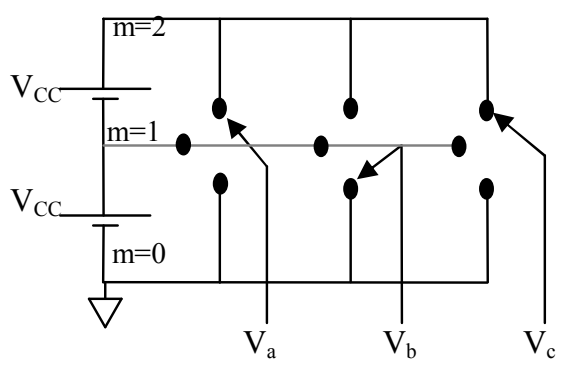

Figura 2.13. Convertidor de 3 niveles NPC.

En general, para un convertidor de $N$ niveles, existen $N^{3}$ maneras diferentes de combinar las tensiones de columna, mientras que el número de vectores sintetizables está dado por la siguiente expresión [95]:

$$
L=1+6 \sum_{i=1}^{N-1} i
$$




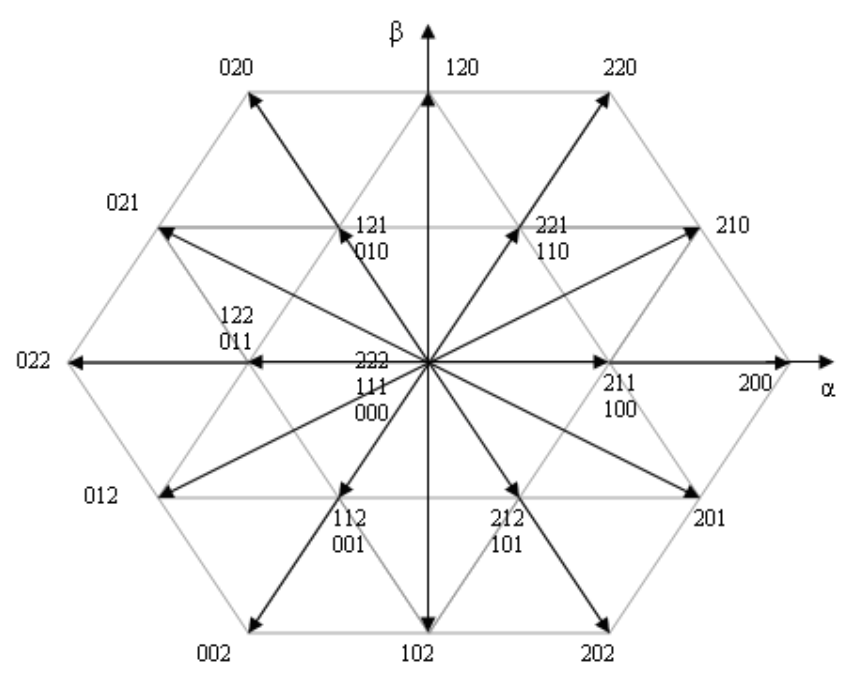

Figura 2.14. Mapa de vectores para un DCMC de tres niveles.

Se desprende que a medida que $N$ aumenta el número de anillos hexagonales y la cantidad de estados redundantes también aumenta. A diferencia del convertidor de dos niveles, los tres vectores a promediar no pueden determinarse sólo a partir de $\theta$, sino que también interviene el módulo del vector de referencia. Esto contribuye a que la selección de los vectores a promediar no sea una tarea sencilla. En tal sentido puede observarse en la Fig. 2.14 que la representación del vector a sintetizar mediante componentes en cuadratura $\alpha$ y $\beta$ no permite una identificación inmediata de los vectores más cercanos. En [97] se propone un método de descomposición de los hexágonos internos y se aplica a un convertidor NPC. Si bien la propuesta es general para un número arbitrario de niveles, la cantidad de cálculos necesarios aumenta dramáticamente a medida que $N$ crece. Sin embargo, se ha demostrado en [98] para 3 niveles y en [96] para $N$ niveles, que es posible aliviar la carga computacional utilizando un sistema de referencia hexagonal, el cual se ajusta a la distribución geométrica de los vectores sintetizables por el convertidor. Dicho marco de referencia presenta ventajas ya que la representación de los mismos resulta en componentes enteras.

\subsubsection{Coordenadas hexagonales}

En un convertidor multinivel, la identificación de los estados de conmutación para la síntesis de un vector de referencia dado requiere analizar en forma conjunta el módulo y el ángulo de dicho vector con relación a los vectores sintetizables por el convertidor. La 
multiplicidad de estados en estas topologías hace que esto no sea una tarea sencilla. Incluso, la transición entre estados consecutivos impone un condicionamiento adicional relacionado con las conmutaciones entre nodos adyacentes del bus de continua. La representación de los estados del convertidor en un sistema de ejes coordenados hexagonales (a diferencia del sistema coordenado $\alpha-\beta-0$ ) ofrece una representación más coherente de los estados del convertidor y permite identificar en forma inmediata los tres vectores más cercanos $(N T V)$. En primer lugar, se propone la representación del vector espacial mediante tensiones de línea en lugar de tensiones de fase. Esta elección permite identificar directamente las tensiones a la salida del convertidor alcanzando una representación independiente del tipo de carga y su conexión. Podemos representar un vector $\mathbf{V}$ en el espacio $(\mathbf{X}, \mathbf{Y}, \mathbf{Z})$ cuyas componentes sean las tensiones de línea de un sistema trifásico. Dicho vector puede expresarse,

$$
\mathbf{V}=V_{a b} \mathbf{X}+V_{b c} \mathbf{Y}+V_{c a} \mathbf{Z} \quad \text { o de otra forma: } \mathbf{V}=\left[\begin{array}{lll}
V_{a b} & V_{b c} V_{c a}
\end{array}\right]^{T}
$$

Dado que las tensiones consideradas $\left(V_{a b}, V_{b c}, V_{c a}\right)$ se cierran sobre una malla, su suma resulta nula. Luego, el vector definido en (2.3) está contenido en el plano $X+Y+Z=0$. En particular, una terna trifásica de tensiones sinusoidales y balanceadas puede representarse mediante un vector de referencia:

$$
\mathbf{V}_{\text {ref }}=V_{\text {llpico }}\left[\begin{array}{lll}
\cos (\omega t) & \cos (\omega t-2 \pi / 3) & \cos (\omega t+2 \pi / 3)
\end{array}\right]^{T}
$$

donde $V_{\text {llpico }}$ es la tensión de línea pico y $\omega$ la pulsación del sistema. Puede demostrarse que este vector describe una trayectoria circular con velocidad angular $\omega$ sobre el plano $X+Y+Z=0$, como se muestra en la Fig. 2.15.

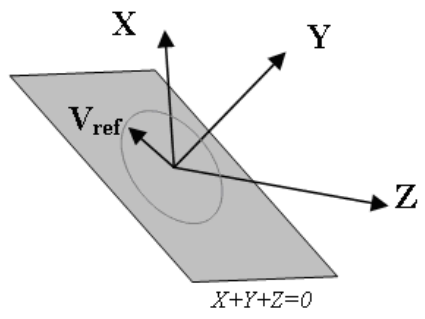

(a)

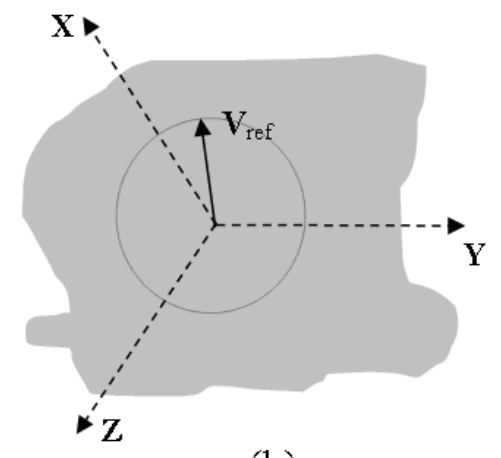

(b)

Figura 2.15. (a) Sistema ortogonal de tensiones, (b) Proyección de los ejes coordenados y del vector de referencia sobre el plano $X+Y+Z=0$ 
Para todos los valores de $\omega$ el vector definido en (2.4) está contenido en un plano y por lo tanto, su representación puede efectuarse con dos componentes en lugar de tres.

Para obtener una representación en coordenadas hexagonales, se propone una terna de vectores $\mathbf{u}, \mathbf{v}$ y $\mathbf{z}$ cuyas componentes se definen como:

$$
[\mathbf{u}, \mathbf{v}, \mathbf{z}]=\left[\begin{array}{ccc}
V_{C C} & 0 & V_{C C} \\
0 & V_{C C} & V_{C C} \\
-V_{C C} & -V_{C C} & V_{C C}
\end{array}\right]
$$

Luego se define una transformación de sistema de coordenadas que permita pasar del sistema $(\mathbf{X}, \mathbf{Y}, \mathbf{Z})$ a otro denominado $(\mathbf{g}, \mathbf{h}, \mathbf{y})$, en donde $\mathbf{u}$ se transforme en el versor $\mathbf{g}=\left[\begin{array}{lll}1 & 0 & 0\end{array}\right]^{\mathrm{T}}, \mathbf{v}$ en el $\mathbf{h}=\left[\begin{array}{lll}0 & 1 & 0\end{array}\right]^{\mathrm{T}}$ y $\mathbf{z}$ en el $\mathbf{y}=\left[\begin{array}{lll}0 & 0 & 1\end{array}\right]^{\mathrm{T}}$. Esta transformación posee la propiedad de que los vectores sintetizables por un convertidor multinivel con tensiones de nivel $V_{C C}$ poseen componentes enteras sobre los ejes $g$ y $h$, y nulos sobre el eje $y$. La matriz de transformación de coordenadas $T$ se obtiene resolviendo la igualdad siguiente:

$[\mathbf{g}, \mathbf{h}, \mathbf{y}]=\left[\begin{array}{lll}1 & 0 & 0 \\ 0 & 1 & 0 \\ 0 & 0 & 1\end{array}\right]=T[\mathbf{u}, \mathbf{v}, \mathbf{z}]=T\left[\begin{array}{ccc}V_{C C} & 0 & V_{C C} \\ 0 & V_{C C} & V_{C C} \\ -V_{C C} & -V_{C C} & V_{C C}\end{array}\right]$ con: $\quad T=\left[\begin{array}{ccc}V_{C C} & 0 & V_{C C} \\ 0 & V_{C C} & V_{C C} \\ -V_{C C} & -V_{C C} & V_{C C}\end{array}\right]^{-1}=\frac{1}{3 V_{C C}}\left[\begin{array}{ccc}2 & -1 & -1 \\ -1 & 2 & -1 \\ 1 & 1 & 1\end{array}\right]$

Como ejemplo se considera un convertidor de cuatro niveles en el que los conmutadores se ubican según se indica en la Fig. 2.16. La representación del vector en el espacio $(\mathbf{g}, \mathbf{h}, \mathbf{y})$ se calcula aplicando la transformación $T$ :

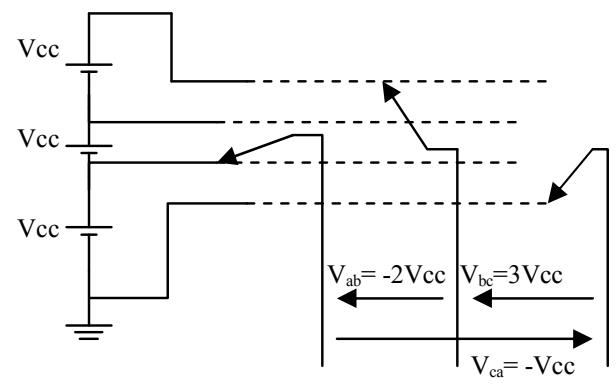

Figura 2.16. Convertidor de 4 niveles. 


$$
\left[\begin{array}{l}
V_{g} \\
V_{h} \\
V_{y}
\end{array}\right]=\frac{1}{3 V_{C C}}\left[\begin{array}{ccc}
2 & -1 & -1 \\
-1 & 2 & -1 \\
1 & 1 & 1
\end{array}\right]\left[\begin{array}{c}
-2 V_{C C} \\
3 V_{C C} \\
-V_{C C}
\end{array}\right]=\left[\begin{array}{c}
-2 \\
3 \\
0
\end{array}\right]
$$

Dado que la componente en la dirección $y$ es nula para cualquier terna de tensiones de línea, la matriz de transformación $T$ puede reducirse a:

$$
T^{\prime}=\frac{1}{3 V_{C C}}\left[\begin{array}{ccc}
2 & -1 & -1 \\
-1 & 2 & -1
\end{array}\right]
$$

La matriz de transformación $T$ normaliza las componentes del vector de referencia con respecto a la tensión de nivel $V_{C C}$. De este modo, los vectores sintetizables por el convertidor en el plano $g$ - $h$ conforman los vértices de los triángulos de la Fig. 2.17, para el convertidor de 4 niveles. El mapa de vectores se extiende naturalmente a convertidores de $N$ niveles agregando anillos hexagonales. Por otro lado, como consecuencia de la normalización impuesta por la matriz de transformación, los vértices de los triángulos poseen coordenadas enteras, como indican los pares coordenados $(g, h)$ al lado de cada vértice. Una terna trifásica de tensiones genera, en el plano $g$ - $h$, un vector de referencia que rota a una velocidad angular igual a la pulsación del sistema.

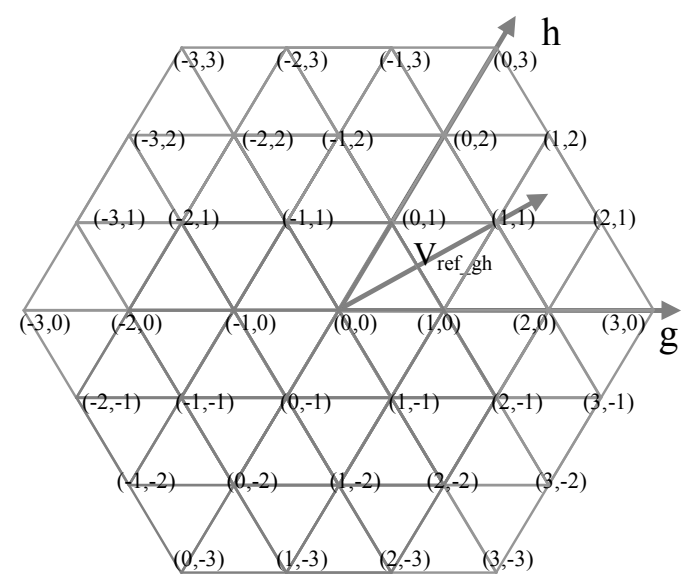

Figura 2.17. Mapa de vectores para cuatro niveles.

Aplicando la transformación de coordenadas $T^{\prime}$, obtenemos el vector de referencia $\mathbf{V}_{\text {ref } g h}$ en términos de sus componentes:

$$
\mathbf{V}_{\text {ref_gh }}=T^{\prime} \mathbf{V}_{\text {ref }}=\left[\begin{array}{ll}
V_{\text {ref_g }} & V_{\text {ref_h }}
\end{array}\right]^{T}
$$


Luego, el modulador debe determinar las posiciones adecuadas de los conmutadores para sintetizar el vector de referencia $\mathbf{V}_{\text {ref_gh }}$, mediante la expresión (2.2). El método comprende dos etapas:

1. Determinación de los tres vectores más cercanos al vector de referencia (NTV: Nearest Three Vectors) que pueden ser sintetizados por el convertidor.

2. Cálculo de los ciclos de trabajo de cada vector.

\subsubsection{Búsqueda de los NTV}

La Fig. 2.18 muestra una ampliación del primer sextante de la Fig. 2.17. Se observa el vector de referencia a sintetizar y sus componentes en el sistema coordenado $g$ - $h$. El extremo del vector de referencia se encuentra dentro del paralelogramo definido por los vértices $(2,2),(1,2),(1,1),(2,1)$.

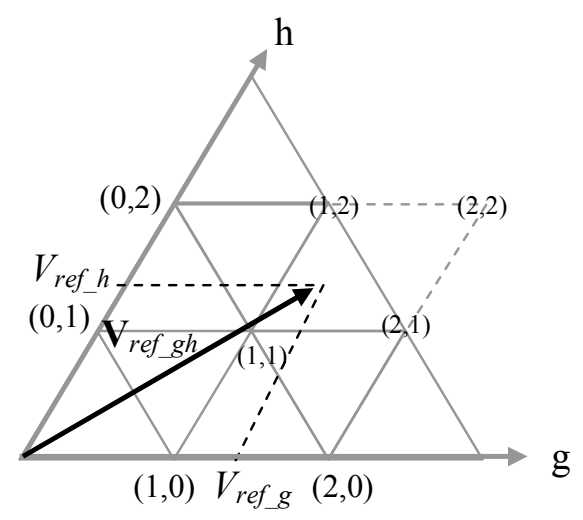

Figura 2.18. Vector de referencia y 4 vectores cercanos.

Es posible determinar los 4 vectores que definen el paralelogramo $(2,2),(1,2),(2,1)$, $(1,1)$ realizando el redondeo de las componentes del vector de referencia. Los vectores que definen dicho paralelogramo reciben sus nombres de acuerdo al tipo de redondeo de las componentes $g$ y $h$ según si el mismo es hacia arriba (upper) o hacia abajo (lower). Por ejemplo, el redondeo hacia arriba de la componente $V_{\text {ref } g}\left(V_{\text {ref } g} \uparrow\right)$ de la Fig. 2.18 es 2 , mientras que el redondeo hacia abajo $\left(V_{\text {ref } g} \downarrow\right)$ es 1 . Los vectores que definen dicho paralelogramo pueden expresarse en función de sus componentes: 


$$
\begin{array}{rll}
\mathbf{V}_{l u}=\left[\begin{array}{lll}
V_{\text {ref_g }} \downarrow & V_{\text {ref_h }} \uparrow
\end{array}\right]^{T} & \mathbf{V}_{u u}=\left[\begin{array}{lll}
V_{\text {ref_g }} \uparrow & V_{\text {ref_h }} \uparrow
\end{array}\right]^{T} \\
\mathbf{V}_{l l}=\left[\begin{array}{lll}
V_{\text {ref_g }} \downarrow & V_{\text {ref_h }} \downarrow
\end{array}\right]^{T} & \mathbf{V}_{u l}=\left[\begin{array}{lll}
V_{\text {ref_g }} \uparrow & V_{\text {ref_h }} \downarrow
\end{array}\right]^{T}
\end{array}
$$

En particular, para el vector de referencia mostrado en la Fig. 2.18:

$$
\mathbf{V}_{l u}=\left[\begin{array}{l}
1 \\
2
\end{array}\right] \quad \mathbf{V}_{u u}=\left[\begin{array}{l}
2 \\
2
\end{array}\right] \quad \mathbf{V}_{l l}=\left[\begin{array}{l}
1 \\
1
\end{array}\right] \quad \mathbf{V}_{u l}=\left[\begin{array}{l}
2 \\
1
\end{array}\right]
$$

Para conformar la terna de vectores más cercanos se eligen $\mathbf{V}_{l u} \mathrm{y} \mathbf{V}_{u l}$ en forma directa, mientras que la determinación del tercer vector precisa relacionar las componentes del vector de referencia con las de $\mathbf{V}_{u l}$ que se observan en la Fig. 2.19. Se define el número $S$ como:

$$
S=V_{r e f_{-} h}+V_{r e f_{-} g}-V_{u l_{-} g}-V_{u l_{-} h}
$$

donde $V_{u l \_g}, V_{u l \_} h, V_{\text {ref_g }}$ y $V_{\text {ref_h } h}$ son las componentes de los vectores $\mathbf{V}_{u l}$ y $\mathbf{V}_{\text {ref. }}$. Si $S<0$ significa que el vector de referencia se encuentra dentro del triángulo definido por los vértices $V_{l u}, V_{u l}$ y $V_{l l}$ y para $S>0$ el triángulo en cuestión es el definido por $V_{u l}, V_{l u} \mathrm{y}$ $V_{u u}$. Si $S=0$, el vector de referencia se encuentra sobre la recta que une los vértices $V_{l u} \mathrm{y}$ $V_{u l}$. En este caso los vectores promediados son $\mathbf{V}_{l u} \mathrm{y} \mathbf{V}_{u l}$. De esta manera, evaluando el signo de $S$ es posible hallar el tercer vector más cercano.

$$
\begin{aligned}
& \mathbf{V}_{1}=\mathbf{V}_{l u} \\
& \mathbf{V}_{2}=\mathbf{V}_{u l} \\
& \mathbf{V}_{3}=\left\{\begin{array}{lll}
\mathbf{V}_{l l} & \text { si } S<0 \\
\mathbf{V}_{u u} & \text { si } S>0 \\
0 & \text { si } S=0
\end{array}\right.
\end{aligned}
$$




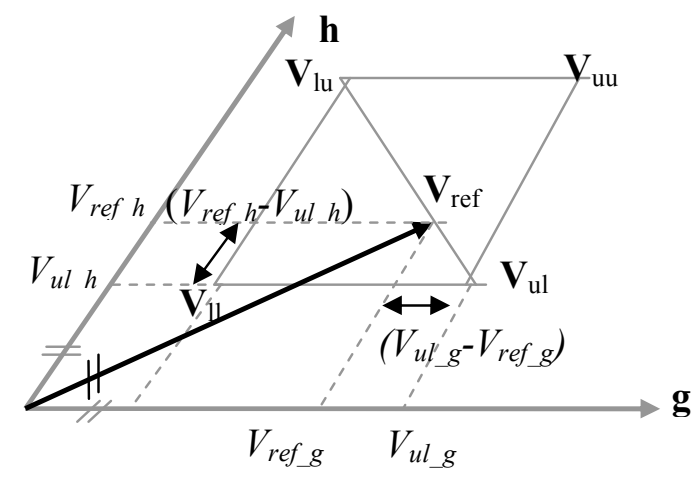

Figura 2.19. Determinación del tercer vector más cercano.

\subsubsection{Cálculo de los ciclos de trabajo}

El cálculo de los ciclos de trabajo se realiza utilizando la expresión (2.2) asignando a $\mathbf{V}_{1}, \mathbf{V}_{2}$ y $\mathbf{V}_{3}$ las coordenadas de $\mathbf{V}_{u l}, \mathbf{V}_{l u}$ y $\mathbf{V}_{3}$, determinadas mediante el mecanismo explicado en la sección anterior.

$$
\mathbf{V}_{r e f_{\text {_gh }}}=d_{u l} \mathbf{V}_{u l}+d_{l u} \mathbf{V}_{l u}+d_{3} \mathbf{V}_{3}
$$

donde $d_{u l}, d_{l u}$ y $d_{3}$ son los ciclos de trabajo de los vectores homónimos. De este modo, para el caso $\mathbf{V}_{3}=\mathbf{V}_{u u}$, expresando (2.6) en términos de las componentes de los vectores y teniendo en cuenta que $d_{3}=d_{u u}=1-d_{l u}-d_{u l}$ :

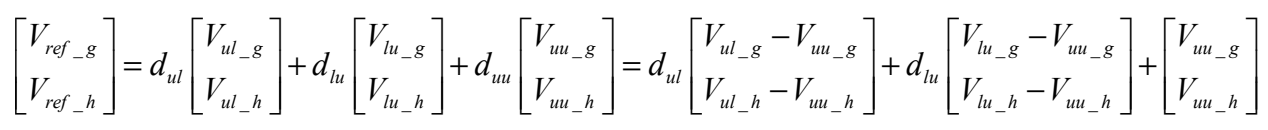

Además, como:

$V_{u l_{-} g}=V_{u u_{-} g}=\left[V_{r e f_{-} g}\right]+1, \quad V_{l u_{-} h}=V_{u u_{-} h}=\left[V_{r e f_{-} h}\right]+1, y \quad V_{u l_{-} h}-V_{l u_{-} h}=-1, V_{l u_{-} g}-V_{u u_{-} g}=-1$

donde [.] representa "parte entera", entonces resulta: 


$$
\left[\begin{array}{l}
V_{\text {ref_}} g \\
V_{\text {ref_h }}
\end{array}\right]=d_{u l}\left[\begin{array}{l}
0 \\
-1
\end{array}\right]+d_{l u}\left[\begin{array}{l}
-1 \\
0
\end{array}\right]+\left[\begin{array}{l}
{\left[V_{r^{2 e f} g}\right]+1} \\
{\left[V_{r^{e} f_{-} h}\right]+1}
\end{array}\right]
$$

de lo cual se desprende que:

$$
\begin{aligned}
& d_{u l}=1-\left(V_{r e f_{-} g}-\left[V_{r e f_{-} g}\right]\right) \\
& d_{l u}=1-\left(V_{r e f_{-} h}-\left[V_{r e f_{-} h}\right]\right) \\
& d_{3}=d_{u u}=\left(V_{r e f_{-} g}-\left[V_{r e f_{\_} g}\right]\right)+\left(V_{r_{e f} h}-\left[V_{r e f_{-} h}\right]\right)-1
\end{aligned}
$$

Para el caso $\mathbf{V}_{3}=\mathbf{V}_{l l}$ un razonamiento similar deriva en:

$$
\left[\begin{array}{l}
V_{\text {ref_g }} \\
V_{\text {ref_h }}
\end{array}\right]=d_{u l}\left[\begin{array}{l}
1 \\
0
\end{array}\right]+d_{l u}\left[\begin{array}{l}
0 \\
1
\end{array}\right]+\left[\begin{array}{c}
{\left[V_{\text {ref_g }}\right]} \\
{\left[V_{\text {ref_h }}\right]}
\end{array}\right]
$$

Y finalmente:

$$
\begin{aligned}
& d_{u l}=V_{r e f_{-} g}-\left[V_{r e f_{-} g}\right] \\
& d_{l u}=V_{r e f_{-} h}-\left[V_{r e f_{-} h}\right] \\
& d_{3}=d_{l l}=1-\left(V_{r e f_{-} g}-\left[V_{r e f_{-} g}\right]\right)-\left(V_{r e f_{-} h}-\left[V_{r e f_{-} h}\right]\right)
\end{aligned}
$$

Las ecuaciones (2.7) y (2.8) muestran la simplicidad de cálculo de los ciclos de trabajo: sólo es necesario separar la parte entera y la parte fraccionaria de las componentes del vector de referencia, tanto para determinar los vectores más cercanos como para calcular los tiempos de activación. En el cálculo de la modulación no son necesarias funciones trigonométricas ni tablas de búsqueda.

Utilizando la transformación inversa $T^{-1}$, es posible determinar las tensiones de línea a sintetizar en la salida del convertidor, para cada uno de los vectores $\mathbf{V}_{u l}, \mathbf{V}_{l u}$ y $\mathbf{V}_{3}$. Dado que cada terna de tensiones de línea puede lograrse, en general, con más de una terna de tensiones de columna, es necesario incorporar uno o más criterios adicionales para mapear las tensiones de línea a tensiones de columna. Esto permite adoptar un criterio que actúe sobre el balance del bus de continua, realizando un análisis de las variaciones de tensión sobre los capacitores en función de las corrientes de carga y de los estados de conmutación del convertidor. 


\subsection{Balance del bus de continua}

En la Fig. 2.20 puede observarse que la posición de los conmutadores determina la tensión de salida de cada columna con respecto al negativo del bus de continua.

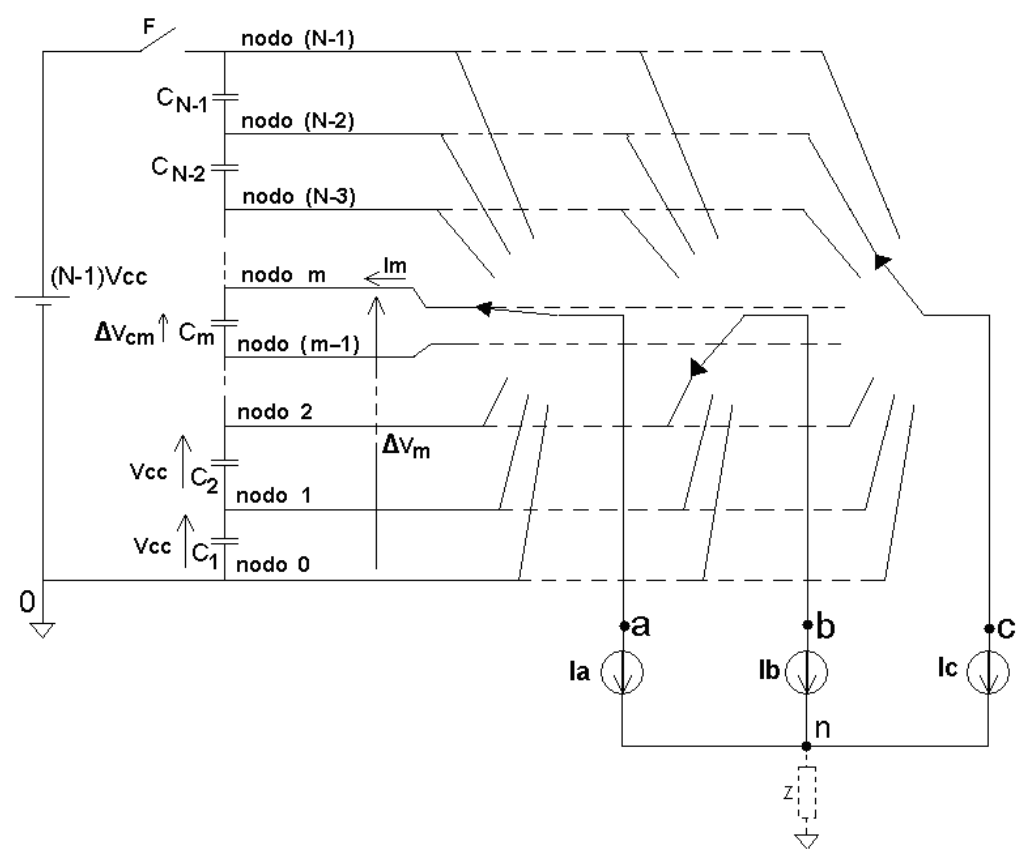

Figura 2.20. Modelo funcional de un inversor DCMC trifásico de $N$ niveles.

Existen diferentes combinaciones de llaves que producen tensiones de línea idénticas. La elección inadecuada de dichas combinaciones lleva al desequilibrio de las tensiones de los capacitores y conduce a la pérdida de niveles. Luego, la síntesis de la tensión de línea debe ser llevada a cabo mediante la elección correcta de dichas combinaciones a fin de mantener el balance de tensión sobre el bus. Entonces, es necesario elaborar un método para predecir las variaciones de tensión sobre cada capacitor del bus y realizar la elección de la combinación óptima. En esta sección se presenta una propuesta para el cálculo de dichas desviaciones, donde se consideran las siguientes hipótesis:

- Todos los capacitores poseen el mismo valor $\left(C_{1}=C_{2}=\ldots=C_{N-I}=C\right)$

- La carga se modela como una fuente trifásica trifilar de corriente sinusoidal balanceada

Para conservar la generalidad, se realiza el análisis teniendo en cuenta la existencia o no de la fuente de continua mediante dos modelos diferentes. Esto se simboliza mediante la 
posición de la llave $F$ (Fig. 2.20). Primero se considera el caso en que la fuente de tensión se encuentra conectada $(F=1)$ y una corriente genérica $I_{m}$ fluye hacia el nodo $m$ como se muestra en la Fig. 2.20. Con referencia a dicha figura, puede observarse que la corriente circula a través de una capacidad equivalente $C_{e q_{-} m}$ dada por:

$$
C_{e q_{-} m}=C_{e q_{-} 1}+C_{e q_{-} 2}=\frac{C}{m}+\frac{C}{(N-1-m)}
$$

donde $C_{e q_{-} l}$ y $C_{e q_{-} 2}$ son las capacidades equivalentes del nodo $m$ respecto a los nodos 0 y $(N-1)$ respectivamente. La variación de tensión en el nodo $m$, respecto al nodo 0 , en un intervalo de tiempo $d T$ es:

$$
\Delta V_{\mathrm{m}}=\frac{1}{C_{\text {eq } \mathrm{m}}} \int_{t_{0}}^{t_{0}+d T} I_{\mathrm{m}}(t) d t
$$

Si la variación de la corriente durante el intervalo $d T$ es pequeña, entonces la variación de tensión puede simplificarse a:

$$
\Delta V_{m}\left\{\begin{array}{l}
=0 \quad \text { si } \quad m=0 \text { o } m=(N-1) \\
\cong \frac{d T I_{m}\left(t_{0}\right)}{C_{e q_{-} m}}=\frac{d T I_{m}\left(t_{0}\right)}{\frac{C}{m}+\frac{C}{(N-1-m)}} \quad 1 \leq m \leq N-2
\end{array}\right.
$$

La variación de tensión sobre cada capacitor depende de su posición relativa al nodo $m$. El capacitor $C_{j}(j=1, . . N-1)$ sufrirá un cambio de tensión $\Delta V_{C j}$ dado por:

$$
\Delta V_{C j}= \begin{cases}\frac{\Delta V_{m}}{m} & 1 \leq j \leq m \\ \frac{-\Delta V_{m}}{(N-1-m)} & m+1 \leq j \leq N-1\end{cases}
$$

En el segundo caso, cuando no existe fuente de tensión conectada al bus $(F=0)$, la capacidad equivalente es $C / m$ dado que no existe camino de circulación de corriente entre el nodo $(N-1)$ y el nodo 0 . La expresión de la variación de tensión sobre el nodo $m$ resulta: 


$$
\Delta V_{m}\left\{\begin{array}{l}
=0 \text { si } \quad m=0 \\
\cong \frac{d T I_{m}\left(t_{0}\right)}{\frac{C}{m}}
\end{array} \quad 1 \leq m \leq N-1\right.
$$

Finalmente, la variación de tensión sobre el capacitor $j$ resulta en este caso:

$$
\Delta V_{C j}=\left\{\begin{array}{cc}
\frac{\Delta V_{m}}{m} & 1 \leq j \leq m \\
0 & m+1 \leq j \leq N-1
\end{array}\right.
$$

Una forma general para la expresión de la variación de tensión del capacitor $C_{j}$, teniendo en cuenta la presencia o ausencia de la fuente de tensión continua puede ser resumida como:

$$
\Delta V_{C j} \cong\left\{\begin{array}{lc}
\frac{1}{m}\left(\frac{d T I_{m}\left(t_{0}\right)}{\frac{C}{m}+\frac{C F}{(N-1-m)}}\right) & 1 \leq j \leq m \\
\frac{F}{(N-1-m)}\left(\frac{-d T I_{m}\left(t_{0}\right)}{\frac{C}{m}+\frac{C}{(N-1-m)}}\right) & m+1 \leq j \leq N-1
\end{array}\right.
$$

$\mathrm{F}=1$ : fuente de tensión presente, $\mathrm{F}=0$ : fuente de tensión ausente

Las variaciones de tensión sobre cada capacitor se calculan sumando las contribuciones de las tres corrientes de salida aplicando el principio de superposición. Esto es, reemplazando $I_{m}$ por $I_{a}, I_{b}$, e $I_{c}$, con el valor de $m$ correspondiente. Hay que mencionar aquí la presencia de la impedancia $\mathrm{Z}$ que se incluye a los efectos de proporcionar un camino ficticio de retorno a cada una de dichas corrientes en forma individual. Debido a que la terna de corrientes es balanceada, dicha impedancia no tiene efecto neto sobre el cálculo de tensiones en el circuito, pues la corriente que circula por ella es cero en todo momento. La variación de tensión en el capacitor $j$ debido a las tres fases resulta:

$$
\Delta V_{C j 3 \phi}=\Delta V_{C j a}+\Delta V_{C j b}+\Delta V_{C j c} \quad 1 \leq j \leq N-1
$$


La expresión (2.12) permite hallar la variación de tensión sobre todos los capacitores del bus para cualquier combinación de tensiones de columna del convertidor. Luego, dada la terna de tensiones de línea y el ciclo de trabajo se evalúan las variaciones de tensión sobre cada capacitor para todas las combinaciones de columna que sintetizan las tensiones de línea deseadas.

De acuerdo con (2.6) la síntesis del vector de referencia se efectúa mediante la aplicación de los vectores $\mathbf{V}_{u l}, \mathbf{V}_{l u} \mathrm{y} \mathbf{V}_{3}$ con sus correspondientes ciclos de trabajo $d_{u l}, d_{l u}$ y $d_{3}$, dentro del ciclo de promediación $T_{S}$. Es decir, que a lo largo de $T_{S}$ las tensiones en los capacitores se modifican debido a la aplicación de cada uno de dichos vectores, según (2.13).

$$
\Delta V_{C j T_{S}}=\Delta V_{C j(u l)}+\Delta V_{C j(l u)}+\Delta V_{C j(3)} \quad j=1, \ldots, N-1
$$

donde, $\Delta V_{C j(u l)}$ es la variación de tensión calculada con (2.12) debido a la combinación de conmutación $\left(m_{a}, m_{b}, m_{c}\right)$, que sintetiza el vector $\mathbf{V}_{u l}$, es decir para las corrientes de fase entrando en los nodos $m_{a}, m_{b}, y \quad m_{c}$, por un intervalo de tiempo igual a $d_{u l} T_{S}$. Lo mismo es válido para $\Delta V_{C j(l u)} y \Delta V_{C j(3)}$. Dado que cada vector admite una o varias posiciones de los interruptores para su síntesis, existen varias secuencias de conmutación posibles. Para cada secuencia de conmutación se obtiene un conjunto distinto de $N-1$ valores de desviación de tensión sobre los capacitores. Luego, la elección adecuada de la secuencia de conmutación puede actuar sobre las tensiones de los capacitores para preservar el equilibrio.

\subsubsection{Procedimiento de optimización}

La búsqueda de la secuencia óptima comienza con la determinación de todas las secuencias posibles para la síntesis de la terna $\mathbf{V}_{u l}-\mathbf{V}_{l u}-\mathbf{V}_{3}$, pero considerando sólo aquellas que producen saltos de tensión de columna entre niveles adyacentes. Esto es necesario para garantizar iguales tensiones de bloqueo sobre los dispositivos de conmutación, como se vio en la Sección 2.3. 
El estado del bus de continua se representa mediante un espacio definido por las tensiones de los capacitores. Entonces, las tensiones de referencia del bus pueden expresarse mediante el vector $\mathbf{V}_{\text {Cref }}$ definido en (2.14):

$$
\mathbf{V}_{\text {Cref }}=\left[\begin{array}{llll}
V_{\text {Cref }} & V_{\text {Cref }} & \ldots . . & V_{\text {Cref }}
\end{array}\right]^{T} \quad, \quad V_{\text {Cref }}=\frac{\sum_{i=1}^{N-1} V_{C i}}{N-1}
$$

En un instante de muestreo $k T_{S}$, el vector de tensiones es:

$$
\mathbf{V}_{C}\left(k T_{S}\right)=\left[\begin{array}{lllll}
V_{C_{N-1}}\left(k T_{S}\right) & V_{C_{N-2}}\left(k T_{S}\right) \quad \ldots \ldots . & V_{C_{1}}\left(k T_{S}\right)
\end{array}\right]^{T}
$$

El valor de tensión en el instante de muestreo $(k+1) T_{S}$ puede expresarse en términos de la muestra anterior y el cálculo de la variación de tensión realizado con (2.13),

$$
\mathbf{V}_{C}\left((k+1) T_{S}\right)=\mathbf{V}_{C}\left(k T_{S}\right)+\Delta \mathbf{V}_{C}\left((k+1) T_{S}\right) \quad \text { donde: } \quad \Delta \mathbf{V}_{C}\left((k+1) T_{S}\right)=\left[\begin{array}{c}
\Delta V_{C(N-1)}\left((k+1) T_{S}\right) \\
\Delta V_{C(N-2)}\left((k+1) T_{S}\right) \\
\cdot \\
\cdot \\
\Delta V_{C 1}\left((k+1) T_{S}\right)
\end{array}\right]
$$

Por otro lado, el vector $\mathbf{V}_{C}\left(k T_{S}\right)$ en términos del error $\mathbf{E}$, es:

$$
\mathbf{V}_{C}\left(k T_{S}\right)=\mathbf{V}_{\text {Cref }}-\mathbf{E}\left(k T_{S}\right)
$$

Entonces, el error en el instante $(k+1)$ resulta:

$$
\mathbf{E}[k+1]=\mathbf{V}_{C r e f}-\mathbf{V}_{C}[k+1]=\mathbf{E}[k]-\Delta \mathbf{V}_{C}[k+1]
$$

Esta expresión indica la necesidad de elegir una secuencia de conmutación que genere un vector $\Delta \mathbf{V}_{C}[k+1]$ en oposición a $\mathbf{E}[k]$. Esto contribuye a que el vector de tensiones muestreadas tienda al vector de referencia.

El proceso de selección de la secuencia se basa en la evaluación de todas las secuencias mediante una función de costo que mida la diferencia entre $\mathbf{V}_{C r e f}$ y $\mathbf{V}_{C}$. Esto puede hacerse evaluando la norma de $\mathbf{E}[k+1]$ : 
$\|\mathbf{E}[k+1]\|_{1}=\left|E_{C_{1}}[k]-\Delta V_{C_{1}}[k+1]\right|+\left|E_{C_{2}}[k]-\Delta V_{C_{2}}[k+1]\right|+\ldots .+\left|E_{C_{N-1}}[k]-\Delta V_{C_{N-1}}[k+1]\right|$

La secuencia de conmutación que minimiza (2.16) es elegida para sintetizar la terna $\mathbf{V}_{u l^{-}}$ $\mathbf{V}_{l u}-\mathbf{V}_{3}$, donde $\Delta V_{C j}(j=1, \ldots, N-1)$ son calculados con (2.13). La Fig. 2.21 resume el diagrama de flujo de todo el proceso.

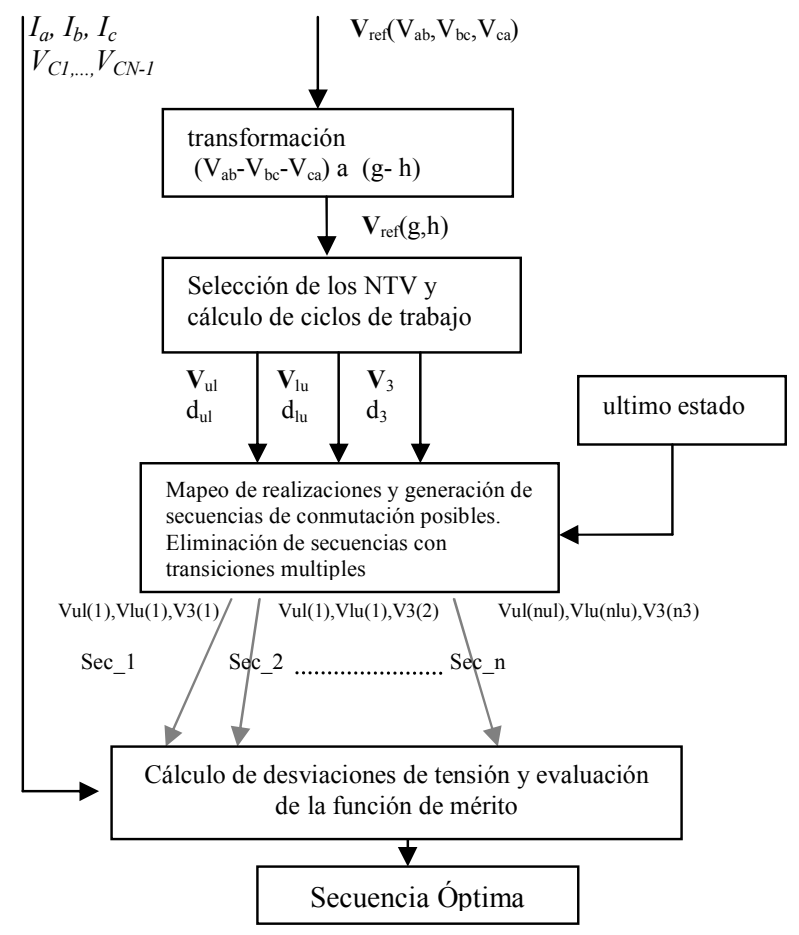

Figura 2.21. Diagrama de optimización.

\subsubsection{Región de estabilidad de la estrategia de balance}

Con referencia a la estabilidad del balance de la tensión de los capacitores del bus $D C$ en convertidores $D C M C$ se ha establecido y verificado que dicho balance posee una región estable restringida para el caso de convertidores con frente pasivo y número de niveles mayor a tres [99][100][101]. Se ha demostrado que la operación del convertidor con alto índice de modulación simultáneamente con alto factor de potencia converge al desbalance del bus $D C$ independientemente del sentido de flujo de potencia y del 
método de modulación. En particular, se ha demostrado en [99] para $N>3$ que el equilibrio del bus puede lograrse si:

$$
f_{s t}(m, \varphi)=m \cdot \cos (\varphi)<0,55
$$

donde $m$ es el índice de modulación y $\varphi$ la fase entre la tensión y la corriente en el lado de alterna del convertidor. La Fig. 2.22 caracteriza la región inestable en función de $m$ y $\varphi$ para el algoritmo de balance aplicado a un convertidor de 5 niveles, resultado que coincide plenamente con (2.17) y los resultados de [99][100] y [101].

En la Fig. 2.23 se muestra el perfil típico de la evolución de las tensiones sobre los nodos del bus medidos con respecto al negativo del bus $D C$ para ambos sentidos de flujo de potencia.

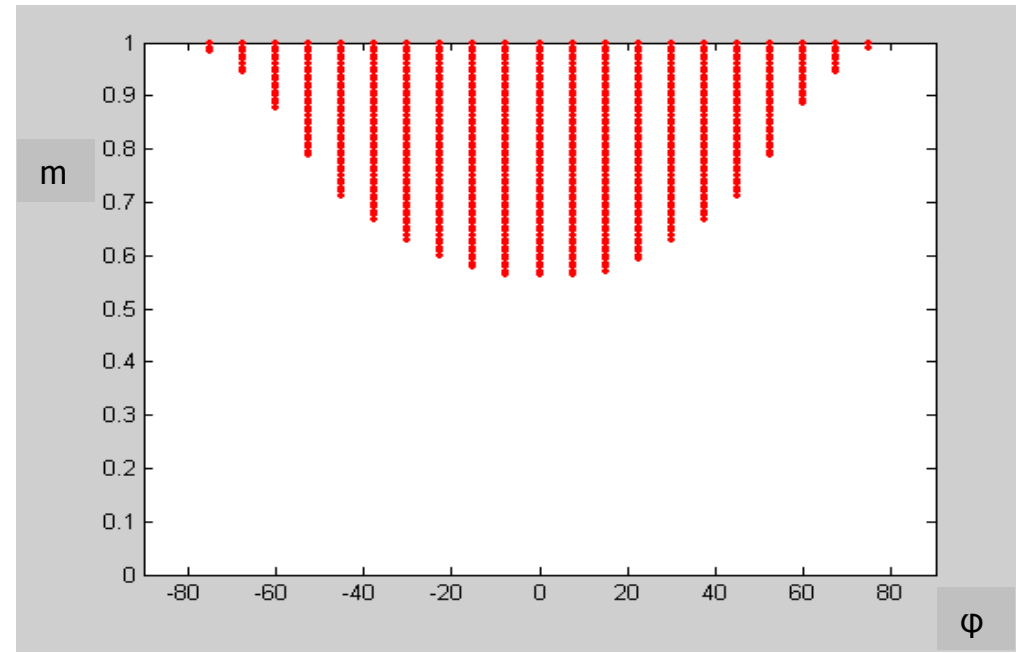

Figura 2.22. Región de inestabilidad de balance del bus DC para el convertidor DCMC con $N=5$. 


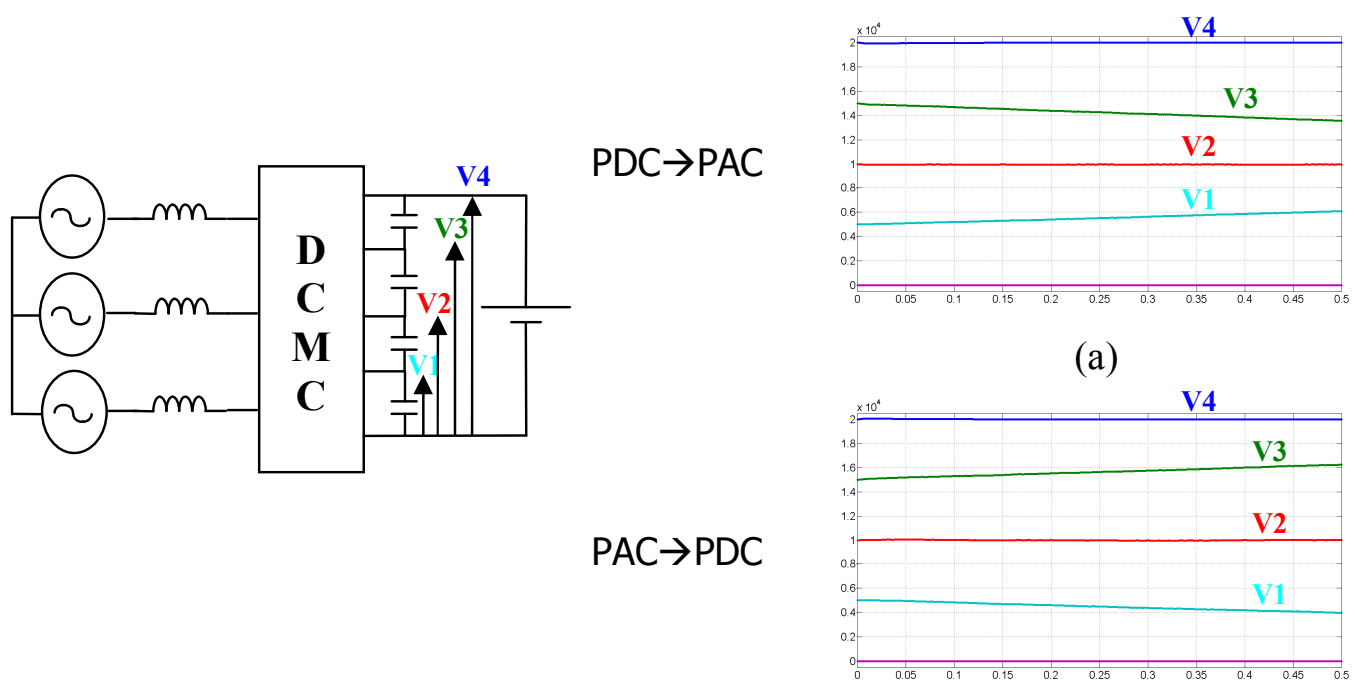

(b)

Figura 2.23. Efecto de desbalance del bus DC para ambos sentidos del flujo de potencia con $f_{\text {st }}>0,55$.

Si bien la región de estabilidad puede ser extendida mediante la utilización de vectores no cercanos, este esquema aumenta las pérdidas de conmutación y la distorsión de la forma de onda sintetizada [102][103][104]. Además, el método no asegura transiciones unitarias en la tensión de salida, lo que puede comprometer la integridad de los dispositivos [93][105].

También es necesario mencionar que los términos establecidos en (2.17) son generales y no dependen del esquema de conmutación de las columnas. Es por ello que esta condición (2.17) resulta ser necesaria pero no suficiente para mantener dicho balance.

\subsection{Aplicación a un compensador de potencia reactiva}

Las validación de los algoritmos de control propuestos se realiza en entorno Simulink. Los mismos se programaron en bloques embebidos de ejecución en tiempo discreto y se incorporó el modelo de un convertidor $D C M C$ de 5 niveles realizado a partir de componentes individuales existentes en la librería SimPowerSystems Blockset. El convertidor y su modulador se aplican en un esquema de compensación de potencia reactiva (DSTATCOM) de una carga inductiva de bajo factor de potencia. 
El compensador estático consiste en una terna trifásica de tensiones sincrónica con la red conectada en paralelo con la carga a compensar mediante una reactancia de acoplamiento. El objetivo del compensador consiste en inyectar la componente de corriente reactiva consumida por la carga en el nodo de conexión, tal que la red de suministro provea sólo corriente activa,

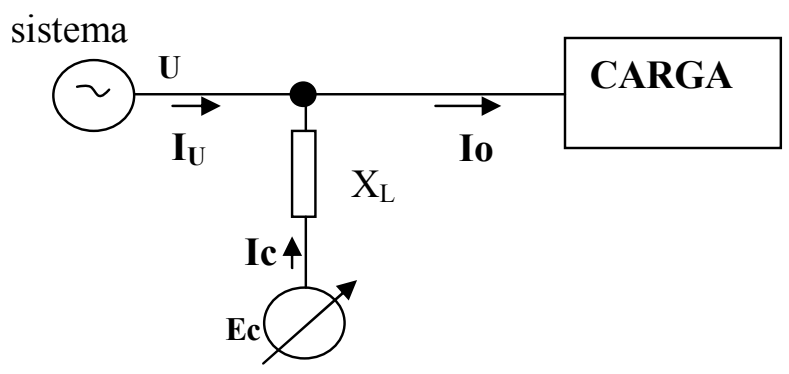

Figura 2.28. Esquema de conexión de un compensador estático.

La inyección de potencia reactiva fundamental en la red se logra mediante la variación de amplitud de la tensión Ec del DSTATCOM. Por la reactancia de acoplamiento $X_{L}$ fluye una corriente Ic, cuya amplitud y fase dependen de la diferencia entre $\mathbf{U}$ y Ec. Si suponemos que la fase entre estos sistemas es cero, entonces circulará por $X_{L}$ una corriente con componente reactiva pura, según se observa en la Fig. 2.29:

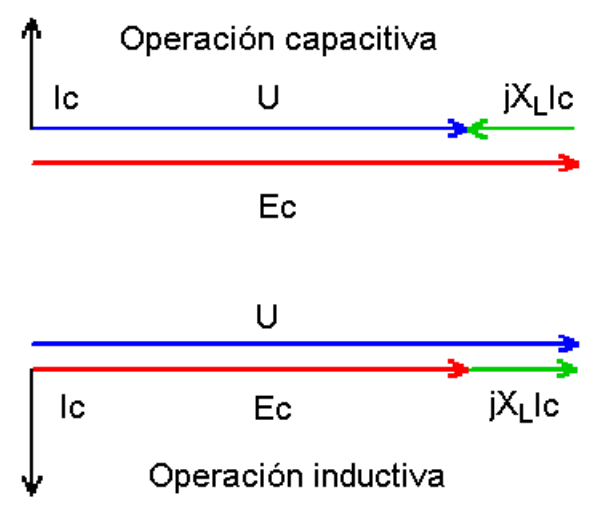

Figura 2.29. Análisis fasorial de un DSTATCOM inyectando y drenando corriente reactiva de la red de suministro.

El sistema de control del DSTATCOM regula la tensión de salida del convertidor para entregar a la red la cantidad necesaria de corriente reactiva. La tensión Ec es colineal 
con $\mathbf{U}$ a diferencia de un pequeño ángulo que permite compensar las pérdidas del convertidor. Si $E c=U$, entonces no circula corriente. Si $E c$ es superior a $U$, entonces la corriente Ic estará adelantada y en cuadratura con la tensión del sistema. En este caso el DSTATCOM se presenta a la red como un capacitor, entregando corriente en adelanto.

El caso de estudio que se presenta consiste en la aplicación de los algoritmos a un convertidor de 5 niveles utilizado como compensador de reactivo en una red de MT. La Fig. 2.30 muestra el diagrama del sistema completo incluyendo carga, convertidor, algoritmo de modulación y controlador de corriente.

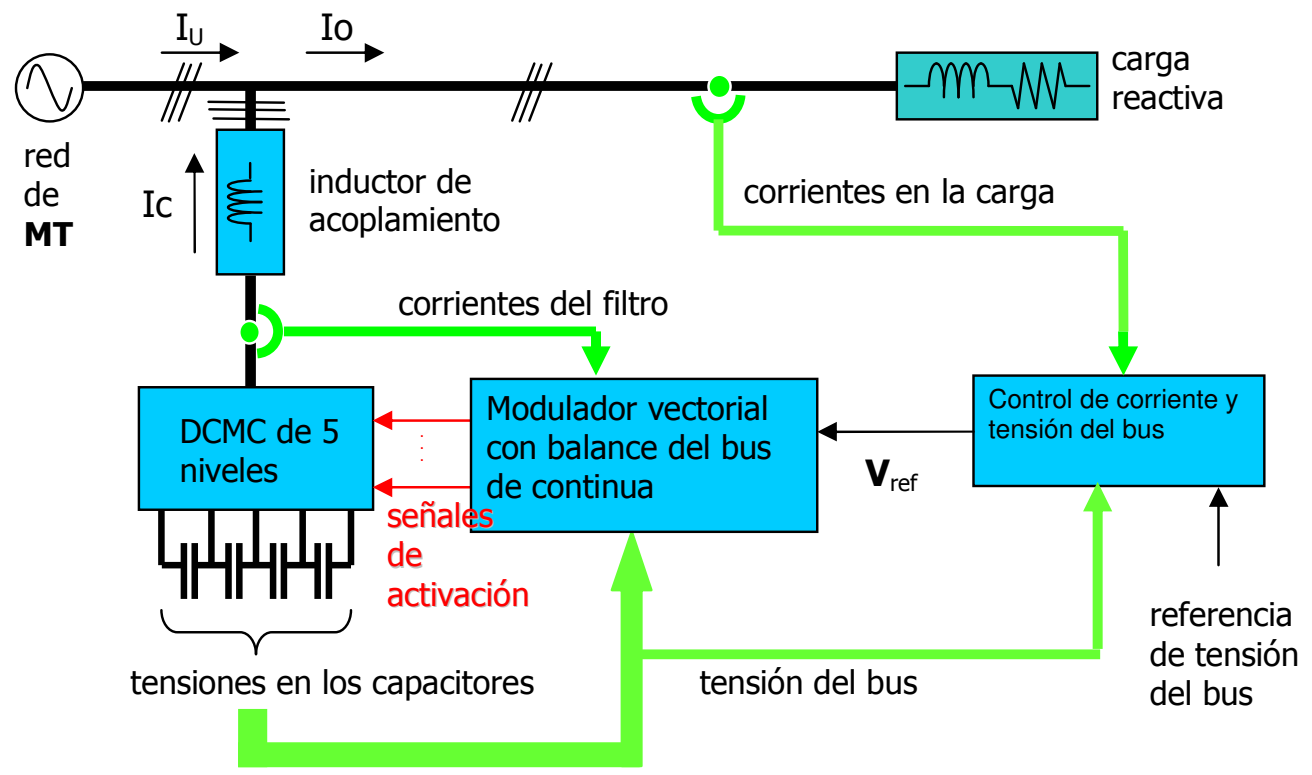

Figura 2.30. Diagrama esquemático del sistema.

El objetivo del sistema es la compensación de corriente reactiva debida a una carga altamente inductiva, a fin de que la red provea sólo la componente activa de la corriente. El bloque de control de corriente (Fig. 2.30) trabaja en el marco de referencia sincrónico con la tensión de red y determina el vector de referencia $\mathbf{V}_{\text {ref }}$ para inyectar la corriente en cuadratura que compense el reactivo de la carga y una componente en fase que compense las pérdidas y estabilice la tensión del bus en el valor nominal.

El primer ensayo consistió en iniciar el sistema en condiciones no compensadas (control de corriente deshabilitado) y habilitarlo en $t=100 \mathrm{~ms}$. La Fig. 2.31 muestra una tensión de fase del sistema y su corriente de línea donde se puede apreciar el bajo factor de potencia de la carga. Durante este lapso el convertidor sigue al sistema $(E c=U)$ y no se 
introduce corriente, excepto la pequeña componente activa necesaria para compensar las pérdidas. En $t=100 \mathrm{~ms}$ se habilita el control y el convertidor aporta la componente reactiva que consume la carga de modo que la red entrega la componente activa. La Fig. 2.32(a) muestra las corrientes aportadas por el convertidor antes y después del instante de activación de la compensación, en tanto que la Fig. 2.32(b) muestra una tensión de línea en bornes del convertidor. La misma luce suave variando entre niveles sucesivos.

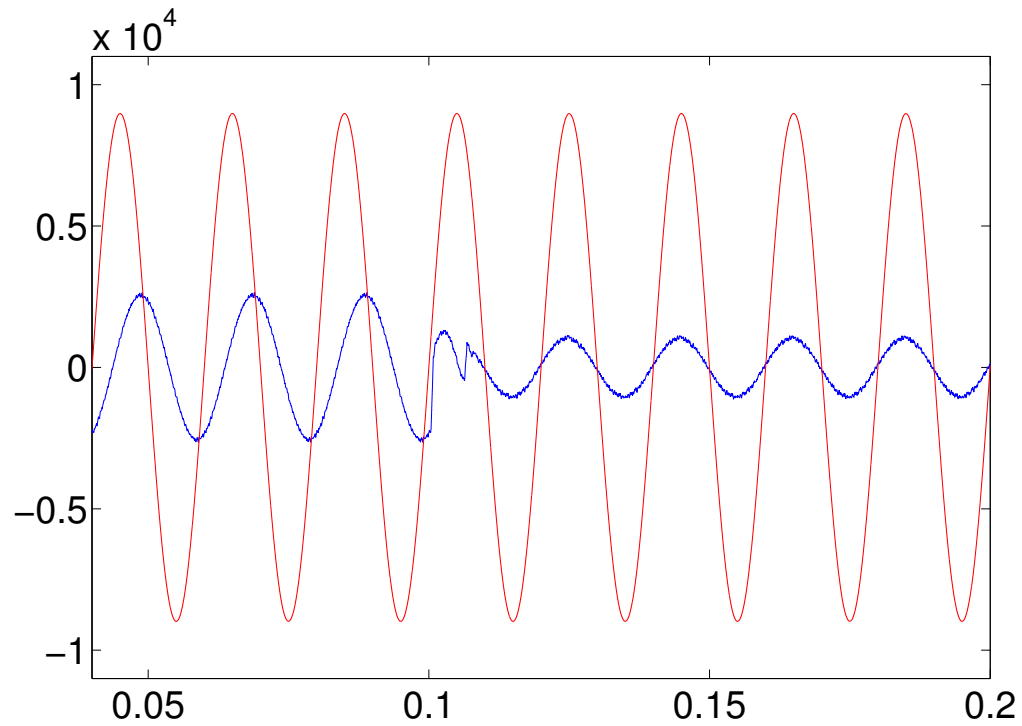

Figura 2.31. Tensión y corriente de una fase de la red de suministro.
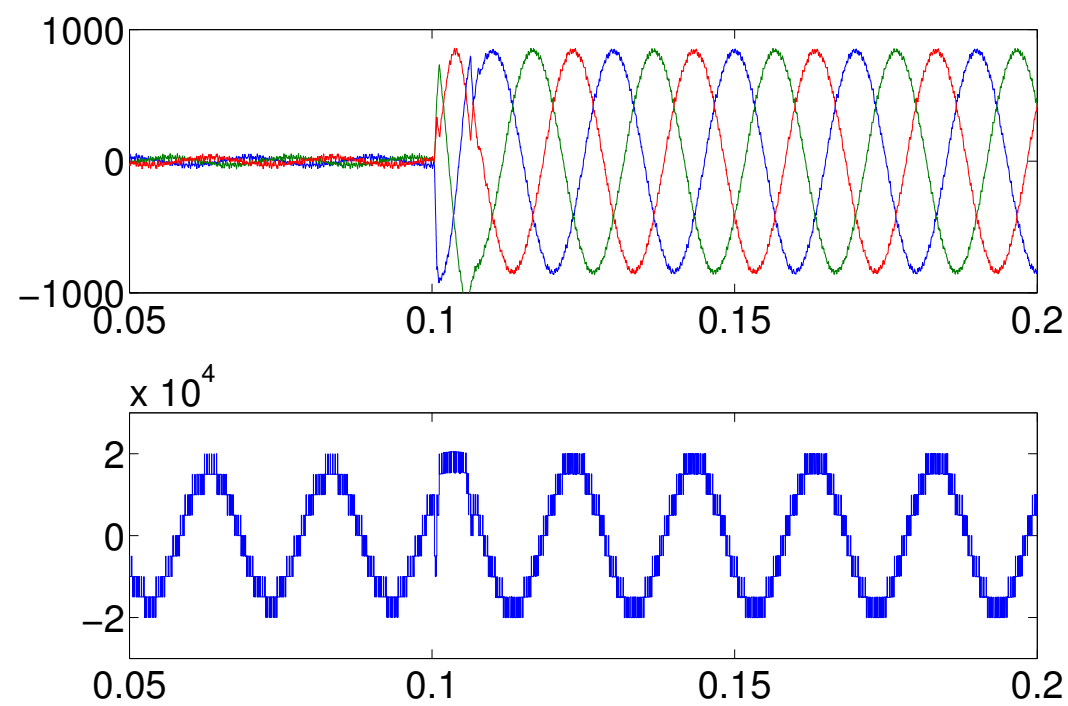

Figura 2.32. (a) Corrientes inyectadas por el convertidor (b) Tensión de una línea del convertidor. 
En la Fig. 2.33 se muestran las tensiones de los capacitores y la tensión del bus de continua. Se observa que los mismos permanecen balanceados aún en el instante de la activación del control, presentando un incremento en este instante debido a la dinámica del control de tensión del bus.

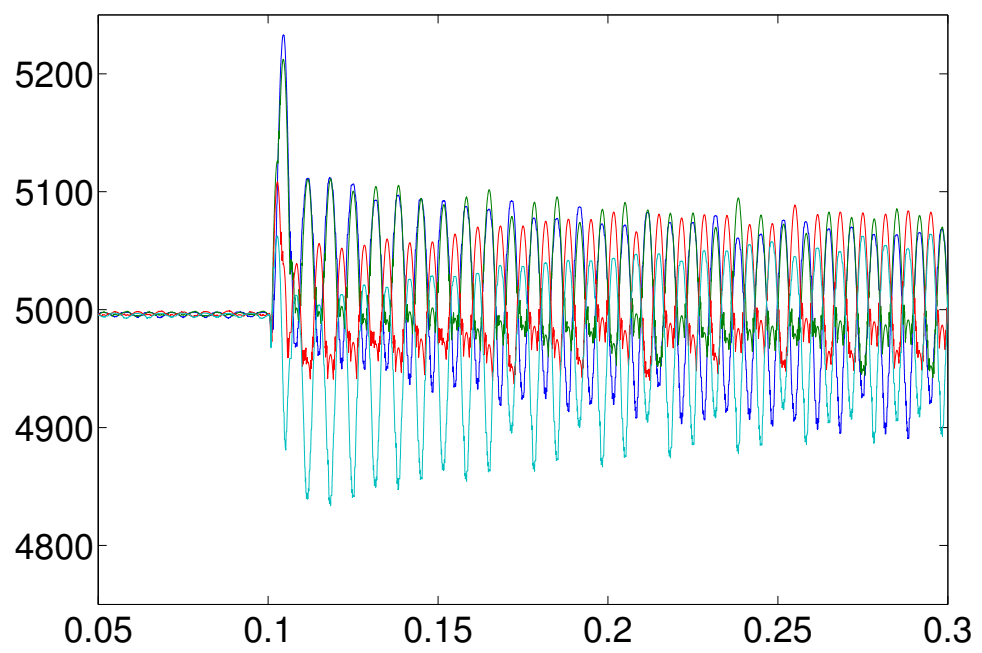

Figura 2.33. Tensiones en los capacitores del bus de continua.

En un segundo ensayo se forzó por medios externos un desbalance en el bus $D C$ en $t=100 \mathrm{~ms}$ (Fig. 2.34), con el control de corriente activado. Aquí se utilizó un índice de modulacion $\mathrm{m}=0$,85. Las tensiones de los capacitores se muestran en la Fig. 2.34. Se observa que la perturbación sobre el balance es mitigada y la condición de equilibrio se restablece aproximadamente en 100ms. En la Fig. 2.35 se observa que incluso en presencia de la perturbación las tensiones de columna se mantienen variando entre niveles sucesivos. 


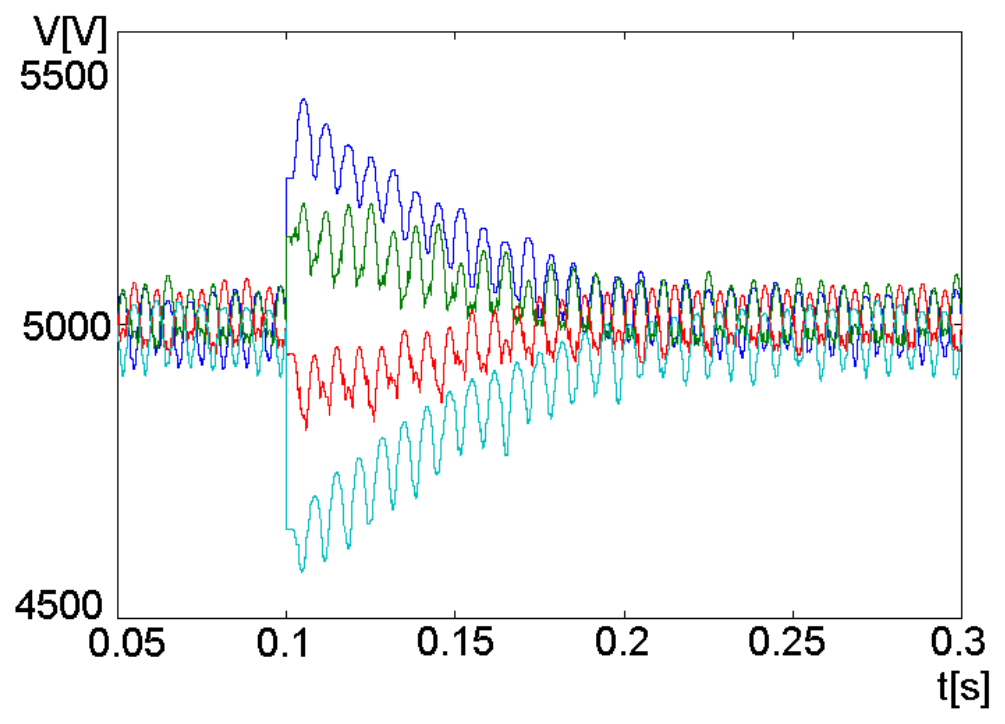

Figura 2.34. Tensiones sobre los capacitores en presencia de desbalance $(m=0,85)$.
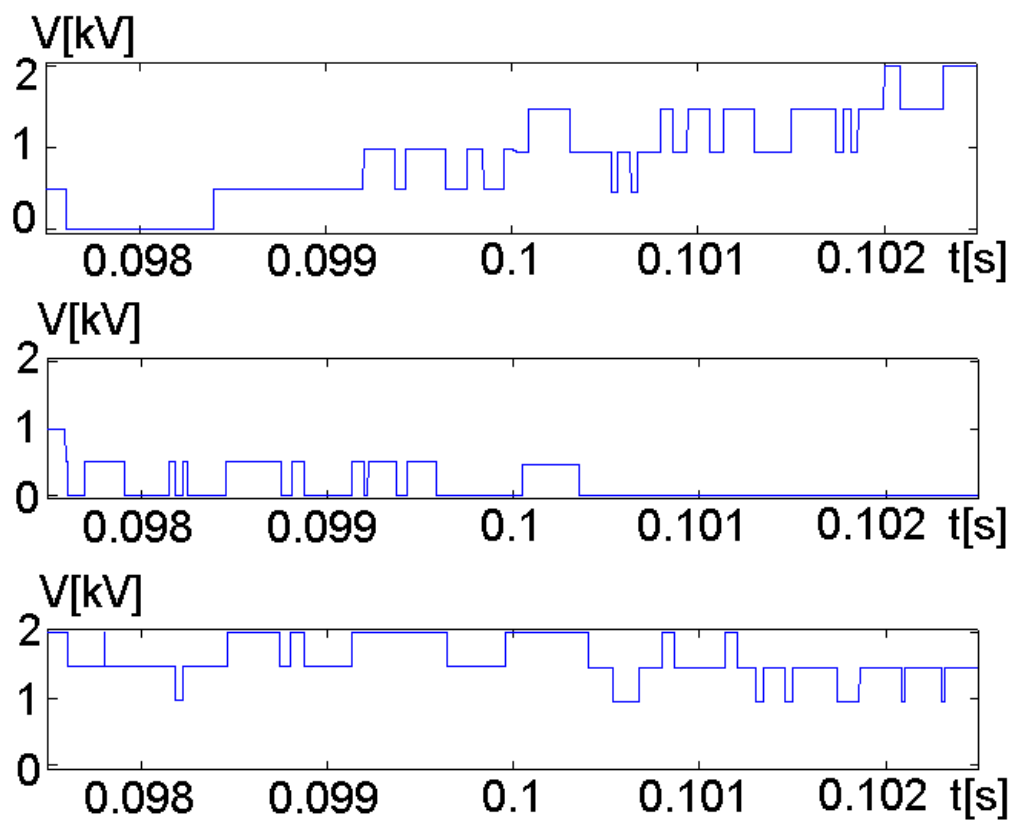

Figura 2.35. Tensiones de columna en el instante de la perturbación de balance $(m=0,85)$.

Finalmente se modificó el índice de modulación a $m=0,96$ y se repitió el ensayo anterior. Se verifica que el tiempo necesario para restablecer el balance se prolonga levemente con respecto al caso anterior (Fig. 2.36). Por otro lado se observa en la tensión de columna (Fig. 2.37) que existen menos conmutaciones entre niveles. Esto tiene sentido teniendo en cuenta que la operación a índices de modulación mayores 
implica menor disponibilidad de estados redundantes, lo que limita la acción sobre el control de desbalance. De esto se deriva además, que no es posible operar el convertidor en sobremodulación ya que en este caso el control de balance perdería efectividad.

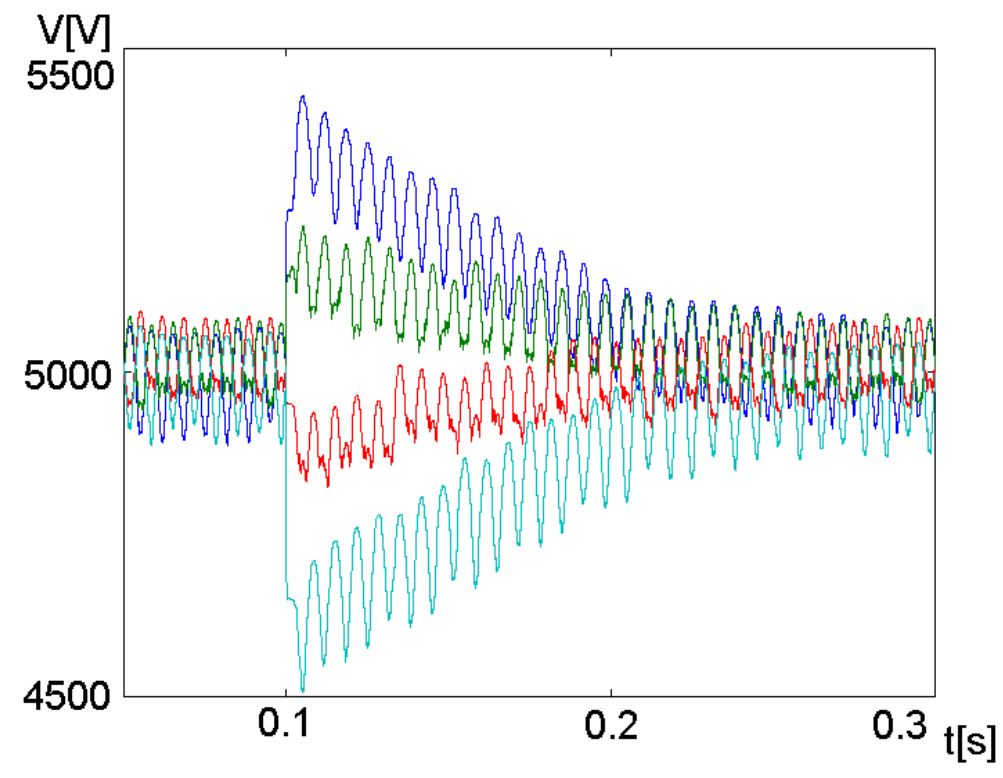

Figura 2.36. Tensiones sobre los capacitores en presencia de desbalance $(m=0,96)$.
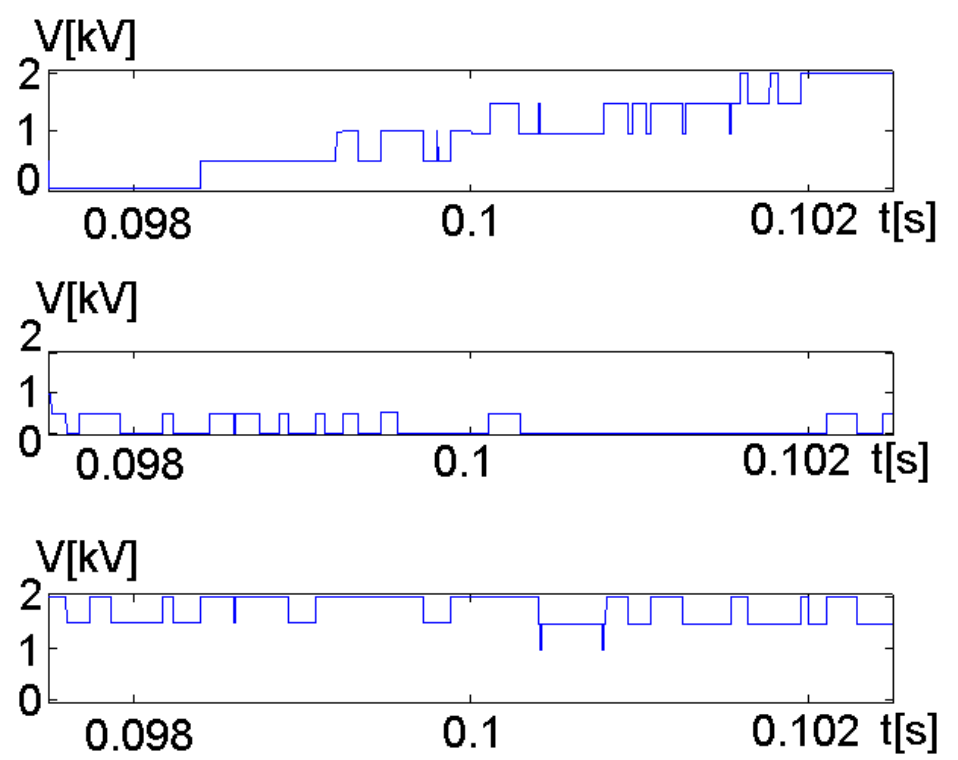

Figura 2.37. Tensiones de columna en el instante de la perturbación de balance $(m=0,96)$. 


\subsection{Conclusiones}

Este capítulo presentó a los convertidores multinivel con fuente de tensión y sus ventajas con respecto a la baja distorsión armónica y a la mayor magnitud de la tensión de salida, la cual se extiende más allá de los dispositivos de conmutación. En especial se realizó un análisis del convertidor multinivel enclavamiento por diodos. Se analizaron las características propias de conmutación de la topología y la característica de redundancia en sus tensiones de línea. Se introdujo el concepto de realización del vector de línea y su efecto sobre las corrientes inyectadas en el bus de continua. Se utilizó un esquema de modulación vectorial y se propuso una estrategia generalizada para lograr el balance del bus de continua. El algoritmo fue evaluado a través de una aplicación de compensación de potencia reactiva en media tensión (DSTATCOM), presentando un comportamiento adecuado y verificando la consistencia de los resultados con los existentes en la literatura en lo que se refiere a la región de estabilidad. 



\section{Capítulo 3}

\section{Control Predictivo en Convertidores}

\subsection{Introducción}

El control de corriente es un tópico de gran importancia en la electrónica de potencia. El principio de volt-segundos equivalentes ha sido desde el principio el mecanismo central del control de los inversores. La estrategia clásica consiste en un esquema de control lineal de las corrientes implementado en un lazo externo al convertidor cuya tensión de salida es modulada mediante ancho de pulso o en forma vectorial como se vió en el capítulo anterior. En este caso el convertidor se considera en su versión promediada como un amplificador de tensión, requiriendo una frecuencia de conmutación suficientemente alta para no interferir en el lazo externo de control de corriente. Esta característica limita el ancho de banda del sistema y restringe sus posibilidades en aplicaciones de alto requerimiento dinámico y baja frecuencia de conmutación como por ejemplo filtrado de armónicos.

La técnica de control predictivo basado en modelo (MPC: model-based predictive control) aplicada a convertidores electrónicos en su variante de estados finitos (FCSMPC: finite control-set model predictive control) no requiere moduladores pues incorpora la característica discreta de los convertidores de potencia. Este enfoque permite atenuar la relación de compromiso entre frecuencia de conmutación y ancho de banda a la vez que proporciona gran flexibilidad para el control multivariable. La estrategia del $M P C$ es similar a la desarrollada para el control de balance del bus $D C$ del capítulo anterior. Es decir, se trata de precalcular los valores de corriente de salida en base a un modelo dinámico del sistema y elegir la mejor combinación de conmutación para guiar dichas variables hacia sus valores de referencia. La combinación de ambos 
esquemas resulta consistente y unifica ambos controles en una misma estrategia de control para el $D C M C$.

En este capítulo se desarrolla un control conjunto de las corrientes y del balance del bus de continua del DCMC utilizando la técnica $F C S-M P C$. El esquema adoptado permite controlar en forma directa las variables de interés prescindiendo de moduladores y controladores adicionales e incorporando en forma explícita las restricciones de conmutación propias de la topología. De esta manera, el método unifica la estructura de control de las variables internas y externas del convertidor proporcionando consistencia a la solución y permite reducir la cantidad de estados a evaluar por el algoritmo predictivo. Se muestran resultados sobre la aplicación de un convertidor $D C M C$ de 5 niveles como filtro activo de armónicas conectado a la red de media tensión. Los aportes originales con relación al contenido del capítulo se presentan en [64][65] y [66].

\subsection{Nociones de control predictivo}

El nombre genérico "control predictivo" engloba aquellas estrategias de control basadas en el cálculo anticipado del estado del sistema en base a un modelo dinámico explícito del mismo. La idea surgió inicialmente en el ámbito de control de procesos relacionados con la industria del petróleo y también en plantas de generación eléctrica [106][107]. Aquellos conceptos tuvieron gran éxito en el ambiente industrial donde posteriormente surgieron variantes de las ideas originales a lo largo de las décadas del 1970 y 1980. El desarrollo de la técnica surgió de manera peculiar, ya que fue el sector industrial el que en mayor medida impulsó su estudio e implementación, llegando posteriormente al ámbito académico, donde se apuntalaron las bases y se proporcionó rigurosidad a las formulaciones. En el inicio, la técnica fue instrumentada sobre plantas con dinámicas lentas, resultando de especial eficacia para el control de sistemas multivariables con restricciones y grandes retardos. Sin embargo, gracias al incremento de velocidad de las plataformas computacionales, la técnica ha ganado popularidad en diversos nichos tecnológicos como la mecatrónica, la industria automotriz y los convertidores electrónicos [44][108]. 
Todas las variantes de control predictivo se encuadran dentro del tipo de controladores óptimos. El núcleo del algoritmo se basa en precalcular el estado y las salidas de la planta para el conjunto admitido de secuencias de control a lo largo del horizonte de predicción de largo $N_{h}$ muestras. En términos generales, dado un estado inicial de la misma (medido o estimado) y una secuencia de control $\mathbf{u}$, la evolución temporal del sistema puede ser calculada y comparada con la referencia mediante una función de costo que instrumente el criterio de optimización deseado. En general, la trayectoria del estado al instante $\left(k+N_{h}\right)$ puede ser calculada en el instante $k$ usando un modelo dinámico del mismo, el estado actual y la secuencia de actuación a lo largo del horizonte de predicción. Formalmente el algoritmo consta de los siguientes elementos fundamentales [109]:

1) Modelo de predicción. Se utiliza de forma explícita para calcular la salida del sistema en instantes de tiempo futuros.

2) Función de costo. Representa el índice objetivo que se desea optimizar.

3) Restricciones operativas. Son las regiones permitidas tanto para los estados como para las salidas del sistema. Aquí pueden estar programadas las saturaciones de actuadores y los valores máximos o mínimos de seguridad de variables críticas.

4) Horizonte de predicción. Es el marco temporal dentro del cual se realiza la optimización. Puede ser fijo o móvil, dando origen a dos enfoques diferentes en la resolución del problema.

\section{El modelo de predicción}

El modelo utilizado debe estar expresado en el dominio del tiempo. El mismo puede ser lineal o no lineal, monovariable o multivariable, en el espacio de estados o mediante función de entrada-salida. Para el caso general de sistemas no lineales, la predicción en el instante $k$ del estado $\mathbf{x}$ en el instante $(k+j+1)$ puede expresarse en términos del estado anterior y la secuencia de actuaciones aplicada a partir del instante $k$ y a lo largo del horizonte de predicción de largo $N_{h}$ muestras mediante (3.1):

$$
\mathbf{x}[k+j+1 \mid k]=f(\mathbf{x}[k+j \mid k], \mathbf{u}[k+j \mid k]) \quad j=0,1, . .,\left(N_{h}-1\right)
$$


donde $\mathbf{u}[k+j \mid k]$ es un componente de una secuencia temporal de vectores de dimensión $p$ (número de entradas):

$$
\mathbf{u}_{F}[k]=\left\{\mathbf{u}[k \mid k], \mathbf{u}[k+1 \mid k], \ldots, \mathbf{u}[k+j \mid k], \ldots, \mathbf{u}\left[k+N_{h}-1 \mid k\right]\right\}
$$

\section{Función de costo}

Constituye el criterio de optimización. Es una función definida positiva que evalúa el costo asociado a la evolución del sistema debido a una secuencia de actuación dada a lo largo del horizonte de predicción.

$$
J_{N_{h}}\left(\mathbf{x}_{k}, \mathbf{u}_{F}[k]\right)=g_{E}+g_{R}=J_{1}\left(\mathbf{x}\left[k+N_{h} \mid k\right]\right)+\sum_{i=0}^{N_{h}-1} J_{2}(\mathbf{x}[k+i \mid k], \mathbf{u}[k+i \mid k])
$$

donde la función $g_{E}$ evalúa el estado final y $g_{R}$ su trayectoria a lo largo del horizonte de predicción. Ambas son definidas positivas y dependen de la secuencia de control (3.2).

\section{Restricciones de operación}

Las restricciones de operación definen los límites dentro de las cuales se prevé la evolución el sistema. Esto permite incluir la saturación de actuadores estableciendo límites a sus señales de comando, como así también imponer límites de seguridad en variables de interés, como aquellas que sean susceptibles de sobrecarga. Las mismas se expresan sintéticamente en (3.4):

$$
\begin{aligned}
& x_{k} \in X^{n}, x_{k}=x[k \mid k] \quad \forall k \\
& u_{k} \in U^{p}, u_{k}=u[k \mid k] \quad \forall k
\end{aligned}
$$

donde $X^{n}$ y $U^{p}$ son los conjuntos de valores admitidos de los estados y de la actuación con dimensiones $n$ (número de estados) y $p$ (número de entradas), respectivamente. También puede incluirse una condición terminal, es decir un conjunto estricto de valores $\Omega^{\mathrm{n}}$ entre los cuales debe estar el valor del sistema al final del horizonte de predicción:

$$
x\left(k+N_{h} \mid k\right) \in \Omega^{n}
$$


El esquema $M P C$ consiste en la resolución de un problema de optimización a lazo abierto dentro del horizonte. Esto es, suponiendo que se conoce la referencia con anticipación, se busca la secuencia de control u para optimizar la trayectoria dentro de la ventana de optimización $N_{h}$ según la función de costo (3.3). Este esquema no es capaz de asimilar y corregir perturbaciones, por lo que suele incorporarse realimentación a través de la variante denominada de horizonte deslizante (RHC: Receding Horizon Control). En este caso el problema de optimización se realiza en cada instante de muestreo pero sólo se aplica la primera muestra de la secuencia óptima elegida $\mathbf{u}[k \mid k]$ desechando los restantes elementos. El nuevo estado del sistema se muestrea y vuelve a buscarse un nuevo vector de entradas u. Este enfoque involucra una gran carga computacional que a su vez depende en forma crítica del largo de horizonte considerado. Éste, en conjunto con el número de entradas y la cantidad de valores que éstas pueden asumir hacen de la optimización un problema computacional complejo y difícil de resolver en tiempo real.

\subsection{Control predictivo en convertidores de potencia}

En [44] se clasifican algunas estrategias clásicas de control de convertidores conmutados que pueden encuadrarse en el esquema general de control predictivo. En dicha clasificación se enumeran las dos variantes $M P C$ que se muestran en la Fig. 3.1. La estrategia de conjunto continuo consiste en realizar el cálculo de optimización considerando el modelo promediado del convertidor y realizando modulación $P W M$ o $S V M$ a frecuencia fija. Un ejemplo de este enfoque puede verse en [110]. Sin embargo, la naturaleza discreta de los convertidores de potencia y las restricciones de conmutación propias de cada topología hacen que el número de estados posibles sea finito. Esto puede incorporarse explícitamente al esquema $M P C$ dando origen a la variante denominada de estados finitos FCS-MPC. A diferencia del esquema basado en el modelo promediado éste permite omitir la etapa de modulación externa, mientras que otro aspecto característico de dicha variante tiene que ver con el horizonte de predicción. El crecimiento exponencial del número de secuencias posibles de u con $N_{h}$,

junto con la alta frecuencia de muestreo y conmutación de los convertidores hacen prohibitiva la extensión del horizonte de predicción a más de dos o tres muestras, considerándose en la mayoría de $\operatorname{los} \operatorname{casos} N_{h}=1$ [44]. En este caso el algoritmo de 
optimización se reduce a la evaluación del estado final mediante la función $g_{E}$ (Ec. (3.3)) y es fácilmente comprensible, pudiéndose resolver en forma viable mediante cálculo directo.

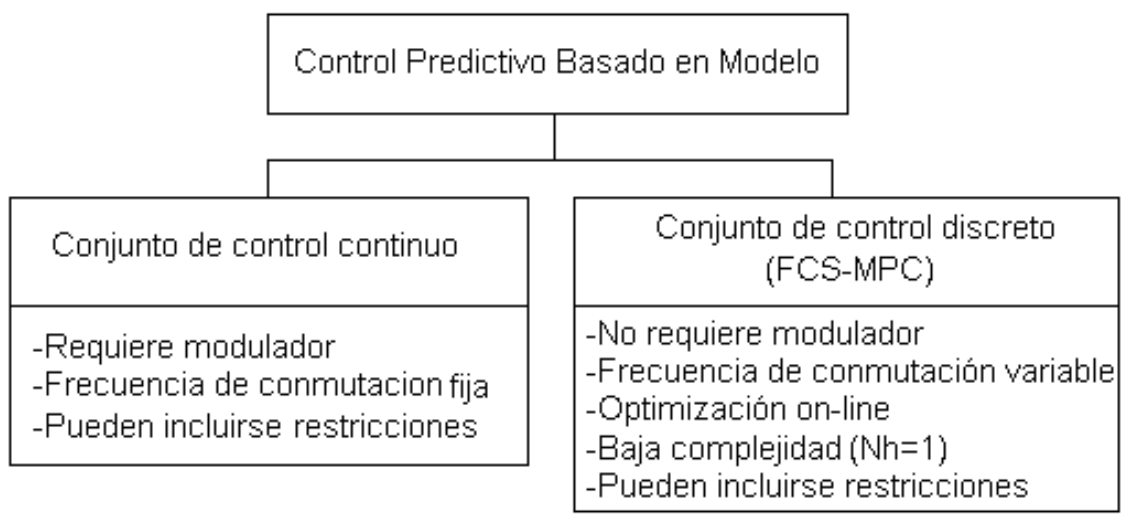

Figura 3.1. Técnicas MPC utilizadas en convertidores electrónicos.

Existen en la literatura varios antecedentes en la utilización de esta técnica incluyendo control de corriente [111], control de potencia [46][47], control de máquinas de inducción [49] y de máquinas sincrónicas [112]. De esta forma, el criterio de optimización evalúa diferentes variables de interés en el sistema, tanto internas (tensión en el bus $D C$, número de conmutaciones) como externas al convertidor (corriente, potencia, par, flujo, etc.) [38]. La técnica también ha sido adoptada para el control de convertidores de tres niveles, constituyendo, en este caso, un enfoque particularmente interesante para el control conjunto de las corrientes y las fluctuaciones de tensión sobre los capacitores [113][114][115][116]. En este sentido, se vio en el Capítulo 2 que la operación del convertidor $D C M C$ con número de niveles mayor a tres requiere ejecutar un control de balance sobre las tensiones de los capacitores (variables internas) incluyendo las restricciones de conmutación propias de la topología. En [117] se realiza el control predictivo de las corrientes y tensiones del convertidor DCMC de 4 niveles utilizando como conjunto de control los $4^{3}=64$ estados del convertidor. Para 5 niveles este número ascendería a $5^{3}=125$. Considerando que en cada periodo de muestreo es necesario calcular las variables de interés (corrientes y tensiones del bus $D C$ ) para todos los estados posibles, es claro que surge un problema en cuanto a la posibilidad de realizar dichos cálculos a las frecuencias usuales de los convertidores electrónicos. En este sentido, es clara la necesidad de buscar mecanismos a fin de aliviar la gran carga computacional involucrada. 
En cuanto a la estabilidad de estas estrategias puede mencionarse que el análisis del problema de optimización para horizonte finito y sistemas continuos está ampliamente estudiado y posee solución cerrada. Sin embargo, la incorporación de restricciones en esquemas con horizonte deslizante y la inclusión explícita de sistemas con dinámica híbrida (como los convertidores electrónicos) introducen serias dificultades analíticas, dejando en este sentido varios problemas abiertos. En [118] y [119] se examinan estos aspectos, mientras que en [120][121] se presenta un resumen del estado del arte al respecto y se introducen lineamientos conceptuales para el análisis de la estabilidad de una topología de convertidor multicelda muy simple.

\subsection{Control predictivo del convertidor DCMC}

El sistema analizado consiste en un convertidor $D C M C$ de $N$ niveles conectado a una red trifásica de media tensión mediante un inductor de acoplamiento $Z_{f}$ como se esquematiza en la Fig. 3.2:

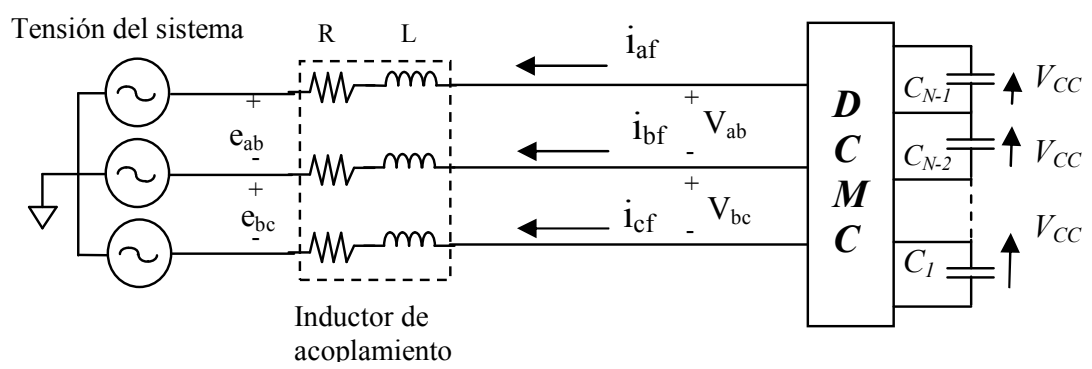

Figura 3.2. Conexión del DCMC a la red mediante inductor de acoplamiento.

Dado que el sistema es trifilar sin neutro, las corrientes del convertidor se determinan a partir de las tensiones de línea $e_{a b}, e_{b c}, V_{a b} \mathrm{y} V_{b c}$, donde las dos últimas pueden asumir sólo valores múltiplos de $V_{C C}$ desde $-(N-1) V_{C C}$ hasta $(N-1) V_{C C}$. Luego pueden representarse las ecuaciones de las corrientes en términos de dos tensiones de línea. Las ecuaciones del circuito son: 


$$
\left\{\begin{array}{l}
V_{a b}-L \frac{d i_{a f}}{d t}-R i_{a f}-e_{a b}+L \frac{d i_{b f}}{d t}+R i_{b f}=0 \\
V_{b c}-L \frac{d i_{b f}}{d t}-R i_{b f}-e_{b c}+L \frac{d i_{c f}}{d t}+R i_{c f}=0 \\
i_{a f}+i_{b f}+i_{c f}=0
\end{array}\right.
$$

El control del convertidor debe satisfacer dos aspectos fundamentales: el seguimiento de las corrientes de salida y por otra parte el balance de tensión en los capacitores del bus de continua. En segundo término pueden considerarse parámetros adicionales como ser frecuencia de conmutación, tensión de modo común entre otras [38].

Como se observa en (3.6), las corrientes del convertidor dependen de la tensión del sistema, de los parámetros del inductor de acoplamiento y de las combinaciones de conmutación que sea capaz de sintetizar el convertidor. En este sentido la cantidad de combinaciones de conmutación de un convertidor DCMC de $N$ niveles resulta $N^{3}$. Entonces, en cada período de muestreo deben calcularse dos corrientes de salida y $(N-1)$ tensiones de capacitores para los $N^{3}$ estados posibles del convertidor. Como se puede apreciar el número de cálculos necesarios es elevado. Sin embargo, si se tiene en cuenta la condición de conmutación que se refiere a la imposibilidad de conmutar en más de un nivel en forma simultánea (Sección 2.3) puede tomarse un subconjunto de estados posibles. El modelo funcional de la Fig. 2.20 se repite para $F=0$ en la Fig. 3.3. Durante un período de conmutación $k$, las columnas del convertidor permanecen conectadas a los nodos del bus $m_{a}, m_{b}, m_{c}\left(m_{a}, m_{b}, m_{c}=0, \ldots, N\right)$ definiendo las tensiones de línea $V_{a b}$, $V_{b c}$ y consecuentemente $V_{c a}$. Debido a la condición mencionada previamente los estados de conmutación para dichas columnas en el periodo posterior de conmutación $(k+1)$ estarán restringidas al subconjunto $U$ descripto por la expresión (3.7), donde $\Delta_{x}$ $(x=a, b, c)$ representa el incremento en el nivel de tensión en cada columna del convertidor referido a la tensión del capacitor $V_{C C}$ : 


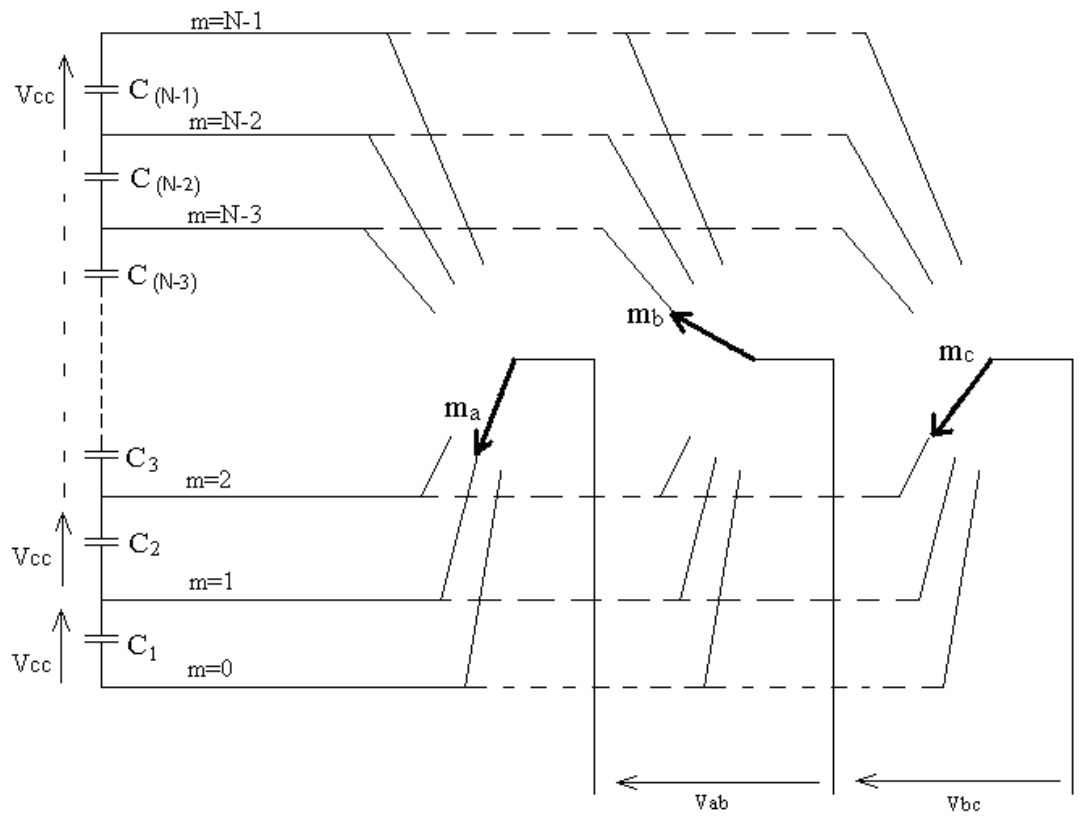

Figura 3.3. Modelo funcional del inversor DCMC de N niveles.

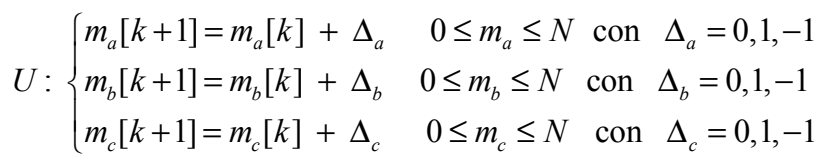

Esta condición relaciona el subconjunto de combinaciones de conmutación posibles con el estado de conmutación previo. Independientemente del número de niveles $N$ del convertidor, dicho subconjunto se limita a un máximo de $3^{3}=27$ estados posibles, lo cual implica un alivio significativo en lo que respecta al número de cálculos, especialmente a medida que $N$ aumenta. Por ejemplo, para un convertidor de 5 niveles el número de combinaciones a evaluar se reduce de $5^{3}=125$, a 27 de acuerdo con el razonamiento anterior.

\subsubsection{Control multi-objetivo}

Para cada combinación de conmutación admitida por (3.7) todas las variables de interés se calculan y se evalúan mediante una función de costo global. Según la composición de la misma y la importancia que cada componente merece dentro del objetivo de control, se pueden elegir distintos factores de peso a fin de enfatizar su importancia en el proceso de optimización [122]. En el caso que se presenta, la función de costo posee tres términos como lo indica la Ec. (3.8): 


$$
g=K_{I} g_{I}+K_{V} g_{V}+K_{n} g_{n}
$$

donde $g_{I}, g_{V}$ y $g_{n}$ son las funciones de costo asociadas al seguimiento de corriente, regulación del balance de tensión sobre los capacitores y reducción de la frecuencia de conmutación y $K_{I}, K_{V}, K_{n}$ son sus correspondientes factores de peso. Esta función se evalúa para las potenciales combinaciones de conmutación definidas en (3.7) (27 como máximo), y aquella que la minimiza es elegida como próximo estado del convertidor.

\subsubsection{Función de costo de corriente}

La ecuación (3.6) permite calcular las variaciones de corriente de un periodo de muestreo al siguiente en términos de las tensiones del sistema y de los parámetros del inductor de acoplamiento. Discretizando las derivadas mediante la aproximación de Euler y considerando un tiempo de muestreo $T_{S}$ :

$$
\frac{d i}{d t} \simeq \frac{i[k+1]-i[k]}{T_{S}}
$$

Y reemplazando (3.9) en (3.6):

$$
\begin{aligned}
& \left\{\begin{array}{l}
V_{a b}[k]-L\left(\frac{i_{a f}[k+1]-i_{a f}[k]}{T_{S}}\right)-R i_{a f}[k]-e_{a b}[k]+L\left(\frac{i_{b f}[k+1]-i_{b f}[k]}{T_{S}}\right)+R i_{b f}[k]=0 \\
V_{b c}[k]-L\left(\frac{i_{b f}[k+1]-i_{b f}[k]}{T_{S}}\right)-R i_{b f}[k]-e_{b c}[k]+L\left(\frac{i_{c f}[k+1]-i_{c f}[k]}{T_{S}}\right)+R i_{c f}[k]=0 \\
i_{c f}[k]=-\left(i_{a f}[k]+i_{b f}[k]\right)
\end{array}\right. \\
& \text { eliminando } i_{c f}: \\
& \left\{\begin{array}{l}
V_{a b}[k]-e_{a b}[k]-L \frac{i_{a f}[k+1]-i_{a f}[k]}{T_{S}}-R i_{a f}[k]+L \frac{i_{b f}[k+1]-i_{b f}[k]}{T_{S}}+R i_{b f}[k]=0 \\
V_{b c}[k]-e_{b c}[k]-L \frac{i_{b f}[k+1]-i_{b f}[k]}{T_{S}}-R i_{b f}[k]-L \frac{i_{b f}[k+1]-i_{b f}[k]}{T_{S}}-L \frac{i_{a f}[k+1]-i_{a f}[k]}{T_{S}}-R i_{a f}[k]-R i_{b f}[k]=0
\end{array}\right.
\end{aligned}
$$




$$
\left\{\begin{array}{l}
2 V_{a b}[k]-2 e_{a b}[k]-2 L \frac{i_{a f}[k+1]-i_{a f}[k]}{T_{S}}-2 R i_{a f}[k]+2 L \frac{i_{b f}[k+1]-i_{b f}[k]}{T_{S}}+2 R i_{b f}[k]=0 \\
V_{b c}[k]-e_{b c}[k]-L \frac{i_{a f}[k+1]-i_{a f}[k]}{T_{S}}-R i_{a f}[k]-2 L \frac{i_{b f}[k+1]-i_{b f}[k]}{T_{S}}-2 R i_{b f}[k]=0
\end{array}\right.
$$

sumando ambas ecuaciones:

$$
\begin{aligned}
& 2 V_{a b}[k]+V_{b c}[k]-2 e_{a b}[k]-e_{b c}[k]-3 L \frac{i_{a f}[k+1]}{T_{S}}+\frac{3 L}{T_{S}} i_{a f}[k]-3 R i_{a f}[k]=0 \\
& 2 V_{a b}[k]+V_{b c}[k]-2 e_{a b}[k]-e_{b c}[k]+\left(\frac{3 L}{T_{S}}-3 R\right) i_{a f}[k]=3 L \frac{i_{a f}[k+1]}{T_{S}} \\
& i_{a f}[k+1]=\frac{T_{S}}{3 L}\left(2 V_{a b}[k]+V_{b c}[k]-2 e_{a b}[k]-e_{b c}[k]\right)+\left(1-\frac{R T_{S}}{L}\right) i_{a f}[k]
\end{aligned}
$$

operando análogamente sobre $i_{b f}$ e $i_{c f}$, resulta la expresión compacta simultánea:

$$
\left[\begin{array}{l}
i_{a f} \\
i_{b f} \\
i_{c f}
\end{array}\right]_{K+1}=\frac{T_{S}}{3 L}\left(\left[\begin{array}{cc}
2 & 1 \\
-1 & 1 \\
-1 & -2
\end{array}\right]\left(\left[\begin{array}{l}
V_{a b} \\
V_{b c}
\end{array}\right]_{K}-\left[\begin{array}{l}
e_{a b} \\
e_{b c}
\end{array}\right]_{K}\right)\right)+\left(1-\frac{R T_{S}}{L}\right)\left[\begin{array}{l}
i_{a f} \\
i_{b f} \\
i_{c f}
\end{array}\right]_{K}
$$

El término de la izquierda son las corrientes calculadas hacia adelante en función de las variables muestreadas en el instante actual $k$. Esta ecuación se computa para los estados definidos por (3.7) y cada conjunto de corrientes calculadas debido a cada estado posible del convertidor se evalúa por medio de la función de costo (3.11). La misma expresa el error cuadrático medio global entre las corrientes calculadas y sus referencias normalizado al valor de corriente eficaz nominal del convertidor. Dicha normalización es necesaria debido a que la expresión de costo total (3.8) evalúa magnitudes de naturaleza diferente. Luego, cada uno de los estados definidos en (3.7) es evaluado y aquel que minimiza el valor de (3.11) resulta el más adecuado para seguir a la referencia de corriente:

$$
g_{I}[k+1]=\frac{1}{3} \sum_{j=a, b, c} \frac{\left(i_{j r e f}[k+1]-i_{j f}[k+1]\right)^{2}}{i_{R M S}^{2}}
$$

\subsubsection{Función de costo de balance}

Como se observa en (3.6), las mismas tensiones de línea producen idénticos cambios en las corrientes de salida. Sin embargo, un conjunto dado de tensiones de línea puede sintetizarse mediante uno o más conjuntos de tensiones de columna, repercutiendo cada 
uno de ellos de manera diferente sobre las tensiones de los capacitores del bus $D C$. En este caso, en forma similar a la adoptada en el Capítulo 2 para la modulación vectorial, se calculan los conjuntos de desviaciones de tensión sobre todos los capacitores del bus para cada una de las combinaciones admitidas por la expresión (3.7). Repitiendo el razonamiento y considerando en este caso la ausencia de una fuente de tensión continua en el bus $(F=0)$, tenemos:

$$
\left\{\begin{array}{l}
\Delta V_{C_{j}}=\left\{\begin{array}{cc}
\frac{T_{S} I_{m}\left(k T_{S}\right)}{C} & 1 \leq j \leq m-1 \\
0 & m \leq j \leq N-1
\end{array}\right. \\
V_{C_{j}}[k+1]=V_{C_{j}}[k]+\Delta V_{C j}[k+1]
\end{array}\right.
$$

La función de costo que mide el desequilibrio global del bus es:

$$
g_{V}=\frac{1}{N-1} \sum_{j=1}^{N-1} \frac{\left(V_{C r e f}-V_{C j}[k+1]\right)^{2}}{V_{C r e f}^{2}}
$$

En este caso, $\mathrm{gV}$ se normaliza al valor nominal de la tensión en cada capacitor en forma similar a la función de costo de corriente (3.11).

\subsubsection{Función de costo de reducción de frecuencia de conmutación}

Como variable secundaria a optimizar se incorpora la frecuencia de conmutación. Si bien la optimización de este parámetro no es fundamental para el funcionamiento del sistema, como sí lo son las corrientes y el balance, pequeñas desviaciones de las secuencias de conmutación pueden reducir las pérdidas por conmutación en los dispositivos y las emisiones electromagnéticas.

Una transición de conmutación puede llevarse a cabo cambiando el estado de una, dos o tres columnas, o bien ninguna (estado posterior igual al anterior). Entonces, la frecuencia media de conmutación de los dispositivos puede reducirse penalizando las transiciones de múltiples columnas en forma simultánea. Si se define $\mathrm{n}$ como el número de columnas que conmutan de un estado al otro, la función de costo asociada puede escribirse como en (3.14): 


$$
\cdot g_{n}[k+1]=\frac{1}{3} n
$$

Dicha función penaliza aquellas transiciones donde existe mayor número de columnas que conmuten a la vez.

\subsubsection{Diagrama de flujo del algoritmo de control}

La Fig. 3.4 muestra el diagrama del controlador FCS-MPC para el convertidor. En el instante $k T_{S}$ se aplica el estado de conmutación $\mathbf{V}[\mathrm{k}]$. En ese instante se muestrean las corrientes y las tensiones del bus $D C$ para el precálculo del próximo estado $\mathbf{V}[\mathrm{k}+1]$ a aplicar en el instante $(k+1) T_{S}$. La búsqueda comienza con la aplicación de (3.7) que define el subconjunto $U$ de estados de transición posibles. Este subconjunto poseerá como número máximo 27 elementos. Una vez determinado $U$ se procede al cálculo de las corrientes de línea y las tensiones de los capacitores del bus $D C$ (Ecs. (3.10) y (3.12)). Luego se computan las funciones de costo individuales (3.11), (3.13) y (3.14). A través del "pesado" de cada función de costo individual mediante los factores $K_{I}, K_{V}$, $K_{n}$ se integran todos en el cálculo de (3.8) y aquel que minimiza el resultado es elegido como el próximo estado del convertidor.

\subsection{Aplicación al filtrado activo de corrientes armónicas}

Como se describió en el capítulo 2, el convertidor con frente pasivo es incapaz de operar con alto factor de potencia y alto índice de modulación. Sin embargo, incluso en aplicaciones de procesamiento de potencia reactiva o de armónicas se requiere que el convertidor sea capaz de procesar potencia activa para compensar las pérdidas. Asimismo, es indispensable poder actuar sobre el balance de los capacitores en rechazo a perturbaciones o transitorios y por lo tanto, aún en estos casos, es necesario la utilización de un lazo de control que ejerza acción de balance sobre el bus. Bajo estas consideraciones se propuso utilizar un convertidor $D C M C$ de 5 niveles como filtro activo de potencia haciendo uso de la técnica FCS-MPC para el control integrado de las corrientes y las tensiones en los capacitores. Este esquema arriba a un esquema de control compacto y directo de todas las variables de interés que se integra en un único algoritmo y que no precisa moduladores externos. 


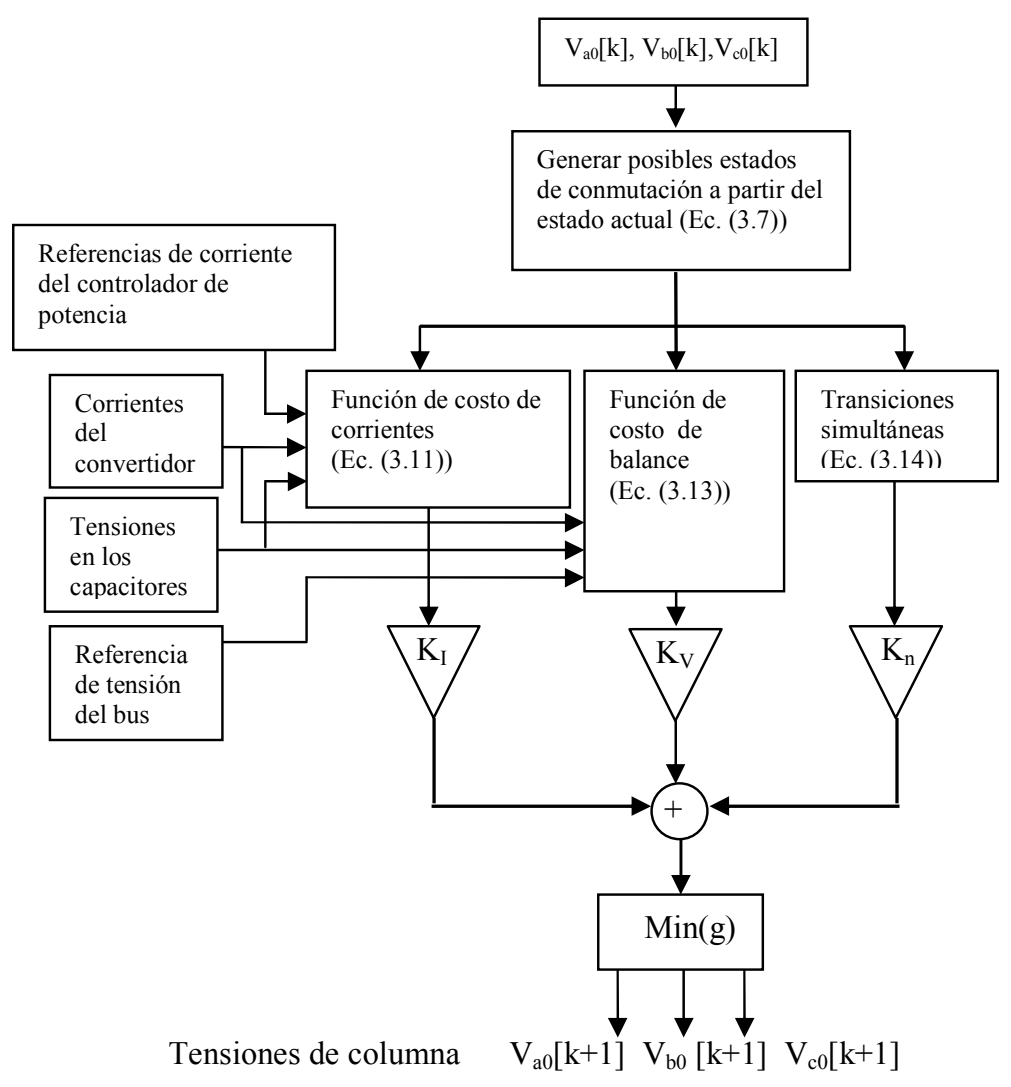

Figura 3.4. Diagrama de flujo del algoritmo.

La Fig. 3.5 muestra el diagrama de un sistema de compensación de corrientes armónicas y componente reactiva sobre una carga no lineal mediante un filtro activo paralelo $(F A P)$. El consumo de corrientes armónicas y/o reactivas circulando a través de la impedancia de línea $L_{S}$ origina una tensión de servicio distorsionada o disminuida sobre el nodo de conexión común $P C C$. Dicha distorsión implica una disminución en la calidad de la forma de onda que puede afectar a los demás consumidores, a la vez que la circulación de corrientes armónicas por los transformadores de la red genera calentamiento adicional de sus núcleos y la consecuente disminución en su capacidad operativa. Frecuentemente, en presencia de consumidores medianos las perturbaciones se introducen en forma directa en la red de media tensión. En tal caso, el sistema de compensación debe ser capaz de trabajar en ese rango de tensiones con acoplamiento directo o bien hacerlo a niveles de baja tensión mediante transformador elevador. La posibilidad de omitir el transformador de acoplamiento trae como ventajas la reducción de las pérdidas, el peso y volumen total del convertidor a la vez que se elimina un 
componente de alto costo. En este sentido el convertidor $D C M C$ es una alternativa interesante como interfaz de potencia para dicha aplicación.

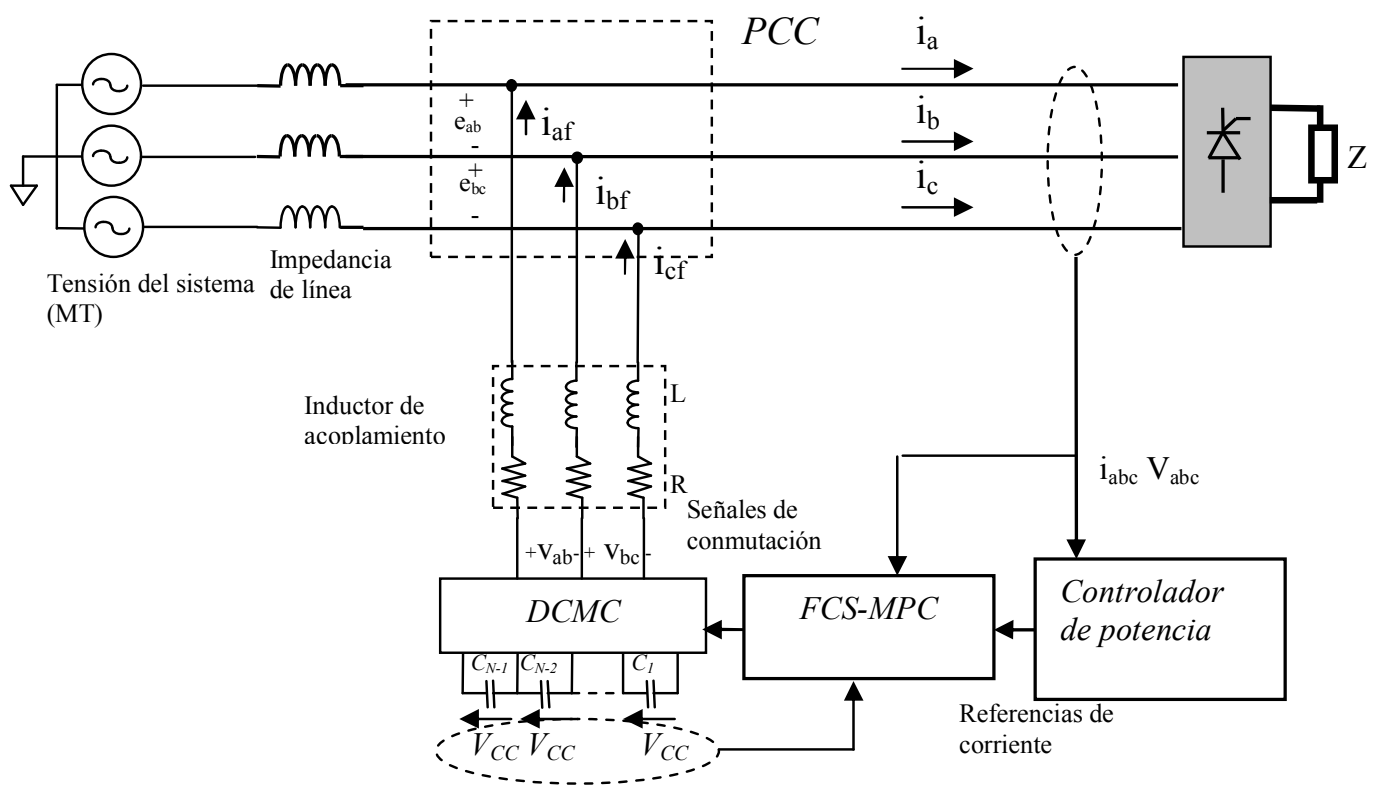

Figura 3.5. Topología de conexión del FAP.

La carga no lineal consume una corriente compuesta por corriente activa, reactiva y también armónicas. El objetivo del $F A P$ consiste en proveer la reactiva y las armónicas tal que la red sólo entregue corriente sinusoidal en fase con la tensión.

El controlador de potencia posee una estructura feed-forward y se basa en la teoría de la potencia instantánea [123]. Según esta teoría, las potencias activa y reactiva pueden descomponerse en sus partes fundamentales y armónicas como se describe en (3.15), cuyas variables son las corrientes y tensiones transformadas al plano $\alpha \beta$ mediante (2.1) desestimando la componente homopolar:

$$
\left[\begin{array}{l}
p \\
q
\end{array}\right]=\left[\begin{array}{c}
\bar{p}+\tilde{p} \\
\bar{q}+\tilde{q}
\end{array}\right]=\left[\begin{array}{cc}
v_{\alpha} & v_{\beta} \\
-v_{\beta} & v_{\alpha}
\end{array}\right]\left[\begin{array}{c}
i_{\alpha} \\
i_{\beta}
\end{array}\right]
$$

La Ec. (3.15) expresa la potencia instantánea real $p$ e imaginaria $q$ drenadas por la carga contaminante. El carácter no lineal de la misma origina componentes no nulas de potencia activa armónica $\tilde{p}$, potencia reactiva armónica $\tilde{q}$ y potencia reactiva de frecuencia fundamental $\bar{q}$ las cuales deben ser suministradas por el filtro activo en el punto de conexión común. El controlador de potencia calcula las referencias de 
corriente del FAP para el FCS-MPC a fin de realizar la compensación. La Fig. 3.6 muestra el flujo de cálculo interno de controlador.

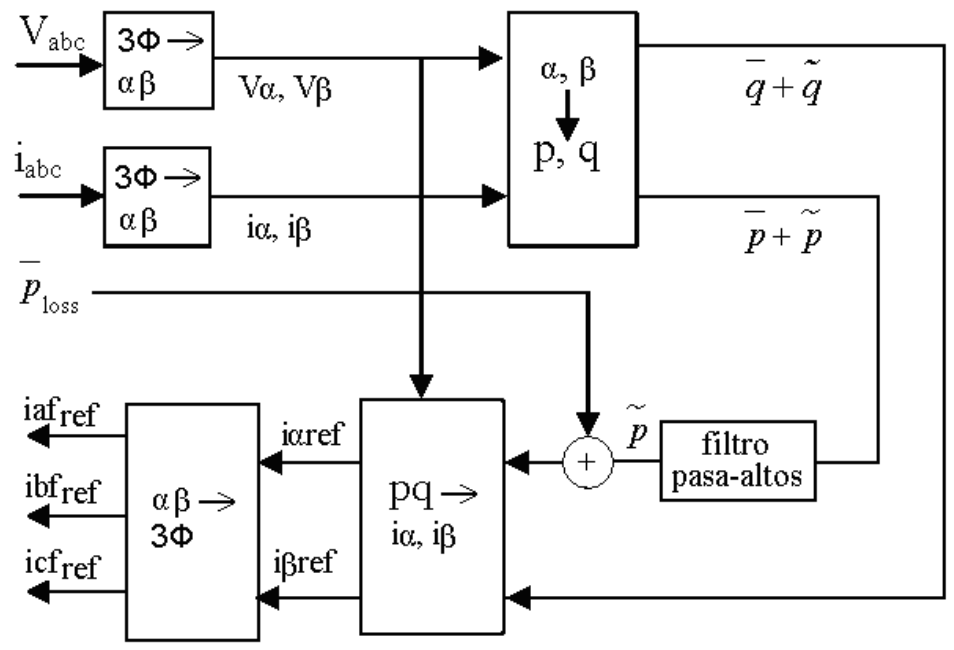

Figura 3.6. Controlador de Potencia

Las corrientes de referencia expresadas en el plano $\alpha \beta$ se calculan mediante la relación inversa:

$$
\left[\begin{array}{l}
i_{\text {oref }} \\
i_{\beta r e f}
\end{array}\right]=\frac{1}{v_{\alpha}^{2}+v_{\beta}^{2}}\left[\begin{array}{cc}
v_{\alpha} & -v_{\beta} \\
v_{\beta} & v_{\alpha}
\end{array}\right]\left[\begin{array}{l}
p \\
q
\end{array}\right]
$$

Éstas son a su vez transformadas a las corrientes de línea $i_{\text {afref }}, i_{b f r e f}$ e $\mathrm{i}_{\text {cfref }}$ mediante la transformación inversa $T_{\alpha \beta 0}^{-1}$ e ingresadas a la función de costo (3.11) para la selección del próximo estado del convertidor.

La carga contaminante consistió en un rectificador controlado de tiristores que introduce a la red una corriente altamente distorsionada. La estabilización del bus de continua del $D C M C$ se logró a partir de un controlador $P I$ externo que introduce al controlador de potencia la componente activa a frecuencia fundamental para estabilizar la tensión del bus. Este valor se fijó en $20 \mathrm{kV}$. La frecuencia de muestreo se estableció en $10 \mathrm{kHz}$, mientras que los parámetros del inductor de acoplamiento son $R=5 \mathrm{~m} \Omega$ y $L=8 \mathrm{mH}$, y la capacidad es $C=4700 u F$. Los factores de peso para el algoritmo predictivo se ajustan a $K_{I}=1, K_{V}=0,1, K_{n}=0,001$ ya que los objetivos principales son el control de corriente y el 
balance de tensiones, y en menor medida la frecuencia de conmutación de los dispositivos.

La Fig. 3.7 muestra las tensiones de línea del sistema y del convertidor. Para el seguimiento óptimo de las corrientes el convertidor sintetizó formas de onda suaves con saltos entre niveles adyacentes. La Fig. 3.8 muestra las tensiones de los capacitores y las tensiones de columna referidas al negativo del bus de continua. Al comienzo, se impuso una condición inicial de desbalance en los mismos, observándose la eficacia del término $K_{V} g_{V}$ el cual restablece la tensión de referencia $(5 \mathrm{kV})$ en aproximadamente $200 \mathrm{~ms}$.
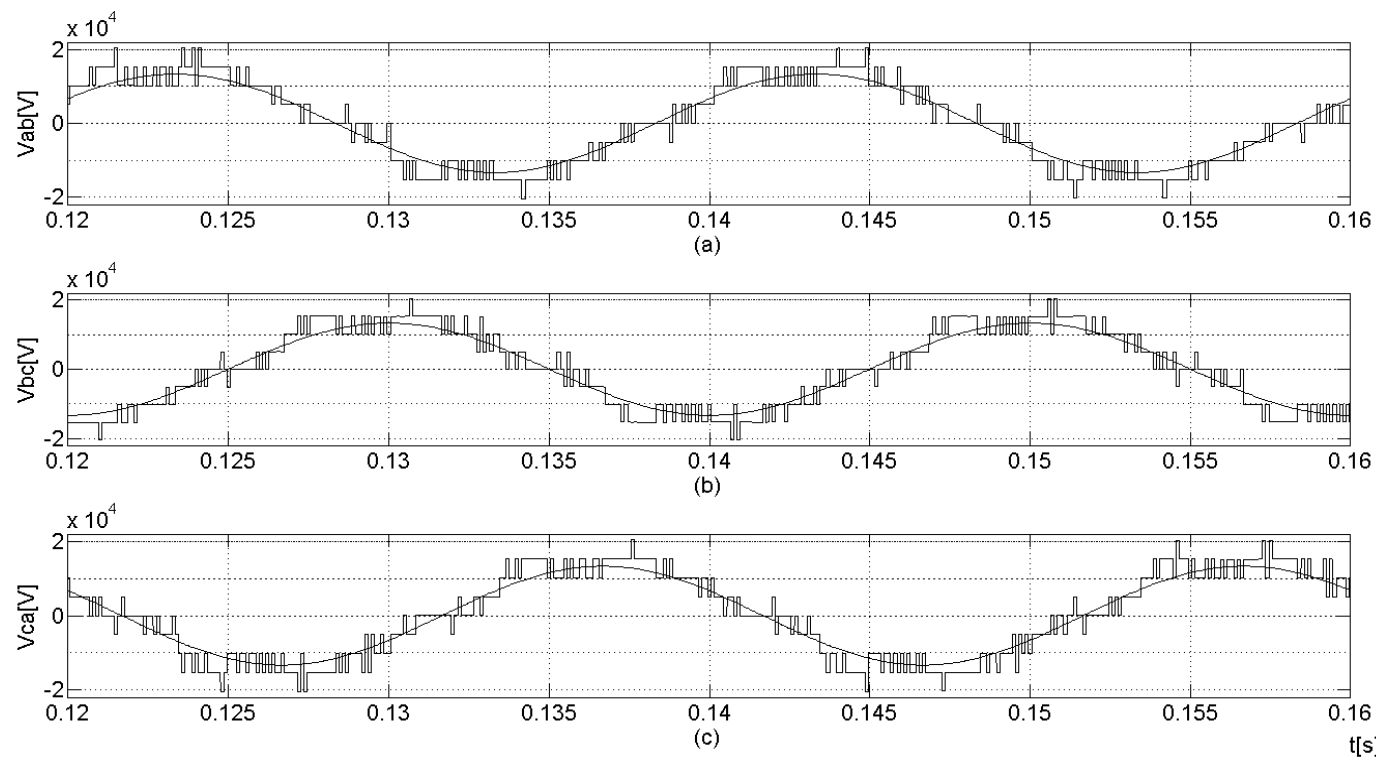

Figura 3.7. Tensiones de línea del sistema y a la salida del convertidor (a) $V_{a b}, e_{a b}$, (b) $V_{b c}, e_{b c},(c) V_{c a}, e_{c a}$.

La Fig. 3.9 muestra en detalle una región de los trazos anteriores en donde pueden verificarse las transiciones unitarias. Además se aprecia el valor del rizado en la tensión de los capacitores con un valor aproximado de $70 V_{p p}$, el cual representa un $1,5 \%$ del valor nominal de tensión de referencia. Por otra parte puede observarse que la frecuencia de conmutación no es fija para los dispositivos. Esta es una característica del algoritmo $F C S-M P C$ ya que al no existir una portadora los dispositivos no están obligados a conmutar a una frecuencia definida. Observando la Fig. 3.9(c) pueden contabilizarse la cantidad de conmutaciones de un nivel a otro que para el caso de los dispositivos internos se encuentra en el orden de $1 \mathrm{kHz}$. 
Las Figs. 3.10(a) y 3.10(c) muestran las corrientes de referencia $i_{a f}$ e $i_{c f}$ provenientes del controlador de potencia y las correspondientes variables medidas. El error absoluto de las corrientes se muestra en las figuras 3.10 (b) y $3.10(\mathrm{~d})$. Durante el trazo suave de las corrientes, dicho error se mantiene alrededor de $20 \mathrm{~A}(4 \%)$, mientras que en las transiciones bruscas debido a la rápida conmutación de corriente en la carga contaminante, éste aumenta a $50 \mathrm{~A}$, lo cual representa el $10 \%$ del valor pico de corriente.
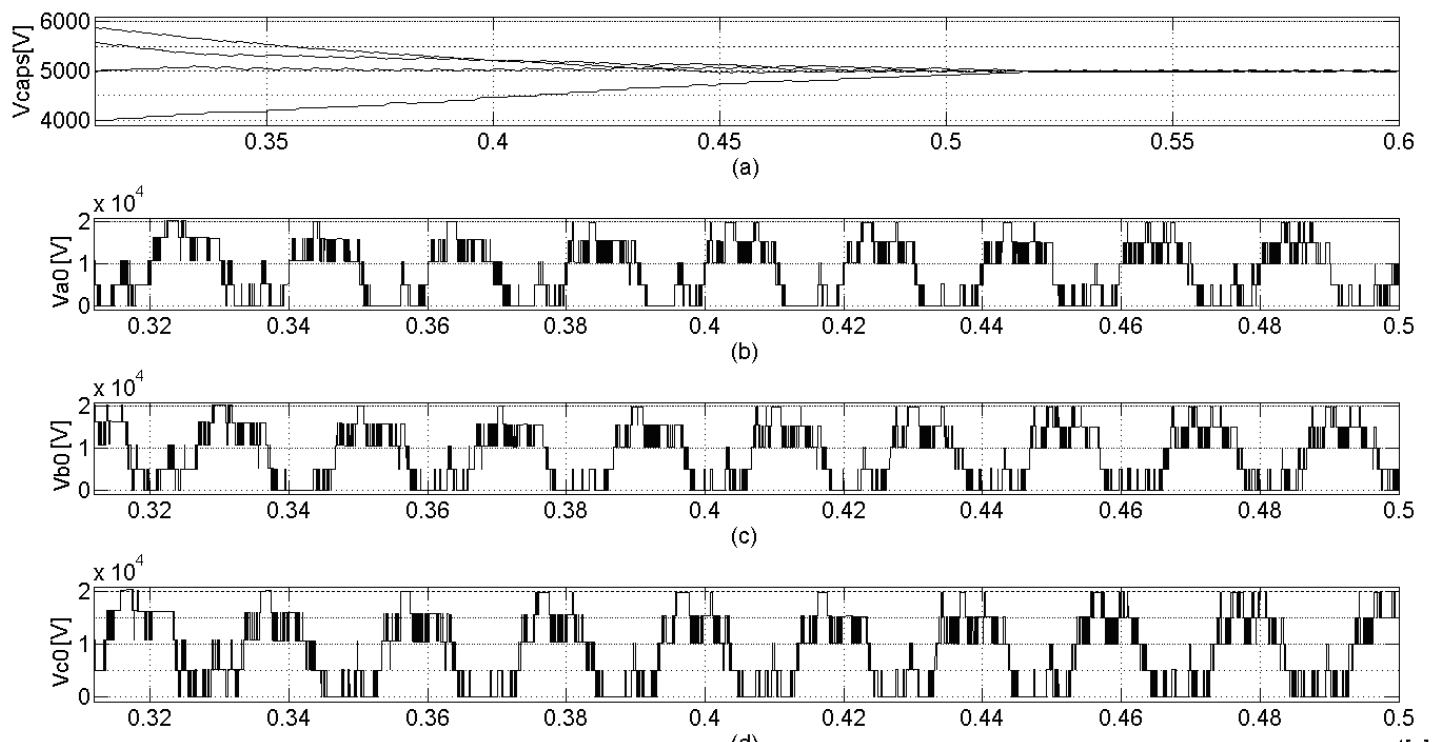

Figura 3.8. (a) Tensiones en los capacitores, (b) Tensión de columna $V_{a}$, (c) Tensión de columna $V_{b}$, (d) Tensión de columna $V_{c}$.
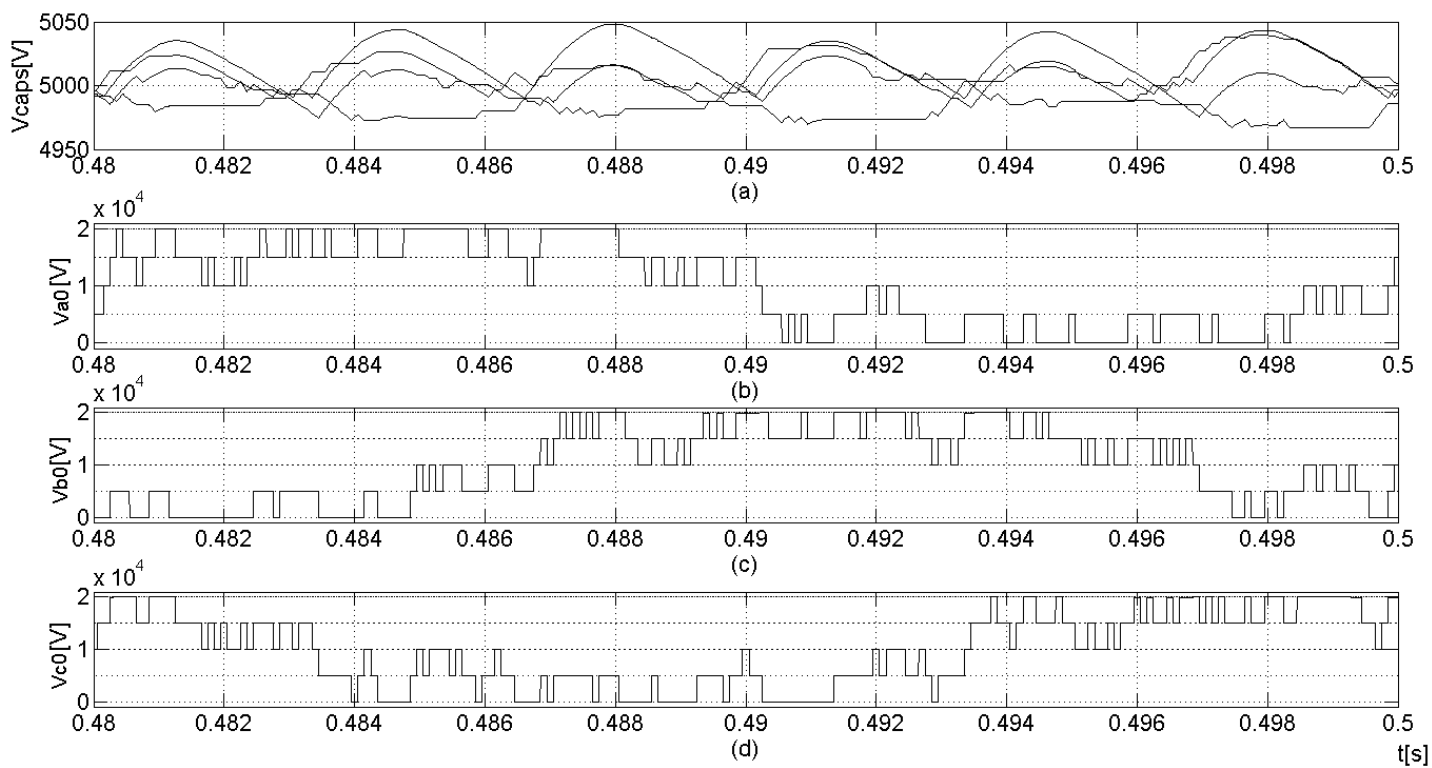

Figura 3.9. Detalle de formas de onda de tensión (a) Tensiones en los capacitores, (b) Tensión de columna $V_{a}$, (c) $V_{b} y(d) V_{c}$. 
La Fig. 3.11(a) muestra la corriente drenada por la carga y la inyectada por el convertidor. La componente reactiva y también las armónicas son detectadas e inyectadas para la cancelación en el nodo de compensación. La tensión de fase del sistema y su correspondiente corriente de línea se muestran en la Fig. 3.11(b). La corriente se presenta sinusoidal con factor de potencia unitario y baja distorsión. La distorsión residual de baja frecuencia es consecuencia de la relación de compromiso entre el valor del inductor de acoplamiento, el ripple de corriente y el valor de tensión del bus $D C$.

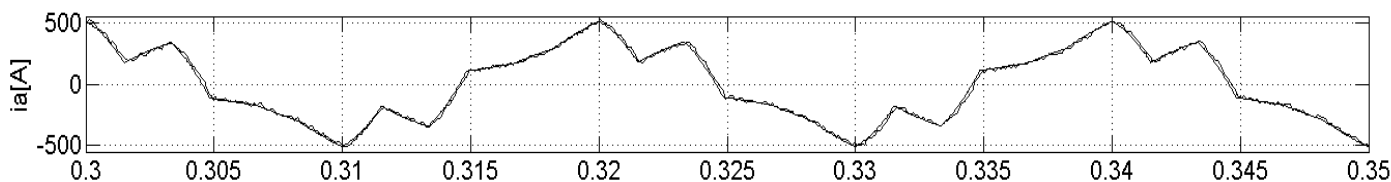

(a)
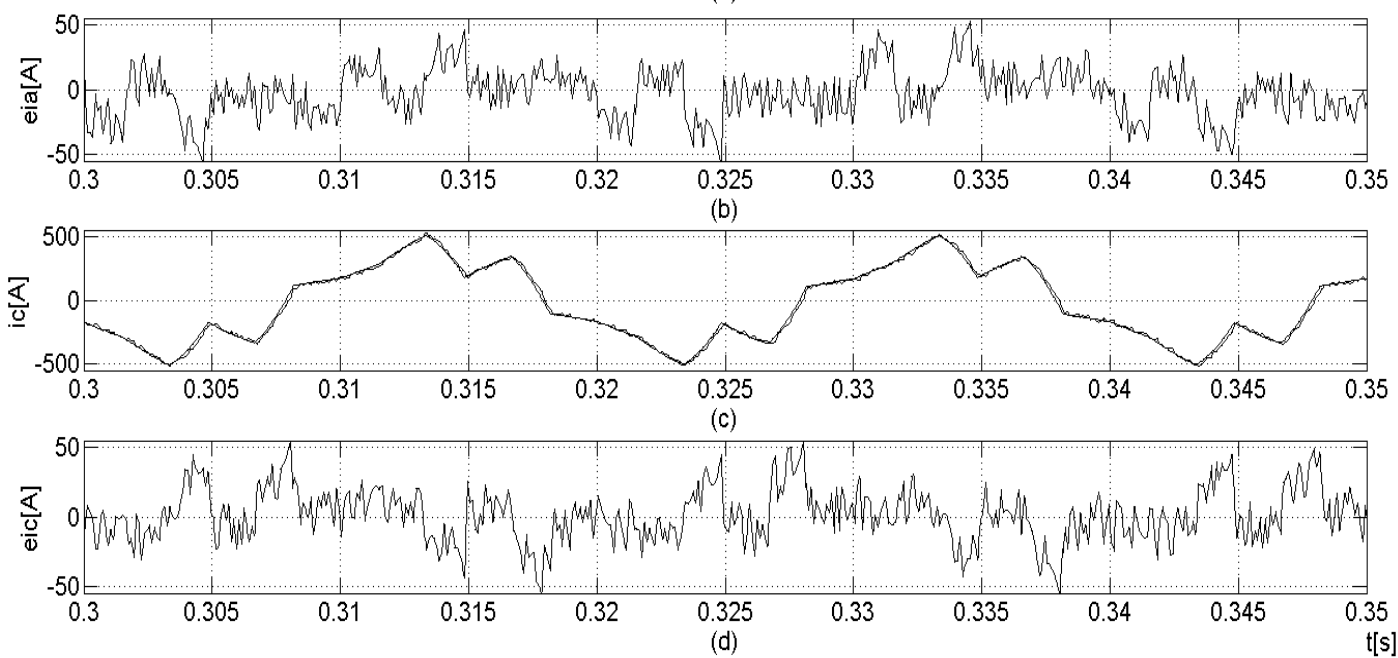

Figura 3.10. Corrientes de referencia y corrientes medidas a la salida del convertidor (a) $i_{\text {aref }} y i_{\text {af }}$ (superpuestas), (b) error $\left(i_{\text {aref }}-i_{a f}\right)$, (c) $i_{\text {cref }} y i_{c f}$ (superpuestas), (b) $\operatorname{error}\left(i_{c r e f}-i_{c f}\right)$. 

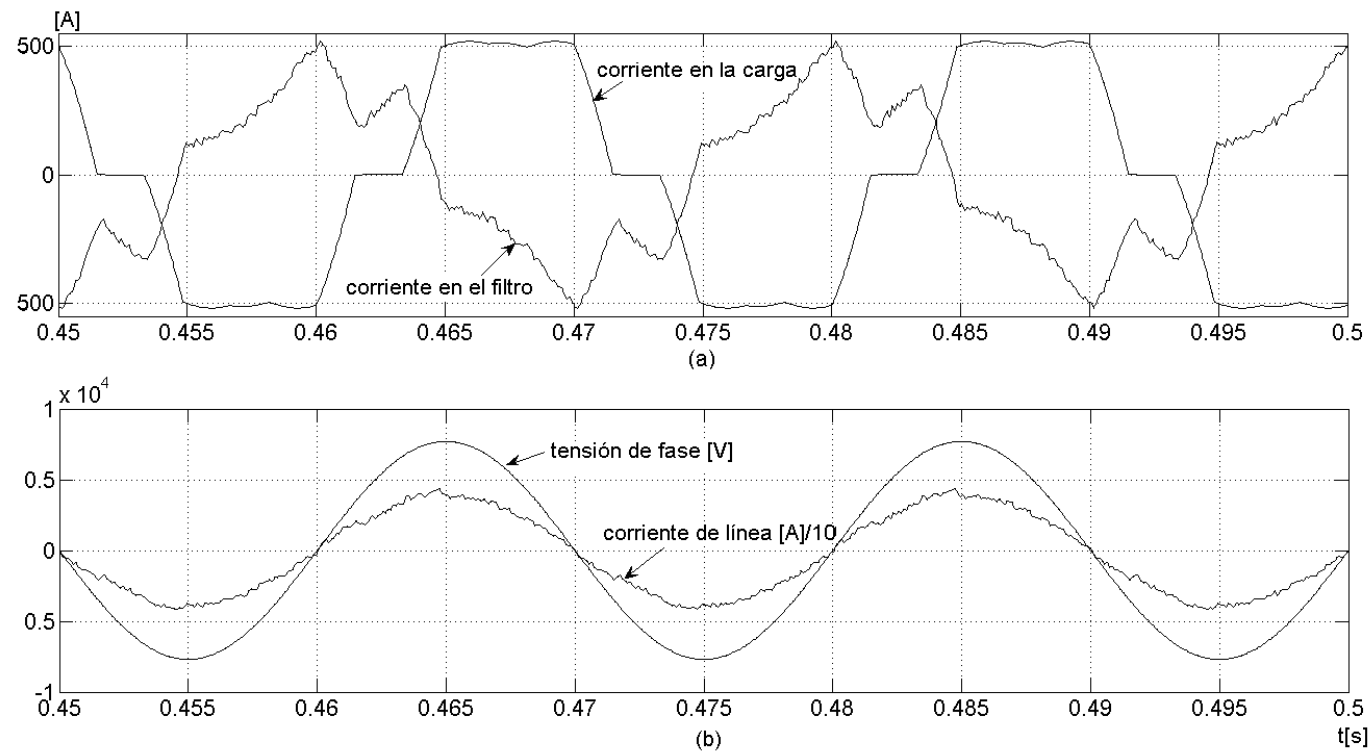

Figura 3.11. Una tensión de fase y corriente de línea del sistema (a) Corriente sobre la carga y a la salida del convertidor, (b) Tensión y corriente de fase del sistema.

Finalmente, en la Fig. 3.12 se muestra el comportamiento del sistema cuando una carga adicional es conectada en el lado de continua del convertidor de tiristores. En $t=0,32 \mathrm{~s}$ la impedancia de carga en el lado de continua del convertidor de tiristores de valor $Z$ fue reducida a $Z / 2$ (Fig. 3.5). La Fig. 3.12(a) muestra las tensiones de los capacitores, las cuales permanecen en condición de balance aún durante el transitorio. En este lapso el control PI restableció el valor de la tensión del bus. En las Figs. 3.12(b) y 3.12(c) la corriente suministrada por la red posee forma sinusoidal a la vez que se observa la inyección de las componentes armónicas necesarias por parte del FAP. En estas condiciones también se cumplió estrictamente la condición de transiciones unitarias en la tensión de columna, como se observa en las Figs. 3.13(b), (c) y (d). 


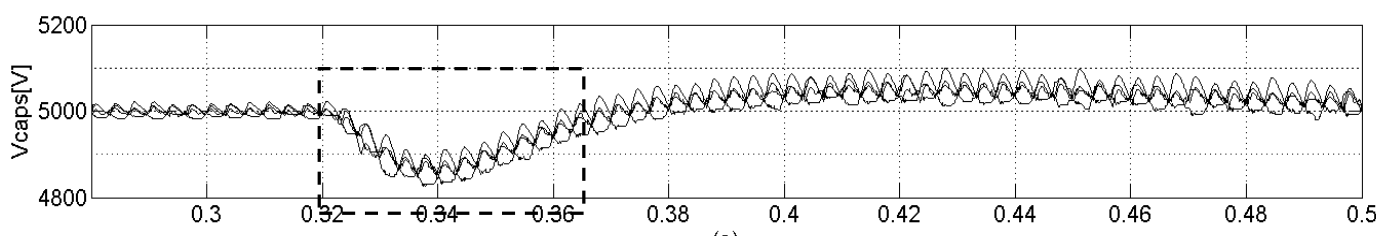

(a)

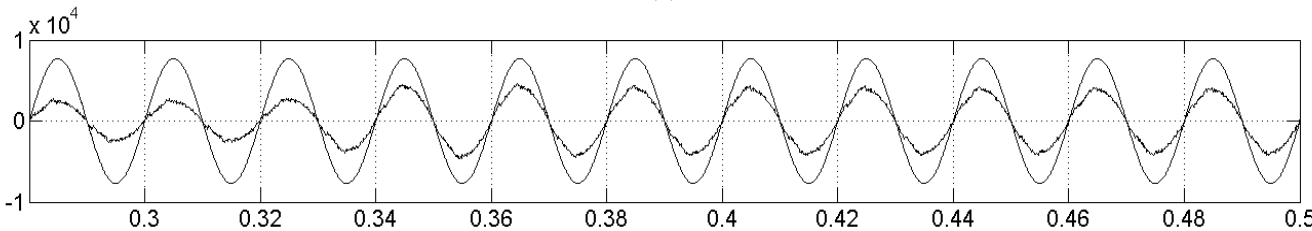

(b)

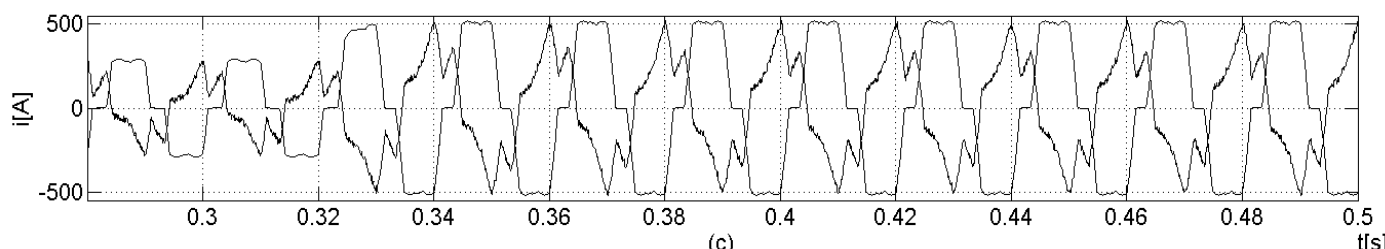

Figura 3.12. Formas de onda en respuesta a un cambio brusco de la carga alineal. (a) Tensiones en los capacitores, (b) Tension de fase y corriente de línea (c) Corriente en la carga y corriente de salida del convertidor.
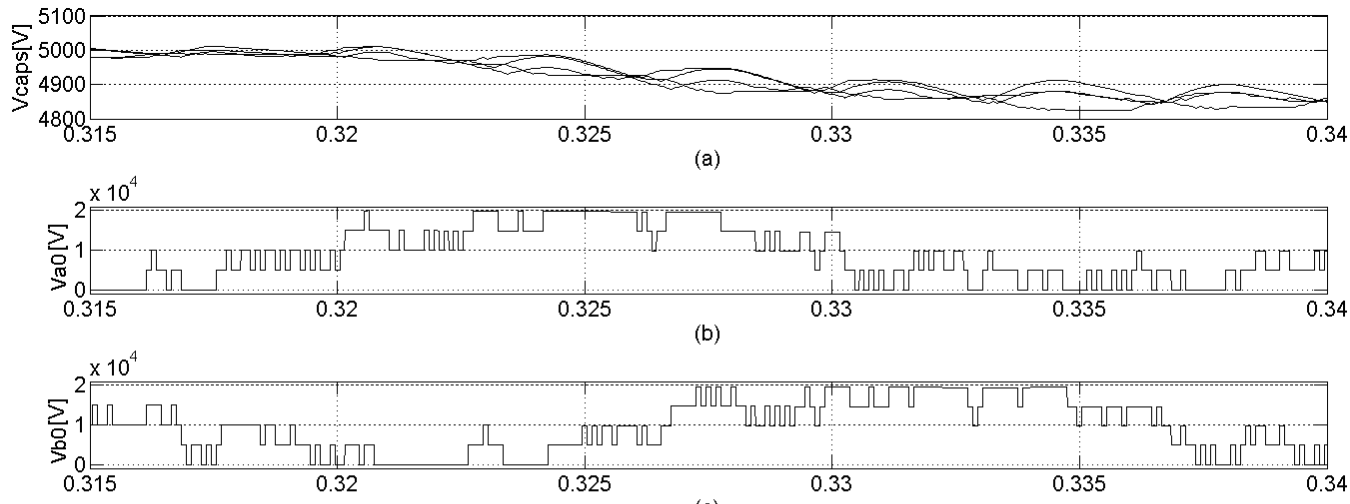

(c)

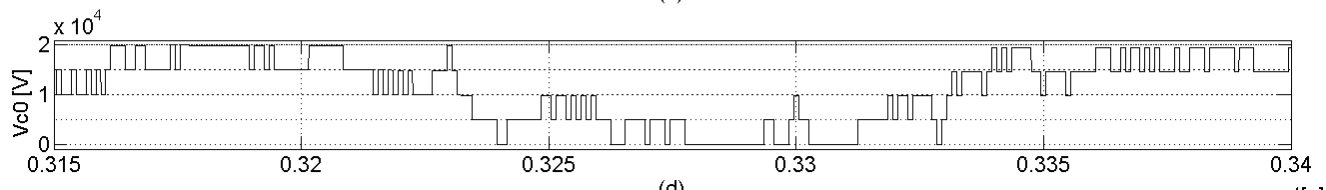

(d) $\mathrm{t}[\mathrm{s}]$

Figura 3.13. Detalle de formas de onda en respuesta a un cambio brusco de la carga alineal. (a) Tensiones en los capacitores, (b)(c)(d) Tensiones de columna.

\subsection{Conclusiones}

El presente capítulo introdujo la técnica de control predictivo para convertidores de potencia. Se desarrolló un algoritmo general para el control directo de las corrientes del convertidor multinivel con enclavamiento por diodos que permite la incorporación del 
control de balance de tensión sobre los capacitores del bus de continua. Esto asegura el equilibrio de los niveles de tensión en el bus permitiendo bajo nivel de distorsión en la tensión de salida. En base a las consideraciones aportadas en el capítulo anterior se sintetizó una regla explícita para la conmutación de las columnas del convertidor, la cual proporciona transiciones seguras entre los estados de conmutación. El esquema desarrollado incorpora internamente dichas restricciones reduciendo el número de estados a evaluar llevando este número a un valor constante de 27 estados (igual al de un convertidor de tres niveles), independientemente del número de niveles del convertidor considerado. El algoritmo es naturalmente digital y no requiere moduladores dentro del lazo de control. Se aplicó la estrategia propuesta en un filtro activo de corriente reactiva y armónicas de una carga contaminante. 


\section{Capítulo 4}

\section{$\underline{\text { El convertidor DCMC con frente activo }}$}

\subsection{Introducción}

Más del 50\% del consumo de energía eléctrica es destinada a accionamientos eléctricos y la utilización de variadores de velocidad para motores de $C A$ aumenta en forma constante. Esto se observa en instalaciones industriales e incluso en artefactos de uso cotidiano como lavarropas y equipos de aire acondicionado [124]. El uso de variadores de velocidad ofrece gran flexibilidad para el control de procesos como así también excelentes resultados en términos de ahorro de energía. También permite la extensión de la vida útil de componentes gracias a la posibilidad de eliminar lazos de regulación mecánicos, como por ejemplo aquellos basados en válvulas de obturación para regulación de caudal. Además, el estudio permanente de las máquinas de $C A$ y el desarrollo de algoritmos de control sofisticados extienden su uso a aplicaciones de alto desempeño para todas las escalas de potencia [125]. En este sentido, gracias a su robustez y confiabilidad, el motor de inducción (y actualmente también el motor sincrónico de imanes permanentes) en conjunto con los convertidores de potencia invaden campos de aplicación de dominio histórico exclusivo de los motores de corriente continua.

La conversión de frecuencia mediante convertidores indirectos con fuente de tensión requiere la rectificación de las tensiones de la red. Esto se realiza, en general para potencias menores a $30 \mathrm{~kW}$ mediante rectificación a diodos, reemplazándose estos por puentes de tiristores para potencias mayores. Ambas configuraciones presentan limitaciones como ser: 1) la tensión máxima resultante en el bus $D C$ no es suficiente para alcanzar condiciones nominales de salida en el inversor sin sobremodular y 2) sólo 
permiten flujo unidireccional de potencia impidiendo la acción de frenado regenerativo. Este esquema también causa distorsión de la tensión de red debido a la inyección de componentes armónicas de corriente de baja frecuencia, degradando la confiabilidad de equipos sensibles conectados en el punto de conexión común [126]. En este sentido, si bien a potencias moderadas y altas, los estándares de contaminación armónica (IEEE519) pueden ser cumplidos mediante el uso rectificadores multipulso (Fig.4.1), este tipo de soluciones son voluminosas y pesadas debido a que requieren transformadores operando a frecuencia de red. Por otra parte, por encima de 12 pulsos, el transformador posee una configuración especial de devanados en zig-zag de complicada fabricación y aún así, se trata de dispositivos unidireccionales que no permiten intercambio de potencia entre ambos terminales $A C$.
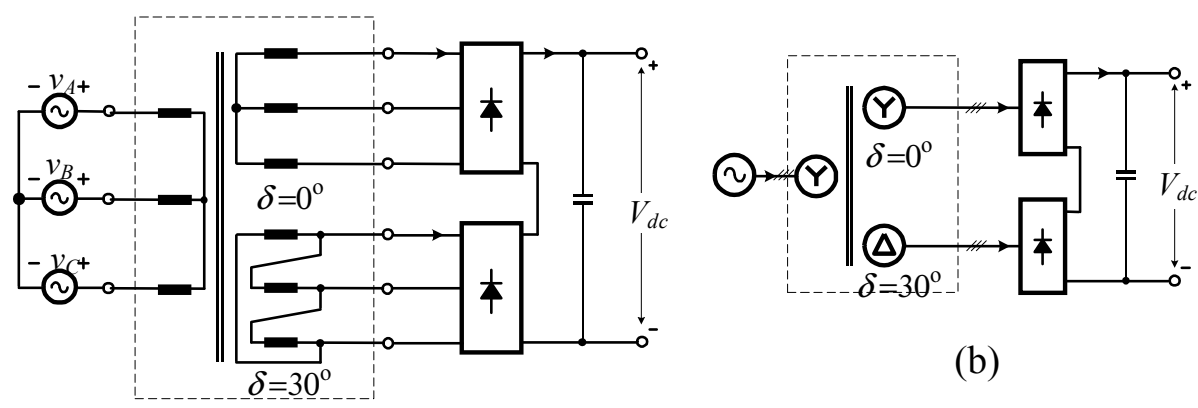

(b)

(a)

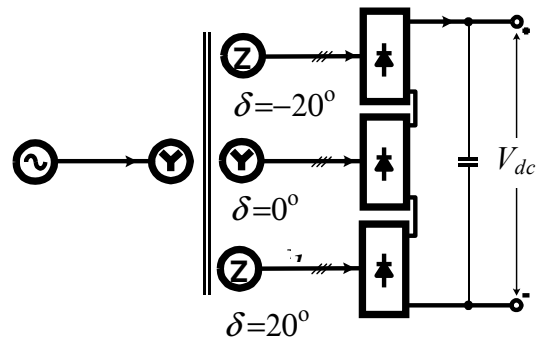

(c)

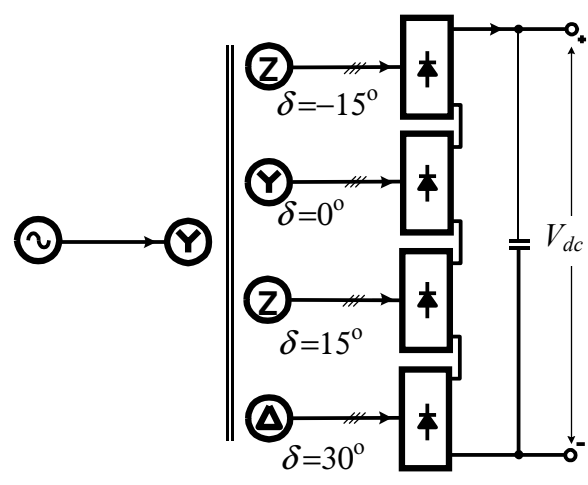

(d)

Figura 4.1. Rectificadores multipulso. (a)Rectificador de 12 pulsos, (b) Diagrama simplificado del rectificador de 12 pulsos, (c) Rectificador de 18 pulsos, (d) Rectificador de 24 pulsos.

La conexión back to back de convertidores alimentados con tensión (VSC) consiste en dos convertidores conectados a un bus de tensión continua común (Fig. 4.2). Debido al completo desacoplamiento entre ambos terminales de alterna, esta topología presenta gran versatilidad en cuanto a la independencia de control de las variables de la entrada y de la carga, y posee capacidad de intercambio bidireccional del flujo de potencia con 
bajo contenido armónico de corriente. Por otra parte, el convertidor de entrada $(C E)$ permite elevar la tensión del bus de continua a un valor superior a la tensión de línea del sistema pudiendo, en el caso de accionamiento de un motor, alcanzar el valor de potencia nominal de la máquina [127]. Por último, en conjunto con un lazo de control de tensión de bus suficientemente rápido, esta topología permite la reducción del capacitor de almacenamiento con respecto al requerido por un puente de diodos o de tiristores [47].

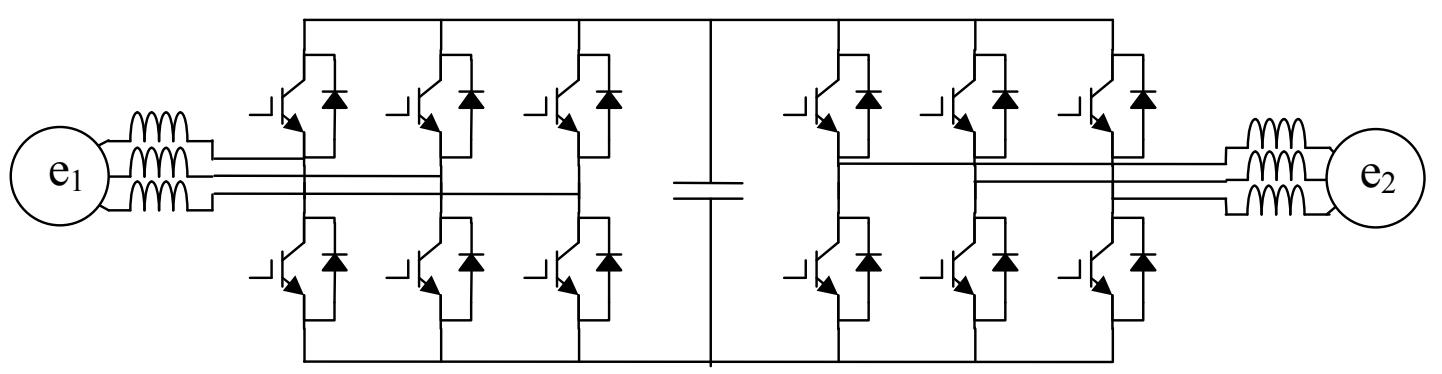

Figura 4.2. Topología back to back con convertidores de dos niveles

Estas características hacen a esta topología adecuada para una multiplicidad de aplicaciones como re-inyección de potencia en sistemas de frenado regenerativo [128], dispositivos de almacenamiento rotante [129], accionamiento de grandes compresores, bombas y laminadoras en la industria del petróleo y del acero, generación eólica [17] y también en transporte férreo y naval. También encuentra aplicaciones en FACTS y $C P$ como el UPQC, en transmisión DC (HVDC LIGHT) y generación distribuida [15].

La conexión directa de los convertidores a la red de media tensión tiene la ventaja de omitir el transformador de acoplamiento, y en este sentido, las topologías multinivel son especialmente atractivas. En particular en lo que se refiere a accionamientos, la síntesis de tensiones con múltiples niveles reduce los valores de $d V / d t$ sobre los devanados del motor, lo que reduce a su vez los efectos de sobretensiones en bornes debido a reflexiones. Esto repercute favorablemente sobre dos aspectos clave en la utilización de variadores de velocidad que son las fallas de aislación de los bobinados y en rodamientos debido a corrientes capacitivas parásitas e inducción de campo magnético longitudinal sobre el rotor de las máquinas [130]. En este sentido, el convertidor multinivel con enclavamiento por diodos es una topología apropiada para la conexión back to back [50]. Por otra parte, como se ha visto en el Capítulo 2, el convertidor $D C M C$ con frente pasivo posee una región limitada de funcionamiento cuyos límites 
están determinados por la relación entre el índice de modulación y el factor de potencia de la carga, independientemente del algoritmo de modulación utilizado. En este sentido, varios trabajos demuestran que la conexión back to back de convertidores DCMC proporciona una alternativa para operar con alto índice de modulación y factor de potencia sin aumentar el número de componentes del sistema $[131][132][133][134][135]$.

En el presente capítulo se realiza el análisis de una estrategia de control para el convertidor multinivel con enclavamiento por diodos en configuración back to back. Se analiza el efecto complementario que ejercen ambos convertidores sobre las tensiones del bus de continua y se propone un esquema de control autónomo para ambos utilizando la técnica $F C S-M P C$. En particular, se explora el convertidor en una aplicación de accionamiento de un motor de inducción de $M T$ mediante control predictivo directo de par. Los principales aportes del capítulo se encuentran publicados en los trabajos [67][68][69] y [70].

\subsection{Conexión $B 2 B$ sobre el $D C M C$. Principio de balance con bus compartido}

La Fig. 4.3 muestra el esquema de un sistema de conversión $C A / C C / C A$ que consiste en dos convertidores $D C M C$ en conexión back to back. Ambos terminales de alterna se modelan en forma general como sistemas trifásicos con acoplamiento inductivo. Dicha representación permite describir indistintamente un sistema trifásico con inductor de acoplamiento, o el estator de un motor con sus inductancias de dispersión y la fuerza contra electromotriz de sus bobinados.

Para cualquiera de las aplicaciones mencionadas, las características operativas que debe exhibir el sistema se describen a continuación:

- Operación en condiciones arbitrarias de índice de modulación y factor de potencia en ambos convertidores, sin restricciones de dependencia mutua.

- Capacidad de control de tensiones o corrientes en el convertidor de carga.

- Estabilidad del balance del bus de continua en las condiciones de funcionamiento previstas. 
- Capacidad de control de la potencia reactiva sobre el convertidor conectado a la red.

- Bajo nivel de distorsión en la corriente de ambos convertidores.

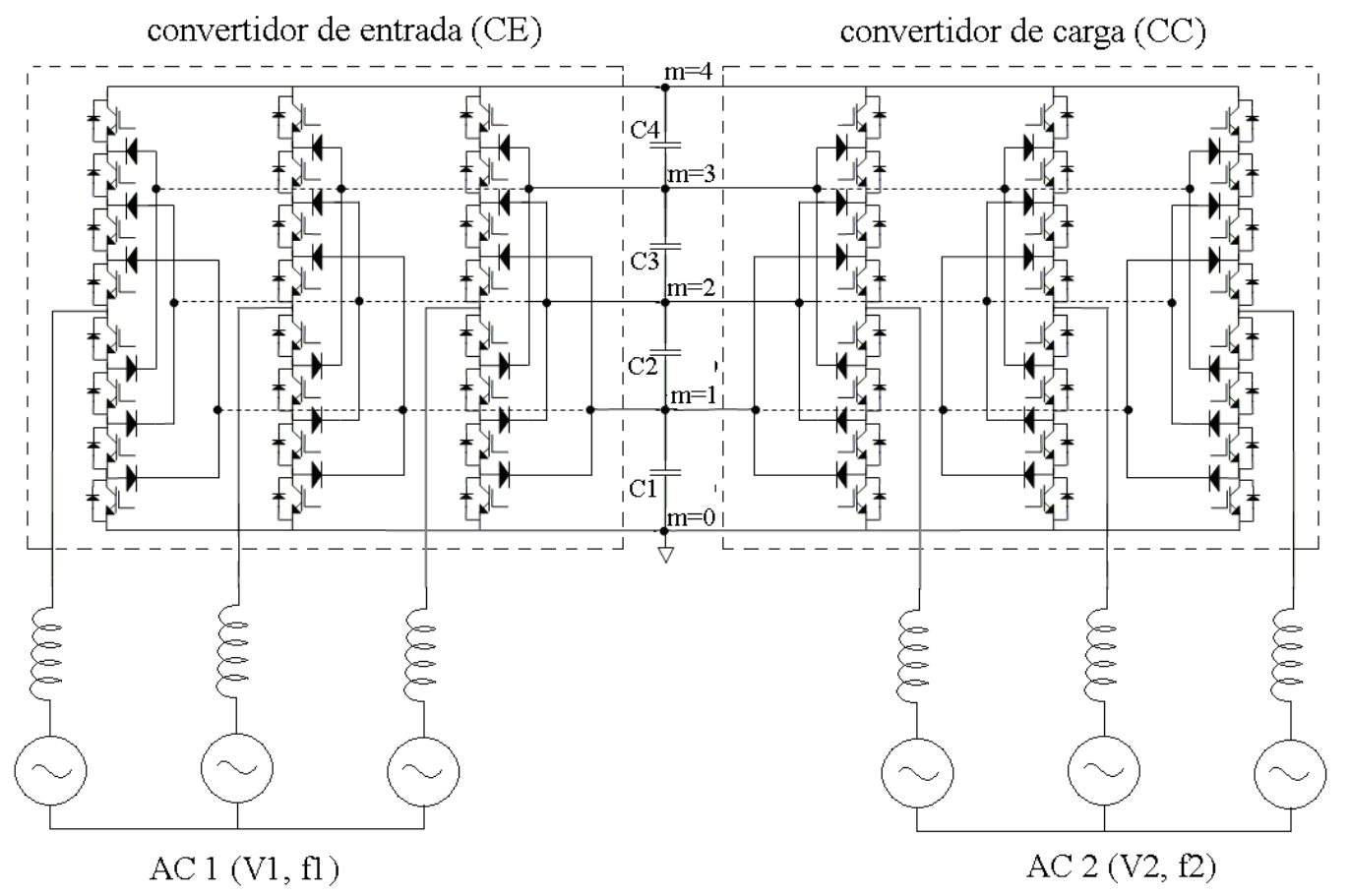

Figura 4.3. Convertidor back to back de 5 niveles basado en convertidores DCMC.

Según lo visto en el Capítulo 2, cuando el convertidor DCMC con frente pasivo opera como inversor, es decir que la potencia fluye desde el lado de continua al lado de alterna, los capacitores internos se descargan y los externos se cargan (Fig. 2.23(a)). Por el contrario, si opera como rectificador, los capacitores internos se cargan y los externos se descargan (Fig. 2.23(b)). Si el valor de potencia transferida es similar en ambos casos, la tasa de carga y descarga es la misma. En consecuencia la conexión back to back es una opción para extender la región de estabilidad del balance del bus de continua sin utilizar elementos adicionales [136]. Es decir que mientras el convertidor de entrada $(C E)$ tiende a cargar los capacitores internos del bus y descargar los externos, el convertidor de carga $(C C)$ tiende a descargar los capacitores internos y cargar los externos. Esto puede entenderse en términos de un carácter complementario entre las combinaciones de conmutación de ambos convertidores. Sin embargo, es importante destacar que la conexión back to back no asegura por sí misma el balance y por lo tanto es necesario el diseño cuidadoso de una estrategia de selección de los estados de 
conmutación para alcanzar este objetivo [131]. En este sentido, si las estrategias de control de ambos convertidores permiten explotar sus estados redundantes, el efecto opuesto de carga/descarga de ambos convertidores permite una contribución sinérgica en el balance de tensión de los capacitores del bus de continua. Se observa amplia evidencia experimental en este sentido [132][133][134] con varios esquemas de modulación: modulación sinusoidal con control de offset de portadoras [137], modulación programada con balance de tensión [132], modulación vectorial coordinada entre ambos convertidores [134] y comparadores dinámicos con histéresis [135][138].

\subsection{Control de los convertidores}

El objetivo del $C E$ (Fig. 4.3) consiste en la estabilización de la tensión del bus de continua frente a las variaciones de flujo de potencia y a las perturbaciones de la red eléctrica. Por su parte, el $C C$ entrega potencia a través del control de las corrientes o tensiones. A su vez, ambos convertidores deben ejecutar un lazo de control de balance del bus $D C$ a fin de preservar los dispositivos de conmutación ante tensiones de bloqueo excesivas. Se advierte entonces una multiplicidad de magnitudes a controlar lo cual sugiere la aplicación de esquemas de control multivariable.

La estrategia de control predictivo desarrollada en el Capítulo 3 se utiliza también para el control del convertidor DCMC en conexión back to back. En esta propuesta cada convertidor ejecuta su propio algoritmo en forma autónoma e independiente, omitiendo el intercambio de información entre ambos respecto de sus estados de conmutación. Esto permite descentralizar la ejecución de las rutinas de control o bien paralelizarlas disminuyendo el requerimiento computacional a nivel de implementación. Por otra parte, cada algoritmo predictivo posee funciones de costo diferentes orientadas hacia el control de las variables externas al convertidor y también al balance del bus $D C$.

La Fig. 4.4 muestra el diagrama en bloques de un convertidor back to back con convertidores DCMC utilizado como accionamiento de un motor de inducción. El conjunto se alimenta desde la red de suministro de media tensión en forma directa y sin transformador de acoplamiento. 


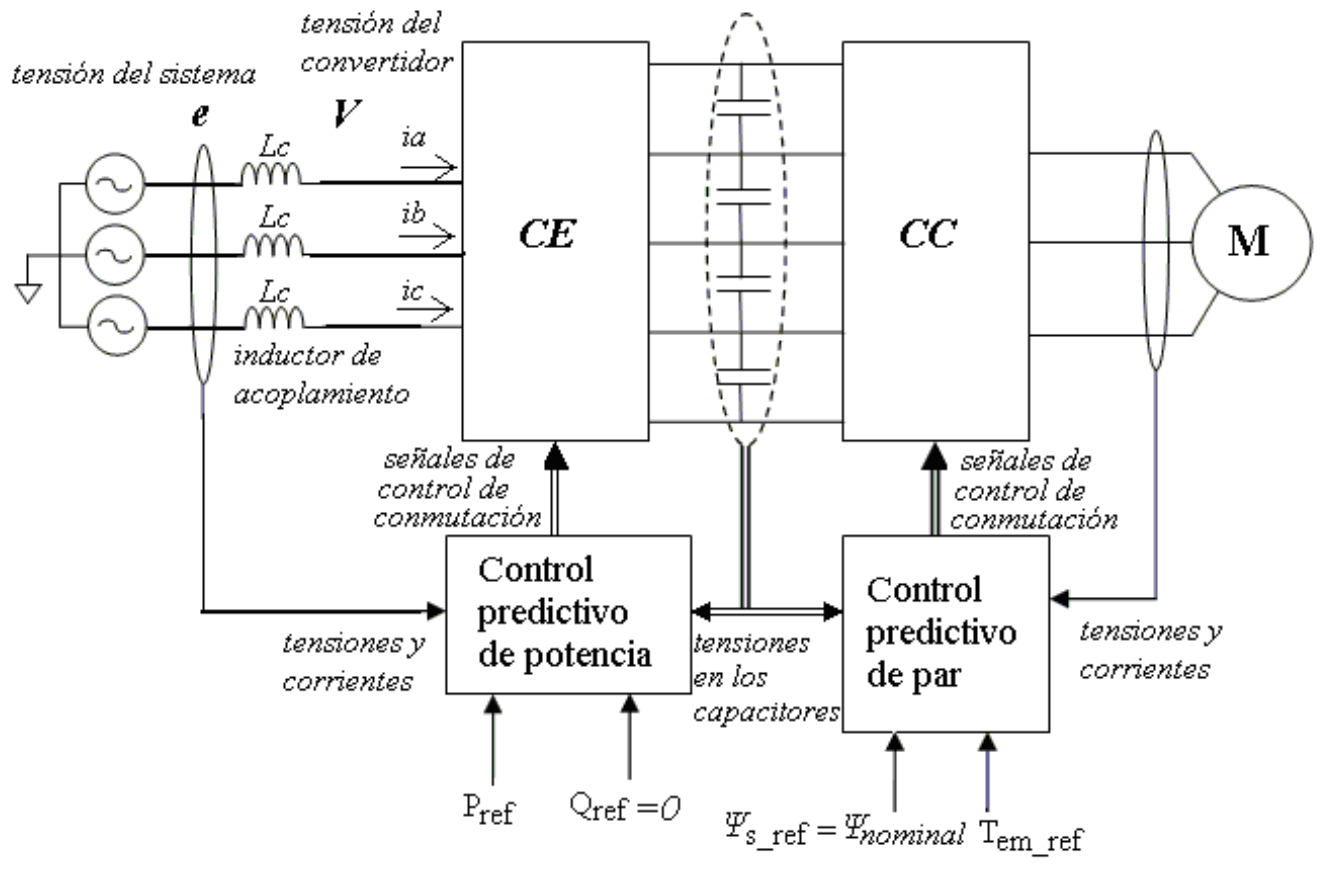

Figura 4.4. Esquema de control del accionamiento basado en la topología back to back $D C M C$.

La estrategia de control en ambos convertidores consiste en el precálculo de las variables de interés en el instante de muestreo $k$ al instante $(k+1)$, y en una comparación de las mismas con los valores de referencia. Estas variables pueden ser clasificadas según estén fuera o dentro del convertidor como externas (corrientes, potencias o variables propias de la carga) o internas (tensiones en los capacitores, número de conmutaciones). Dichas variables son evaluadas para cada combinación de conmutación del convertidor por medio de la función de costo (4.1) que mide la "distancia" entre los valores calculados y los valores de referencia:

$$
g=g_{\text {ext }}+g_{\text {int }}
$$

donde $g_{\text {ext }} \mathrm{y} g_{\text {int }}$ son las funciones de costo asociadas a las variables externas e internas, respectivamente. Para el caso particular de los convertidores de la Fig. 4.3, la función $g_{\text {int }}$ evalúa el estado de balance del bus de continua a fin de mantener equilibradas las tensiones de los capacitores. Esto se logra utilizando el algoritmo explicado en la Sección 3.4.1.2, calculando las tensiones de todos los capacitores a partir de (3.12) y evaluando la función de costo asociada (4.2) $\operatorname{con} N=5$ : 


$$
g_{\text {int }}=g_{V}=\frac{1}{N-1} \sum_{i=1}^{N-1} \frac{\left|V_{\text {Cref }}-V_{C i}[k+1]\right|}{V_{\text {Cref }}}=\frac{1}{4} \sum_{i=1}^{4} \frac{\left|V_{\text {Cref }}-V_{C i}[k+1]\right|}{V_{\text {Cref }}}
$$

Específicamente para el control del $C C$ en la aplicación propuesta, se realiza el control integrado del convertidor de carga y de la máquina mediante la estrategia de control directo de par (DTC) [139][140] adaptada al control predictivo para convertidores multinivel (MPDTC: Model Predictive Direct Torque Control).

La ecuación vectorial del estator del motor de inducción es:

$$
\mathbf{V}_{s}=r_{s} \mathbf{i}_{s}+\frac{d \boldsymbol{\psi}_{s}}{d t}
$$

donde $\mathbf{V}_{\mathrm{s}}, \mathbf{i}_{\mathrm{s}} \mathrm{y} \boldsymbol{\psi}_{\mathrm{s}}$ son la tensión en bobinados del estator, la corriente del estator y el flujo, respectivamente. Considerando que la resistencia $r_{s}$ es pequeña, la Ec. (4.3) se puede aproximar por (4.4):

$$
\Delta \boldsymbol{\psi}_{s} \simeq \mathbf{V}_{s} \Delta t
$$

Esta ecuación indica que en el intervalo de tiempo $\Delta \mathrm{t}$, el vector de flujo sufrirá una variación en el mismo sentido que el vector de tensión estatórico y su módulo variará proporcionalmente al tiempo durante el cual se aplica $\mathbf{V}_{\text {s. }}$ Luego, el módulo de $\boldsymbol{\psi}_{\text {s }}$ puede controlarse mediante la selección de un vector $\mathbf{V}_{\mathrm{s}}$ colineal con él. El conocimiento de $\boldsymbol{\psi}_{\mathrm{s}}$ se logra a través de una estimación a partir de las tensiones y las corrientes en bornes de la máquina. Por otro lado, de acuerdo con [139], bajo condiciones de velocidad del eje y de flujo constantes, dado un incremento en la frecuencia estatórica en $t=0$, el par $E M$ en el eje en ese mismo instante es proporcional a la frecuencia de resbalamiento de la máquina:

$$
\left.\left.T_{e m}\right|_{t=0} \propto \omega_{r e s b}\right|_{t=0}
$$

Esto significa que se puede controlar el aumento o disminución del par motor independientemente de la tasa de variación del módulo del flujo. Luego, el flujo se controla a través de la regulación de la componente radial de la tensión y el par a través de la velocidad de variación de su componente tangencial. De acuerdo con los seis 
movimientos posibles de ambas variables es posible seleccionar el próximo estado del convertidor. La Fig. 4.5 muestra los vectores sintetizables por un convertidor de dos niveles y los seis sectores (denotados con círculos). Cada sector está determinado por un vector activo que es la bisectriz del mismo. La selección del estado del convertidor en un instante dado se realiza mediante la Tabla 4.1.

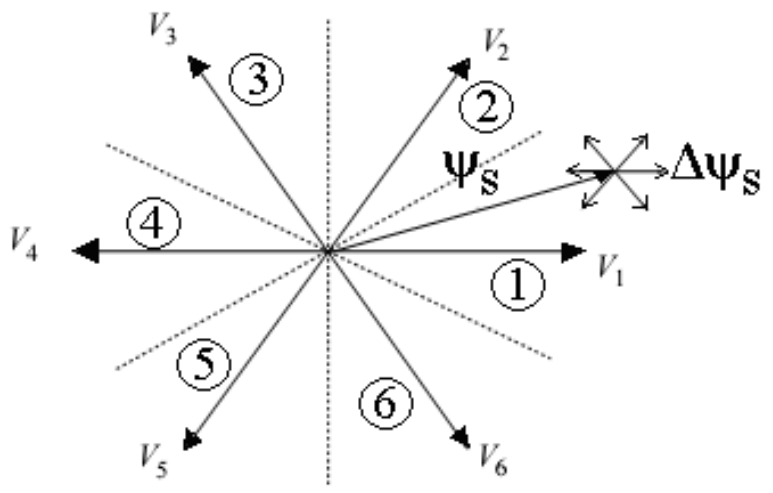

Figura 4.5. Variación de flujo respecto de un punto de equilibrio.

Tabla 4.1. Selección de vectores

\begin{tabular}{|c|c|c|c|c|c|c|c|}
\hline \multirow{2}{*}{$\begin{array}{c}\text { Efecto } \\
\text { deseado }\end{array}$} & \multicolumn{6}{|c|}{ Vector a seleccionar dependiendo del sector } \\
\cline { 2 - 8 } & 1 & 2 & 3 & 4 & 5 & 6 \\
\hline \multirow{3}{*}{$\psi_{s} \uparrow$} & $\mathrm{T}_{\text {em }} \uparrow$ & $V_{2}$ & $V_{3}$ & $V_{4}$ & $V_{5}$ & $V_{6}$ & $V_{1}$ \\
\cline { 2 - 8 } & $\mathrm{T}_{\text {em }}=$ & $V_{7}$ & $V_{0}$ & $V_{7}$ & $V_{0}$ & $V_{7}$ & $V_{0}$ \\
\cline { 2 - 8 } & $\mathrm{T}_{\text {em } \downarrow}$ & $V_{6}$ & $V_{1}$ & $V_{2}$ & $V_{3}$ & $V_{4}$ & $V_{5}$ \\
\hline \multirow{3}{*}{$\psi_{S} \downarrow$} & $\mathrm{T}_{\text {em }} \uparrow$ & $V_{3}$ & $V_{4}$ & $V_{5}$ & $V_{6}$ & $V_{1}$ & $V_{2}$ \\
\cline { 2 - 8 } & $\mathrm{T}_{\text {em }}=$ & $V_{0}$ & $V_{7}$ & $V_{0}$ & $V_{7}$ & $V_{0}$ & $V_{7}$ \\
\cline { 2 - 8 } & $\mathrm{T}_{\mathrm{em}} \downarrow$ & $V_{5}$ & $V_{6}$ & $V_{1}$ & $V_{2}$ & $V_{3}$ & $V_{4}$ \\
\hline
\end{tabular}

Como se observa de la Tabla 4.1, en convertidores de dos niveles, las ecuaciones (4.4) y (4.5) originan una relación directa entre las variaciones posibles de par y flujo y los vectores del convertidor [139]. Sin embargo, la gran cantidad de estados del convertidor multinivel hace que esta selección no sea trivial, razón por la cual la técnica no es directamente extensible y el criterio de selección debe ser más elaborado [141]. En este sentido, la idea ha sido adaptada al control predictivo y se ha denominado MPDTC (Model Predictive Direct Torque Control), reemplazando la Tabla 4.1 por la evaluación de una función de costo que minimiza el error entre el par $\mathrm{y}$ el flujo $[49][110][141][142][143]$. En base a estas consideraciones, sin olvidar la cuestión del balance, se propone como función de costo para el $C C$ la función (4.6): 


$$
g_{C C}=g_{C C \text { ext }}+g_{C C \text { int }}=\left(K_{T} g_{T}+K_{\Psi} g_{\Psi}\right)+\left(K_{V C C} g_{V}\right)
$$

donde $g_{T}$ y $g_{\Psi}$ son las funciones de costo relacionadas con las variables externas (par y flujo), $g_{V}$ evalúa las tensiones en los capacitores (variables internas) y $K$ son los factores de peso correspondientes.

Con respecto a la estrategia de control del convertidor de entrada, se encuentran en la bibliografía esquemas de control de corriente y potencia [47][111][144][145]. El control directo de potencia mediante $F C S-M P C$ posee la ventaja de incorporar en forma explícita la función de control de balance, a la vez que no requiere modulador ni controladores adicionales. En este esquema, la señal de referencia de potencia que debe tomarse de la red de suministro, proviene de la medición o estimación de la potencia consumida por el convertidor de carga y de un término relacionado el estado de carga del bus de continua, donde la interrelación entre la potencia activa y la tensión del bus se resuelve a través del diseño de una señal de referencia de potencia activa consistente [146]. Por lo tanto, esta estrategia permite estabilizar la tensión del bus de continua y actuar sobre la potencia reactiva a fin de presentar a la red un alto factor de potencia. Al igual que el $C C$, el $C E$ tiene en cuenta el balance del bus de continua, llegando a la función (4.7),

$$
g_{C E}=g_{C E e x t}+g_{C E \text { int }}=\left(K_{P} g_{P}+K_{Q} g_{Q}\right)+\left(K_{V C E} g_{V}\right)
$$

Las funciones de costo $g_{P}$ y $g_{Q}$ están asociadas a las potencias activa y reactiva respectivamente (variables externas) y $g_{V}$ tiene el mismo significado que en el caso anterior.

\subsubsection{Convertidor de Carga: control predictivo de par}

El $C C$ y el motor de inducción se controlan en forma conjunta mediante la técnica de control predictivo directo de par. El algoritmo evalúa las componentes propias de control de la máquina (flujo y par) y la función de costo de balance: 


$$
g_{C C}=K_{T} g_{T}+K_{\psi} g_{\psi}+K_{V C C} g_{V}=K_{T} \frac{\left|T_{\text {em_ref }}-T_{\text {em }}[k+1]\right|}{T_{\text {nom }}}+K_{\psi} \frac{\left|\psi_{s_{-} r e f}^{2}-\psi_{s}^{2}[k+1]\right|}{\psi_{\text {ref }}^{2}}+K_{V C C} g_{V}
$$

El vector de flujo magnético depende directamente de las tensiones aplicadas al estator, de acuerdo con (4.9):

$$
\boldsymbol{\psi}_{s}=\int\left(\mathbf{V}_{s}-r_{s} \mathbf{i}_{s}\right) d t
$$

Esta ecuación es definida en el plano $\alpha-\beta$ según (4.10). En la práctica, debido a la deriva de integración se considera el reemplazo de la integración (4.9) por la de un filtro pasabajos con una frecuencia de corte apropiada $\omega_{\text {cutoff. }}$ Expresando las variables en el plano transformado según (4.10) y discretizando (4.9) se deducen las expresiones para el precálculo de las componentes $\alpha$ y $\beta$ del flujo magnético (Ec. 4.11).

$$
\begin{gathered}
{\left[\begin{array}{l}
f_{\alpha} \\
f_{\beta}
\end{array}\right]=\frac{2}{3}\left[\begin{array}{ccc}
1 & -\frac{1}{2} & -\frac{1}{2} \\
0 & \frac{\sqrt{3}}{2} & -\frac{\sqrt{3}}{2}
\end{array}\right]\left[\begin{array}{l}
f_{a} \\
f_{b} \\
f_{c}
\end{array}\right]} \\
\left\{\begin{array}{l}
\psi_{s \alpha}[k+1]=\left(\left(V_{s \alpha}[k]-r_{s} i_{s \alpha}[k]\right) T_{S}+\psi_{s \alpha}[k]\right) \frac{1}{1+T_{S} \omega_{\text {cutoff }}} \\
\psi_{s \beta}[k+1]=\left(\left(V_{s \beta}[k]-r_{s} i_{s \beta}[k]\right) T_{S}+\psi_{s \beta}[k]\right) \frac{1}{1+T_{S} \omega_{\text {cutoff }}}
\end{array}\right.
\end{gathered}
$$

Luego se calcula el módulo del flujo:

$$
\psi_{s}^{2}[k+1]=\psi_{s \alpha}^{2}[k+1]+\psi_{s \beta}^{2}[k+1]
$$

Dichas componentes de flujo permiten, conjuntamente con los valores de corriente en el instante $k$, calcular el par electromagnético mediante (4.13), donde $P_{m}$ es el número de pares de polos de la máquina.

$$
T_{e m}[k+1]=\frac{3}{2} P_{m}\left(\psi_{s \alpha}[k+1] i_{\beta}[k]+\psi_{s \beta}[k+1] i_{\alpha}[k]\right)
$$


La referencia de flujo $\psi_{s_{-} r e f}$ se fija en el valor de flujo nominal y se obtiene de los datos de chapa de la máquina (tensión y frecuencia nominales), mientras que la referencia de par proviene de un lazo de control externo.

\subsubsection{Convertidor de Entrada: control predictivo de potencia}

El objetivo del control del $C E$ es regular la tensión del bus $D C$ con corrientes sinusoidales y factor de potencia unitario. En cada período de muestreo, el algoritmo determina la mejor combinación de conmutaciones del convertidor a fin de drenar de la red de suministro una potencia que satisfaga la demanda de potencia de la carga.

\subsubsection{Cálculo de las corrientes y potencias}

En el instante $k$, se muestrean las tensiones de fase $\mathbf{e}[\mathrm{k}]$ y las corrientes $\mathbf{i}[\mathrm{k}]$. La potencias activa y reactiva instantáneas entregadas por la red en el instante $(k+1)$ pueden ser precalculadas utilizando la teoría de la potencia instantánea a través del cálculo de las corrientes. El mismo se efectúa evaluando (4.14), la cual se obtiene de forma similar a (3.10):

$$
\left[\begin{array}{l}
i_{a} \\
i_{b} \\
i_{c}
\end{array}\right]_{K+1}=\frac{T_{S}}{3 L_{C}}\left(\left[\begin{array}{cc}
2 & 1 \\
-1 & 1 \\
-1 & -2
\end{array}\right]\left(\left[\begin{array}{c}
e_{a b} \\
e_{b c}
\end{array}\right]_{K}-\left[\begin{array}{c}
V_{a b} \\
V_{b c}
\end{array}\right]_{K}\right)\right)+\left(1-\frac{r_{C} T_{S}}{L_{C}}\right)\left[\begin{array}{l}
i_{a} \\
i_{b} \\
i_{c}
\end{array}\right]_{K}
$$

donde $r_{c}$ es la resistencia del inductor de acoplamiento y $e_{x y}, V_{x y}$ las tensiones de línea en el lado del sistema y del convertidor respectivamente (Fig. 4.4). Transformando las variables mediante (4.10) se obtienen las expresiones para la potencia activa y reactiva instantáneas (4.15), considerando que las tensiones del sistema no varían significativamente en un periodo de conmutación.

$$
\begin{aligned}
& P[k+1]=e_{\alpha}[k] i_{\alpha}[k+1]+e_{\beta}[k] i_{\beta}[k+1] \\
& Q[k+1]=e_{\beta}[k] i_{\alpha}[k+1]-e_{\alpha}[k] i_{\beta}[k+1]
\end{aligned}
$$

La expresión (4.15) se evalúa para los estados de conmutación posibles (27, como máximo) definidos por (3.7). Las funciones de optimización para $P$ y $Q$ se diseñan para 
cuantificar la diferencia entre los valores de referencia y los resultados de (4.15). Las funciones individuales se definen en (4.16) para los valores precalculados de $P$ y $Q$, y sus valores de referencia normalizadas a la potencia nominal del convertidor.

$$
g_{P}=\left|\left(\frac{P_{r e f}[k+1]-P[k+1]}{P_{\text {nominal }}}\right)\right| \quad \text { y } \quad g_{Q}=\left|\left(\frac{Q[k+1]}{P_{\text {nominal }}}\right)\right|
$$

La señal $Q_{r e f}$ se iguala a cero para alcanzar corriente en fase con la tensión. La referencia de potencia activa $P_{\text {ref }}$ varía en el tiempo y se conforma usando las ecuaciones de balance energético del sistema teniendo en cuenta la potencia consumida a la salida del inversor y el estado de carga de los capacitores del bus.

\subsubsection{Referencia dinámica de potencia activa}

La potencia necesaria para compensar variaciones de la tensión del bus de continua se determina mediante el análisis del circuito equivalente del bus $D C$ desarrollado en [132], el cual se ilustra en la Fig. 4.6(a).

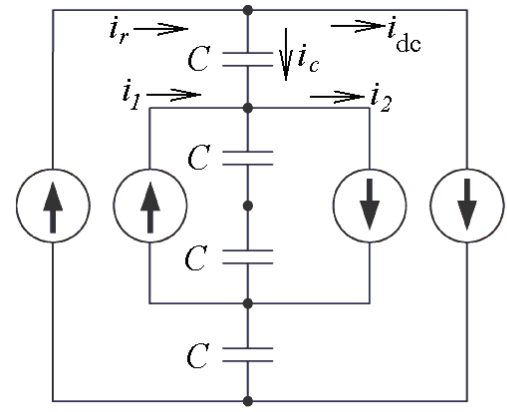

(a)

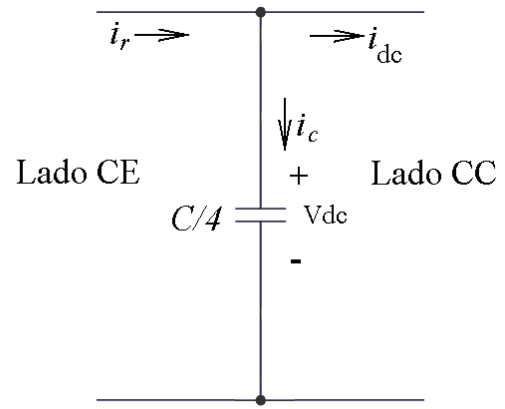

(b)

Figura 4.6. Circuito equivalente del bus de continua.

Considerando que los convertidores operan en condiciones de balance, puede asegurarse que $i_{1}=i_{2}$. En tal caso, la corriente circulante por todos los capacitores tiene el mismo valor $i_{c}$, y el circuito de la Fig. 4.6(a) se reduce a su equivalente de la Fig. 4.6(b). La capacidad equivalente $C_{d c}$ resulta igual a $C / 4$ e $i_{d c}$ representa la corriente drenada por el convertidor de carga. La referencia de potencia para el convertidor de red se compone a partir de la potencia estimada entregada al $C C$ y al bus de continua. La tensión $V_{d c}$ interviene en el cálculo de la potencia activa, como se describe en [146]. Si ocurre una 
desviación en el valor de la tensión del bus $D C$ a partir de su valor de referencia $V_{d c}$ ref la corriente necesaria para compensar el error es:

$$
i_{c_{-} r e f}[k+1]=\frac{C}{4} \frac{1}{T_{S}}\left(V_{d c_{-} r e f}-V_{d c}[k]\right)
$$

donde $T_{S}$ es el periodo de muestreo y $V_{d c}[\mathrm{k}]$ es el valor medido de tensión del bus. Como $i_{d c}$ representa la corriente de carga que consume el $C C$, la corriente $i_{r_{-} r e f}$ que debe entregar el rectificador para suplir ambos requerimientos en el instante $(k+1)$ es entonces:

$$
i_{r_{-} r e f}[k+1]=i_{c_{-} r e f}[k+1]+i_{d c}
$$

La corriente $i_{d c}$ se calcula mediante (4.19) estimando la velocidad del rotor y el par electromagnético de la máquina.

$$
i_{d c}=\frac{P_{e m}}{V_{d c}} \text { donde: } P_{e m}=T_{e m} \omega_{m e c}
$$

La potencia necesaria a ser inyectada en el bus por el $C E$ es $P_{r e f}$ dada por:

$$
P_{r e f}[k+1]=V_{d c} i_{r_{-} r e f}[k+1]
$$

También puede incluirse una protección de sobrecorriente considerando una potencia máxima admisible $P_{\max }$. De esta manera es posible limitar la corriente del convertidor rectificador estableciendo un límite en la señal de referencia de potencia activa de acuerdo con (4.21) (se considera factor de potencia unitario).

$$
P_{r e f}[k+1] \leq P_{\max }=\frac{3 I_{C E \max } \hat{e}}{2}
$$

donde $I_{C E m a x}$ representa la corriente pico máxima admisible a la salida del $C E$ y $\hat{e}$ es la tensión pico nominal de la red de suministro. 
La función de costo completa para el $C E$ (Ec. 4.7) se evalúa para todos los estados posibles de acuerdo con (3.7) y aquella que minimiza la expresión de $g_{C E}$ es seleccionada como el próximo estado del convertidor.

\subsection{Evaluación de la propuesta}

El sistema en estudio consistió en el accionamiento de un motor de inducción de gran potencia en $M T$ alimentado mediante el convertidor B2B DCMC. El diagrama completo se muestra en la Fig. 4.7. Se trató de una máquina asincrónica de $13 k V$ de tensión nominal, el cual fué conectado directamente en bornes del inversor. La tensión del bus fué $20 \mathrm{kV}$ y la carga mecánica consistió en un soplador con una característica de par proporcional al cuadrado de la velocidad. Las variables eléctricas fueron muestreadas para el precálculo de las variables y la evaluación de las funciones de costo. La frecuencia de muestreo se estableció en $10 \mathrm{kHz}$.

Se propusieron cuatro tests para examinar el comportamiento de las variables controladas. La primera prueba consistió en una variación brusca de la carga mecánica aplicada al eje del motor. La segunda prueba consideró un falla en la red que provoca un hueco de tensión a la entrada del convertidor. La tercera prueba comprobó la acción de frenado regenerativo del convertidor y la cuarta demostró la efectividad del algoritmo de balance. 


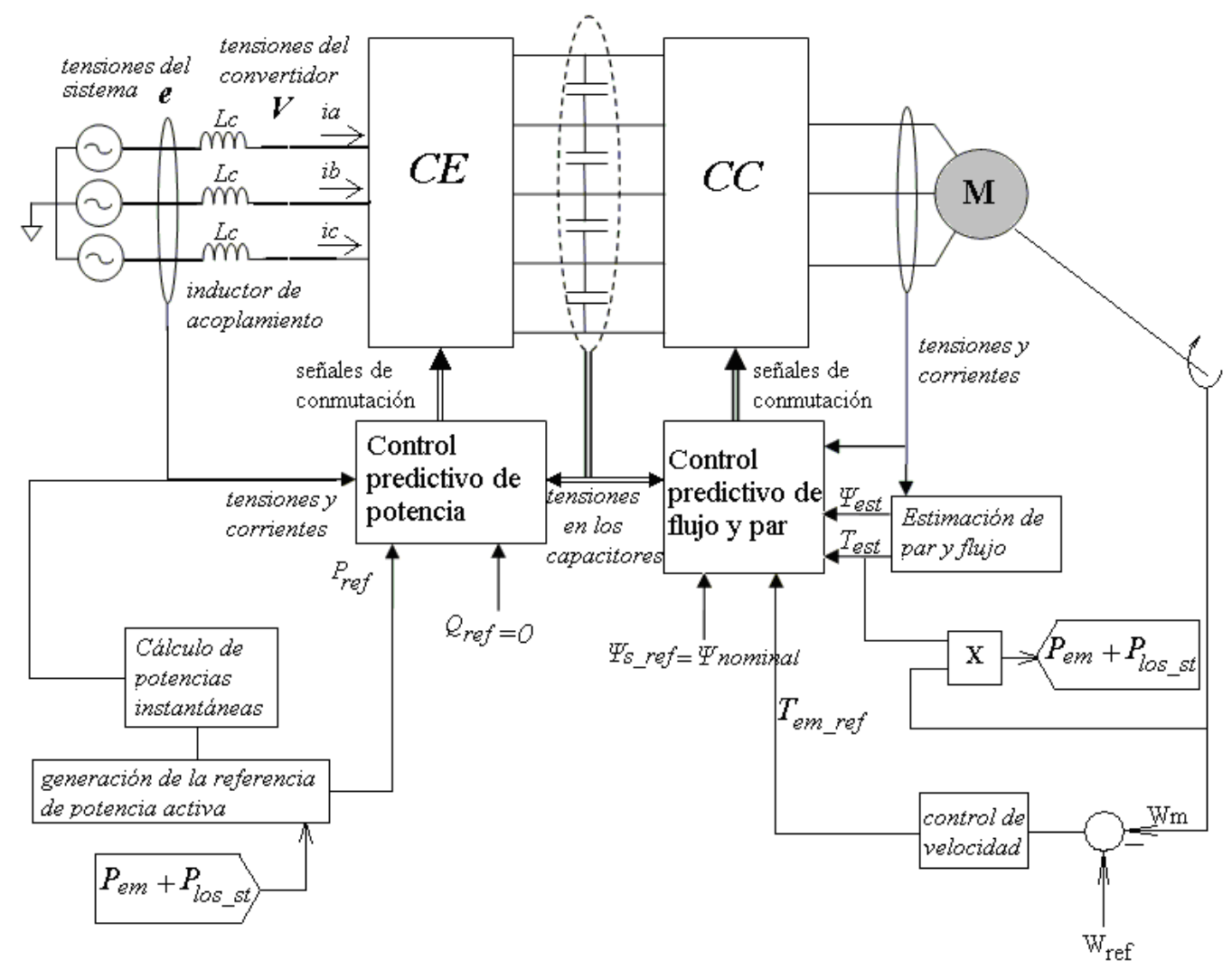

Figura 4.7. Esquema de control del convertidor back to back accionando un motor de inducción.

\section{Variación de la carga mecánica}

La Fig. 4.8 muestra las variables eléctricas del lado del $C E$ cuando se presenta un desacoplamiento súbito de la carga mecánica en el eje del motor en $t=2 \mathrm{~s}$. Dicha carga tomó un valor cercano al nominal $(3600 \mathrm{Nm})$ y decreció a cero en ese instante. El trazo de una tensión de línea de la red y la correspondiente que sintetiza el convertidor aparecen superpuestas en la Fig. 4.8(a). Se observa una variación escalonada con saltos unitarios, aún en el entorno de la perturbación de par sobre el eje, mientras que la conservación del equilibrio de las tensiones del bus $D C$ también se desprende de esta forma de onda ya que no se aprecia pérdida de niveles. Previamente a la perturbación, el bus posee una tensión estable que presenta un pequeño error de estado estacionario, mientras que este valor se aparta en menos del 0,25\% durante el transitorio. El lazo de control tarda alrededor de 40ms en restablecer el valor final de tensión del bus. La corriente drenada de la red es sinusoidal con baja distorsión armónica (Fig. 4.8(c)). La 
Fig. 4.8(d) muestra una tensión de línea del convertidor de carga y una corriente del motor. Se observa una mayor distorsión, en comparación con la tensión sintetizada por el $C E$. Sin embargo, en este caso también se observan transiciones unitarias en la mayor parte del trazo, mientras que la corriente exhibe un perfil sinusoidal cuya amplitud y fase se modifican al momento del desacoplamiento de la carga mecánica. La corriente residual es la componente reactiva de magnetización que mantiene el flujo en su valor nominal independientemente del valor de par en el eje.

La Fig. 4.9(a) muestra la trayectoria descripta por vector de flujo, mientras que la Fig. 4.9(b) ilustra la velocidad de referencia y la medida. Primero, la velocidad se encuentra cerca de su valor de referencia. En este punto la potencia entregada al eje de la máquina es $540 \mathrm{~kW}$ hasta $t=2 \mathrm{~s}$, cuando la carga mecánica es totalmente desacoplada (Fig. 4.9(c)).

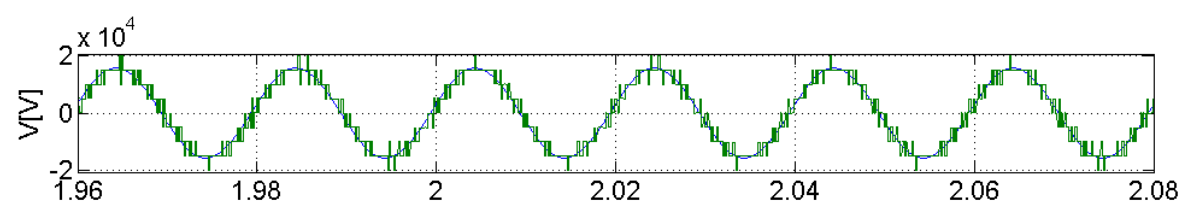

(a)

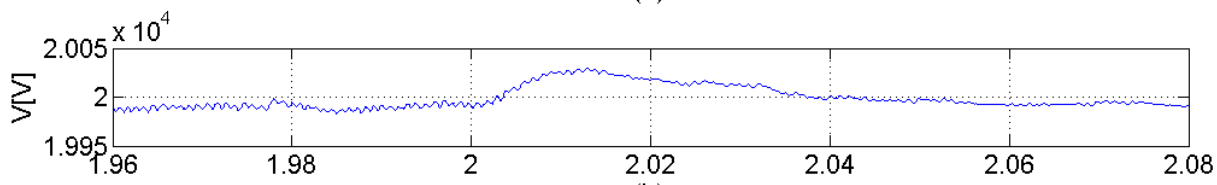

(b)

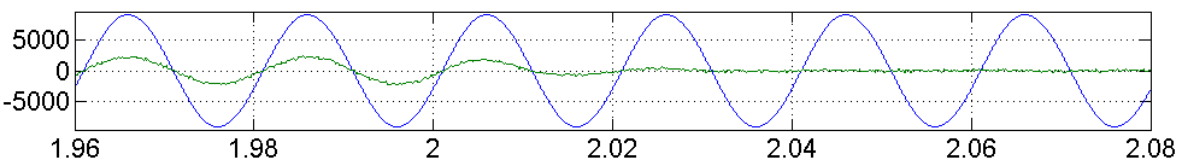

(c)

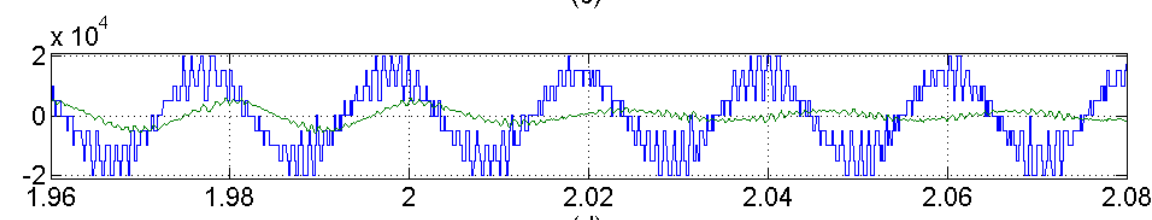

(d)

Figura 4.8. (a) Tensiones de línea a ambos lados del inductor de acoplamiento Lc, (b) Tensión del bus de continua, (c) Tensión de fase y corriente de línea, (d) Tensión de línea y corriente de línea en bornes del motor. 


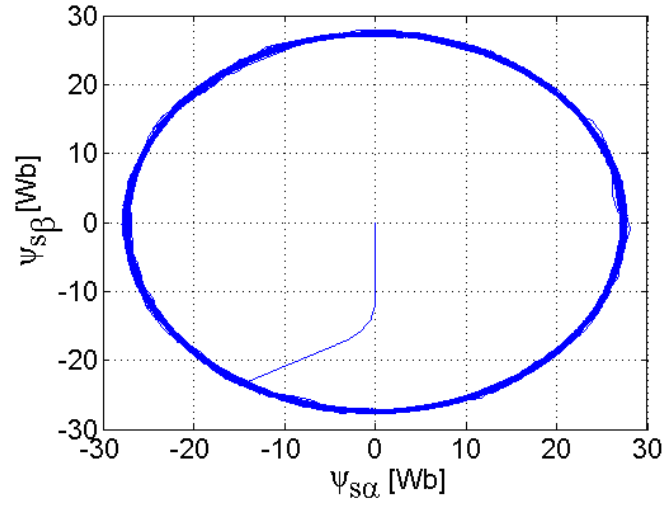

(a)

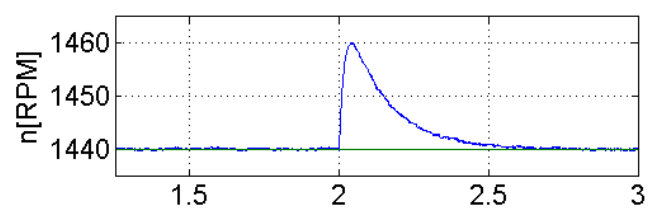

(b)

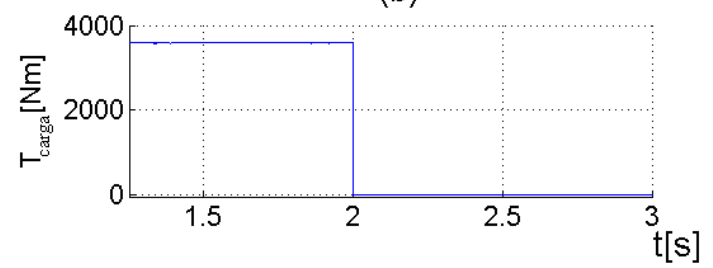

(c)

Figura 4.9. (a) Trayectoria del vector de flujo, (b) Velocidad del eje al momento del cambio de par resistente, (c) Par de carga.

\section{Hueco de tensión}

El segundo test consistió en la reducción de la tensión del sistema. La Fig. 4.10 muestra las tensiones de la red donde un hueco de tensión con una amplitud del $25 \%$ ocurre en $t=2 s$. La Fig. 4.10(a) muestra una tensión de línea a ambos lados del inductor de acoplamiento. Se puede observar la reducción de la componente fundamental de la tensión sintetizada por el convertidor como así también la reducción del número de niveles utilizado para la síntesis. Observando la Fig. 4.10(b), la tensión del bus $D C$ no presenta variación alguna dado que la medición de las tensiones de entrada permiten calcular en forma instantánea la corriente necesaria para mantener el nivel de potencia que debe tomar el $C E$. Una corriente de línea y su correspondiente tensión de fase se presentan en la Fig. 4.10(c), donde es evidente el necesario incremento del valor de corriente para compensar la disminución de la tensión.

La Fig. 4.11 muestra las tensiones sobre los capacitores, las cuales presentan menores desviaciones alrededor del valor de referencia luego de $t=2 \mathrm{~s}$, hecho correspondiente a la mayor disponibilidad de estados de conmutación debido a la disminución del índice de modulación durante el hueco de tensión. 

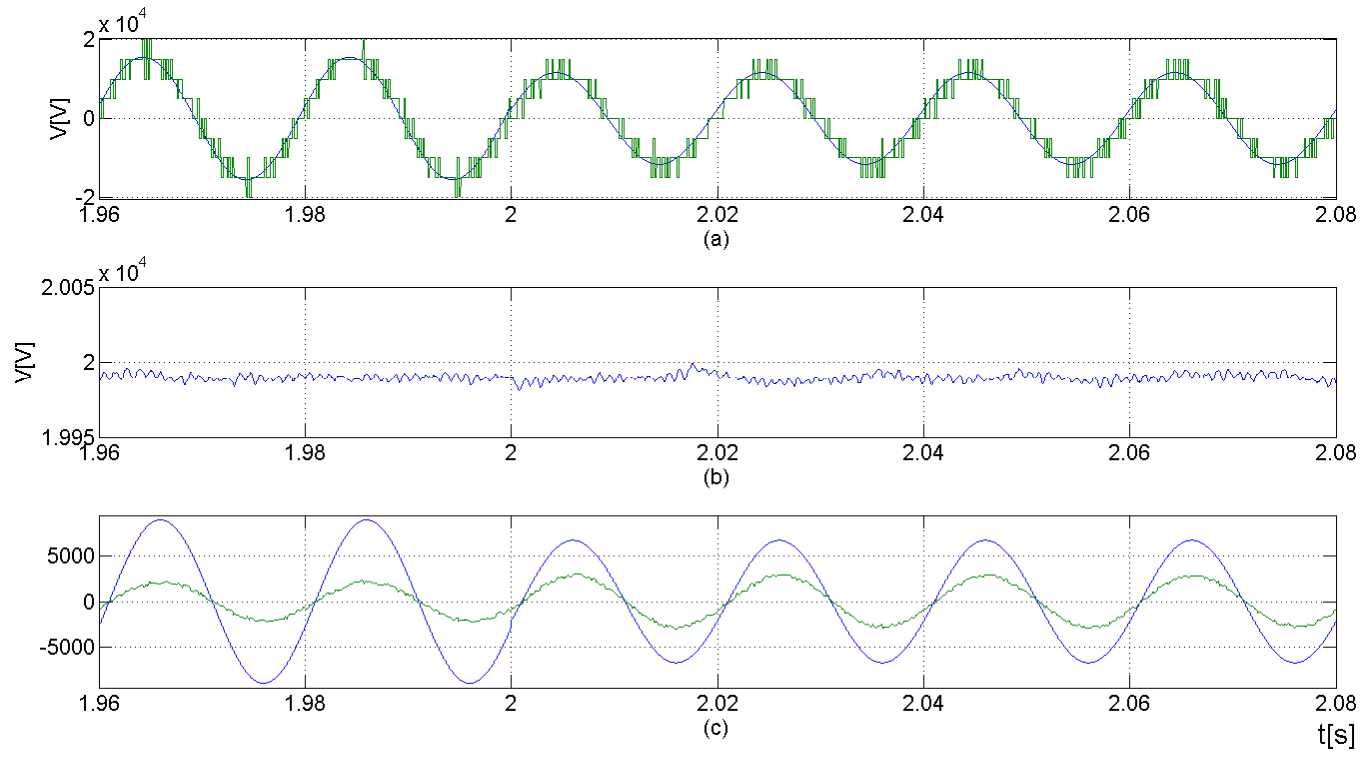

Figura 4.10. (a) Tensiones de línea a ambos lados del inductor de acoplamiento Lc, (b) Tensión del bus de continua, (c) Tensión de fase y corriente de línea correspondiente.

\section{Inversión del flujo de potencia}

Las Figs. 4.12 y 4.13 muestran el resultado de un cambio de signo en la entrada de referencia de par $T_{e m_{-} \text {ref }}$ debido a una disminución del valor de referencia de velocidad a la entrada del control correspondiente. Este cambio se realizó en $t=2 \mathrm{~s}$ e implicó que el motor se comportase como generador devolviendo la energía cinética de la masa rotante hasta alcanzar la nueva velocidad. En la Fig. 4.12 se muestra una tensión de fase de la red y la correspondiente corriente de línea. Se observa el rápido cambio de fase entre ambas pasando del consumo a la generación en un ciclo de red. En la Fig. 4.13 se observa que el balance del bus se preserva también en presencia de esta perturbación. 


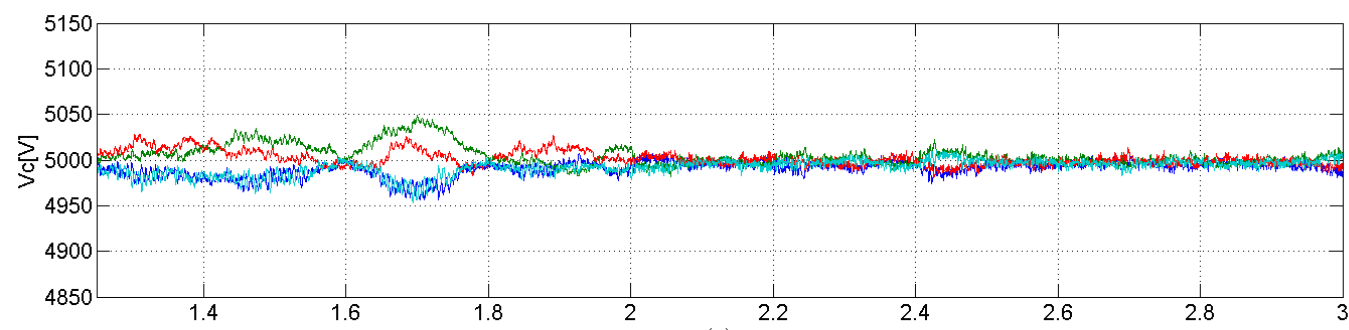

(a)

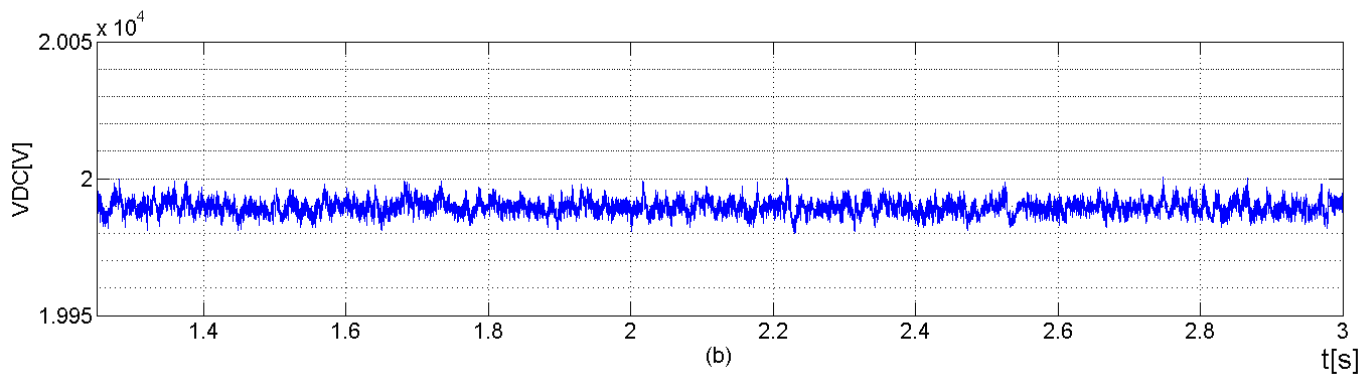

Figura 4.11. (a) Tensiones en los capacitores, (b) Tensión del bus DC.

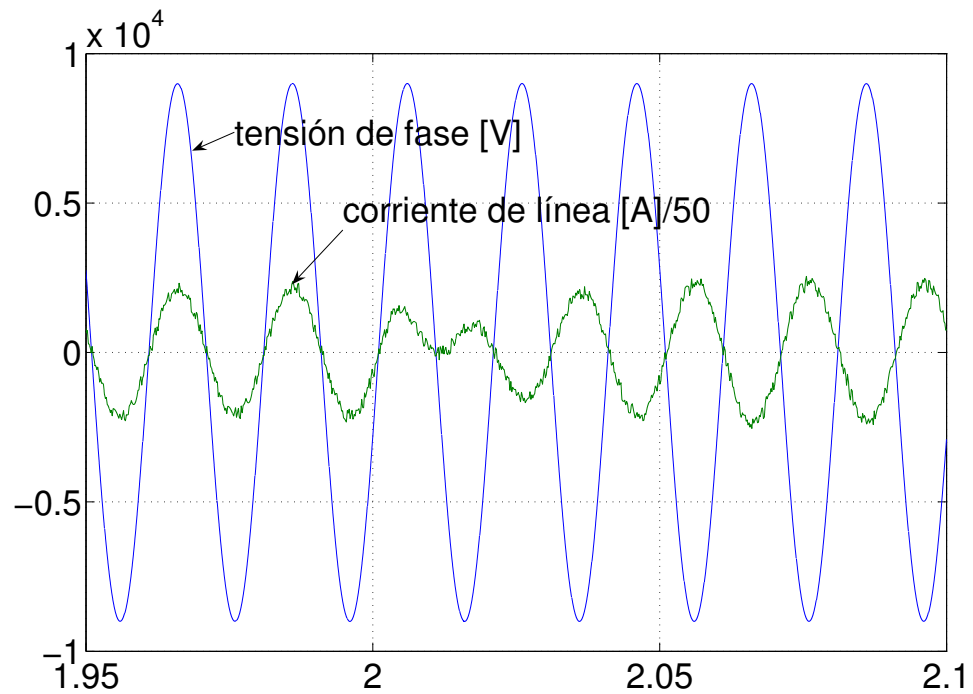

Figura 4.12. Tensión de fase y corriente de línea con frenado regenerativo. 


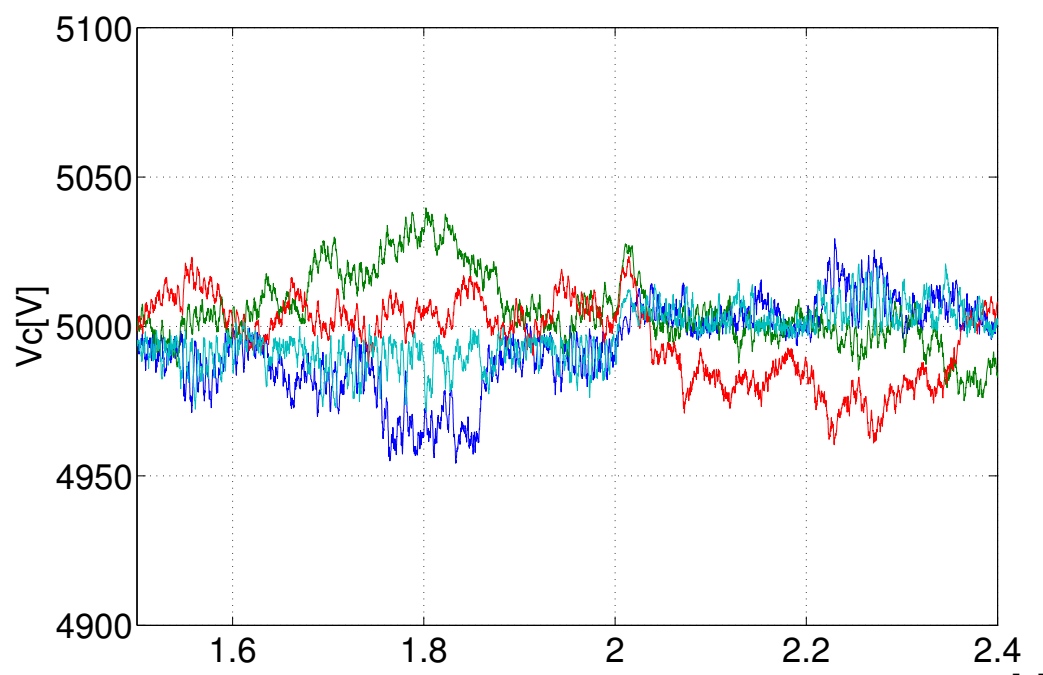

$\mathrm{t}[\mathrm{s}]$

Figura 4.13. Tensiones en los capacitores durante frenado regenerativo en $t=2 s$.

\section{Demostración del Algoritmo de balance y sinergia de carga/descarga}

En este test se pretendió verificar la efectividad de los términos relacionados con el balance de tensiones de los capacitores en sendas funciones de costo $\left(g_{V} \quad \mathrm{y}\right.$ los correspondientes factores de peso $K_{V C E}$ y $K_{V C C}$ ). Para ello se explora su incidencia mediante la anulación de sus valores nominales a partir de un instante dado. Esto implica la desactivación del control de balance del bus, de acuerdo con (4.7), (4.6) y el criterio de minimización explicado en la Sección 4.3.

Inicialmente el sistema se encontraba en el nivel de conversión nominal. Desde $t=1,5 \mathrm{~s}$ a $t=1,65 s, K_{V C E}$ se modificó desde su valor nominal 0,1 a 0 , de manera que se anuló el lazo de realimentación para el balance del bus $D C$ para el $C E$. Se observa de la Fig. 4.14 que los capacitores comienzan a diverger partiendo de sus valores de referencia hasta que se restaura el valor de $K_{V C E}$, momento en el cual retornan a sus valores originales. Similarmente, la Fig. 4.15 muestra el resultado de la misma operación sobre $K_{V C C}$ desde $t=2,25 \mathrm{~s}$ a $t=2,45 \mathrm{~s}$. Al igual que en el caso anterior, el desbalance comienza pero retorna al balance a partir de la restitución del valor de $K_{V C C}$. 


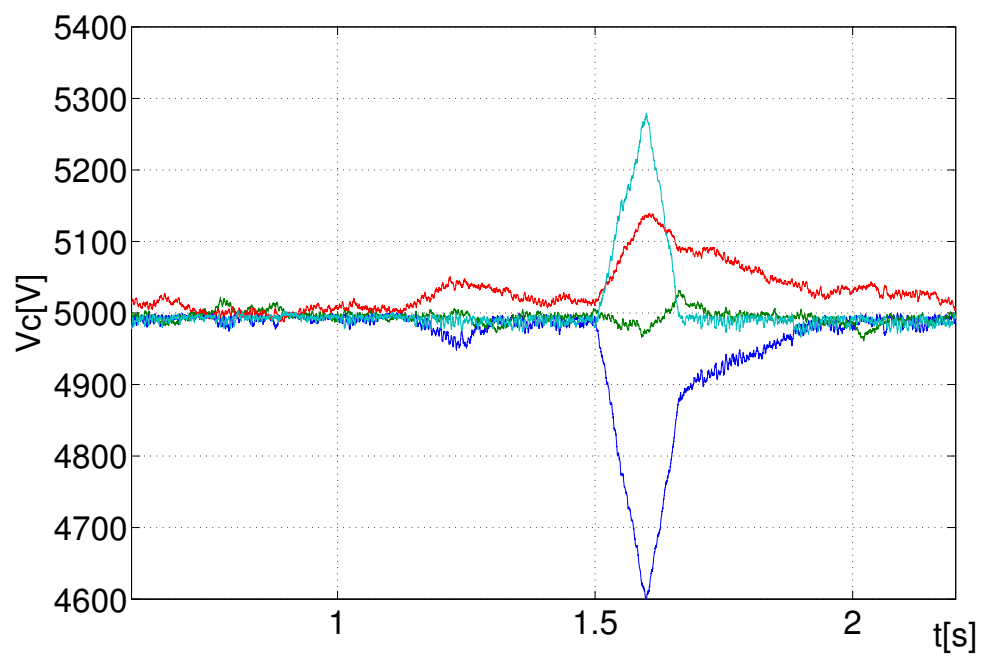

Figura. 4.14. Tensiones en los capacitores durante $K_{V C E}=0$

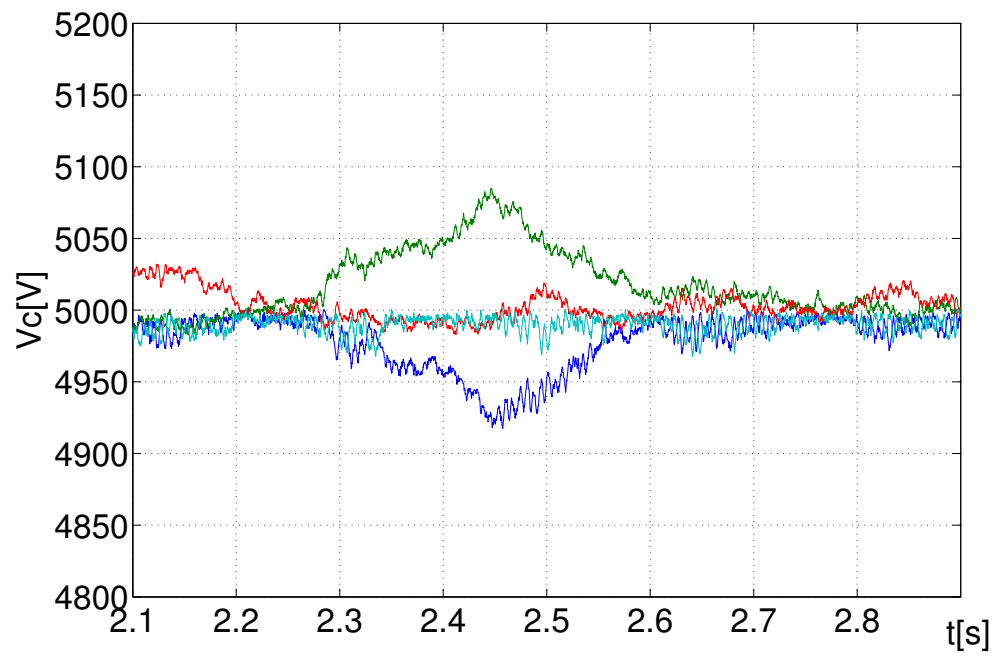

Figura 4.15. Tensiones en los capacitores durante $K_{V C C}=0$

Finalmente, el efecto de compensación de balance debido a la conexión back to back se verifica desconectando los nodos internos del bus y manteniendo $K_{V C C}$ y $K_{V C E}$ en sus valores normales. Los capacitores son divididos en dos bancos separados y asociados a cada convertidor, mientras que una llave de triple contacto con una pequeña resistencia en serie controla el estado de conexión entre ambos convertidores (Fig. 4.16). Primero, la llave se encuentra cerrada y el balance se alcanza normalmente. La llave se abre en $t=2,25 \mathrm{~s}$ y se reconecta en $t=2,30 \mathrm{~s}$. Puede observarse en la Fig. 4.17 que el balance comienza a perderse pero es recuperado y mantenido luego de la reconexión, probando la acción de compensación mutua entre ambos convertidores. También se observa el efecto opuesto de carga/descarga del $C C$ y del $C E$. Los capacitores $C_{2}$ y $C_{3}$ se descargan 
en el lado del $C C$ lo cual constituye el patrón típico para la dirección de flujo de potencia desde el lado $D C$ al $A C\left(P_{D C^{--}}>P_{A C}\right.$, Fig. 2.23(a)), mientras que lo opuesto ocurre con sus contrapartes del lado del $C E\left(P_{A C^{--}}>P_{D C}\right.$, Fig. 2.23(b). Desde luego, la tensión del bus de continua permanece constante debido al control ejecutado por el $C E$, el cual es independiente del algoritmo de balance.
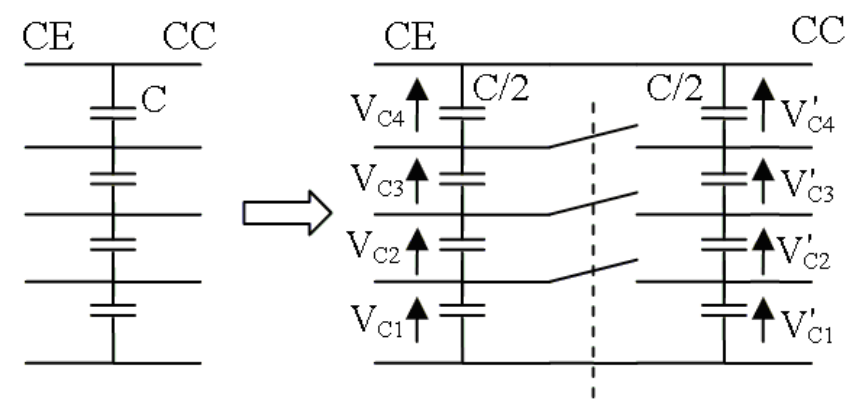

Figura 4.16. Desconexión de los nodos intermedios.

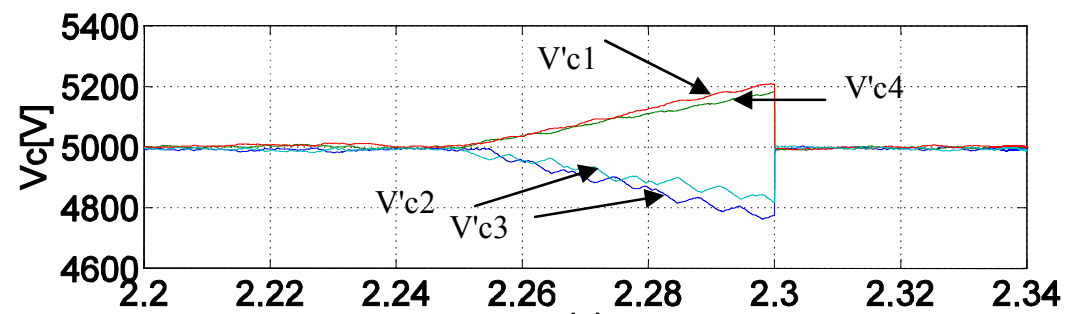

(a)

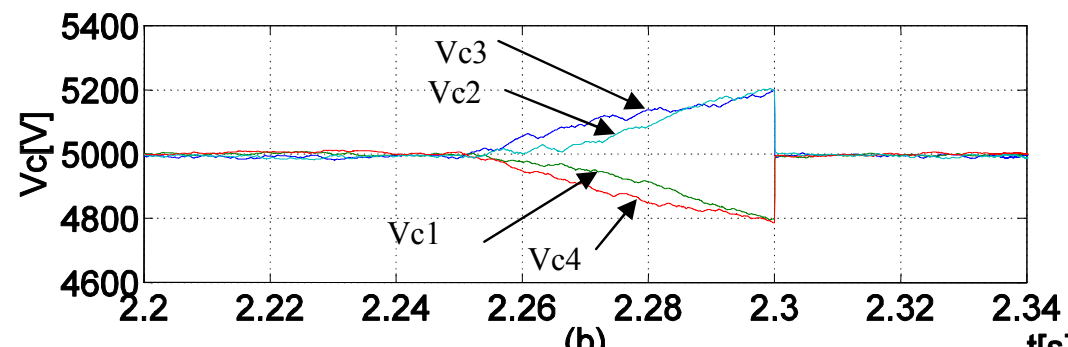

(b) $\mathrm{t}[\mathrm{s}]$

Figura 4.17. Desbalance de los capacitores del bus DC debido a una desconexión transitoria de los nodos intermedios, (a) Lado CC, (b) Lado CE.

\subsection{Conclusiones}

En este capítulo se contextualizó la utilización de convertidores multinivel en accionamientos de alta potencia en $M T$. Se presentó la topología back to back con sus ventajas respecto de sus pares con frente pasivo (especialmente en el rango de altas 
potencias) y se analizó dicha configuración utilizando el convertidor $D C M C$. Se realizó el análisis de compensación de balance del bus de continua y la posibilidad de utilizar dicho algoritmo en forma autónoma a fin de extender la región estable de funcionamiento vista en el Capítulo 2. Se extendió la técnica $F C S-M P C$ vista en el Capítulo 3 para el control del convertidor $B 2 B$. Se desarrolló un control predictivo de potencia para el convertidor de entrada y un control predictivo de par y flujo para el convertidor de carga. El esquema presentado permite la ejecución independiente de las rutinas de control para cada convertidor sin intercambio de información entre convertidores con respecto a sus estados de conmutación. Esto representa un aspecto positivo, en virtud de la carga computacional inherente a esta técnica. Los resultados indican que la configuración cumple con las hipótesis básicas de operación propuestas en la Sección 4.2, ampliando el uso de la topología para otras aplicaciones de conversión de frecuencia o procesamiento de potencia entre dos sistemas de frecuencia diferente. 


\section{Capítulo 5}

\section{Aplicación en Generación Eólica}

\subsection{Introducción}

El incremento en la demanda de energías renovables y la necesidad de disminuir el costo por unidad de energía generada resulta en un incremento del tamaño de las turbinas eólicas. Esto requiere un aumento de las capacidades nominales de todos sus componentes, incluyendo la torre, la maquinaria y los convertidores de potencia. Desde principio de la década de 1980 hasta la actualidad, la potencia de las máquinas ha aumentado dos órdenes de magnitud, encontrándose hoy en día máquinas de serie con potencias de hasta $7.5 \mathrm{MW}[147]$.

Si bien la tecnología basada en la máquina de inducción de rotor bobinado $(D F I G)$ está consolidada en el mercado, los generadores sincrónicos multipolares con excitación independiente (EESG: electrically excited synchronous generator) y en especial los de imanes permanentes (PMSG: permanent magnet synchronous generator) reciben gran interés en la comunidad eólica por permitir la reducción de etapas en la caja multiplicadora y hasta incluso su omisión (direct-drive) [17][21]. Como ventajas particulares el $P M S G$ combina alto índice potencia/peso y carencia de escobillas, lo cual incrementa la robustez, confiabilidad y rendimiento del conjunto [22].

En las turbinas de velocidad variable la velocidad de rotación de las palas y, por ende, la del generador están determinadas por el sistema de seguimiento del punto óptimo. Luego, para una dada velocidad de rotación, la amplitud de la tensión de salida del $P M S G$ es fija debido a la excitación constante de los imanes. Por lo tanto, es preciso convertir la potencia de salida del generador con frecuencia y tensión variables en la 
tensión y frecuencia constantes de la red eléctrica. La adopción de la topología $B 2 B$ permite el control independiente de ambos convertidores con bajo contenido de armónicos de corriente en ambos terminales de alterna. Esto posibilita alcanzar una alta calidad de la potencia entregada a la red y disminuir las pérdidas en el generador, comparando con la topología con rectificador a diodos y convertidor elevador. Como ventaja adicional el convertidor $B 2 B$ separa al generador de la red protegiéndolo ante fallas en la misma.

Otro aspecto de importancia en el diseño de las turbinas son las regulaciones por parte de los operadores de la red eléctrica. Dichas regulaciones se resumen en un conjunto de reglas denominadas códigos de red $(G C)$ que gobiernan la interacción de los generadores con la red de distribución, especialmente en presencia de perturbaciones. Estos requerimientos tienden a brindar confiabilidad a la red interconectada y estabilidad de tensión y frecuencia. A medida que la penetración eólica fue aumentando, las restricciones fueron evolucionando hacia estándares cada vez más exigentes. En este sentido, el cumplimiento de los $G C$ ha sido en gran medida impulsor del desarrollo tecnológico de las turbinas, ya que dichos códigos solo especifican requerimientos en tanto que no se refieren a métodos o tecnologías para alcanzarlos. Estos códigos requieren una alta versatilidad del control de la turbina manteniendo en ambos terminales del sistema (generador y red) una multiplicidad de parámetros en los valores deseados. $\mathrm{Al}$ respecto, puede decirse que la topología con convertidor $B 2 B$ presenta la ventaja de poder inyectar el $100 \%$ de la corriente nominal del equipo ante un requerimiento de inyección de reactivo a la red [148]. Otros parámetros contemplados en los $G C$ son los tiempos de retención ante fallas, mientras que la incorporación de soporte en el mantenimiento de la frecuencia del sistema también es un tema de interés [149][150].

Hasta el momento, la mayoría de las turbinas operan con niveles de baja tensión en el lado del generador $(<690 \mathrm{~V})$. Para niveles de potencia en el orden de los MW, esto implica la circulación de corrientes en el orden de centenares de amperes dentro del generador y a través de los conductores de conexión entre la barquilla y el transformador elevador situado al pie de la torre. Por otra parte, a los efectos de alcanzar dichos valores de corriente es usual la conexión en paralelo de convertidores. Se estima en la actualidad que la migración a niveles de media tensión comienza a ser conveniente cuando las potencias superan los 3MW y en este sentido, los convertidores multinivel con fuente de 
tensión constituyen una alternativa promisoria [23]. Además, con referencia a la interacción de los convertidores con el generador, las ventajas generales en cuanto a la aplicación de formas de onda de tensión escalonada son la disminución de la distorsión de corriente, la disminución de sobretensiones en bornes de la máquina y la prevención de fallas prematuras de rodamientos. También, la circulación de altas corrientes entre el generador y el transformador elevador causa pérdidas por efecto Joule y caída de tensión sobre los cables de conexión. Si bien esto podría resolverse ubicando el transformador en la misma góndola, esta solución no es recomendada debido a restricciones de carga mecánica sobre la torre de la turbina (dicho transformador puede representar el 50-70\% del peso total del convertidor [24][79]). Luego, para combatir este inconveniente se utilizan cables de gran sección, con el consecuente impacto en el costo, y también en la disminución de la capacidad de torsión del conjunto. Esto es importante ya que la barquilla debe soportar varias vueltas de giro para mantener la orientación en la dirección del viento [51]. Este análisis sugiere que la migración a niveles de $M T$ en las turbinas es inevitable. Como antecedentes pueden citarse trabajos sobre aplicación de convertidores multinivel en sistemas eólicos considerando varias topologías [52][53][54], mientras que también se considera el $P M S G$ en conjunto con los $C M$ [22][55][56]. Estas ideas también comienzan a observarse en el ámbito comercial indicando una tendencia clara en esta dirección [57][58][59].

En virtud de lo expuesto se pueden resumir las tendencias de diseño de los equipos de generación eólica de gran potencia en función de las necesidades y los compromisos tecnológicos actuales:

1) Necesidad de aumentar la eficiencia energética. (Velocidad variable, disminución de las pérdidas en todas las etapas de conversión).

2) Necesidad de incrementar la tensión de operación de las turbinas.

3) Necesidad de reducir la cantidad de componentes y el mantenimiento (por ejemplo, mediante la simplificación o la eliminación de la caja de engranajes y de las escobillas).

4) Necesidad de ejecutar los $G C$ con $100 \%$ de la capacidad operativa de la unidad. 
En este capítulo se describe la utilización del convertidor back to back DCMC como interfaz en el camino de potencia de una turbina eólica de velocidad variable en el rango de media tensión sin transformador elevador, la cual está equipada con un generador de imanes permanentes. Asimismo, se explica el desarrollo de un sistema de control para el convertidor del lado de la red a fin de adecuar el comportamiento de la turbina ante fallas en la red eléctrica y cumplir con los GC. El generador, por su parte se controla en conjunto con el convertidor correspondiente mediante la técnica de control predictivo de estados finitos. Los aportes en este tema se publicaron en los artículos [71][72] y [73].

\subsection{Topología con generador de imanes permanentes y convertidor B2B DCMC}

La Fig. 5.1 muestra el diagrama en bloques de un sistema de conversión eólica con generador de imanes permanentes basado en un convertidor $B 2 B D C M C$.

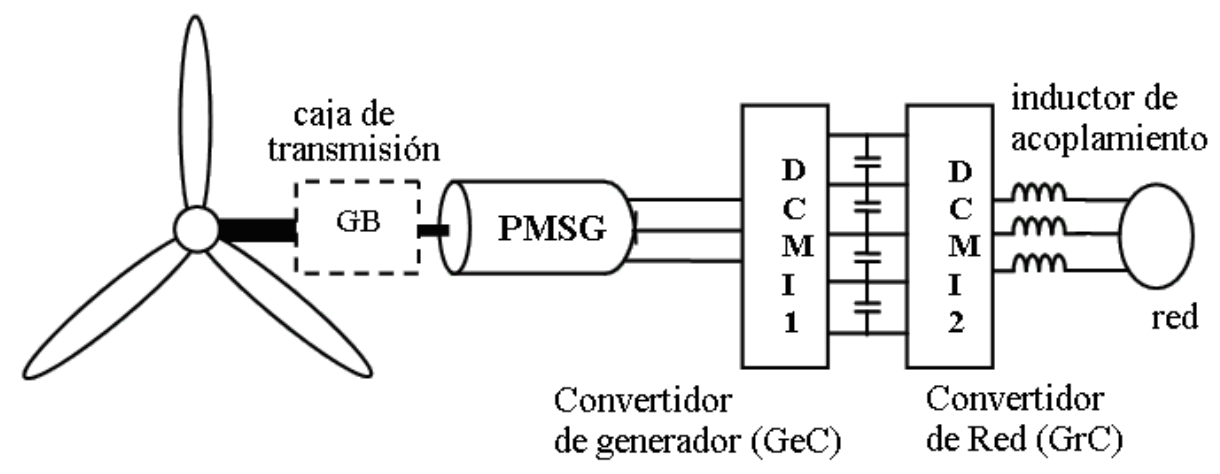

Figura 5.1. Esquema de la turbina y su interfaz de potencia.

Los objetivos de control para el convertidor del lado generador $(\mathrm{GeC})$ y para el convertidor del lado red $(\mathrm{GrC})$ son la optimización del recurso eólico y la conformación de potencia inyectada a la red, respectivamente. El $\mathrm{GeC}$ controla el par de frenado de la máquina para extraer la máxima potencia eólica. Dados la velocidad del viento y el ángulo de pitch, la velocidad de rotación de la turbina para la cual se produce la máxima transferencia de potencia es única. Luego, en condiciones normales de la red eléctrica, la estrategia de control del $\mathrm{GeC}$ define el par resistente a fin de lograr la velocidad de rotación óptima. La potencia extraída del generador es transferida al bus de continua e inyectada a la red a frecuencia y tensión constantes mediante el control del bus $D C$, el cual es ejecutado por el $G r C$. En circunstancias de un hueco de tensión, el $G r C$ inyecta 
corriente reactiva proporcionalmente a la profundidad del hueco a fin de contribuir al mantenimiento de la tensión del sistema.

\subsubsection{Control de los convertidores}

La estructura del convertidor $B 2 B D C M C$ es la presentada en el Capítulo 4 y se repite abreviada en la Fig. 5.2.

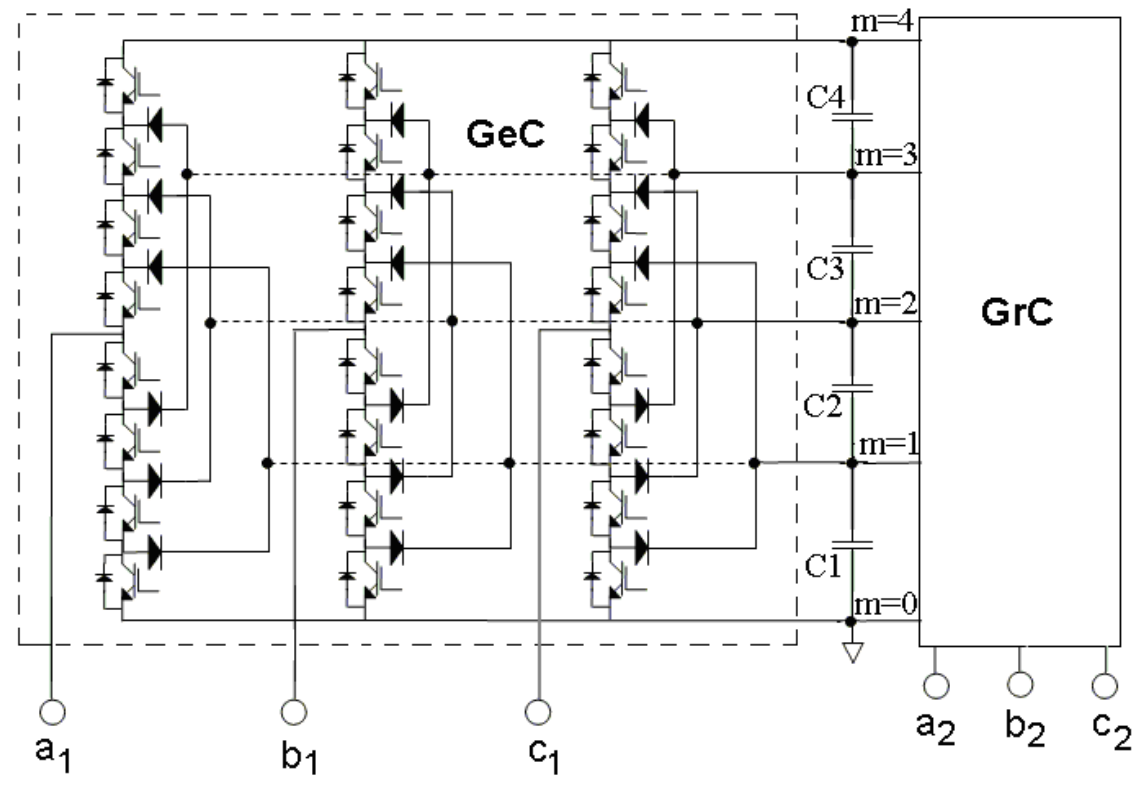

Figura 5.2. Circuito del convertidor DCMC back to back.

Ambos convertidores son controlados mediante el esquema de control predictivo. En este sentido, se ha demostrado que esta técnica también resulta apropiada para el control directo de la máquina de imanes permanentes [112]. Haciendo uso de estas ideas, el $\mathrm{GeC}$ controla la corriente activa del generador $i_{q}$, mientras que la componente en cuadratura $i_{d}$ se fuerza a cero. También se agrega el término de control de balance del bus de continua, indispensable para evitar la pérdida de niveles y preservar la integridad de los dispositivos de conmutación. La función de costo completa tiene la forma (5.1):

$$
g_{G e C}=g_{G e C_{-} e x t}+g_{G e C_{-} \text {int }}=\left(K_{i_{q}} g_{i_{q}}+K_{i_{d}} g_{i_{d}}\right)+\left(K_{V G e C} g_{V}\right)
$$

donde $g_{i q}$ y $g_{i d}$ son las funciones de costo de la corriente activa y reactiva, respectivamente, mientras que $g_{V}$ es la del balance del bus de continua. 
Por otra parte, el control del GrC estabiliza el valor de tensión del bus regulando la potencia activa inyectada a la red. También se controla la potencia reactiva para operar a factor de potencia unitario en condiciones normales de operación o, en presencia de perturbaciones en la tensión de red (huecos de tensión), inyectando corriente reactiva a fin de cumplir con el GC. También se incluye el término de balance en (5.2):

$$
g_{G r C}=g_{G r C_{-} \text {ext }}+g_{G r C_{-} \text {int }}=\left(K_{P} g_{P}+K_{Q} g_{Q}\right)+\left(K_{V G r C} g_{V}\right)
$$

Idénticamente a (5.1), la función $g_{V}$ evalúa el estado de balance del bus de continua mediante la comparación de las tensiones medidas con los valores de referencia según (5.3):

$$
g_{V}=\frac{1}{4} \sum_{i=1}^{4} \frac{\left|V_{\text {Cref }}-V_{C i}[k+1]\right|}{V_{\text {Cref }}}
$$

\subsubsection{Control del generador}

La potencia extraída del tubo de viento incidente está dada por (5.4):

$$
P_{t}=\frac{1}{2} \rho \pi R^{2} V_{W}^{3} C_{P}(\lambda, \beta)
$$

donde $\rho$ es la densidad del aire, $R$ el radio de la turbina, $V_{W}$ la velocidad del viento y $C_{p}$ el coeficiente de potencia. Este coeficiente depende del diseño aerodinámico de las palas, del ángulo de pitch $\beta$ y de la velocidad específica $\lambda$. La Fig. 5.3 muestra esta dependencia en una familia de curvas de $C_{p}$ en función de $\lambda$ con $\beta$ como parámetro. Se desprende de dicha figura que el máximo valor de $C_{p}$ (y en virtud de (5.4)), la máxima potencia de salida) se alcanza cuando $\beta=0$ y $\lambda=\lambda_{\text {opt }}$. Esto se da cuando la velocidad de punta de pala es $\lambda_{\text {opt }}$ veces la velocidad del viento:

$$
\lambda_{\text {opt }}=\frac{\omega_{t_{-} o p t} R}{V_{W}}
$$

Esta expresión indica que para una dada velocidad de viento existe una velocidad de rotación óptima de la turbina $\omega_{\text {t_opt }}$ para la cual la potencia extraída en el eje de la 
máquina es máxima. En estas condiciones, la potencia que puede extraerse y el correspondiente par resistente se expresan en (5.6):

$$
\begin{aligned}
& P_{t_{-} \max }=\frac{1}{2} \rho \pi R^{2} V_{W}^{3} C_{P \max } \\
& T_{t_{-} o p t}=\frac{1}{2} \rho \pi R^{3} \frac{C_{P_{\max }}}{\lambda_{\text {opt }}^{3}} \omega_{t_{-} o p t}^{2}
\end{aligned}
$$

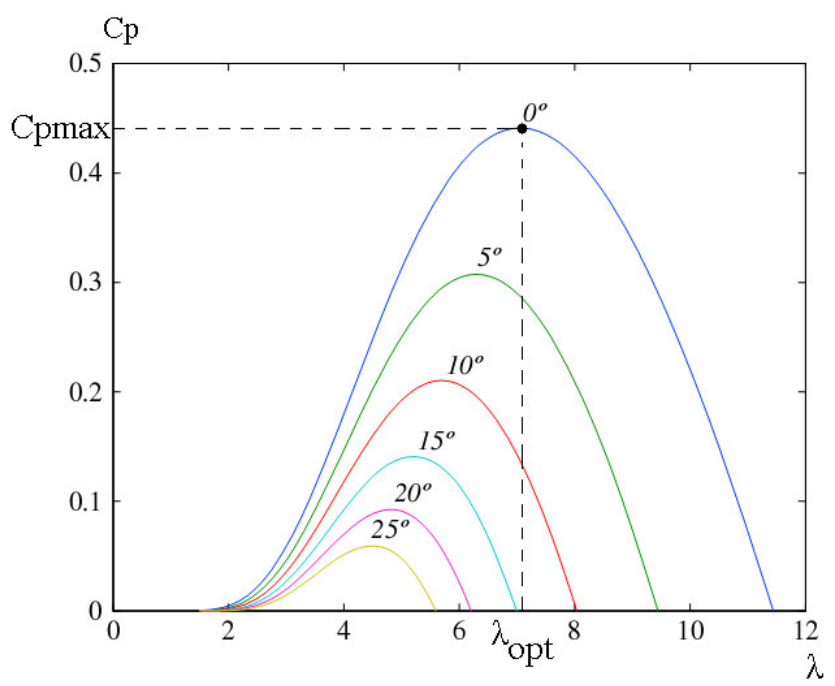

Figura 5.3. Dependencia del coeficiente de potencia con $\lambda$ con ángulo de pitch $\beta$ (en grados) como parámetro.

La Fig. 5.4 muestra las curvas características estáticas de potencia $P_{t}$ de una turbina típica para $\beta=0$ en función de $\omega_{t}$ con $V_{W}$ como parámetro. En la misma figura se muestra la curva correspondiente a la ecuación (5.6(a)) que describe la potencia máxima extraíble en función de la velocidad del rotor de la máquina para un ángulo de pitch $\beta=0$ extendiéndose dicha curva hasta alcanzar la potencia nominal. A partir de este punto, es decir para vientos mayores al nominal, el sistema de control de pitch limita la potencia al valor nominal aumentándolo en la dirección de bandera [151], o bien puede situarse la máquina en un punto de funcionamiento sub-óptimo. 


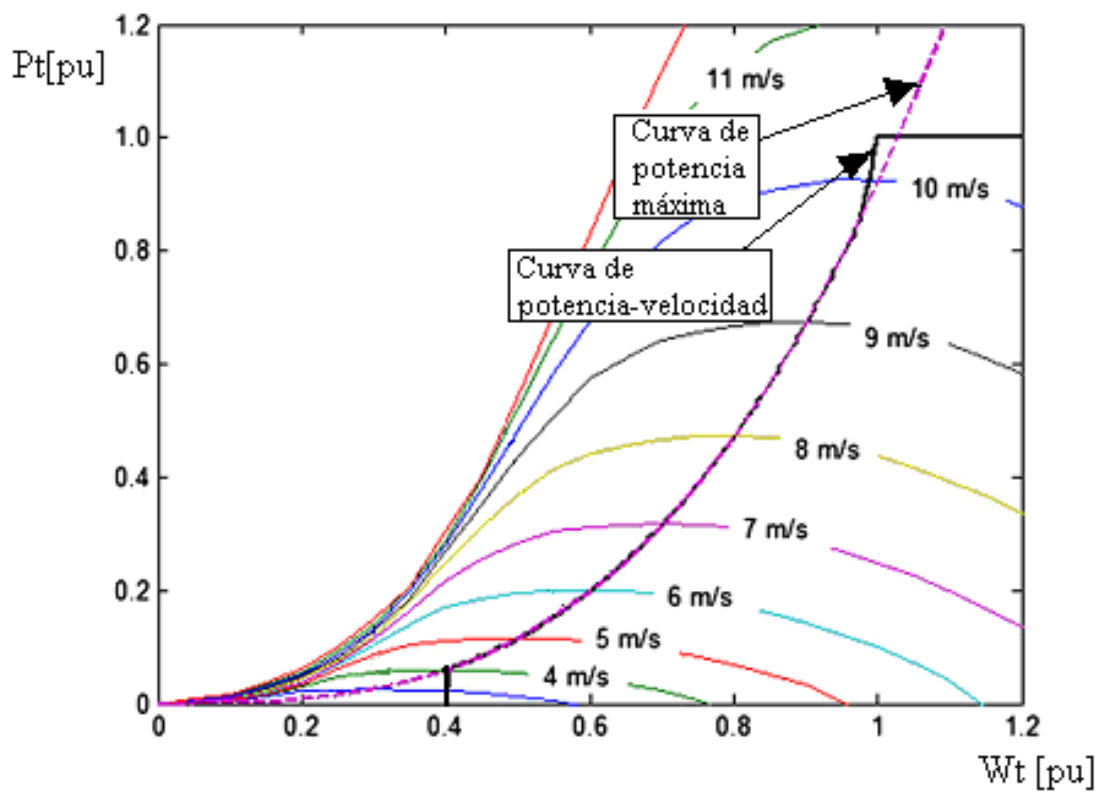

Figura 5.4. Familia de curvas de $P_{t}$ en función de la velocidad de giro de la turbina (velocidad del viento como parámetro).

La Fig. 5.5 muestra un diagrama completo del control del GeC. El lazo de control externo consiste en la regulación de la velocidad de rotación del eje principal $\omega_{t}$, forzando a la máquina a operar en el punto de velocidad específica óptima $\lambda_{\text {opt }}$ ejerciendo sobre el eje de la misma un par de frenado adecuado. El $\mathrm{GeC}$ debe asegurar las corrientes del generador para ajustar el par resistente al valor óptimo (5.6(b)) mediante la síntesis correcta de las combinaciones de conmutación.

Las tensiones de fase sobre los bobinados del generador se calculan en base a las tensiones de línea del convertidor mediante (5.7):

$$
\mathbf{V}=\left[\begin{array}{l}
V_{a} \\
V_{b} \\
V_{c}
\end{array}\right]=\frac{1}{3}\left[\begin{array}{cc}
2 & 1 \\
-1 & 1 \\
-1 & -2
\end{array}\right]\left[\begin{array}{c}
V_{a b} \\
V_{b c}
\end{array}\right]
$$




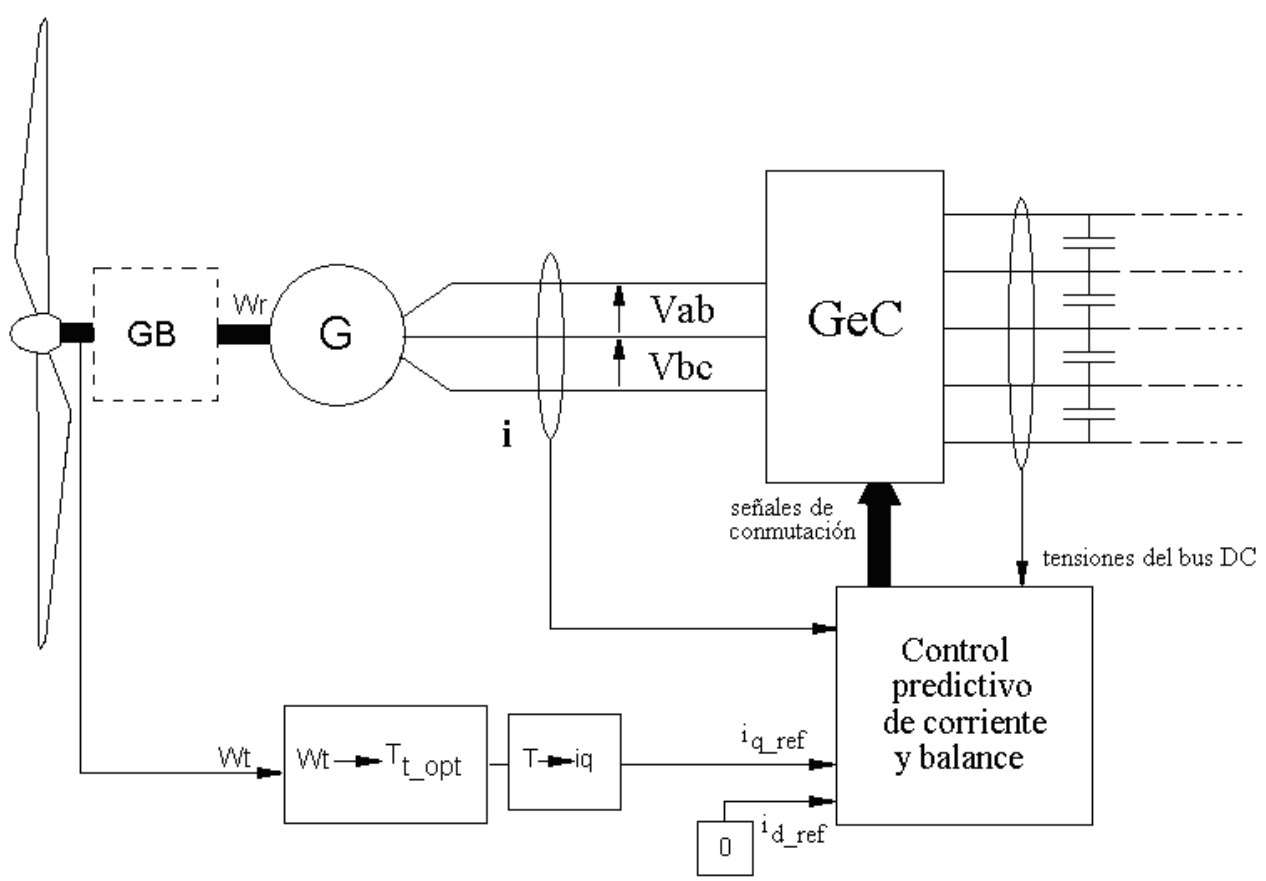

Figura 5.5. Diagrama de control externo del GeC.

La ecuación vectorial del $P M S G$ [112] es:

$$
\mathbf{V}=R_{s} \mathbf{i}+\frac{d \boldsymbol{\psi}_{s}}{d t}
$$

donde i es la corriente de línea, $R_{s}$ la resistencia estatórica y $\psi_{s}$ el flujo magnético que enlaza los devanados del estator, que a su vez depende del flujo producido por los imanes $\left(\psi_{m}\right)$, la orientación del rotor $\left(\theta_{r}\right)$ y la inductancia $\left(L_{s}\right)$ y la corriente (i) del estator:

$$
\boldsymbol{\psi}_{s}=L_{s} \mathbf{i}+\psi_{m} e^{j \theta_{r}}
$$

Transformando al marco sincrónico $d-q$ se llega a las expresiones (5.10(a)) y (5.10(b)), donde $P_{g}$ representa el número de polos del generador. La ecuación $\mathbf{( 5 . 1 0 ( c ) ) ~ e x p r e s a ~ e l ~}$ par electromagnético ejercido sobre el rotor de la máquina. 


$$
\left\{\begin{array}{l}
\frac{d i_{d}}{d t}=\frac{1}{L_{d}} V_{d}-\frac{R_{s}}{L_{d}} i_{d}+\frac{L_{q}}{L_{d}} P_{g} \omega_{r} i_{q} \\
\frac{d i_{q}}{d t}=\frac{1}{L_{q}} V_{q}-\frac{R_{s}}{L_{q}} i_{q}-\frac{L_{d}}{L_{q}} P_{g} \omega_{r} i_{d}-\frac{\psi_{m} P_{g} \omega_{r}}{L_{q}} \\
T_{e m}=\frac{3}{2} P_{g} \psi_{m} i_{q}
\end{array}\right.
$$

Discretizando (5.10(a)) y (5.10(b)) mediante la aproximación de Euler se llega a (5.11):

$$
\begin{aligned}
& i_{d}[k+1]=T_{S}\left(\frac{1}{L_{d}} V_{d}[k]-\frac{R_{s}}{L_{d}} i_{d}[k]+\frac{L_{q}}{L_{d}} P_{g} \omega_{r} i_{q}[k]\right)+i_{d}[k] \\
& i_{q}[k+1]=T_{S}\left(\frac{1}{L_{q}} V_{q}[k]-\frac{R_{s}}{L_{q}} i_{q}[k]-\frac{L_{d}}{L_{q}} P_{g} \omega_{r} i_{d}[k]-\frac{\psi_{m} P_{g} \omega_{r}}{L_{q}}\right)+i_{q}[k]
\end{aligned}
$$

Ambas ecuaciones se evalúan a partir de los estados posibles del convertidor expresados en (3.7). Las funciones de costo para la evaluación de ambas corrientes se expresan en (5.12).

$$
g_{i_{d}}=\frac{\left|i_{d_{-} r e f}-i_{d}[k+1]\right|}{i_{\text {nom }}} \quad \mathrm{y} \quad g_{i_{q}}=\frac{\left|i_{q_{-} r e f}-i_{q}[k+1]\right|}{i_{\text {nom }}}
$$

donde $i_{d_{-} \text {ref }}$ y $i_{q_{-} \text {ref }}$ son las corrientes de referencia. El objetivo de control consiste en lograr en el eje de la máquina un par en oposición al de la turbina (objetivo de $i_{q_{-}}$ref) y a la vez evitar la circulación de corrientes que no produzcan par. En consecuencia, considerando en forma general la existencia de una caja de engranajes de relación $K_{g b}$ entre la turbina y el generador, las referencias de corriente pueden sintetizarse en (5.13).

$$
\begin{aligned}
& i_{d_{-} \text {ref }}=0 \\
& i_{q_{-} \text {ref }}=\frac{2}{3 P_{g} \psi_{m}} \frac{T_{t_{-} o p t}}{K_{g b}}
\end{aligned}
$$

La combinación de conmutación que minimiza (5.1) se selecciona como próximo estado del convertidor. 


\subsubsection{Control del convertidor de red}

La Fig. 5.6 muestra el esquema de control completo del GrC. El convertidor de red controla las potencias activa y reactiva instantáneas inyectadas a la red mediante el algoritmo predictivo para todas las combinaciones de conmutación posibles. El algoritmo predictivo calcula los valores futuros de las corrientes de línea mediante (5.14).

$$
\left[\begin{array}{l}
i_{a} \\
i_{b} \\
i_{c}
\end{array}\right]_{K+1}=\frac{T_{S}}{3 L_{C}}\left(\left[\begin{array}{cc}
-2 & -1 \\
1 & -1 \\
1 & 2
\end{array}\right]\left(\left[\begin{array}{l}
V_{a b} \\
V_{b c}
\end{array}\right]_{K}-\left[\begin{array}{l}
e_{a b} \\
e_{b c}
\end{array}\right]_{K}\right)\right)+\left(1-\frac{r_{C} T_{S}}{L_{C}}\right)\left[\begin{array}{l}
i_{a} \\
i_{b} \\
i_{c}
\end{array}\right]_{K}
$$

Utilizando la transformación estacionaria, las potencias instantáneas se calculan mediante (5.15). Se calculan dichas ecuaciones como máximo para un conjunto de 27 valores de potencia activa y reactiva correspondientes a cada uno de los estados de conmutación admitidos por (3.7).

$$
\begin{aligned}
& P[k+1]=e_{\alpha}[k] i_{\alpha}[k+1]+e_{\beta}[k] i_{\beta}[k+1] \\
& Q[k+1]=e_{\beta}[k] i_{\alpha}[k+1]-e_{\alpha}[k] i_{\beta}[k+1]
\end{aligned}
$$

Las funciones de costo asociadas con $P$ y $Q$ indican el error de dichas variables respecto de sus valores de referencia. Dichas funciones se expresan en (5.16) normalizadas al valor de potencia nominal de la turbina.

$$
g_{P}=\left|\left(\frac{P_{r e f}[k+1]-P[k+1]}{P_{\text {nom }}}\right)\right| \quad, \quad g_{Q}=\left|\left(\frac{Q_{\text {ref }}[k+1]-Q[k+1]}{P_{\text {nom }}}\right)\right|
$$

Las señales de referencia $P_{r e f}$ y $Q_{r e f}$ para el control predictivo son administradas por el bloque de conformación de consignas de potencia activa y reactiva, $P_{r e f}$ y $Q_{r e f}$. La referencia de potencia activa $P_{\text {ref }}$ que debe inyectarse a la red utiliza las ecuaciones de balance energético entre la potencia generada y la energía acumulada en el bus de continua, similarmente a lo explicado en la Sección 4.3.2.2. 


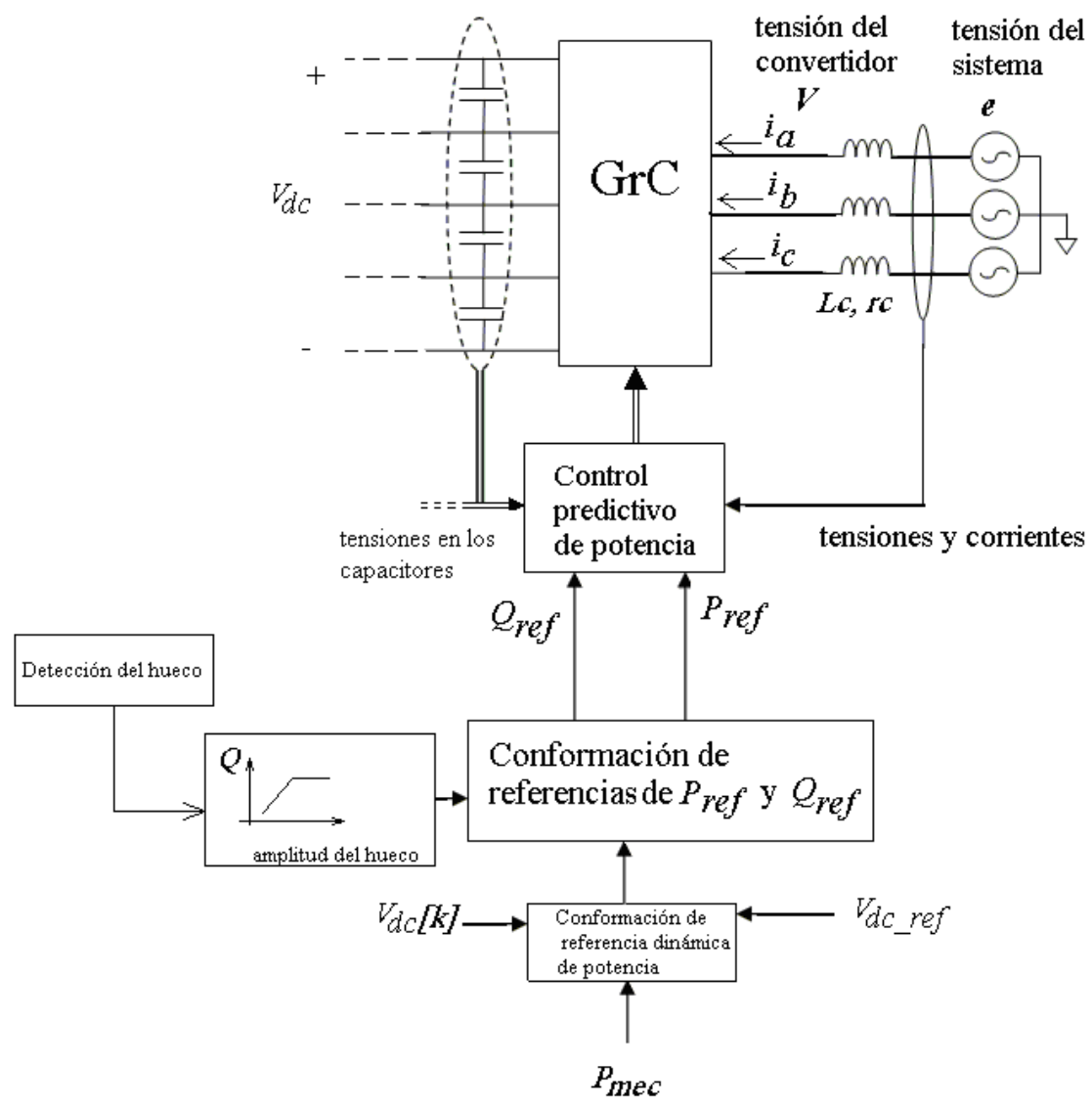

Figura 5.6. Control externo de potencia activa y reactiva.

Por otra parte, la referencia de potencia reactiva $\left(Q_{\text {ref }}\right)$ se establece a partir del modo de operación vigente (modo normal o modo de falla). Si la tensión del sistema se encuentra dentro de la banda de $+-10 \%$ de su valor nominal (funcionamiento normal) ésta se establece en cero, de modo que el convertidor trabaje a factor de potencia unitario. Si ocurre un hueco de tensión, el control pasa a modo de falla y el valor de $Q_{\text {ref }}$ se determina en función de la amplitud del hueco, a partir de lo que se establece en la Fig. 5.7(b) [152]. La misma figura también especifica las restricciones con respecto al tiempo de retención de conexión mínimo. La Fig. 5.7(a) muestra la zona de desconexión admitida dependiendo del tiempo de duración de la falla y de la amplitud del hueco. 


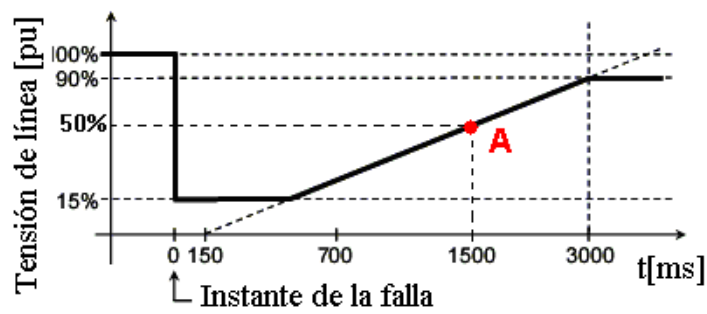

(a)

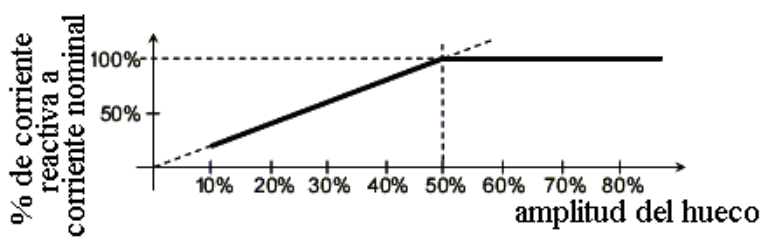

(b)

Figura 5.7. Representación grafica del GC, (a) Tiempo de retención mínimo en función del tiempo de duración del hueco, (b) Corriente reactiva en función de la amplitud del hueco.

El diagrama del control se muestra en la Fig. 5.8. El administrador de potencia determina las señales de referencia a ser procesadas por ambos algoritmos predictivos dependiendo del estado de la red (estado normal o en falla). Cuando el nivel de tensión se encuentra dentro de la banda de tolerancia, el $\mathrm{GeC}$ carga al generador para extraer potencia máxima, la cual es inyectada a la red por el $\mathrm{GrC}$ con factor de potencia unitario y frecuencia constante. En cambio, si existe un hueco de tensión, se provee corriente reactiva en forma proporcional a la amplitud del hueco, llegando al 100\% de la corriente nominal de la turbina para una profundidad de hueco del 50\% (Fig. 5.7(b)). En este caso la potencia activa inyectada a la red debe disminuir a medida que la reactiva aumenta a fin de no exceder la corriente nominal del convertidor $\operatorname{GrC}\left(i_{\text {nom }}\right)$. 


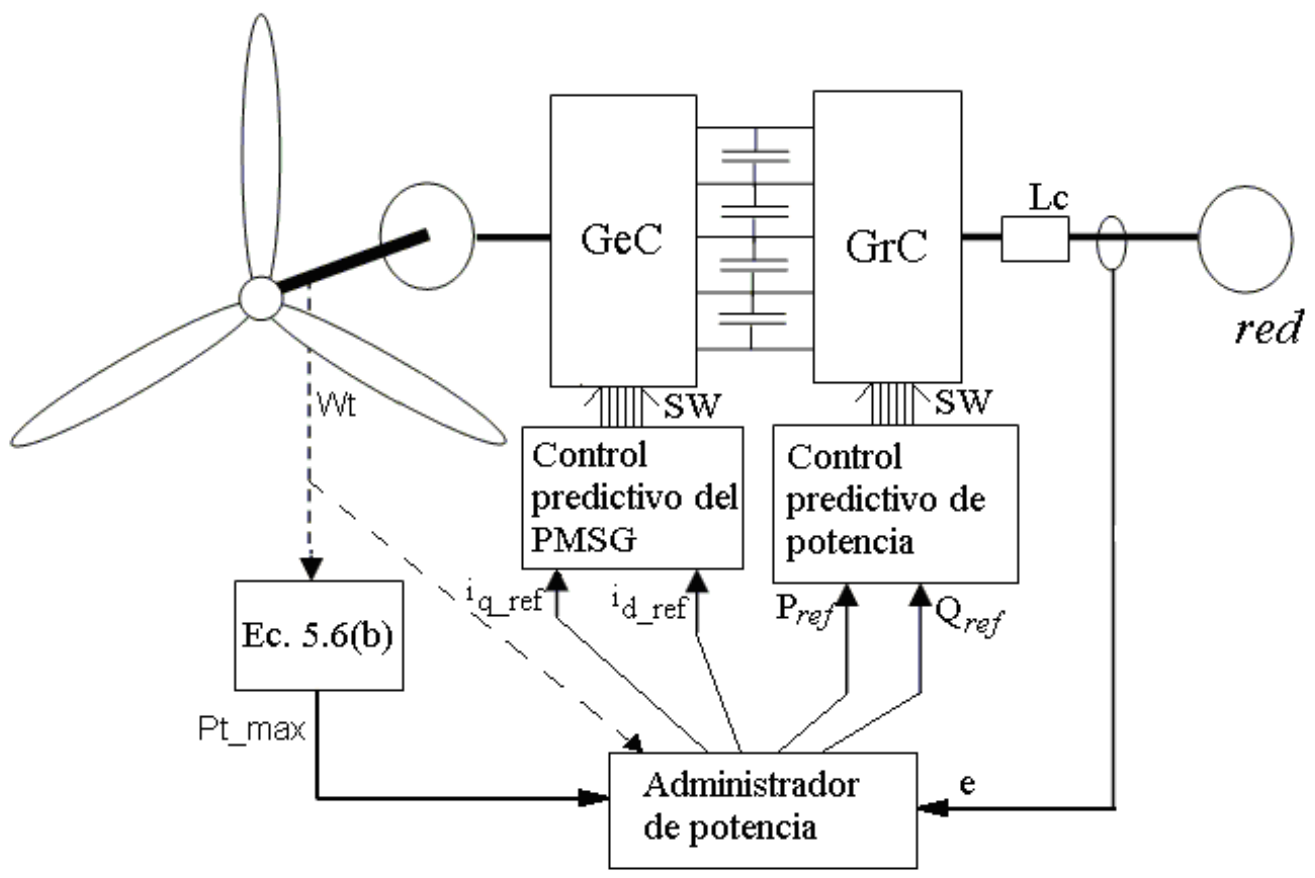

Figura 5.8. Diagrama del control de potencia.

El esquema previsto para la administración de potencia activa y reactiva se detalla en la Fig. 5.9. La tensión $e$ del sistema se monitorea, verificándose su valor dentro de la banda de tolerancia. En función de la amplitud del hueco se calcula el valor de corriente reactiva correspondiente (Fig. 5.7(b)). Este valor es multiplicado por la tensión del sistema para dar como resultado la potencia reactiva que es preciso inyectar a la red (si $e$ se encuentra dentro del $10 \% i_{\text {react }}=0$ y en consecuencia $Q_{r e f}=0$ ). Para expresar la capacidad operativa máxima $S_{\max }($ en $V A$ ) en presencia del hueco, se multiplican la corriente máxima del mismo $i_{\max } \mathrm{y}$ la tensión medida $e$. Luego con $Q_{r e f} \mathrm{y} S_{\max }$ se calcula la potencia activa residual $P_{\text {sat }}$ que es posible extraer de la turbina sin sobrepasar el límite de corriente del convertidor. $P_{\text {sat }}$ será el valor residual de potencia activa extraíble de la turbina, el cual es enviado directamente como señal de referencia de potencia a inyectar en la red. Además, este valor conforma, a través de (5.13), la referencia de par de frenado para el generador. 


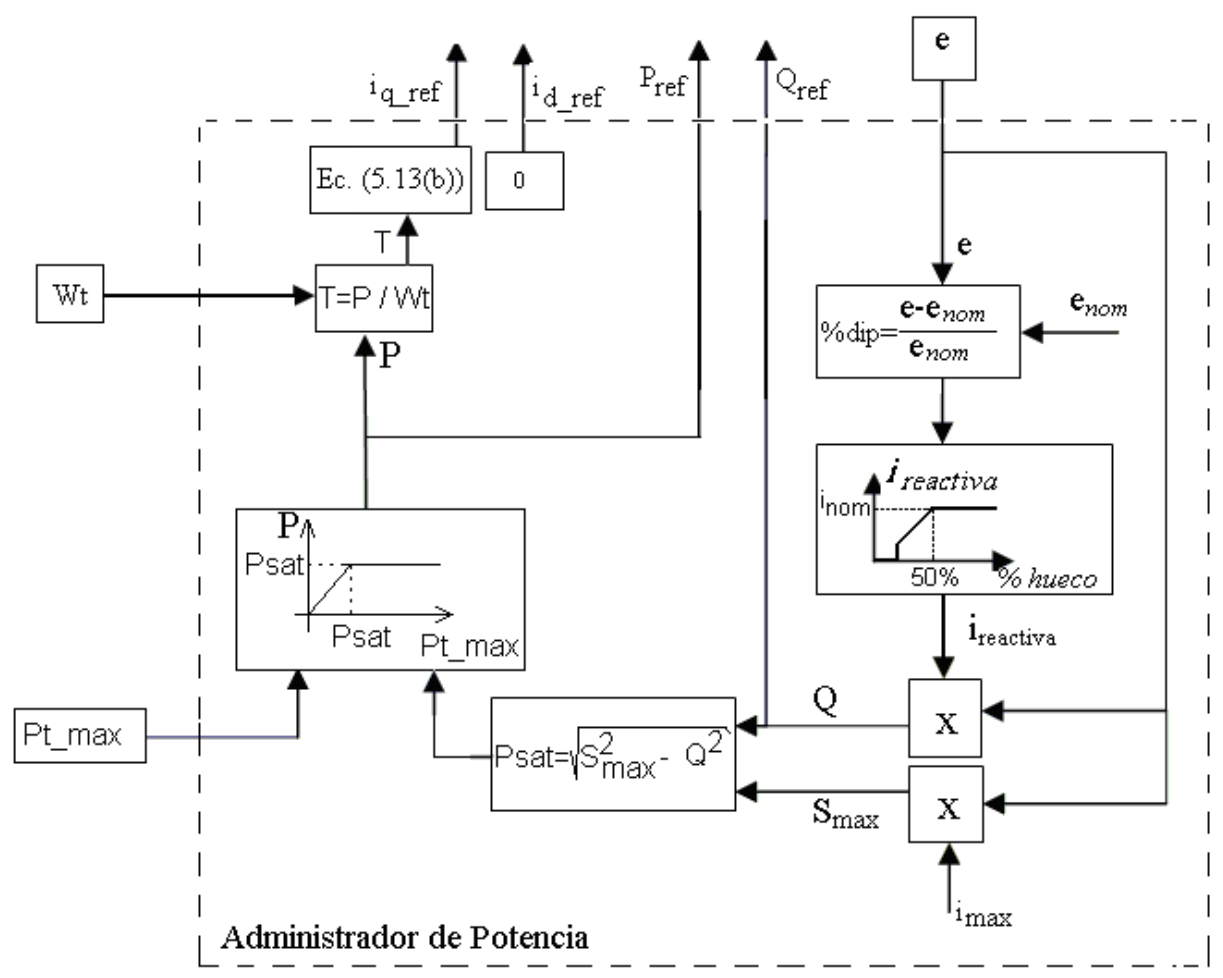

Figura 5.9. Esquema del administrador de potencia.

\subsection{Evaluación de la propuesta}

Las estrategias desarrolladas se aplicaron al convertidor $B 2 B D C M C$ de 5 niveles de la Fig. 5.2 en una turbina de potencia nominal $P_{t_{-} \text {nom }}=3 \mathrm{MW}$ sobre plataforma Simulink. Las pruebas propuestas pretendieron determinar el comportamiento de las variables eléctricas y mecánicas en condiciones normales de funcionamiento y en presencia de fallas en la tensión de la red. Esto último permitió evaluar la capacidad del sistema para soportar las perturbaciones y para cumplir los $G C$. La tensión del bus $D C$ se fijó en $V_{D C}=20 \mathrm{kV}$ y los capacitores individuales en $C=1500 \mu \mathrm{F}$. La inductancia de acoplamiento se estableció en $L_{C}=10 \mathrm{mH}$ y la frecuencia de muestreo y de ejecución de ambos algoritmos predictivos se eligió en $f_{s}=6 \mathrm{kHz}$. Se supuso para la parte mecánica un momento de inercia equivalente $J=10^{6} \mathrm{kgm}^{2}$, valor adoptado de [153] para una máquina de similar potencia. 
Las pruebas realizadas consistieron en verificar el comportamiento del sistema ante un cambio de velocidad de viento para condiciones normales en la red. Por otro lado se analizó el comportamiento del sistema en presencia de un hueco de tensión con amplitud leve y luego en presencia de un hueco con amplitud severa y tiempo de sostenimiento máximo (Fig. 5.7(a), punto "A"). Esta última situación comprendió el caso más exigente del $G C$ y más desfavorable para la turbina en términos de capacidad de frenado sobre el eje de la máquina ya que implicó un tiempo de retención máximo de 1500ms (Fig. 5.7(a)) con capacidad nula de frenado en el eje.

\section{Test en funcionamiento normal. Variación de la velocidad de viento.}

En el principio de la prueba $(t=0)$ la turbina operaba a una velocidad de viento inferior a la nominal con un valor $V_{W}=10 \mathrm{~m} / \mathrm{s}$ con la máquina en estado estacionario. El rotor de la turbina giraba a una velocidad angular $n_{t}=15 R P M$ (Fig. 5.10(a)) y entregaba una potencia de $1,7 M W$. En $t=0,3 \mathrm{~s}$, la velocidad de viento se incrementó súbitamente al valor nominal de la turbina $V_{W}=11,8 \mathrm{~m} / \mathrm{s}$ de forma que la velocidad alcanzó $17,5 R P M$ (Fig. 5.10(a)) con una potencia de salida de 2,9MW. Como puede observarse, el rotor tarda aproximadamente 14 segundos en estabilizarse al valor final de velocidad. El par de frenado que ejerce el generador sobre el eje de la turbina (Fig. 5.10(b)) también se incrementó alcanzando un valor promedio de 1,5MNm. La Fig. 5.11 muestra las tensiones y corrientes del GrC y del GeC para ambos valores de velocidad de viento. Puede verse el corrimiento de fase entre corrientes y tensiones de línea con un valor aproximado de 30 grados, lo que pone de manifiesto el factor de potencia unitario impuesto tanto del lado del generador como en el lado de la red. La Fig. 5.11(a) muestra una corriente y una tensión de línea del GrC, mientras que la Fig. 5.11(b) muestra las mismas variables del lado del $G e C$ para $V_{W}=10 m / s(0<t<0,3 s)$. Ambas tensiones de línea presentan la forma de onda de tensión escalonada sintetizada por el $D C M C$, en tanto que la forma de onda de corriente es casi sinusoidal. Se observa en el caso del $G e C$ (Fig. 5.11(b)) que se utiliza un número reducido de niveles para la síntesis de la tensión de salida. Esto se debe a que la velocidad de viento considerada fue menor a la nominal y también lo fue la tensión inducida en bornes del generador. En cambio, para $V_{W}=11,8 \mathrm{~m} / \mathrm{s}$ y $14 \mathrm{~s}<t<15 \mathrm{~s}$, mientras el $G r C$ utilizó el mismo número de niveles que en el caso anterior (Fig. 5.11(c)) pues la tensión pico de la red permaneció constante, el GeC utilizó más niveles ya que tanto la velocidad del rotor como la tensión inducida se 
han incrementado (Fig. 5.11(d)). Además, ambas corrientes poseen amplitudes superiores ya que la potencia de salida se ha incrementado en más de $1 M W$.
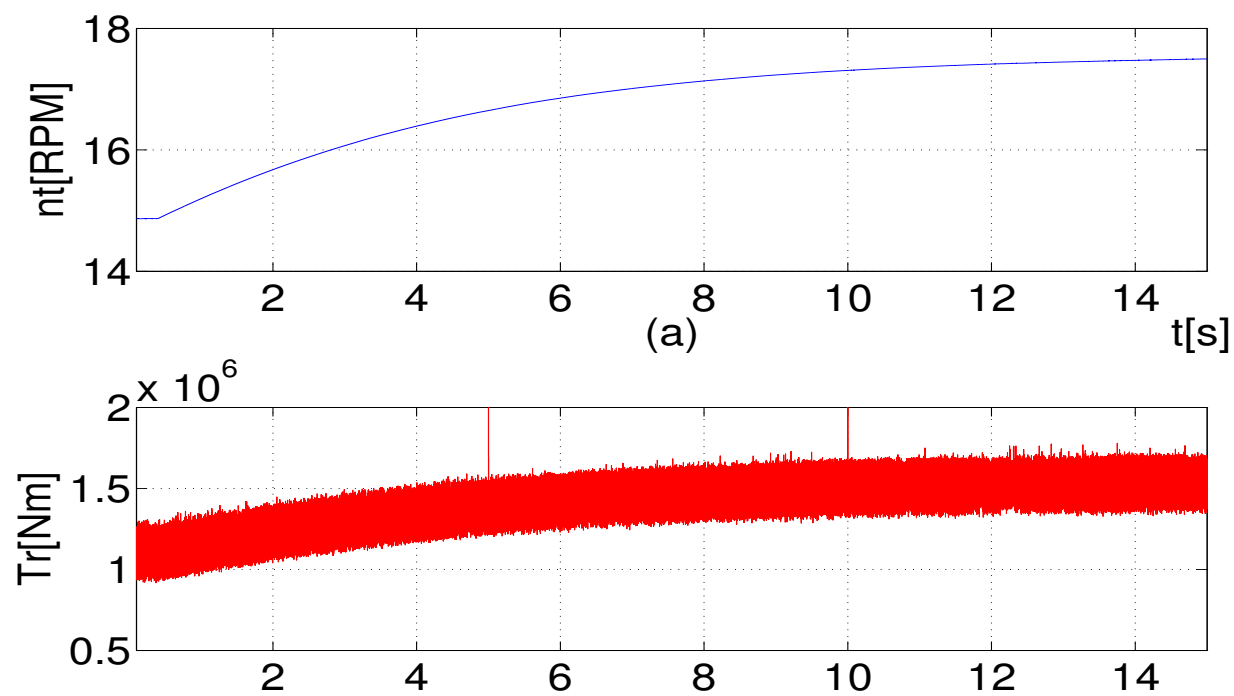

(b) $\mathrm{t}[\mathrm{s}]$

Figura 5.10. (a)Velocidad angular del rotor (b) Par resistente.

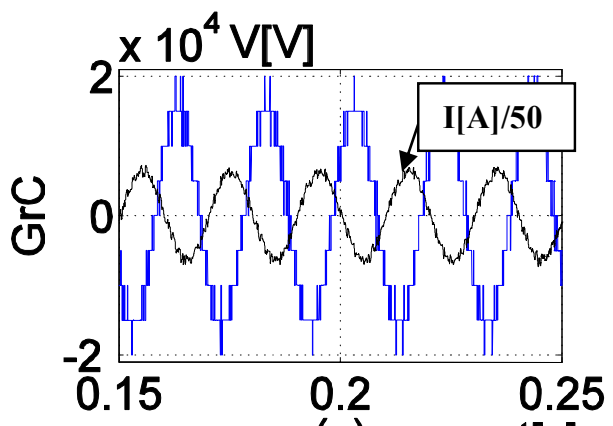

(a)

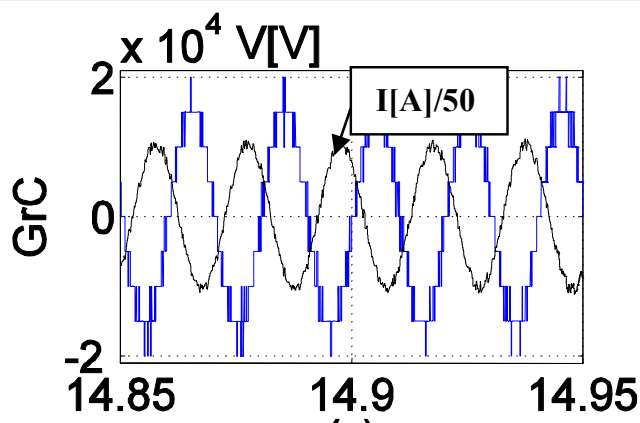

(c)

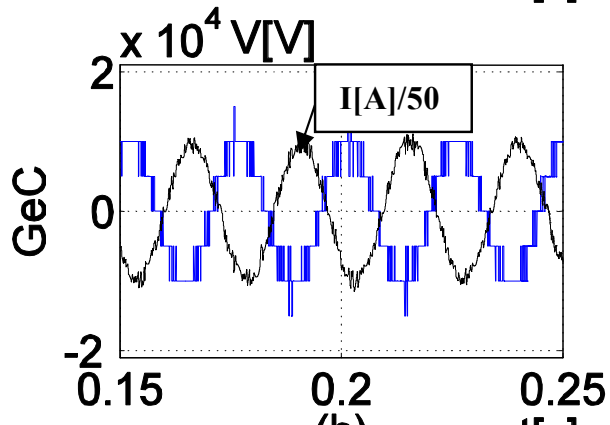

(b) $\mathrm{t}[\mathrm{s}]$

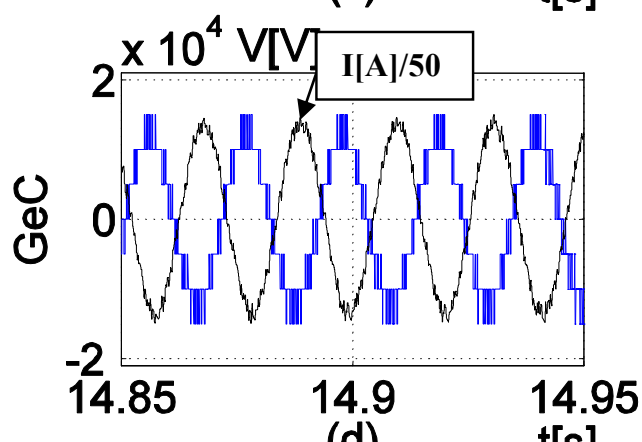

(d)

$\mathrm{t}[\mathrm{s}]$

Figura 5.11. Tensiones y corrientes en bornes del GrC y GeC para dos velocidades de viento diferentes, (a) $G r C, V_{W}=10 \mathrm{~m} / \mathrm{s}$, (b) $G e C, V_{W}=10 \mathrm{~m} / \mathrm{s}$, (c) $G r C$, $V_{W}=11,8 \mathrm{~m} / \mathrm{s}$. (d) $\mathrm{GeC}, V_{W}=11,8 \mathrm{~m} / \mathrm{s}$. 


\section{Hueco de tensión con inyección parcial de potencia reactiva}

El segundo test consistió en aplicar un hueco de tensión de amplitud moderada y verificar la inyección de potencia reactiva a la red. Inicialmente la turbina funcionaba con un viento incidente de $10,8 \mathrm{~m} / \mathrm{s}$ y una potencia aproximada de $2 \mathrm{MW}$. En $t=0,2 \mathrm{~s}$, se introdujo un hueco de tensión con una amplitud del 30\%. De acuerdo con el GC de la Fig. 5.7(b), se debe inyectar una corriente reactiva del $60 \%$ de la corriente nominal para contribuir con el mantenimiento de la tensión. La turbina debe ser capaz de soportar esta condición por 2,2s y restituir la condición previa luego de la falla. La Fig. 5.12 muestra las correspondientes tensiones y corrientes en ambos terminales de alterna. La Fig. 5.12(a) muestra una tensión y una corriente de línea del $G r C$ en las cercanías del instante de falla del sistema, mientras que la Fig. 5.12(b) lo hace para el terminal $A C$ del $G e C$. En el caso del GrC (Fig. 5.12(a)) se observa que el número de niveles con el cual se sintetizan las tensiones de salida se reducen debido al hueco de tensión en la línea. En la Fig. 5.12(b) se manifiesta la rápida reducción de la potencia extraída del generador al mismo tiempo que la potencia procesada por el $G r C$. Esto es importante ya que un pequeño desbalance entre la potencia entregada por el $G e C$ y la drenada por el $G r C$ podría generar una sobretensión en el bus de continua. La Fig. 5.13 muestra una tensión de fase de la red de distribución y su corriente de línea durante el evento. Puede notarse que el cambio en la fase de la corriente desde una corriente puramente activa a otra parcialmente activa y reactiva se efectúa rápidamente. El cociente entre ambas está determinado por la demanda de corriente reactiva por parte del $G C$ y el cupo residual de potencia activa asignado según el esquema de la Fig. 5.8. 


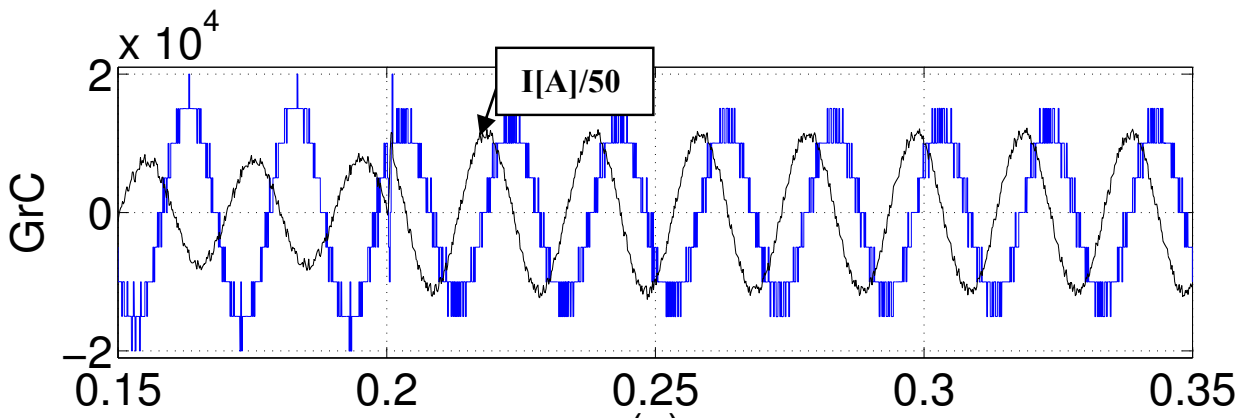

(a)

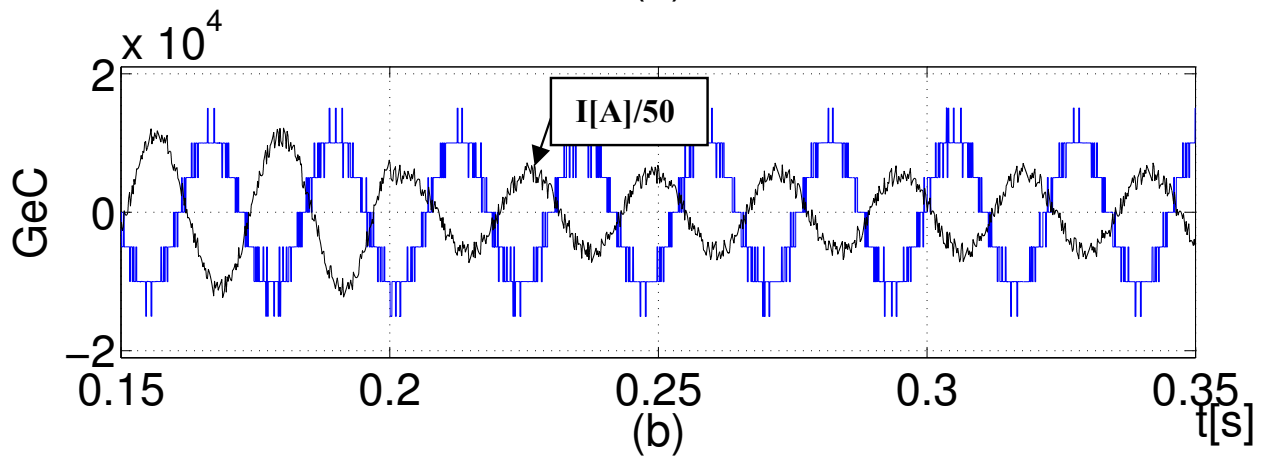

Figura 5.12. Tensión y corriente de línea en ambos convertidores GeC y GrC.

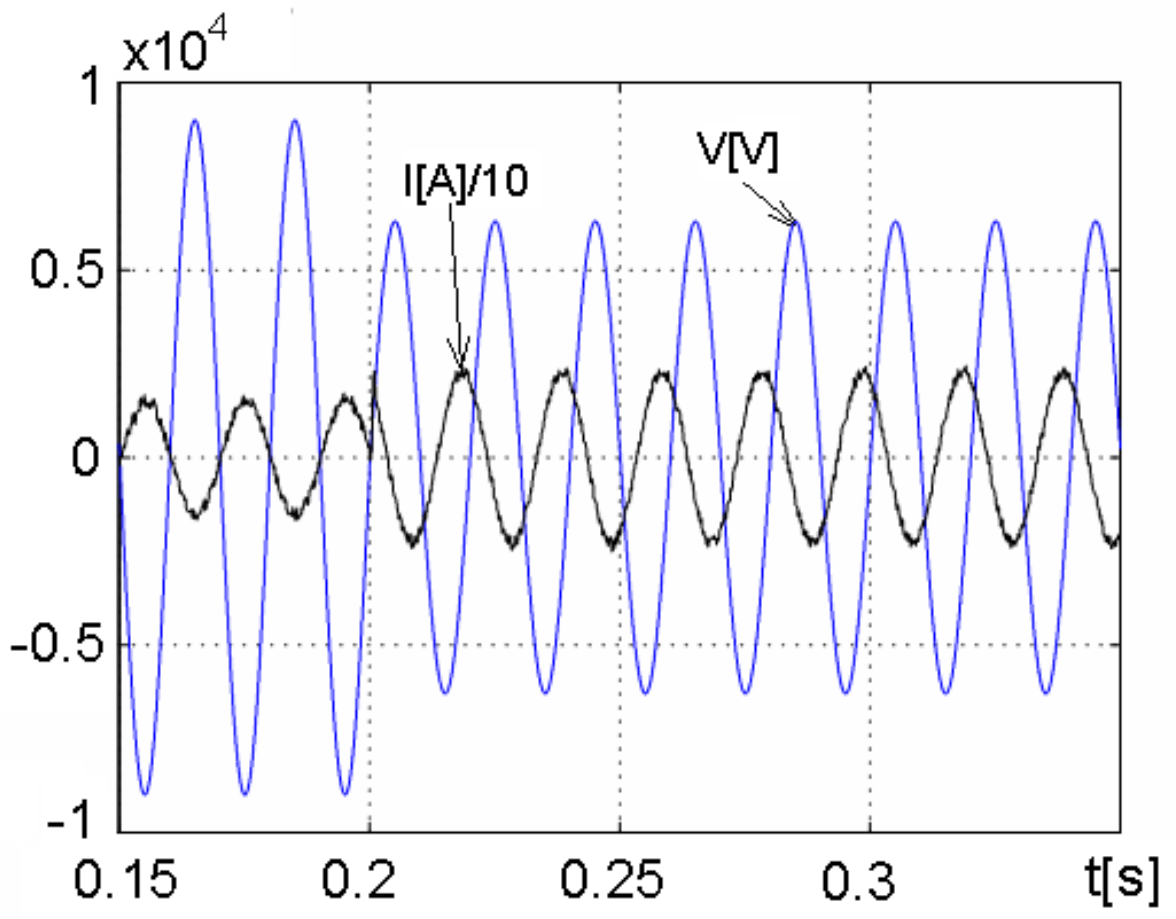

Figura 5.13. Tensión de fase del sistema y corriente de línea.

Las variables mecánicas del generador se muestran en la Fig. 5.14 dentro del lapso de falla. La velocidad de la turbina se muestra en la Fig. 5.14(a) y el par resistente se muestra en la Fig. 5.14(c). Se observa en la primera la aceleración del rotor y en la 
segunda el frenado parcial debido a la capacidad residual para seguir transfiriendo potencia activa. La velocidad del rotor se incrementa desde 16 hasta $17 R P M$ sobre el final de la misma. Entonces, la energía en exceso de la masa rotante es evacuada luego del evento alcanzando la potencia de salida un valor aproximado de $2,5 \mathrm{MW}$ en $t=2,4 \mathrm{~s}$. Las potencias activa y reactiva también se muestran en la Fig. 5.14. Se advierte que durante la falla la potencia activa transferida se reduce de $2 \mathrm{MW}$ a 1,2MW (Fig. 5.14(b)) debido a la reducción parcial de carga sobre el generador cuando se presenta la falla. También aparece en la Fig. 5.14(d) la rápida respuesta dinámica en la potencia reactiva inyectada para contribuir de inmediato al mantenimiento de la tensión de la red.

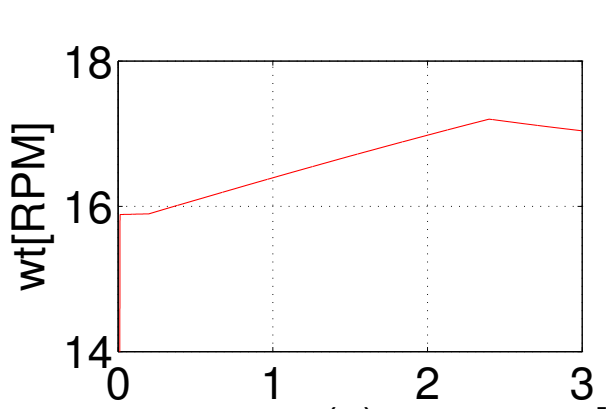

(a)

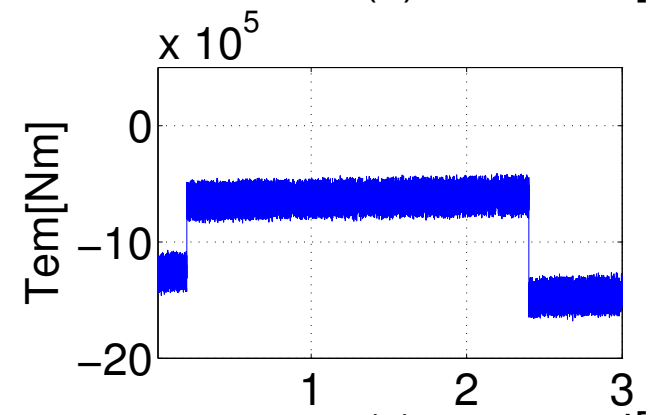

(c)

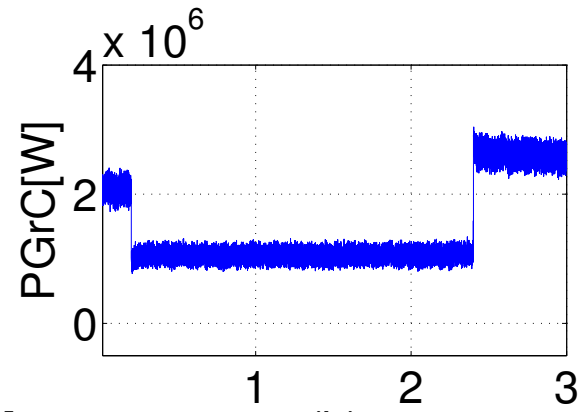

(b)

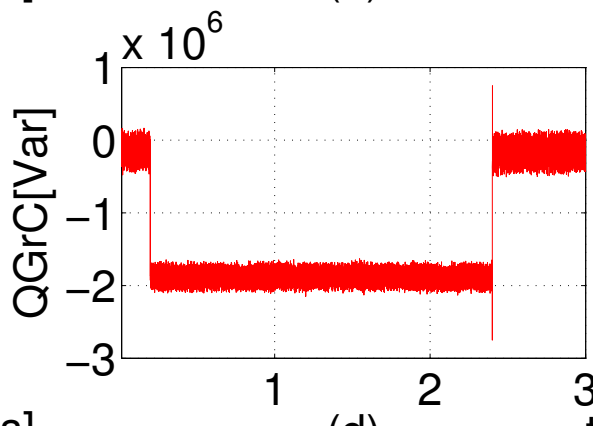

(d)

Figura 5.14. Variables resultantes ante un hueco del 30\% (a) Velocidad de rotación de la turbina, (b) Potencia activa inyectada a la red, (c) Par resistente, (d) Potencia reactiva consumida por el GrC.

\section{Hueco de tensión con inyección total de potencia reactiva. Caso de tiempo de retención máximo}

En este caso, las condiciónes iniciales de velocidad de viento y de potencia de salida fueron $V_{W}=11,8 \mathrm{~m} / \mathrm{s}$ y $P=2,9 \mathrm{MW}$, respectivamente. En $t=0,2 \mathrm{~s}$ se presenta un hueco de tensión del 50\%. De acuerdo con la Fig. 5.7(b), se debe inyectar una corriente reactiva del $100 \%$ de la corriente nominal del convertidor. La turbina debe ser capaz de transitar 
esta condición durante un tiempo de hasta 1,5s (Fig. 5.7(a)) y, luego de la falla, retornar rápidamente a la condición previa.

La Fig. 5.15 muestra las tensiones y las corrientes del convertidor $G e C$ y $G r C$ en ambos lados de alterna. En este caso la Fig. 5.15(b) hace evidente el desacoplamiento total del generador del sistema, quedando en vacío durante la falla. En la Fig. 5.16 la misma tensión de fase que en el caso anterior y su correspondiente corriente de línea. Se observa que el cambio de factor de potencia resulta casi inmediato, aportando rápidamente la potencia reactiva dictada por el $G C$.

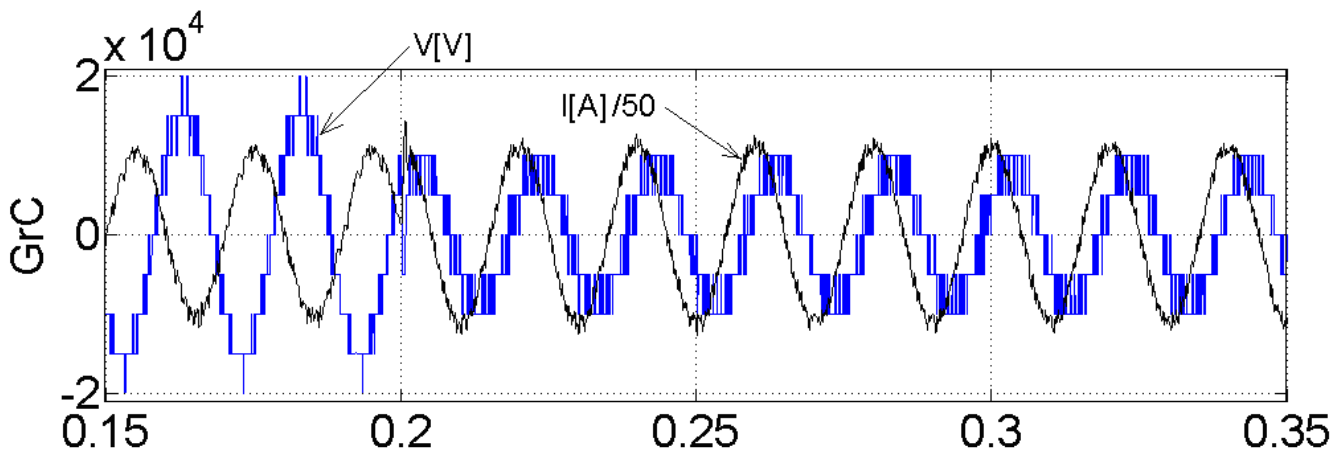

(a)

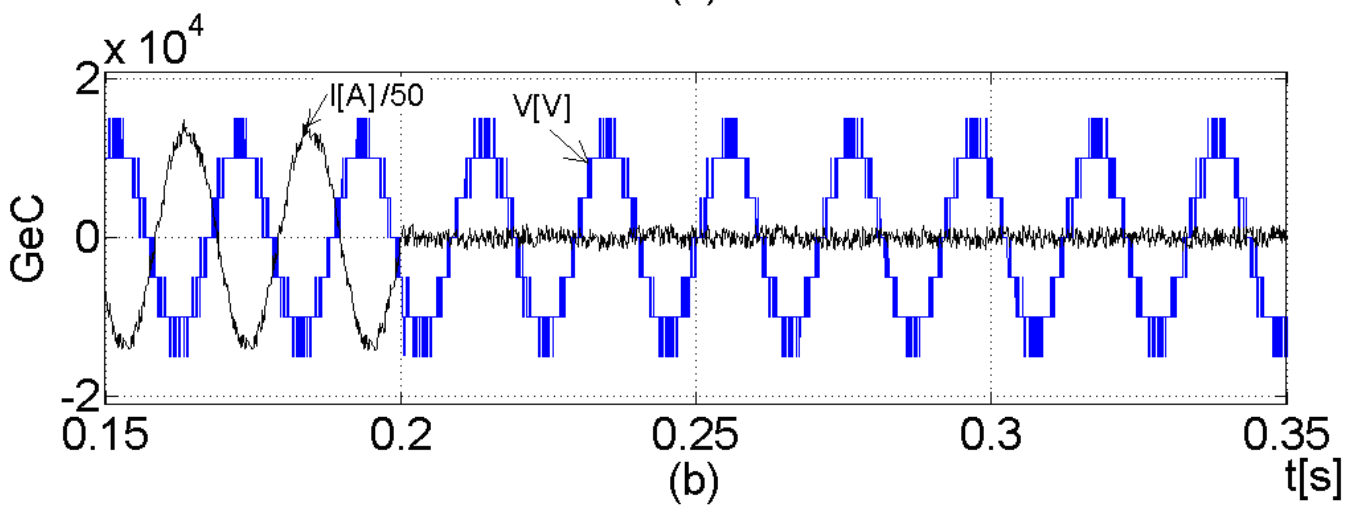

Figura 5.15. Tensiones y corrientes durante el hueco. (a) Tensión y corriente en el $\mathrm{GrC}$, (b) Tensión y corriente en el GeC. 


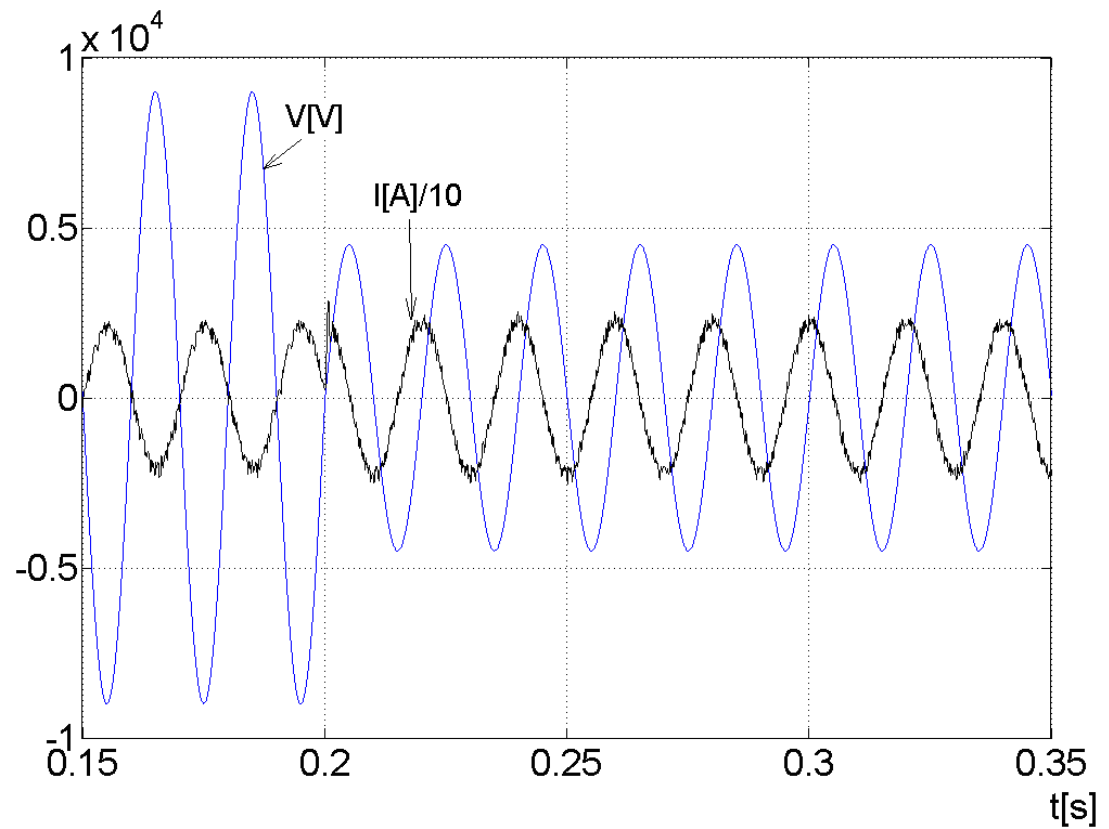

Figura 5.16. Tensión de fase de la red y su correspondiente corriente de línea.

La velocidad de la turbina se muestra en la Fig. 5.17(a) y el par resistente en la Fig. 5.17(c). Se verifica la aceleración del eje debido a la descarga total del generador por parte del $\mathrm{GeC}$ durante el hueco. El incremento de velocidad del rotor se encuentra dentro del $10 \%$ de la velocidad nominal y no resulta significativo debido al alto valor de la inercia del sistema y el corto período de duración del evento, que a su vez es el máximo exigido por el $G C$ (Fig. 5.7). Luego de la falla, la energía almacenada en exceso por la masa rotante es evacuada nuevamente con la limitación de la potencia máxima que puede transferir el $G r C$ a la red. Esto se verifica en la Fig. 5.17(a) por la pendiente negativa de la velocidad de la turbina después de $t=1,7 \mathrm{~s}$. Las potencias activa y reactiva del lado de la red se muestran en las Figs. 5.17(b) y (d), respectivamente. Se observa la anulación de la potencia activa durante la falla debido a que la amplitud del hueco requiere la inyección de corriente puramente reactiva. Se observa también el desacoplamiento total del generador mientras que la inyección de reactivo se inicia rápidamente y se mantiene durante el hueco. Debido a que el hueco posee una amplitud del $50 \%$, la potencia aparente máxima que puede entregar el $\mathrm{GrC}$ en estas condiciones es la mitad de la potencia nominal de la turbina. Esto se verifica en la Fig. 5.17(b) donde la potencia reactiva inyectada asciende a 1,5MVar. 

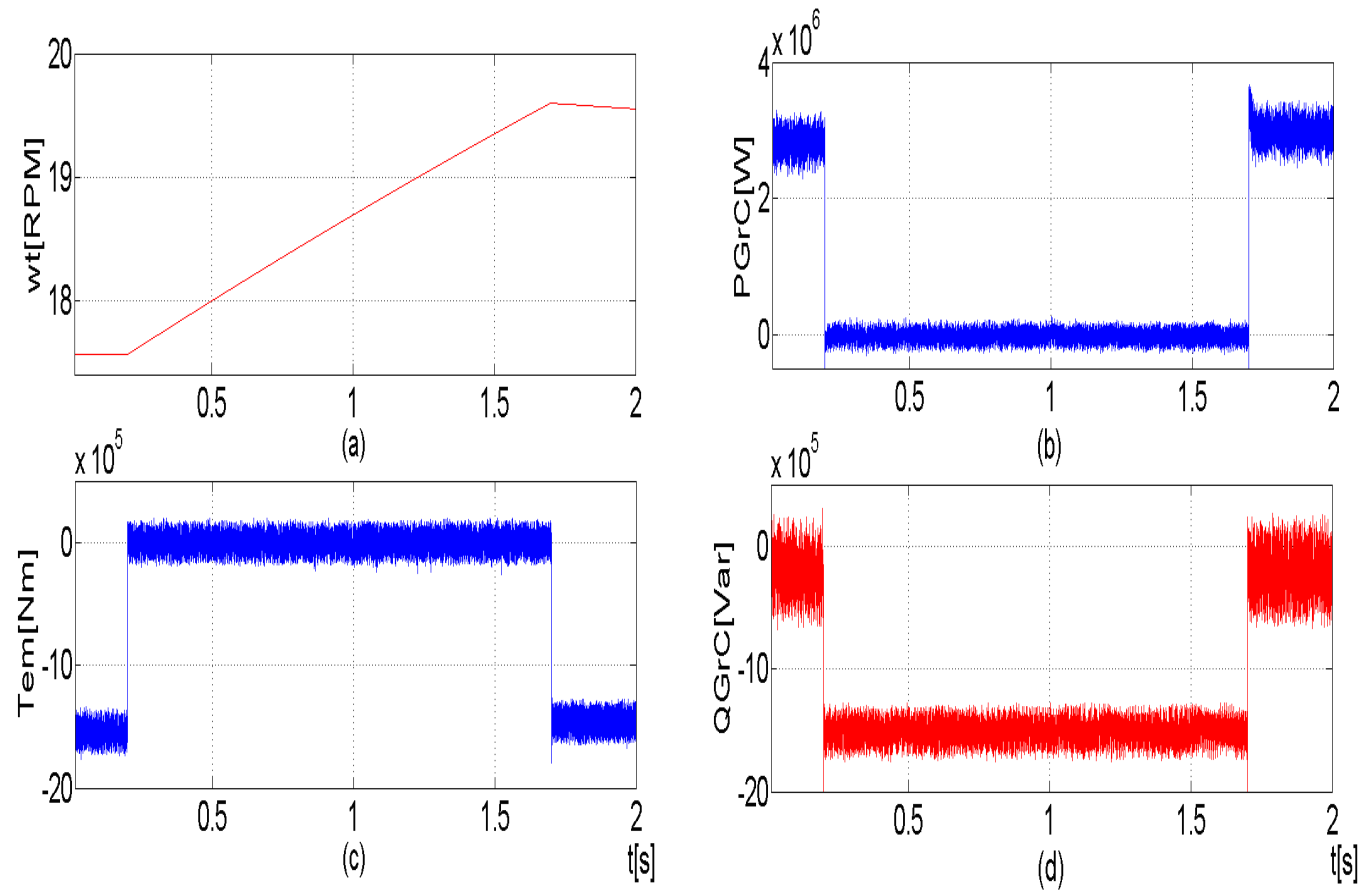

Figura 5.17. Variables resultantes ante un hueco del 50\%(a) Velocidad de rotación de la turbina, (b) Potencia activa inyectada a la red, (c) Par resistente, (d) Potencia reactiva consumida por el GrC.

Finalmente, las tensiones en los capacitores se muestran en la Fig. 5.18(a), y la tensión del bus en la Fig. 5.18(b). Aún en presencia de la severa perturbación en la red, los capacitores permanecen en condición de balance con una desviación menor al $2 \%$, mientras que el valor de la tensión del $D C$ bus sufre un incremento del $1 \%$. En condiciones estacionarias de transferencia de potencia nominal el ripple es $80 V_{p p}$, lo cual representa el 1,6\% del valor de tensión sobre cada capacitor, mientras que durante el hueco se reduce a $50 V_{p p}$. 


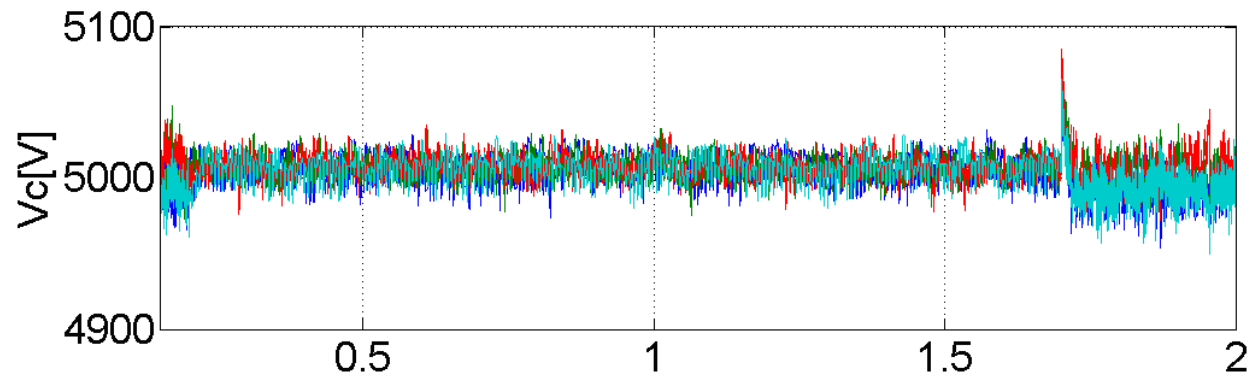

(a)

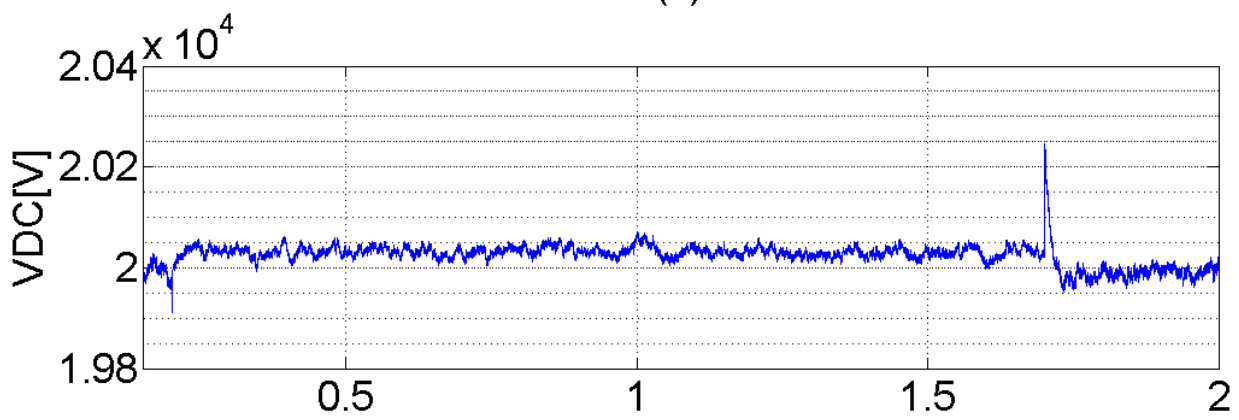

(b)

$\mathrm{t}[\mathrm{s}]$

Figura 5.18. (a) Tensiones en los capacitores, (b) Tensión del bus DC.

\subsection{Conclusiones}

En este capítulo se demostró la capacidad del convertidor DCMC para realizar la interfaz a red de una turbina eólica de gran potencia. Gracias a la capacidad del convertidor $D C M C$ de operar en el rango de media tensión la interfaz de la turbina con la red puede prescindir del transformador de acoplamiento. Los algoritmos propuestos demuestran un comportamiento satisfactorio con respecto al control de las características de operación básicas del sistema y del convertidor, como así también aquellos relacionados con la cumplimentación de los códigos exigidos por el operador de la red. Se puede concluir que la técnica de control predictivo de estados finitos proporciona un esquema apropiado para el control de los convertidores en este tipo de aplicaciones debido a que presenta una dinámica correcta para el cumplimiento de los códigos de red, como así también en lo referente a su facilidad de comprensión e implementación. También, el caso planteado presenta la característica interesante de la autonomía e independencia de ejecución de ambas rutinas de control FCS-MPC, que constituye un aspecto de optimización de relevancia en cuanto al requerimiento de la plataforma de cómputo. 


\section{Capítulo 6}

\section{$\underline{\text { Conclusiones }}$}

\subsection{Conclusión}

Los convertidores multinivel constituyen una tecnología promisoria en aplicaciones de procesamiento de potencia en media tensión. Esto se debe a la capacidad de las topologías para extender la tensión de operación de los convertidores por encima de la tensión de bloqueo admisible de los dispositivos de conmutación y también a la posibilidad de sintetizar tensiones de salida con bajo contenido armónico. Sin embargo los aspectos de control propios de los convertidores son todavía materia de estudio.

En esta tesis se analizó el convertidor multinivel con enclavamiento por diodos y sus aplicaciones de conversión de potencia en el rango de media tensión. El estudio y la caracterización de la topología permitió identificar sus condicionamientos operativos, concluyendo dicho análisis en un conjunto de tópicos básicos a considerar:

- Balance del bus de continua.

- Características del mecanismo de enclavamiento de las tensiones de bloqueo de los dispositivos de conmutación.

- Lógica de conmutación de las columnas del convertidor.

- Condicionamientos en la transición de estados de conmutación.

Este conjunto de variables son internas al convertidor y su consideración en el marco de la estrategia de control constituye un factor crítico en relación a su confiabilidad de operación. El inconveniente principal de la topología, el balance de las tensiones del bus 
de continua, fue atacado utilizando un algoritmo sencillo para la determinación de las secuencias de conmutación óptimas. Esta propuesta no requiere circuitos adicionales de balance y tiene carácter general en términos del número de niveles del convertidor. Esta técnica se combinó con el algoritmo de balance evaluándose su desempeño en un convertidor con frente pasivo. Pudo concluirse que el conjunto presenta limitaciones en cuanto estabilidad del balance del bus de capacitores dependiendo de las condiciones de funcionamiento, hecho coincidente con lo observado en la bibliografía. En una aplicación del convertidor como compensador de potencia reactiva se verificó la acción correctiva del algoritmo en el balance del bus de continua, incluso en presencia de perturbaciones.

También se exploró el control del convertidor mediante la técnica de control predictivo de estados finitos. En base a las consideraciones de conmutación de la topología se sintetizó una regla que domina las transiciones de estados sucesivos y que define el conjunto de control en cada periodo de muestreo. Esto reduce la cantidad de elementos del conjunto a un máximo igual al de un convertidor de tres niveles, independientemente del número de niveles del convertidor considerado, aliviando significativamente la carga computacional. Luego, las variables internas y externas al convertidor son precalculadas a partir de modelos dinámicos para todos los elementos de dicho conjunto. La selección del estado de conmutación del convertidor se realiza en forma directa a partir de funciones de costo, sin la necesidad de moduladores externos. Como aplicación del método se propuso el control de corriente de un convertidor con frente pasivo como filtro de armónicas.

También se analizó el accionamiento de un motor de inducción, y como interfaz de potencia en una turbina eólica, ambos mediante un convertidor DCMC con frente activo (configuración back to back). Utilizando el algoritmo de balance y realizando un análisis del comportamiento de las tensiones del bus de continua en función del nivel y dirección del flujo de potencia, incluso en condiciones de alto factor de potencia e índice de modulación, se concluye y se verifica el balance del bus por el efecto complementario entre ambos convertidores. La estrategia de control elaborada es independiente y autonoma sobre cada convertidor, no requiriendo intercabio mutuo de información acerca del estado de conmutación de cada uno de ellos. En ambos casos se 
arribó a resultados satisfactorios de funcionamiento, pudiendo cumplir con las condiciones operativas impuestas.

Finalmente, vale destacar que la sencillez del algoritmo de control predictivo y su flexibilidad hacen de esta técnica una opción muy interesante para el control de los convertidores de potencia, especialmente cuando es necesario un control multivariable.

\subsection{Trabajos futuros}

El método de control predictivo desarrollado en la tesis conforma, quizá, la alternativa más interesante para seguir explorando. En este sentido, su implementación en otras topologías multinivel, incluso en las topologías híbridas, constituye un camino abierto a una gran variedad de alternativas de trabajo.

Por otra parte, también existe una multiplicidad de aplicaciones de los convertidores multinivel con fuentes de generación alternativas a la eólica, como paneles solares o celdas de combustible. Por ejemplo, los convertidores multinivel pueden utilizarse para sintetizar formas de onda de tensión con bajo contenido armónico mediante la sustitución de los capacitores por fuentes de tensión continua independientes. Estos módulos podrían ser energizados a partir de celdas de combustible o paneles solares. De esta forma sería posible sintetizar tensiones de salida en el nivel de baja tensión (220$380 \mathrm{~V}$ ) a partir de módulos de $48 \mathrm{~V}$. 



\section{Referencias}

[1] A. Gosh y G. Ledwich, Power Quality Enhancement Using Custom Power Devices, Kluwer Academic Publishers, 2002.

[2] N. Hingorani y L. Gyugyi, Understanding FACTS, Concepts and Technology of Flexible AC Transmission Systems, New York: IEEE Press, 2000.

[3] H. Akagi, "Prospects of New Technologies for Power Electronics in the $21^{\text {st }}$ Century", in proc. of the Transmission and Distribution Conference and Exhibition: Asia Pacific, IEEE/PES, pp. 1399-1404, 2002.

[4] B. Ozpineci, M. S. Chintavali y L. M. Tolbert, "Enhancing Power Electronic Devices with Wide Bandgap Semiconductors", International Journal of High Speed Electronics and Systems, Vol. 16, No. 2, pp. 545-556, 2006

[5] H. Schneider, J. Sanchez, J. Achard, "The Diamond for Power Electronic Devices", in proc. of the European Conference on Power Electronics and Applications (EPE'2005), pp. 1-9, 2005.

[6] J. Dixon, L. Morán, J. Rodríguez y R. Domke, "Reactive Power Compensation Technologies: State-of-the-Art Review", Proceedings of the IEEE, Vol. 93, No. 12, pp. 2144-2164, 2005.

[7] M. Emin Meral, A. Teke y M. Tumay, "Overview of an Extended Custom Power Park", in proc. of the $2^{\text {nd }}$ IEEE International Conference on Power and Energy (PECON'2008), pp. 1364-1368, Johor Baharu, Malaysia, 2008.

[8] B. Wu, High-Power Converters and AC Drives, New York: Wiley-IEEE Press, Mar. 2006.

[9] R. A. Hanna y S. W. Randall, "Medium-voltage Adjustable-speed-drive Retrofit of an Existing Eddy-current Clutch Extruder Application", IEEE Transactions on Industry Applications, Vol. 36, No. 6, pp. 1750-1755, 2000.

[10] B. K. Bose, "Neural Network Applications in Power Electronics and Motor Drives - Introduction and Perspective", IEEE Transactions on Industrial Electronics, Vol. 54, pp. 1-20, Feb. 2007.

[11] M. R. Douiri, T. Nasser, A. Essadki y M.Cherkaoui, "Direct Torque Control of Induction Motor Based on Artificial Neural Networks With Estimate And Regulation Speed Using The MRAS and Neural Pi Controller", Journal of Theoretical and Applied Information Technology, Vol. 20, No. 1, pp. 15-21, 2010.

[12] R.Toufouti, S.Meziane y H. Benalla, "Direct Torque Control for Induction Motor Using Fuzzy Logic", International Journal on Automatic Control and System Engineering, Vol. 6, No. 2, pp. 19-26, 2006. 
[13] N. K. Medora y A. Kusko, "Voltage Stress on 8000 HP Induction Motor due to GTO Current Source Inverter- Case Study", in proc. of the 36th Annual Conference on IEEE Industrial Electronics Society (IECON'2010), pp. 24732478, 2010.

[14] ABB Tecnical Guide No. 5, "Bearing Currents in AC Drive Systems" http://www05.abb.com/global/scot/scot201.nsf/veritydisplay/4f1 197aefcf2a91ac1 25744f003ba3be/\$file/factfiletdm 1 bearing $\% 20$ currents revb en lowres.pdf

[15] L. M. Tolbert, T. J. King, B. Ozpineci, J. B. Campbell, G. Muralidharan, D. T. Rizy, A. S. Sabau, H. Zhang, W. Zhang, Y. Xu, H. F. Huq y H. Liu, "Power Electronics for Distributed Energy Systems and Transmission and Distribution Applications", Report prepared by the Oak Ridge National Laboratory for the U.S. Department of Energy, code ORNL/TM-2005/230, 2005.

[16] L. H. Hansen, L. Helle, F. Blaabjerg, E. Ritchie, S. Munk-Nielsen, H. Bindner, P. Sörensen y B. Bak-Jensen, "Conceptual Survey of Generators and Power Electronics for Wind Turbines", report prepared by the Riso National Laboratory, Roskilde, Denmark, code: Riso R-1205(EN), 2001.

[17] Z. Chen, J. Guerrero y F. Blaajberg, "A Review of the State of the Art of Power Electronics for wind Turbines", IEEE Transactions on Power Electronics, Vol. 24 pp. 1859-1875, 2009.

[18] M. Tsili y S. Papathanassiou, "A Review of Grid Code Technical Requirements for Wind Farms", IET Renewable Power Generation, Vol. 3, No. 3, pp. 308-332, 2009.

[19] P. Rodriguez, A. Timbus, R. Teodorescu, M. Liserre y F. Blaabjerg, "Reactive Power Control for Improving Wind Turbine System Behavior Under Grid Faults", IEEE Transactions on Power Electronics, Vol. 24, No. 7, pp. 1798-1801, 2009.

[20] M. Dahlgren, H. Frank, M. Leijon, F. Owman y L. Walfridsson, "Windformer: Energía Eólica a Gran Escala" http://library.abb.com/GLOBAL/SCOT/scot271.nsf/VerityDisplay/FFA0E9492B 54F51AC1256DDD00346FB2/\$File/31-37\%20M153\%20-\%20SPA.pdf

[21] J. M. Carrasco, L. G. Franquelo, J. T. Bialasiewicz, E. Galvan, R. C. P. Guisado, M. M. Prats, J. I. Leon y N. Moreno-Alfonso, "Power-Electronic Systems for the Grid Integration of Renewable Energy Sources: A Survey", IEEE Transactions on Industrial Electronics, Vol. 53, pp. 1002-1016, 2006.

[22] M. Winkelnkemper, F. Wildner y P. Steimer, "Control of a 6MVA Hybrid Converter for a Permanent Magnet Synchronous Generator for Windpower", in proc. of the 18th International Conference on Electrical Machines (ICEM '2008), pp. 1-6., 2008.

[23] S. Kouro, M. Malinowski, K. Gopakumar, J. Pou, L. G. Franquelo, B. Wu, J. Rodriguez, M. A. Perez y J. I. Leon, "Recent Advances and Industrial 
Applications of Multilevel Converters", IEEE Transactions on Industrial Electronics, Vol. 57, pp. 2553-2580, 2010.

[24] H. Abu-Rub, J. Holtz, J. Rodríguez y Ge Baoming, "Medium-Voltage Multilevel Converters - State of the Art, Challenges and Requirements in Industrial Applications", IEEE Transactions on Industrial Electronics, Vol. 57, No. 8, pp. 2581-2596, 2010.

[25] J. Rodríguez, S. Bernet, B. Wu, J. O. Pontt y S. Kouro, "Multilevel VoltageSource-Converter Topologies for Industrial Medium-Voltage Drives", IEEE Transactions on Industrial Electronics, Vol. 54, No. 6, pp. 2930-2945, 2007.

[26] H. Akagi, "Active Harmonics Filters", Proceedings of the IEEE, Vol. 93, No. 12, 2005.

[27] J. Hu, L. Zhang y S. J. Watkings, "Active Power Filtering by a Flying-Capacitor Multilevel Inverter with Capacitor Voltage Balance", in proc. of the International Symposium on Industrial Electronics (ISIE'2008), pp. 2348 - 2352, 2008.

[28] N. Y. Dai, M. Chung Wong y Y. D. Han, "Application of a Three-level NPC Inverter as a Three-Phase Four-Wire Power Quality Compensator by Generalized 3DSVM", IEEE Transactions on Power Electronics, Vol. 21, No. 2, pp. 440-449, 2006.

[29] F. Zare, S. Zabihi y G. Ledwich, "An Adaptive Hysteresis Current Control for a Multilevel Inverter Used in an Active Power Filter", in proc. of the European Conference on Power Electronics and Applications (EPE'2007), pp. 1-8, 2007.

[30] C. Junling, L. Yaohua, W. Ping, Y. Zhizhu y D. Zuyi, "A Closed-Loop Selective Harmonic Compensation with Capacitor Voltage Balancing Control of Cascaded Multilevel Inverter for High-power Active Power Filters", in proc. of the IEEE Power Electronics Specialists Conference, 2008 (PESC'2008), pp. 569-573, 2009.

[31] J. Dionísio Barros y J. F. Silva, "Optimal Predictive Control of Three-Phase NPC Multilevel Converter for Power Quality Applications", IEEE Transactions on Industrial Electronics, Vol. 55, No. 10, pp. 3670-3681, 2008.

[32] H. Akagi, H. Fujita, S. Yonetani y Y. Kondo, "A 6.6-kV Transformerless STATCOM Based on a Five-Level Diode-Clamped PWM Converter: System Design and Experimentation of a 200-V 10-kVA Laboratory Model", IEEE Transactions on Industry Applications, Vol. 44, No. 2, pp. 672-680, 2008.

[33] S. Guanchu , K. Lee , L. Xinchun y L. Chongbo, "New Neutral Point Balancing Strategy for Five-Level Diode Clamped Converters Used in STATCOM of Wind Energy Conversion Systems", in proc. of the 6th International Power Electronics and Motion Control Conference (IPEMC'2009), pp. 2354-2358, 2009.

[34] M. Saeedifard, R. Iravani y J. Pou, "Control and DC-capacitor Voltage Balancing of a Space Vector-modulated Five-level STATCOM", IET Power Electronics, Vol. 2, No. 3, pp. 203-215, 2009. 
[35] Y. Chen, B. Mwinyiwiwa, Z. Wolanski y B. Ooi, "Regulating and Equalizing DC Capacitive Voltages in Multilevel STATCOM", IEEE Transactions on Power Delivery, Vol. 12, No. 2, pp. 901-907, 1997.

[36] Y. Cheng, C. Qian, M. L. Crow, S. Pekarek y S. Atcitty, "A Comparison of Diode-Clamped and Cascaded Multilevel Converters for a STATCOM with Energy Storage", IEEE Transactions on Industrial Electronics, Vol. 53, No. 5, pp. 1512-1521, 2006.

[37] G. Zhu ,G. Wang y D. Hua, "Sliding Mode Control with Variable Structure of Series Active Power Filter", in rec. of the IEEE Power \& Energy Society General Meeting (PES '2009), pp. 1-6, 2009.

[38] S. Kouro, P. Cortés, R. Vargas, U. Ammann y J. Rodríguez, "Model Predictive Control-A Simple and Powerful Method to Control Power Converters", IEEE Transactions on Industrial Electronics, Vol. 56, No. 6, pp. 1826-1838, 2009.

[39] K. Vardar, E. Akpinar y T. Sürgevil, "Evaluation of Reference Current Extraction Methods for DSP Implementation in Active Power Filters", Electric Power Systems Research, Vol. 79, 1342-1352, 2009.

[40] R. Rogrigues Limongi, R. Bojoi, G. Griva y A. Tenconi, "Digital Current Control Schemes", IEEE Industrial Electronics Magazine, Vol. 3, No. 1, pp. 20-31, 2009.

[41] H. Abu-Rub, A. Lewicki, A. Iqbal, J. Guzinski, "Medium voltage drives challenges and requirements", in proc. of the IEEE International Symposium on Industrial Electronics (ISIE'2010), pp. 1372 - 1377, 2010.

[42] "PowerFlex 7000 Medium Voltage Drives with Direct-to-Drive Technology" http://samplecode.rockwellautomation.com/idc/groups/literature/documents/wp/7 000-wp001_en-e.pdf

[43] "Drive Evolution: Medium Voltage" http://www.tmeic.com/Repository/Brochures/Drive_Evolution_2007 hires 12567 49433.pdf

[44] P. Cortés, M. Kazmierkowski, R. Kennel, D. Quevedo y J. Rodríguez, "Predictive Control in Power Electronics and Drives", IEEE Transactions on Industrial Electronics, Vol. 55, No. 12, pp. 4312-4324, 2008.

[45] T. Laczynski y A. Mertens, "Predictive Stator Current Control for Medium Voltage Drives With LC Filters", IEEE Transactions on Power Electronics, Vol. 24, No. 11, pp. 2427-2435, 2009.

[46] A. Bouafia, J. P. Gaubert y F. Krim, "Predictive Direct Power Control of ThreePhase Pulsewidth Modulation (PWM) Rectifier Using Space-Vector Modulation (SVM)", IEEE Transactions on Power Electronics, Vol. 25, No. 1, pp. 228-236, 2010 . 
[47] P. Cortés, J. Rodriguez, P. Antoniewicz y M. Kazmierkowski, "Direct Power Control of an AFE Using Predictive Control", IEEE Transactions on Power Electronics, Vol. 23, pp. 2516 - 2523, 2008.

[48] S. Aurtenechea Larrinaga, M. A. Rodríguez Vidal, E. Oyarbide y J. R. Torrealday Apraiz, "Predictive Control Strategy for DC/AC Converters Based on Direct Power Control", IEEE Transactions on Industrial Electronics, Vol. 54, No. 3, pp. 1261-1271, 2007.

[49] P. Correa, M. Pacas y J. Rodriguez, "Predictive Torque Control for Inverter-Fed Induction Machines", IEEE Transactions on Industrial Electronics, Vol. 54, No. 2, pp. 1073-1079, 2007.

[50] J. S. Lai y F. Z. Peng, "Multilevel Converter-A New Breed of Power Converters", IEEE Transactions on Industry Applications, Vol. 32, No. 3, pp. 509-517, 1996.

[51] H. Ng. Chong, M. A. Parker, L. Ran, P. J. Tavner, J. R. Bumby y E. Spooner, "A Multilevel Modular Converter for a Large, Light Weight Wind Turbine Generator", IEEE Transactions on Power Electronics, Vol. 23, pp. 1062-1074, 2008 .

[52] J. Li, Y. Zhu, Hgyan Xu y Honghua Xu, "CPS-SPWM Flying Capacitor ThreeLevel Back-To-Back Converter Applicative Direct-Drive Wind Power Generator System", in proc. of the International Conference on Sustainable Power Generation and Supply (SUPERGEN '2009), pp. 1-6, 2009.

[53] X. Yuan, Y. Li y J. Chai, "A Transformerless Modular Permanent Magnet Wind Generator System with Minimum Generator Coils", in proc. of the Twenty-Fifth Annual IEEE Applied Power Electronics Conference and Exposition (APEC'2010), pp. 2104-2110, 2010.

[54] R. Melício, V. M. F. Mendes y J. P. S. Catalão, "Power Converter Topologies for Wind Energy Conversion Systems: Integrated Modelling, Control Strategy and Performance Simulation", Renewable Energy, Vol. 35, pp. 2165-2174, 2010.

[55] L. M. Fernandez and C. A. Garcia, F. Jurado, "Operating Capability as a PQ/PV Node of a Direct-drive Wind Turbine Based on a Permanent Magnet Synchronous Generator", Renewable Energy, Vol. 35, pp.1308-1318, 2010.

[56] M. Abbes, J. Belhadj y A. B. Abdelghani Bennani, "Design and Control of a Direct Drive Wind Turbine Equipped with Multilevel Converters", Renewable Energy, Vol. 35, pp. 936-945, 2010.

[57] A. Faulstich, J. K. Steinke y F. Wittwer, "Medium Voltage Converter for Permanent Magnet Wind Power Generators up to $5 \mathrm{MW",} \mathrm{in} \mathrm{proc.} \mathrm{of} \mathrm{the}$ European Conference on Power Electronics and Applications (EPE'2005), pp. 19, 2005. 
[58] F. Kieferndorf, M. Basler, L.A. Serpa, J.H. Fabian, A. Coccia y G.A Scheuer, "A New Medium Voltage Drive System Based on ANPC-5L Technology", in proc. of the IEEE International Conference on Industrial Technology (ICIT'2010), pp. 643-649, 2010.

[59] [Online] "PCS-6000, 6MW Wind Power Converter from ABB" http://www05.abb.com/global/scot/scot232.nsf/veritydisplay/6d33ad8c8f18ab24 c125792e00498a03/\$file/PCS6000 for large Wind Turbines Broshure EN R EV-3BHS351272.pdf

[60] S. A. Verne, S. A. González y M. I. Valla, "An Optimization Algorithm for Capacitor Voltage Balance of N-level Diode Clamped Inverters", in proc. of the 34th Annual International Conference of the IEEE Industrial Electronics Society (IECON'2008), Orlando, FL, USA, pp. 3201-3206, 2008.

[61] S. A. Verne, S. A. González y M. I. Valla, "Modulación Vectorial de Inversores Multinivel de Enclavamiento por Diodos con Balance del Bus de Continua", Revista Iberoamericana de Automática e Informática Industrial (ISSN: 16977912), Vol. 6, No. 2, pp. 69-78, 2009.

[62] S. A. Verne, S. A. González y M. I. Valla, "Modulación Vectorial de Inversores Multinivel de Enclavamiento Por Diodos: (parte 1): Coordenadas Hexagonales", en actas del "XXI Congreso Argentino de Control Automático" (AADECA'2008), Actas en CD- cod. A102, Bs As, Argentina, 1-3 Septiembre de 2008.

[63] S. A. Verne, S. A. González y M. I. Valla, "Modulación Vectorial de Inversores Multinivel de Enclavamiento por Diodos: (parte 2): Balance de Tensiones", en actas del "XXI Congreso Argentino de Control Automático" (AADECA 2008), Actas en CD- cod. A103, Bs As, Argentina, 1-3 Septiembre de 2008.

[64] S. A. Verne y M. I. Valla, "Active Power Filter for Medium Voltage Networks with Predictive Current Control", Electric Power Systems Research (ISSN: 0378-7796), Vol. 80, No. 12, pp. 1543-1551, 2010.

[65] S. A. Verne y M. I. Valla, "Active Power Filter with Predictive Current Control of a Diode Clamped Multilevel Inverter", in proc. of the 35th Annual Conference of the IEEE Industrial Electronics Society (IECON'2009), 3-5 November 2009, Porto Portugal, pp. 3211-3216, 2009.

[66] S. A. Verne y M. I. Valla, "Control Predictivo de Corriente para un Convertidor Multinivel con Diodos de Enclavamiento", en actas de "XIII Reunión de Trabajo en Procesamiento de la Información y Control" (RPIC'2009), Rosario, Santa Fe, 16 al 18 de septiembre de 2009, 2009.

[67] S. A. Verne y M. I. Valla, "Predictive Control of a Back to Back Diode Clamped Multilevel Converter", in proc. of the IEEE International Conference on Industrial Technology (ICIT'2010), 14-17 March, Viña del mar- Valparaiso Chile, pp. 1794-1799, 2010. 
[68] S. A. Verne y M. I. Valla, "Predictive Control of a Back to Back Motor Drive Based on Diode Clamped Multilevel Converters", in proc. of the 36th Annual Conference of the IEEE Industrial Electronics Society (IECON'2010), 7-10 Noviembre, Phoenix, Ar., USA, pp. 2972 - 2977, 2010.

[69] S. A. Verne y M. I. Valla, "Control Predictivo de un Convertidor Bidireccional con Diodos de Enclavamiento", Revista Iberoamericana de Automática e Informática Industrial (ISSN: 1697-7912), Vol. 8, pp. 297-308, October 2011.

[70] S. A. Verne y M. I. Valla, "Control Predictivo de un Convertidor Bidireccional con Enclavamiento por Diodos", en actas del "XXII Congreso Argentino de Control Automático" (AADECA'2010), Actas en CD- cod. AF003794 Bs As, Argentina, 31/8-1/9 de 2010.

[71] S. A. Verne y M. I. Valla, "Direct Connection of WECS System to the MV Grid with Multilevel Converters", Renewable Energy (ISSN: 0960-1481), 2011, en prensa.

[72] S. A. Verne y M. I. Valla, "A WTS Power Conversion Stage With Multilevel Converters Operating in the Medium Voltage Range", International Journal of Hydrogen Energy, artículo aceptado el 13/12/2011.

[73] S. A. Verne y M. I. Valla, "Turbina Eólica Equipada con Convertidor Multinivel como Interfaz Directa de Potencia a Red de Media Tensión", en actas del Cuarto Congreso Nacional - Tercer Congreso Iberoamericano del Hidrógeno y Fuentes Sustentables de Energía (HYFUSEN'2011), Mar del Plata, 6-9 de junio de 2011.

[74] D. O. Neacsu, "Towards an All-Semiconductor Power Converter Solution for the Appliance Market", in proc. of the 36th Annual Conference on IEEE Industrial Electronics Society (IECON'2010), pp. 1671-1676, 2010.

[75] G. Chen, R. Burgos, Z. Liang, F. Lacaux, F. Wang, J.D. van Wyk, W.G. Odendaal y D. Boroyevich, "Reliability-Oriented Design Considerations for High-Power Converter Modules", in proc. of the IEEE $35^{\text {th }}$ Annual Power Electronics Specialists Conference (PESC'2004), pp. 419-425, 2004.

[76] T. Friedli, M. L. Heldwein, F. Giezendanner y J. W. Kolar, "A High Efficiency Indirect Matrix Converter Utilizing RB-IGBTs", in proc. of the IEEE 37th Power Electronics Specialists Conference (PESC '2006), pp. 1-6, 2006.

[77] M. Mohr, Friedrich y W. Fuchs, "Comparison of Three Phase Current Source Inverters and Voltage Source Inverters Linked with DC to DC Boost Converters for Fuel Cell Generation Systems", in proc. of the European Conference on Power Electronics and Applications (EPE'2005), pp. 1-10, 2005.

[78] S. Igarashi, S. Miyashita, H. Wakimoto, H. Nakazawa y Y. Okuma, "Advanced Three-level Converter with Newly Developed 1200V Reverse Blocking IGBTs", in proc. of the 14th European Conference on Power Electronics and Applications (EPE'2011), pp. 1-7, 2011. 
[79] H. Akagi, "The Next-Generation Medium-Voltage Power Conversion Systems", Journal of the Chinese Institute of Engineers, Vol. 30, pp. 1117-1135, 2007.

[80] B. Backlund, M. Rahimo, S. Klaka y J. Siefken, "Topologies, Voltage Ratings and State of the Art High Power Semiconductor Devices for Medium Voltage Wind Energy Conversion", in proc of the IEEE Power Electronics and Machines in Wind Applications (PEMWA'2009), pp. 1-6, 2009.

[81] W. McMurray, "Fast Response Stepped-wave Switching Power Converter Circuit", U.S. Patent 3581212, May 25, 1971.

[82] A. Nabae, I. Takahashi y H. Akagi, "A New Neutral-point-clamped PWM Inverter", IEEE Transactions on Industry Applications, Vol. IA-17, No. 5, pp. $518-523,1981$.

[83] T. Meynard y H. Foch, "Multi-level Choppers for High Voltage Applications", European Power Electronics Journal, Vol. 2, No. 1, pp. 45-50, 1992.

[84] T. Meynard y H. Foch, "Dispositif électronique de conversion d'énergie électrique", French Patent 2679715B1, Jan. 29, 1993.

[85] M. Marchesoni, M. Mazzucchelli y S. Tenconi, "A Non Conventional Power Converter for Plasma Stabilization", in proc. of the Power Electronics Specialists Conference, pp. 122-129, 1988.

[86] W. Liqiao, L Ping, Li Jianlin y Z. Zhongchao, "Study on Shunt Active Power Filter Based on Cascade Multilevel Converters", in proc. of the 35th Annual IEEE Power Electronics Specialists Conference (PESC'2004), Aachen-Germany, pp. 3512-3516, 2004

[87] Bin Wu y J. Pou, "High-Power Converters Topologies, Controls and Applications", Tutorial, IECON, 2006.

[88] X. Yuan y I. Barbi, "Fundamentals of a New Diode Clamping Multilevel Inverter" , IEEE Transactions on Power Electronics, Vol. 15, No. 4, pp. 711-718, 2000.

[89] P. Bartolomeüs, P. Le Moigne y J. B. Mba, "Over-Voltage Problems of DiodeClamped Converters During Switchings", in proc. of the European Power Electronics Conference (EPE'2003), 2003.

[90] G. P. Adams, S. J. Finney, A. M. Massoud y B. W. Williams, "Capacitor Balance Issues of the Diode Clamped Multilevel Inverter Operated in Quasi-two State Mode", IEEE Transactions on Industrial Electronics, Vol. 55, No. 8, pp. 30883099, 2008.

[91] G. P. Adams, S. J. Finney, A. M. Massoud y B. W. Williams, "Two Level Operation of a Diode Clamped Multilevel Inverter", in proc. of the International Simposium on Industrial Electronics (ISIE'2010), pp. 1137-1142, 2010. 
[92] C. Hochgraf, R. Lasseter, D. Divan y T. Lipo, "Comparison of Multilevel Converters for Static VAR Compensation", in proc. of the IEEE Industry Applications Society Annual Meeting, 1994., pp. 921-928, 1994.

[93] M. Fracchia, T. Ghiara, M. Marchesoni y M. Mazzucchelli, "Optimized Modulation Techniques for the Generalized N-level Converter", in proc. of the IEEE Power Electronics Specialists Conference (PESC'1992), Vol. 2, pp.1205$1213,1992$.

[94] J. Rodriguez, P. Correa y L. Moran, "A Vector Control Technique for Medium Voltage Multilevel Inverters," Proceedings of the IEEE Applied Power Electronics Conference, Vol. 1, pp. 173-178, 2001.

[95] D. G. Holmes y T. A. Lipo, Pulse With Modulation for Power Converters, New York:Wiley, 2003.

[96] N. Celanovic y D. Boroyevich, "A Fast Space-Vector Modulation Algorithm for Multilevel Three-Phase Converters", IEEE Transactions on Industry Applications, Vol. 37, No. 2, pp. 637-641, 2001.

[97] Jae-Hyeong Suh, Chang-Ho Choi y Dong-Seok Hyun, "A New Simplified SpaceVector PWM Method for Three-level Inverters", IEEE Transactions on Power Electronics, Vol. 16. No. 4, pp. 545-550, 2001.

[98] R. Joetten y C. Kehl, "A Fast Space Vector Control for a Three Level Voltage Source Inverter", in proc. of the European Power Electronics Conference (EPE'1991), Firenze Italy, Vol. 2, pp. 70-75, 1991.

[99] M. Marchesoni y P. Tenca, "Theoretical and Practical Limits in Multilevel MPC Inverters with Passive Front Ends", in proc. of the European Power Electronics Conference (EPE'2001), Aug. 27-29, Graz, Austria, 2001.

[100] J. Pou, R. Pindado y D. Boroyevich, "Voltage-Balance Limits in Four-Level Diode-Clamped Converters With Passive Front Ends", IEEE Transactions on Industrial Electronics, Vol. 52, No. 1 pp. 190-196, 2005.

[101] S. A. Khajehoddin, A. Bakhshai y P. K. Jain, "A Voltage Balancing Method and its Stability Boundary for Five-Level Diode-Clamped Multilevel Converters", in proc. of the IEEE Power Electronics Specialists Conference (PESC'2007), pp. 2204-2208, 2007.

[102] S. Busquets Monge, J. Bordonau y J. Rocabert, "A Virtual-Vector Pulsewidth Modulation for the Four-Level Diode-Clamped DC-AC Converter", IEEE Transactions on Industrial Electronics, Vol. 23, No. 4, 2008.

[103] S. Busquets-Monge, S. Alepuz, J. Bordonau y J. Peracaula, "Voltage Balancing Control of Diode-clamped Multilevel Converters with Passive Front-ends", IEEE Transactions on Power Electronics, Vol. 23, No. 4, pp. 1751-1758, 2008. 
[104] S. Busquets-Monge, J. Bordonau, y J. Rocabert, "Extension of the Nearest-Three Virtual-Space-Vector PWM to the Four-Level Diode-Clamped DC-AC Converter", in proc. of the Power Electronics Specialists Conference (PESC'2007), pp. 1892-1898, 2007.

[105] T. Meynard y H. Foch, "Multi-Level Conversion: High Voltage Choppers and Voltage-Source Inverters", in proc. of the 23rd IEEE Power Electronics Specialists Conference (PESC '1992), Vol. 1, pp. 397-403, 1992.

[106] C. R. Cutler y B. L. Ramaker, "Dynamic Matrix Control-A Computer Algorithm", In rec. of the AIChE National Meeting, Houston, TX, Apr. 1979.

[107] J. Richalet, A. Rault, J.L. Testud y J. Papon, "Model Predictive Heuristic Control: Applications to Industrial Processes", Automatica, Vol. 14, pp. 413-428, 1978.

[108] M. Morari, "Predicting the Future of Model Predictive Control", in rec. of the AIChE Annual Meeting, Systems and Process Control Centennial Session, Philadelphia, USA, 2008.

[109] E. F. Camacho y C. Bordons, Model Predictive Control, Springer-Verlag, 1999.

[110] A. Linder y R. Kennel, "Model Predictive Control of Electrical Drives", in proc. of the $36^{\text {th }}$ Power Electronics Specialists Conference (PESC'2005), Recife Brazil, pp. $1793-1799,2005$.

[111] J. Rodríguez, J. Pontt, C. Silva, P. Correa, P. Lezana, P. Cortés y U. Ammann, "Predictive Current Control of a Voltage Source Inverter", IEEE Transactions on Industrial Electronics, Vol. 54, No. 1, pp. 495-503, 2007.

[112] E. Fuentes, J. Rodriguez, C. Silva, S. Díaz y D. Quevedo, "Speed Control of a Permanent Magnet Synchronous Motor Using Predictive Current Control", in proc. of the International Power Electronics and Motion Control Conference (IPEMC'2009), pp. 390-395, 2009.

[113] R. Vargas, P. Cortés, U. Ammann, J. Rodríguez y J. Pontt, "Predictive Control of a Three-Phase Neutral-Point-Clamped Inverter", IEEE Transactions on Industrial Electronics, Vol. 54, No. 5, pp. 2697-2705, 2007.

[114] G. S. Perantzakis, F. H. Xepapas, S. A. Papathanassiou y S. N. Manias, "A Predictive Current Control Technique for Three-Level NPC Voltage Source Inverters", in proc. of the Power Electronics Specialists Conference (PESC'2005), Recife, Brazil, 12-16 June 2005, pp. 1241-1246.

[115]F. Zare y G. Ledwich, "A New Predictive Current Control Technique for Multilevel Converters", in proc. of the IEEE Region 10 Conference (TENCON'2006), pp. 1-4, 2006.

[116] E. Silva, B. P. Mc Grath, D. Quevedo y G. Goodwin, "Predictive Control of a Flying Capacitor Converter", in proc. of the 2007 IEEE American Control Conference, pp. 3763-3768, 2007. 
[117] P. Cortés, J. Rodríguez, S. Busquets-Monge y J. Bordonau, "Finite - States Model Predictive control of a Four-Level Diode Clamped Inverter", in proc. of the IEEE 39th Power Electronics Specialists Conference (PESC'2008), Rhodes, Greece, 1519 June 2008, pp. 2203-2208.

[118] D. Quevedo, J. A. De Doná y G. C. Goodwin, "Receding Horizon Linear Quadratic Control With Finite Input Constraint Sets", International Journal of Robust and Nonlinear Control, Vol. 14, No. 4, pp. 355-377, 2004.

[119] D. Q. Mayne, J. B. Rawlings, C. V. Rao y P. O. M. Scokaert, "Constrained Model Predictive Control, Stability and Optimality", Automatica, Vol. 36, pp. 789-814, 2000.

[120] G. C. Goodwin, D. Mayne, K. Chen, C. Coates, G. Mizraeva y D. Quevedo, "Opportunities and Challenges in the Application of Advanced Control to Power Electronics and Drives", IEEE International Conference on Industrial Technology (ICIT'2010), pp. 27-39, 2010.

[121] R. P. Aguilera y D.E.Quevedo, "On stability of Finite Control Set MPC Strategy for Multicell Converters", in proc. of the IEEE International Conference on Industrial Technology (ICIT'2010), pp. 1257-1262, 2010.

[122] P. Cortés, S. Kouro, B. La Rocca, R. Vargas, J. Rodríguez, J. I. León, S. Vazquez y L. G. Franquelo, "Guidelines for Weighting Factors Adjustment in Finite State Model Predictive Control of Power Converters and Drives", in proc. of the International Conference on Industrial Technology (ICIT'2009), pp. 1-7, 2009.

[123] H. Akagi, E. Watanabe y M. Aredes, Instantaneous Power Theory and Applications to Power Conditioning, Wiley-IEEE Press, 2007.

[124] M. Yano, S. Abe y E. Ohno, "History of Power Electronics for Motor Drives in Japan", in rec. of the 2004 IEEE Conference on the History of Electronics, pp. 111, 2004.

[125] V. Stefanovich, "Trends in AC Drive Applications", in proc. of the IEEE International Power \& Energy Conference (IPEC'2005), pp. 72-80, 2005.

[126] T. Thasananutariya y S. Chatratana, "Planning Study of Harmonic Filter for ASDs in Industrial Facilities", Transactions on, Industry Applications, Vol. 45, No. 1, pp. 295-302, 2009.

[127] A. Carlsson, "The Back to Back Converter, Control and Design", tesis doctoral, Publicado por Department of Industrial Electrical Engineering and Automation, Lund Institute of Technology, Sweden, 1998.

[128]B. K. Bose, "Power Electronics and Motor Drives Recent Progress and Perspective", IEEE Transactions on Industrial Electronics, Vol. 56, No. 2, pp. 581-588, 2009. 
[129] G. Zanei, E. Cevenini, H. Ruff y O. Ulibas, "Integrated Systems for UPS: New Solutions in the Power Quality Chain", in proc. of the $29^{\text {th }}$ Telecommunications Energy Conference (INTELEC'2007), pp. 582-586, 2007.

[130] [Online] ABB Tecnical Guide No. 5, "Bearing Currents in AC Drive Systems"

[131] M. Marchesoni y P. Tenca, "Diode-Clamped Multilevel Converters: A Practicable Way to Balance DC-Link Voltages", IEEE Transactions on Industrial Electronics, Vol. 49, No. 4, pp. 752-765, 2002.

[132] Z. Pan, F. Z. Peng, K. Corzine, V. Stefanovic, J. M. Leuthen y S. Gataric, "Voltage Balancing Control of Diode-Clamped Multilevel/Inverter Systems", IEEE Transactions on Industry Applications, Vol. 41, No. 6, pp. 1698-1706, 2005.

[133] Z. Pan y F. Z. Peng, "Harmonics Optimization of the Voltage Balancing Control for Multilevel Converter/Inverter Systems", IEEE Transactions on Power Electronics, Vol. 21, pp. 211-218, 2006.

[134] M. Saeedifard, R. Iravani y J. Pou, "A Space Vector Modulation Strategy for a Back-to-Back Five-Level HVDC Converter System", IEEE Transactions on Industrial Electronics, Vol. 56, No. 2, pp. 452-466, 2009.

[135] T. Ishida, K. Matsuse, K. Sasagawa y L. Huang, "Five-level Double Converters for Induction Motor Drives", IEEE Industry Applications Magazine, Vol. 4, pp. 35-44, 2001.

[136] F. Z. Peng., J. Lai, J. Mc Keever y J. Van Coevering, "A Multilevel VoltageSource Converter System with Balanced DC Voltages", in proc. of the IEEE $26^{\text {th }}$ Power Electronics Specialists Conference (PESC'1995), Vol. 2, pp. 1144-1150, 1995.

[137] M. Marchesoni, M. Mazzucchelli y P. Tenca, "An Optimal Controller for Voltage Balance and Power Losses Reduction in MPC AC/DC/AC Converters", in proc. of the IEEE Power Electronics Specialists Conference (PESC'2000), 18-23 June 2000, Galway, Ireland, Vol. 2, pp. 662-667, 2000.

[138] T. Ishida, K. Matsuse, T. Miyamoto, K. Sasagawa y L. Huang, "Fundamental Characteristics of Five-Level Double Converters With Adjustable DC Voltages for Induction Motor Drives", IEEE Transactions on Industrial Electronics, Vol. 49, No. 4, pp. 775-782, 2002.

[139] I. Takahashi y T. Noguchi, "A New Quick-response and High-efficiency Strategy for an Induction Motor", IEEE Transactions on Industry Applications, Vol. 22, No. 7, pp. 820-827, 1986.

[140] M. Depenbrock, "Direct Self Control (DSC) of Inverter Fed Induction Machine", IEEE Transactions on Power Electronics, Vol. 3, No. 4, pp. 420-429, 1988. 
[141] S. Kouro, R. Bernal, C. Silva, J. Rodriguez y J. Pontt, "High Performance Torque and Flux Control for Multilevel Inverter Fed Induction Motors", in proc. of the $32^{\text {th }}$ International Conference of the IEEE Industrial Electronics Society (IECON'2006), Paris, France, pp. 805-810, 2006.

[142] T. Geyer, G. Papafotiou y M. Morari, "Model Predictive Direct Torque Control. Part I: Concept, Algorithm and Analysis", IEEE Transactions on Industrial Electronics, Vol. 56, No. 6, pp. 1894-1905, 2009.

[143] G. Papafotiou, J. Kley, Papadopolus, P. Bohren y M. Morari, "Model Predictive Direct Torque Control. Part II: Implementation and Experimental Evaluation", IEEE Transactions on Industrial Electronics, Vol. 56, No. 6, pp. 1906-1915, 2009.

[144] J. Rodríguez, J. Pontt, P. Correa, P. Lezama y P. Cortés, "Predictive Power Control of an $\mathrm{AC} / \mathrm{DC} / \mathrm{AC}$ Converter", in proc. of the Industry Applications Conference (IAS), Hong Kong (China), Vol. 2, pp. 934 - 939, 2005.

[145] J. C. Ramirez Martinez, R. Kennel y T. Geyer, "Model Predictive Direct Current Control", in proc. of the IEEE International Conference on Industrial Technology (ICIT'2010), pp. 1788-1793, 2010.

[146] D. Quevedo, R. P. Aguilera, M. A. Pérez y P. Cortés. "Finite Control Set MPC of an Active Rectifier with Dynamic References", in proc. of the IEEE International Conference on Industrial Technology (ICIT'2010), 14-17 March, Viña del marValparaiso Chile, pp. 1245-1250, 2010.

[147] "E-126 7.5MW turbine", ENERCON Magazine for Wind Energy, No. 4, 2007.(http://www.enercon.de)

[148] R. Teodorescu, M. Liserre y P. Rodriguez, Grid Converters for Photovoltaic and Wind Power Systems, IEEE-Wiley, 2011.

[149] H.T. Ma y B.H. Chowdhury, "Working Towards Frequency Regulation with Wind Plants: Combined Control Approaches", IET Renewable Power Generation, Vol. 4, No. 4, pp. 308-316, 2010.

[150] H. Bevrani, A. Ghosh y G. Ledwich, "Renewable Energy Sources and Frequency Regulation: Survey and New Perspectives", IET Renewable Power Generation, Vol. 4, No. 5, pp. 438-457, 2010.

[151]E. Muljadi y C. P. Butterfield, "Pitch-controlled Wind Turbines", IEEE Transactions on Industry Applications, Vol. 37, No. 1, pp. 240-246, 2001.

[152] S. Alepuz, S. Busquets-Monge, J. Bordonau, P. Cortés y S. Kouro, "Control Methods for Low Voltage Ride-through Compliance in Grid-connected NPC Converter Based Wind Power Systems Using Predictive Control", Energy Conversion Congress and Exposition (ECCE'2009), pp. 363-369, 2009. 
[153] Joon-Young Park, Jae-Kyung Lee, Ki-Yong Oh, Jun-Shin Lee y Beom-Joo Kim, "Design of Simulator for 3MW Wind Turbine and its Condition Monitoring System", International MultiConference of Engineers and Computer Scientists (IMECS'2010), 2010. 